ESTIMAÇÃO E PRECISÃO NO MODELO DE

REGRESSAO LINEAR COM ERROS NAS

VARIÁVEIS E MENSURACÕES REPLICADAS.

Julio Hokama

TESE APRESENTADA

AO

INSTITUTO DE MATEMÁTICA E ESTATÍSTICA

DA

UNIVERSIDADE DE SÃO PAULO

PARA

OBTENÇÃO DO GRAU

DE

DOUTOR EM ESTATÍSTICA

Área de Concentração: Estatística

Orientador: Prof. Dr. Pedro A. Morettin

Co-Orientador: Prof. Dr. Heleno Bolfarine

Durante a elaboração deste trabalho o autor recebeu Apoio financeiro da CAPES

-São Paulo, julho de 2001- 


\title{
ESTIMAÇÃo E PRECISÃO NO MODELO DE REGRESSÃO LINEAR COM ERROS NAS VARIÁVEIS E MENSURAÇÕES REPLICADAS.
}

\author{
Este exemplar corresponde à redação \\ final da tese de doutoramento, \\ devidamente corrigida e defendida \\ por Julio Hokama e aprovada \\ pela comissão julgadora.
}

São Paulo, 06 de setembro de 2001.

Banca Examinadora:

- Prof. Dr. Pedro A. Morettin (Presidente) - IME/USP

- Prof. Dr. Gilberto Alvarenga Paula Ferrari - IME/USP

- Prof. Dr. Enrico Colosimo - UFMG

- Prof. Dr. Gauss Moutinho Cordeiro - UFBA

- Prof. Dr. Manuel Jesus Galea Rojas - UNIV. VALPARAISO 


\title{
AGRADECIMENTOS
}

\author{
Agradeço
}

às universidades Federal do RJ (UFRRJ) e de São Paulo (USP),

aos professores Dr. Pedro A. Morettin e Dr. Heleno Bolfarine, pelas orientações confiantes,

aos amigos novos e antigos Celso, Deborah, Katia, Mario e Sebastião (a turma de 96); Domingo(s), Sandra, Jairo, Gladys, Hernan, Maurício, Leonardo e Paola (camaradas colombianos); Hélinton, Regina, Luis Gustavo, Delhi, Laura, Sergio e Nelson; por nossas conversas, estímulos no dia-a-dia e as sextas-feiras santas da primeira semana. $\mathrm{E}$ a todos que, direta e indiretamente, contribuíram para tornar este projeto de doutoramento possível.

Deixo, aqui, uma "reflexão " adaptada de uma interpretação livre do pensamento de Gaston Bachelard: "O ser humano já nasce "velho", pois nasce com a idade dos seus pré-conceitos; só o (novo) saber rejuvenesce. É preciso, portanto. manter sempre a postura de aluno para não caducar". 
À Anne, companheira de todas as jornadas. Aos meus pais Taro e Haruko (In memorian) e aos meus irmãos, os outros Hokamas. 


\section{RESUMO}

Nesta tese apresentamos procedimentos baseados em replicações para o problema da falta de identificação em modelos com erros nas variáveis. Discutimos procedimentos para os casos estrutural e funcional. Os procedimentos considerados são baseados nos métodos de momentos e de máxima verossimilhança. Os modelos considerados apresentam extensões para resultados publicados na literatura. A utilização do método de máxima verossimilhança no caso funcional não está na literatura.

\section{ABSTRACT}

In this thesis we present results based on replicated observations for the identificability problem in measurement errors models. We consider procedures for the structural and functional situations. Such procedures are based on maximum likelihood and method of moments approaches. The models considered present extensions over published results. The development of the maximum likelihood situation for the functional case is not in the literature. 


\section{Conteúdo}

1 CONSIDERAÇÕES INICIAIS 1

1.1 Introdução . . . . . . . . . . . . . . . . . 1

1.2 Notações Gerais . . . . . . . . . . . . . . . . . . . . . 1

1.3 Formulação do problema . . . . . . . . . . . . . . . . . 2

1.3 .1 Introdução . . . . . . . . . . . . . . . . 2

1.3.2 Formalização e Notações do Modelo Estrutural Normal . . . . . . . . . . . . . . . . . . 4

1.3.3 Formalização do Modelo Funcional Normal . . . . . . . . . . . 8

1.4 Objetivos da tese e sua organização. . . . . . . . . . . . . . . 9

2 Modelo Estrutural com Replicações na Variável Explanatória $\quad 12$

2.1 Introdução . . . . . . . . . . . . . . . . . . . . . . . 12

2.2 Replicações apenas da variável explanatória . . . . . . . . . . . . . 13

2.2 .1 Log-verossimilhança . . . . . . . . . . . . . . . . 14

2.2.2 Estimadores de Máxima Verossimilhança . . . . . . . . . . 16

2.2 .3 Precisão dos EMV's . . . . . . . . . . . . . . 20

2.3 Replicações não-Balanceadas da Variável Explanatória . . . . . . . . 22

2.3.1 Log-verossimilhança . . . . . . . . . . . . . . . . . . . . 22

2.3.2 Equações de verossimilhança do modelo . . . . . . . . . . . 23

2.3.3 Estimação de Máxima Verossimilhança Usando o Algoritmo EM 24

2.4 Extensão do caso Chan e Mak (1979a): $r_{t}=r$ e $s_{t}=s . \ldots . . . .29$

2.4 .1 Notações e modelo . . . . . . . . . . . . . . . . . . . 29 
2.4 .2 Log-verossimilhança . . . . . . . . . . . . . . . . . . 29

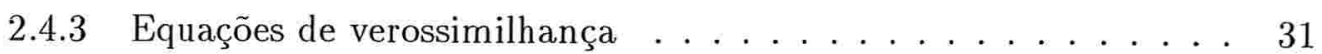

2.4.4 Casos particulares ... . . . . . . . . . . . . 34

2.4.5 Matriz de Covariâncias Assintóticas dos EMV's . . . . . . . . . 35

3 Estimadores Alternativos Envolvendo Replicações da Variável Explanatória

3.1 Introdução . . . . . . . . . . . . . . . . . . . . . . . 39

3.2 Replicações Balanceadas . . . . . . . . . . . . . . . . . . . . . . . 41

3.2.1 Estimador de $\beta$ usando um EMV de $\sigma_{u u} \ldots \ldots \ldots . \ldots 41$

3.2.2 Estimadores de $\beta$ baseados em EMV de $\lambda$ e $k_{x x} \ldots \ldots . .46$

3.3 Replicações Não-Balanceadas . . . . . . . . . . . . . . . . . . . . . . 49

3.3 .1 Introdução . . . . . . . . . . . . . . . . . 49

3.3.2 Estimadores de $\beta$ considerando $S_{u u 1 n} \ldots \ldots \ldots 50$

3.3.3 Estimador de $\beta$ considerando o estimador $S_{u u 2 n} \ldots \ldots \ldots 1$

3.4 Estimadores de $\beta$ corrigidos e ponderados . . . . . . . . . . 66

3.4 .1 Estimador $\hat{\beta}_{N B G}$ corrigido . . . . . . . . . . 68

3.4 .2 Estimadores ponderados melhorados . . . . . . . . . . 75

3.5 Avaliações Numéricas . . . . . . . . . . . . . . . . . . . 86

4 Relação Linear Funcional com Replicações 94

4.1 Introdução . . . . . . . . . . . . . . . . . . . . . . . . . . . . . . . . . . . 94

4.2 Notações e Resultados Preliminares . . . . . . . . . . . . . . . . . . 97

4.3 Aplicação dos Resultados Anteriores ao Modelo Normal Bivariado com Replicações. . . . . . . . . . . . . . . . . . . . . . . . . . . . 98

4.3.1 A função de verossimilhança . . . . . . . . . . . . . . 98

4.4 Cálculos dos EMVs de $\left(\alpha^{(o)}, \beta^{(o)}, \sigma_{u u}^{(o)}, \sigma_{e e}^{(o)}\right)^{\prime} \ldots \ldots \ldots \ldots 10 . \ldots \ldots$

4.4 .1 Casos Particulares . . . . . . . . . . . . . . . . . . . 111

4.5 Matriz de Covariância Assintótica dos EMVs de $\theta^{0}$. . . . . . . . . . 113

4.5 .1 Cálculo da Matriz $E\left[A_{n}\left(\theta^{1}\right)\right] \ldots \ldots \ldots . \ldots \ldots 113$

4.5 .2 Cálculo de $V_{n} \ldots \ldots \ldots \ldots \ldots \ldots$ 
4.6 Avaliações Numéricas . . . . . . . . . . . . . . . . . . . . . . . . . . . . 124

5 Contribuições, Conclusão e Estudos Futuros da Tese 132

5.1 Contribuições . . . . . . . . . . . . . . . . . . . . . . . . . . 132

5.2 Conclusão e Estudos Futuros. . . . . . . . . . . . . . . . . . . . . 133

A Implementação dos Algoritmos EM e do Tipo EM

A.1 Cálculos para Implementação do Algoritmo EM (Schafer e Purdy, 1996) 137

A.2 Cálculos para Implementação do Algoritmo Tipo EM (Kimura, 1992) . . 140

B Teoremas, Lemas e Outros Resultados Básicos 143

B.1 Alguns Teoremas Sobre Matrizes . . . . . . . . . . . . . . . . . . . . 143

B.2 Teoremas e Resultados Assintóticos Utilizados no

Capítulo 3 . . . . . . . . . . . . . . . . . . . . . . . . . . . 144

B.2.1 Referências de teoremas . . . . . . . . . . . . . . . . . . . . 144

B.2.2 Lema de Gleser-Ruppert ... . . . . . . . . . . . . . . . . 145

B.3 Condições de Regularidade de Mak (1982) . . . . . . . . . . . . . 145

C Condições do TCL de Liapounov e do Gleser- Ruppert 149

C.1 Cálculo de $E\left(R_{3 t}\right)^{3}$ e $E\left(R_{3 t}\right)^{4} \ldots \ldots \ldots \ldots$. . . . . . . . 149

C.1.1 Cálculos preliminares . . . . . . . . . . . . . . . 150

C.1.2 Cálculo de $\mathbf{E}\left(\mathbf{R}_{3 \mathrm{t}}^{4}\right) \ldots \ldots \ldots \ldots \ldots \ldots$

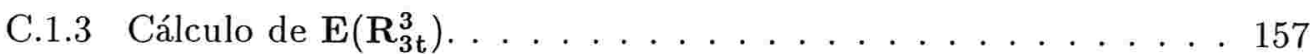

C.2 Obtenção de cotas superiores de $E\left(R_{33 t}^{i}\right)$;

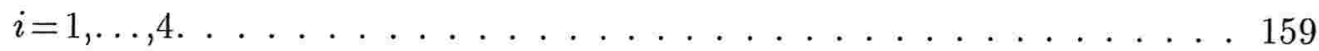

C.3 Prova que $E\left\{\left(U_{i t}\right)^{4}\right\}<\infty, i=1,2,3 \ldots \ldots \ldots \ldots \ldots$

C.3.1 Cálculos preliminares . . . . . . . . . . . . . . 167

C.4 Prova que $\mathbf{E}\left\{\left|\mathrm{U}_{\mathrm{it}}\right|^{3}\right\}<\infty, i=1,2,3 \ldots \ldots \ldots \ldots \ldots$. . . . . . . 172

C.5 Cálculo de $\operatorname{Var}\left(U_{i t}\right), i=1,2,3 \ldots \ldots \ldots \ldots \ldots$. . . . . . . . . . . . . .

D Condições suficientes para que a matriz $E\left[\mathrm{~A}_{n}\left(\theta^{(1)}\right) \mid \theta^{(0)}, x_{t}^{0}\right]$ seja negativa definida 
D.0.1 Condições suficientes para que a matriz $\mathrm{E}_{3}$ tenha determinante negativo . . . . . . . . . . . . . . . 185

D.0.2 Condições suficientes para que a matriz $\mathbf{E}_{4}$ tenha determinante positivo . . . . . . . . . . . . . 186

D.0.3 Casos particulares . . . . . . . . . . . . . . 188 


\section{Capítulo 1}

\section{CONSIDERAÇÕES INICIAIS}

\subsection{Introdução}

Como é razoável esperar de uma introdução de uma tese, apresentamos, neste capítulo, uma exposição geral e articulada das idéias básicas nela discutidas. No entanto, com o intuito de facilitar a comunicação, apresentamos, na seção seguinte, as notações de uso mais geral. Nas outras seções, aparecem nesta ordem: a formulação do problema (modelos estruturais e funcionais normais); os objetivos, a organização e as contribuições da tese; as conclusões e perspectivas de trabalho futuro.

\subsection{Notações Gerais}

Apresentamos, a seguir, as notações gerais mais usadas no decorrer deste texto; as notações mais específicas serão fixadas nos lugares onde delas fizermos uso.

- $\sim N I I D(\mathrm{~m}, \Sigma): \quad$ abreviação para vetores aleatórios normais, independentes e identicamente distribuídos, com vetor de médias $\mathrm{m}$ e matriz de covariâncias $\Sigma$.

- $\operatorname{diag}\left(\sigma_{x x}, \sigma_{u u}, \sigma_{e e}\right)$ denota uma matriz diagonal com os elementos dados na diagonal.

- $\hat{\theta}$ Estimador de Máxima Verossimilhança (EMV) do vetor paramétrico 
- $\mathrm{A}^{\prime}$ : transposta da matriz A.

- $\mathbf{A}^{-1}$ : inversa da matriz $\mathbf{A}$.

- iid $(.,$.$) : vetores aleatórios independentes e identicamente distribuídos com$ o vetor de médias e matriz de covariâncias dados entre parênteses.

- $1_{r s}$ : é uma matriz de dimensão $r \times s$ com todos os elementos iguais a 1 .

- $0_{r s}$ : é uma matriz de dimensão $r \times s$ cujos elementos são todos iguais a zero.

- $I_{r}$ : é a matriz identidade de ordem r.

- $[\mu]: \quad \mu$-quase certamente.

- $\|A\|: \quad$ é o determinante de A.

\subsection{Formulação do problema}

\subsubsection{Introdução}

Neste trabalho, consideramos o Modelo de Regressão Linear Simples com erros nas variáveis, que na literatura estatística refere-se ao modelo descrito pela relação linear

$$
y_{t}=\alpha+\beta x_{t},
$$

em que $y_{t}$ é a variável resposta e $x_{t}$ a variável explanatória (ou preditor, ou covariável), $\alpha$ e $\beta$ são parâmetros desconhecidos, que devem ser estimados. Admitimos, ainda, que as verdadeiras variáveis $\left(x_{t}, y_{t}\right)$ não são observadas diretamente, sendo $\left(X_{t}, Y_{t}\right)$ as variáveis realmente observáveis. Estas variáveis são as verdadeiras, $\left(x_{t}, y_{t}\right)$, afetadas por erros de mensuração denotados por $\left(u_{t}, e_{t}\right)$, e que, em se lhes dando uma estrutura aditiva, resulta no seguinte modelo estatístico:

$$
\begin{aligned}
X_{t} & =x_{t}+u_{t}, \\
Y_{t} & =\alpha+\beta x_{t}+e_{t}, \quad t=1, \ldots, n
\end{aligned}
$$


Admitimos, neste trabalho, que o vetor aleatório (erros de mensuração), $\left(u_{t}, e_{t}\right)$, segue uma distribuição normal bivariado independente e identicamente distribuído com vetor de médias zero e matriz de covariâncias $\operatorname{diag}\left(\sigma_{u u}, \sigma_{e e}\right)$; ou seja:

$$
\left(u_{t}, e_{t}\right) \sim \operatorname{IIDN}\left((0,0) ; \operatorname{diag}\left(\sigma_{u u}, \sigma_{e e}\right)\right) .
$$

Usualmente, $x_{t}$ admite duas versões ou modelagem. A primeira, quando $x_{t}$ é uma v.a. com média $\mu_{x}$ e variância $\sigma_{x x}$; neste caso, o modelo é conhecido na literatura estatística como estrutural. A segunda versão considera $x_{t}, t=1, \ldots, n$, constantes desconhecidas, não-aleatórias, denominadas, na literatura, parâmetros incidentais; e o modelo é conhecido como funcional (veja Fuller (1987) ou Kendal e Stuart (1979)). Como exemplo de uma situação onde a variável explanatória $x_{t}$ não pode ser observada diretamente, consideremos a relação entre a produção de milho e quantidade de nitrogênio disponível no solo. Assumimos que a relação (1.1) é uma aproximação adequada entre a produção e a quantidade de nitrogênio. O coeficiente $\beta$ é a quantidade de produção adicionada quando o nitrogênio aumenta de uma unidade. Para estimar o nitrogênio disponível no solo é necessário amostrar o solo de uma parcela experimental e desenvolver análises laboratoriais da amostra selecionada. Como resultado da amostragem e da análise laboratorial nós não observamos $x_{t}$, mas obtemos apenas uma estimativa. Conseqüentemente, representamos o nitrogênio observado por $X_{t}$, que satisfaz (1.2), e $u_{t}$ é o erro de mensuração introduzido pela amostragem e análise laboratorial. A descrição da coleção dos dados permite duas interpretações dos verdadeiros valores de $x_{t}$, que denotaremos por $x_{t}^{0}, t=1, \ldots, n$. Na primeira, supomos que as parcelas experimentais constituem um conjunto de campos experimentais administrado por uma estação experimental, de maneira que a variação dos níveis de nitrogênio do solo, em diferentes campos, é controlada. Em tais situações trataríamos os verdadeiros, mas deconhecidos, níveis de nitrogênio em diferentes campos, como uma variável fixa. Por outro lado, se os campos forem, por exemplo, uma amostra aleatória dos campos de fazendas do estado de Iowa, os verdadeiros valores de nitrogênio no solo poderiam ser tratados como variáveis aleatórias. Este exemplo aparece, literalmente, em Fuller (1987). Outros exemplos podem ser encontrados, entre outros, em Gimenez (1997). 


\subsubsection{Formalização e Notações do Modelo Estrutural Normal}

Consideramos o modelo estatístico $(\Omega, \mathcal{A}, \mathcal{P})$ onde $\left.\mathcal{P}=\mathcal{P}\left\{\mathcal{P}_{\theta}, \theta \in \Theta \subseteq \mathbf{R}^{\mathbf{P}}\right\}\right)$, ou seja, $\Theta$, espaço paramétrico, é um retângulo de $\mathbf{R}^{p}$. O espaço amostral $\Omega \subseteq \mathbf{R}^{m \times n}$ é um conjunto de pontos que representam as amostras; e $\mathcal{A}=\mathcal{B}^{m \times n}=\mathcal{B}\left(\mathbf{R}^{m \times n}\right)$ são os borelianos de $\mathbf{R}^{m \times n}$, $\sigma$-álgebra gerada por $\mathcal{F}=\left\{A \subset \mathbf{R}^{m \times n}, A\right.$ aberto $\}$ tal que $P_{\theta}(A)$ esteja bem definida. A função densidade conjunta da amostra, que é a derivada de Radon-Nykodyn de $P_{\theta}$ em relação à medida de Lebesgue em $\mathbf{R}^{n}, \mu,(\mathcal{P}<<\mu)$, será denotada por $f(., \theta)$. A função de verossimilhança é uma função $L: \Theta \times \Omega \longrightarrow \mathbf{R}$, não negativa, dada por $L(\theta, \mathrm{x})=k f(\mathrm{z}, \theta)$, onde $k$ é um fator de proporcionalidade. $\mathrm{O}$ estimador de máxima verossimilhança do verdadeiro valor do parâmetro $\theta^{(0)} \in \Theta$ é uma função $\hat{\theta} \longrightarrow \Theta, \mathcal{A}$-mensurável, satisfazendo: $L(\hat{\theta}(\mathbf{z}), \mathbf{z})=\sup _{\theta \in \Theta} L(\theta, \mathbf{z}),[\mu]$.

Representemos por $\left\{\mathrm{z}_{t}\right\}_{t \geq 1}$ uma seqüência de vetores aleatórios observáveis independentes e $\theta=\left(\theta_{1}, \ldots, \theta_{p}\right)$ o vetor de parâmetros estruturais, que devem ser estimados. Dada uma amostra aleatória de tamanho $n$ representada por $\mathbf{Z}=\left\{\mathbf{Z}_{1}, \ldots, \mathbf{Z}_{n}\right\}$, então $\left\{\mathcal{P}_{\theta}^{(n)}\right\}_{\theta \in \Theta}$ é a família de distribuições de $Z$, portanto $Z$ é uma matriz aleatória em $\mathbf{R}^{m \times n}$ e $f_{\mathbf{Z}}(\mathbf{z}, \theta)=\prod_{t=1}^{n} f_{\mathbf{Z}_{t}}\left(\mathbf{z}_{t}, \theta\right)$ é a sua função densidade conjunta. Nos modelos considerados $P_{\theta}=N_{2}(\mathbf{m}, \Sigma)$, vale dizer, $f\left(\mathbf{z}_{t}, \theta\right)$ é uma função densidade normal bivariada, com vetor de médias $\mathrm{m}$ e matriz de covariâncias $\Sigma$. Neste trabalho, consideramos a primeira versão de $x_{t}$ sob normalidade, independentes entre si e também do vetor de erros de mensuração. Conseqüentemente, temos a seguinte suposição para o modelo estrutural:

$$
\left(x_{t}, u_{t}, e_{t}\right)^{\prime} \sim \mathrm{N}_{3}\left(\left(\mu_{x}, 0,0\right)^{\prime} ; \operatorname{diag}\left(\sigma_{x x}, \sigma_{u u}, \sigma_{e e}\right)\right), t=1, \ldots, n
$$

Supomos ainda que a matriz $\operatorname{diag}\left(\sigma_{x x}, \sigma_{u u}, \sigma_{e e}\right)$ é positiva definida e $\mu_{x} \in \mathbf{R}$. Logo, o modelo estatístico para as variáveis observáveis, $\left(X_{t}, Y_{t}\right)$, considerando a estrutura aditiva de erros, é:

$$
\left(X_{t}, Y_{t}\right) \sim \operatorname{NIID}\left[\left(\begin{array}{c}
\mu_{x} \\
\alpha+\beta \mu_{x}
\end{array}\right),\left(\begin{array}{cc}
\sigma_{u u}+\sigma_{x x} & \beta \sigma_{x x} \\
\beta \sigma_{x x} & \beta^{2} \sigma_{x x}+\sigma_{e e}
\end{array}\right)\right] .
$$


Neste modelo, sabe-se que o parâmetro de interesse $\beta$ é não identificável, pois o modelo também não o é, quando todos os seus parâmetros são desconhecidos. A não-identificabilidade é no sentido de que dois vetores de parâmetros diferentes podem gerar a mesma distribuição de probabilidade para as variáveis observáveis $X_{t}$ e $Y_{t}$. Para mais detalhes veja, por exemplo, Fuller (1987), Rojas (1995), Nascimento (1994) ou Cabral (2000). Por isso, o estudo inferencial é feito usando:

1. suposições adicionais sobre os parâmetros

- (S-1): A variância de $u_{t}, \sigma_{u u}$, é conhecida;

- (S-2): A razão entre as variâncias dos erros de observações, $\lambda=\frac{\sigma_{e e}}{\sigma_{u u}}$ é conhecida;

- (S-3): A razão $k_{x x}=\frac{\sigma_{x x}}{\sigma_{x x}+\sigma_{u u}}$ (coeficiente de atenuação) é conhecida;

- (S-4): As variâncias $\sigma_{u u}$ e $\sigma_{e e}$ são conhecidas;

- (S-5): O intercepto $\alpha$ é conhecido (igual a zero, sem perda de generalidade).

2. uma variável instrumental correlacionada com a variável explanadora.

3. replicações das verdadeiras variáveis, $\left(x_{t}, y_{t}\right)$.

Com uma das suposições (S-1), (S-2), (S-3) ou (S-4), $\beta$ torna-se identificável e, os estimadores de máxima verossimilhança de todos os parâmetros desconhecidos podem ser obtidos analiticamente, isto é, obtemos solução fechada. Esta solução é obtida resolvendo as cinco equações resultantes das igualdades dos dois primeiros momentos da distribuiçâo de $\left(X_{t}, Y_{t}\right), t=1, \ldots, n$. O caso resultante da suposição (S-5) foi resolvido em Chan e Mak (1979b); veja também Cabral (2000). O caso (3) é tratado em Chan e Mak (1979a), que estabelecem um procedimento de estimação de máxima verossimilhança, com replicações balanceadas e iguais para as variáveis explanatória e resposta, vale dizer, para cada par $\left(x_{t}, y_{t}\right)$, "r"observações repetidas $\left(X_{t j}, Y_{t j}\right), j=1, \ldots, r$, são realizadas, para $t=1, \ldots, n$, conforme o modelo (1.5). Apresentamos, a seguir, este procedimento sob o caso geral de replicações, no qual $r_{t}$ e $s_{t}$ mensurações independentes de $x_{t}$, e $y_{t}$, respectivamente, são realizadas, ou seja, estes números, agora, variam 
com " $t$ ". Esse modelo estatístico é, assim, uma extensão daquele considerado em Chan e Mak (1979a), e, pode ser especificado como:

$$
\begin{aligned}
y_{t} & =\alpha+\beta x_{t} ; \quad X_{t j}=x_{t}+u_{t j} ; \quad Y_{t l}=y_{t}+e_{t l}, \\
t & =1, \ldots, n ; j=1, \ldots, r_{t} \text { e } \quad l=1, \ldots, s_{t}
\end{aligned}
$$

com a suposição:

$$
\left(\begin{array}{c}
x_{t} \\
u_{t j} \\
e_{t l}
\end{array}\right) \sim \mathrm{N}_{3}\left[\left(\begin{array}{c}
\mu_{x} \\
0 \\
0
\end{array}\right),\left(\begin{array}{ccc}
\sigma_{x x} & 0 & 0 \\
0 & \sigma_{u u} & 0 \\
0 & 0 & \sigma_{e e}
\end{array}\right)\right] .
$$

Ao conjunto de equações (1.6) e suposição (1.7) chamamos de Modelo Estrutural Normal Bivariada com replicações não-balanceadas das variáveis explanatória e respostas $\left(x_{t}\right.$ e $\left.y_{t}\right)$, que denominaremos, simplificadamente, por Modelo Estrutural Normal nãoBalanceado. A estratégia adotada em Chan e Mak (1979a) é a de maximizar a densidade conjunta dos dados observados, $\left(X_{t j}, Y_{t j}\right), j=1, \ldots, r_{t}, l=1, \ldots, s_{t} \quad \mathrm{e}$ $t=1, \ldots, n$, em relação a todos os parâmetros desconhecidos. É com os casos particulares - relativamente ao número de replicações - do modelo descrito por (1.6) e (1.7) que trabalhamos ao longo dos Capítulos 2 e 3 .

\section{Notações do Modelo Estrutural Normal}

Sempre quando foi possível mantivemos as notações dos artigos que originaram esses estudos; e em grande parte elas são comuns aos dois modelos utilizados ao longo desta tese: Normal Estrutural e Funcional. Os vetores das observações replicadas serão denotados por:

$$
\begin{aligned}
\mathrm{X}_{\mathrm{t}}^{\prime} & =\left(X_{t 1}, X_{t 2}, \ldots, X_{t r_{t}}\right), \\
\mathrm{Y}_{\mathrm{t}}^{\prime} & =\left(Y_{t 1}, Y_{t 2}, \ldots, Y_{t s_{t}}\right), \\
\mathrm{Z}_{\mathrm{t}}^{\prime} & =\left(\mathrm{X}_{\mathrm{t}}^{\prime}, \mathrm{Y}_{\mathrm{t}}^{\prime}\right)
\end{aligned}
$$


Segue-se de (1.7) que a distribuição conjunta de $\mathbf{Z}_{t}$ é dada por $\mathbf{Z}_{t} \sim \mathrm{N}_{r_{t}+s_{t}}(\mathbf{m}, \mathbf{V})$, onde:

$$
\mathbf{m}=\left(\begin{array}{c}
\mu_{x} \mathbf{1}_{r_{t} 1} \\
\left(\beta_{o}+\beta_{1} \mu_{x}\right) \mathbf{1}_{s_{t} 1}
\end{array}\right)
$$

e

$$
\mathrm{V}=\Sigma+\sigma_{x x}\left[\begin{array}{c}
\mathbf{1}_{r_{t} 1} \\
\beta_{1} 1_{s_{t} 1}
\end{array}\right]\left[\begin{array}{cc}
1_{1 r} ; & \beta_{1} 1_{s_{t} 1}
\end{array}\right]
$$

Dada uma amostra de tamanho " $n$ " de $\mathrm{Z}_{t}$, o log-verossimilhança é:

$$
l=\ln L=-\frac{n\left(r_{t}+s_{t}\right)}{2} \ln 2 \pi-\frac{1}{2} n \ln |\operatorname{det} \mathbf{V}|-\frac{1}{2} \sum_{t=1}^{n}\left(\mathbf{Z}_{t}-\mathbf{m}\right)^{\prime} \mathbf{V}^{-1}\left(\mathbf{Z}_{t}-\mathbf{m}\right) .
$$

As estatísticas suficientes geradas por este modelo de replicação não balanceada são apresentadas a seguir.:

$$
\begin{aligned}
\bar{X}_{t .} & =\frac{\sum_{j=1}^{r_{t}} X_{t j}}{r_{t}}, \quad \bar{Y}_{t .}=\frac{\sum_{l=1}^{s_{t}} Y_{t l}}{s_{t}}, \\
\bar{X}_{. .} & =\frac{\sum_{t=1}^{n} \sum_{j=1}^{r_{t}} X_{t j}}{\sum_{t=1}^{n} r_{t}}, \quad \bar{Y} . .=\frac{\sum_{t=1}^{n} \sum_{l=1}^{s_{t}} Y_{t l}}{\sum_{t=1}^{n} s_{t}}, \\
S_{X X} & =\frac{\sum_{t=1}^{n}\left[\frac{\sum_{j=1}^{r_{t}}\left(X_{t j}-\bar{X}_{. .}\right)}{r_{t}}\right]^{2}}{n}=\frac{\sum_{t=1}^{n}\left(\bar{X}_{t .}-\bar{X}_{. .}\right)^{2}}{n}, \\
S_{Y Y} & =\frac{\sum_{t=1}^{n}\left[\frac{\sum_{l=1}^{s_{t}}\left(Y_{t l}-\bar{Y}_{. .}\right)}{s_{t}}\right]^{2}}{n}=\frac{\sum_{t=1}^{n}\left(\bar{Y}_{t .}-\bar{Y}_{. .}\right)^{2}}{n}, \\
t_{X X} & =\frac{\sum_{t=1}^{n} \sum_{j=1}^{r_{t}}\left(X_{t j}-\bar{X}_{. .}\right)^{2}}{\sum_{t=1}^{n} r_{t}}, \\
t_{Y Y} & =\frac{\sum_{t=1}^{n} \sum_{l=1}^{s_{t}}\left(Y_{t l}-\bar{Y}_{. .}\right)^{2}}{\sum_{t=1}^{n} s_{t}}, \\
S_{X Y} & =n^{-1} \sum_{t=1}^{n}\left[\frac{\sum_{l=1}^{s_{t}}\left(Y_{t l}-\bar{Y}_{. .}\right)}{s_{t}} \frac{\sum_{j=1}^{r_{t}}\left(X_{t j}-\bar{X}_{. .}\right)}{r_{t}}\right]=\frac{\sum_{t=1}^{n}\left(\bar{X}_{t .}-\bar{X}_{. .}\right)\left(\bar{Y}_{t .}-\bar{Y}_{. .}\right)}{n} .
\end{aligned}
$$


Note-se que o número de estatísticas suficientes independentes (resultantes de seis equações independentes) coincide com o número de parâmetros do modelo, $\theta=\left(\alpha, \beta, \mu_{x}, \sigma_{x x}, \sigma_{\text {: }}\right.$ o que torna o modelo identificável.

\subsubsection{Formalização do Modelo Funcional Normal}

A segunda versão do modelo considera $x_{t}, t=1, \ldots, n$, como quantidades fixas (parâmetros incidentais). Neste caso, as condições usuais de regularidade não se cumprem, pois as observações não são identicamente distribuídas; e a log-verossimilhança não é finita, sob certas condições. Uma das suposições adicionais sobre os parâmetros apresentadas adiante, garante a existência dos estimadores de máxima verossimilhança dos parâmetros estruturais do modelo funcional; para mais detalhes veja, por exemplo, Kendall e Stuart (1979, Cap.29). Essas suposições adicionais sobre os parâmetros são as mesmas adotadas para o Modelo Estrutural:

- $\left(S_{f^{-}}\right):$a variância $\sigma_{u u}$ é conhecida;

- $\left(S_{f}-2\right)$ a variância $\sigma_{e e}$ é conhecida;

- $\left(S_{f}-3\right):$ as variâncias $\sigma_{u u}$ e $\sigma_{e e}$ são conhecidas;

- $\left(S_{f}-4\right):$ a razão entre as variâncias dos erros de observações é conhecida $(\lambda=$ $\left.\frac{\sigma_{e e}}{\sigma_{e e}}\right)$.

Como no Modelo Estrutural, na nossa proposta, as replicações das verdadeiras variáveis $\left(x_{t}\right.$ e $\left.y_{t}\right)$ constituem um expediente que dispensará o uso das suposições adicionais. As condições de regularidades e restrições sobre os parâmetros incidentais, impostas para a validade dos teoremas que garantam os resultados assintóticos dos estimadores - dadas em Mak (1982), que usa a suposição $\left(S_{f}-4\right)$ - são apresentadas no Apêndice B.3.

\section{Formalização e Notações do Modelo Funcional Normal}

Sejam:

$\left(Z_{t}\right)_{t \geq 1}$ : seqüência de vetores aleatórios observados independentes, $\theta=\left(\theta_{1}, \ldots, \theta_{p}\right)^{\prime}$ : vetor paramétrico estrutural, 
$x_{t}, t=1, \ldots, n$, são os parâmetros incidentais.

Portanto, temos os seguinte espaço paramétrico: $\left(\Omega, \chi_{t}\right)$, com $\theta \in \Omega \subset \mathbf{R}^{p}$ e $x_{t} \in \chi_{t} \subseteq \mathbf{R}$.

O modelo estatístico pode ser formalizado como no caso estrutural, mediante a substituição do espaço paramétrico $\Omega$ pelo par $\left(\Omega, \chi_{t}\right)$. Dada uma amostra de tamanho $\mathrm{n}$ de $\mathbf{Z}_{t}$, a log-verossimilhanca é:

$$
\sum_{t=1}^{n} \ln f_{t}\left(\mathbf{Z}_{t} ; \theta, x_{t}\right) .
$$

Admitimos que para cada "t"e um dado " $\theta$ " existe um estimador $g_{t}\left(\mathbf{Z}_{t}, \theta\right)$, de $x_{t}$, dependente de $\theta$. Em particular, $g_{t}\left(\mathbf{Z}_{t}, \theta\right)$ pode ser um EMV condicional de $x_{t}$ dado $\theta$. Substituindo $x_{t}$ por $g_{t}\left(\mathbf{Z}_{t}, \theta\right)$, na log-verossimilhança (1.10), obtemos:

$$
\sum_{t=1}^{n} \ln f_{t}\left(\mathbf{Z}_{t} ; \theta, g_{t}\left(\mathbf{Z}_{t}, \theta\right)\right)=\sum_{t=1}^{n} h_{t}\left(\mathbf{Z}_{t}, \theta\right) \text {. }
$$

As outras notações são as mesmas do Modelo Estrutural. Os casos particulares deste modelo serão investigados no Capítulo 4.

\subsection{Objetivos da tese e sua organização.}

No Capítulo 2, a técnica de replicações balanceadas e iguais $\left(r_{t}=s_{t}=r\right)$ para as variáveis do modelo - desenvolvida em Chan e Mak (1979a), com o intuito de estabelecer um método de estimação de máxima verossimilhança (EMV) dos parâmetros de um modelo estrutural normal bivariado, na ausência de suposições identificadoras do modelo (Seção 1.3.1) - será estendida para os seguintes casos:

1. replicação balanceada só da variável explanatória, ou seja, $r_{t}=r, s_{t}=1$,

2. replicação não-balanceada só da variável explanatória, ou seja, $r_{t} \geq 1$, $s_{t}=1$,

3. replicações balanceadas da variável explanatória, ou seja, $r_{t}=r$, e da resposta, $s_{t}=s$ 
No Capítulo 3, os tipos de replicações acima serão aplicadas para obtermos os estimadores alternativos de $\beta$, sob o mesmo Modelo Estrutural Normal, ao estimar os parâmetros ou funções de parâmetros envolvidos nas condições de identificabilidade do modelo. Portanto, trabalharemos somente com os EMVs de $\beta$ gerados a partir destas condições (ver Fuller, 1987, Cap-1). Mais especificamente, usamos as replicações balanceadas para estimar:

(a) $\sigma_{u u}$, ou seja, sob a condição de identificabilidade S-1,

(b) $\lambda=\frac{\sigma_{u u}}{\sigma_{u u}}$, ou seja, sob a condição de identificabilidade S-2.

(c) fator de atenuação $\left(k_{x x}\right)$, ou seja, sob a condição S-4,

e com estes estimadores obter os estimadores alternativos de $\beta$ ao substituir as "condições de identificabilidade" nos respectivos EMVs de $\beta$, obtidos sob a égide delas. Para o caso de replicações não-balanceadas, consideramos somente a condição (S-1), ou seja, consideramos dois estimadores para o parâmetro $\sigma_{u u}$, que denotamos por $S u u_{1 n}$ e $S u u_{2 n}$. O objetivo primordial deste capítulo é o de estabelecer estimadores alternativos de $\beta$ que possam "competir" com os EMVs obtidos analiticamente, via replicações balanceadas, e os obtidos pelo algoritmo EM (replicações não-balanceadas). Tais estimadores "melhorados"são obtidos através do estimador alternativo de $\beta$, gerado pelo estimador $S u u_{2 n}$ de $\sigma_{u u}$ (o estimador $S u u_{2 n}$ é equivalente ao obtido em Fuller (1995), pelo método de estimação de análise de variância), denotado por $\widehat{\beta}_{N B 3}$. Para este aplicar-se-á um tratamento especial, que o corrigirá dos vícios de primeiro grau e melhorará as propriedades dos primeiros momentos. Posteriormente, ele também desempenhará um papel importante como um estimador preliminar, na construção de pesos, que gerarão estimadores alternativos mais elaborados de $\beta$, que incorporam as ponderações devidas às replicações. Investigamos, também, o comportamento assintótico de $\widehat{\beta}_{N B 3}$. Estudos comparativos dos estimadores melhorados com os EMVs obtidos pelas replicações balanceadas e não-balanceadas são realizados no final do capítulo.

No Capítulo 4, a técnica de replicações de variáveis junto com as condições de regularidade dadas em Mak (1982) são utilizadas para obter os EMVs dos parâmetros estruturais do Modelo Funcional Normal na ausência de suposições adicionais (Mak usa 
a condição $\left(S_{f}-4\right)$ para obter as estimativas de máxima verossimilhança). Obtemos também a matriz de covariância assintótica dos EMVs, baseada numa fórmula dada em Mak (1982). 


\section{Capítulo 2}

\section{Modelo Estrutural com Replicações na Variável Explanatória}

\subsection{Introdução}

Neste capítulo estudamos alguns casos particulares mais relevantes, sob o ponto de vista prático, do Modelo Estrutural Normal Bivariado com replicações das variáveis explanatória e resposta, descrito pelas equações (1.6) e suposições (1.7).

O caso particular $r_{t}=s_{t}=r>1$, para qualquer $t$, ou seja, replicações balanceadas e iguais das duas variáveis, foi tratado inicialmente por Tukey (1951) e Madansky (1959) usando componentes de variância. Porém, a solução de máxima verossimilhança completa só foi obtida em Chan e Mak (1979a); o EMV de $\beta$ encontrado é raíz de um polinômio de quarto grau e os estimadores de máxima verossilhança dos outros parâmetros são obtidos subseqüentemente. Eles, também, demonstram que este polinômio tem pelo menos uma raíz real, que tende a $\beta$ quando $r \rightarrow \infty$, com probabilidade um, e apresentam uma fórmula devida a Dolby que permite a obtenção da matriz de covariâncias assintótica dos EMV dos parâmetros do modelo.

Utilizamos este procedimento ao longo deste capítulo para investigar outros casos 
particulares. Assim, na Seção 2.2 tratamos do caso $r_{t}=r>1$ e $s_{t}=1$, para qualquer $\mathrm{t}$, isto é replicações balanceadas só da variável explanatória. Na Seção 2.3 considera$\operatorname{mos} s_{t}=1$ e $r_{t} \geq 1$ variando com t, isto é, replicações não-balanceadas da variável explanatória, que não tem solução analítica, resolvendo-se via algoritmo EM. E, na Seção 2.4, consideramos o caso mais geral, que ainda permite uma solução analítica, vale dizer, quando $r_{t}=r \geq 1$ e $s_{t}=s \geq 1$. Já o caso $r_{t} / s_{t}=$ cte, para qualquer t, não tem solução analítica, pelos argumentos que serão apresentados posteriormente. Desta forma, este Capítulo apresenta várias generalizações do modelo originalmente considerado em Chan e Mak (1979a).

\subsection{Replicações apenas da variável explanatória}

Nesta seção vamos obter a solução de máxima verossimilhança sob o Modelo Estrutural Normal (1.6), para o caso particular, $r_{t}=r>1$ e $s_{t}=1$, ou seja, para cada $x_{t}$, "r" observações independentes, $X_{t j}, j=1,2, \ldots, r$, são obtidas. Neste caso, não necessitamos de informações adicionais (§1.3.2) para tornar o modelo identificável. Reescrevemos o modelo considerado como

$$
\begin{aligned}
y_{t} & =\alpha+\beta x_{t}, \\
X_{t j} & =x_{t}+u_{t j}, \\
Y_{t} & =y_{t}+e_{t}, \quad t=1, \ldots, n, \quad j=1, \ldots, r .
\end{aligned}
$$

Suposições do modelo:

$$
\left(\begin{array}{c}
x_{i} \\
u_{t j} \\
e_{t}
\end{array}\right) \sim \mathrm{NIID}_{3}\left[\left(\begin{array}{c}
\mu_{x} \\
0 \\
0
\end{array}\right),\left(\begin{array}{ccc}
\sigma_{x x} & 0 & 0 \\
0 & \sigma_{u u} & 0 \\
0 & 0 & \sigma_{e e}
\end{array}\right)\right],
$$

$t=1, \ldots, n \quad j=1, \ldots, r . \quad$ Aqui, os parâmetros $\mu_{x}, \sigma_{x x}, \sigma_{u u}, \alpha, \beta \quad e \quad \sigma_{e e}$ são desconhecidos. 


\subsubsection{Log-verossimilhança}

Reescrevendo o vetor de replicações (Sub-seção 1.3.2), para o caso considerado, temos:

$$
\mathrm{X}_{\mathrm{t}}^{\prime}=\left(X_{t 1}, X_{t 2}, \ldots, X_{t r}\right)
$$

$\mathrm{e}$

$$
\mathrm{Z}_{\mathrm{t}}^{\prime}=\left(\mathrm{X}_{t}^{\prime}, Y_{t}\right), \quad t=1, \ldots, n .
$$

Segue-se de (2.1) e (2.2) que a distribuição conjunta de $Z_{t}$ é dada por $Z_{t} \sim$ $\mathrm{N}_{r+1}(\mathrm{~m}, \mathrm{~V})$, com:

$$
\begin{gathered}
\mathbf{m}=\left(\begin{array}{c}
\mu_{x} \mathbf{1}_{r 1} \\
\alpha+\beta \mu_{x}
\end{array}\right), \\
\mathbf{V}=\Sigma+\sigma_{x x}\left(\begin{array}{c}
\mathbf{1}_{r 1} \\
\beta
\end{array}\right)\left(\begin{array}{ll}
\mathbf{1}_{1 r} & \beta
\end{array}\right) \quad \text { e } \quad \boldsymbol{\Sigma}=\left(\begin{array}{cc}
\sigma_{u u} \mathbf{I}_{r} & \mathbf{0}_{r 1} \\
\mathbf{0}_{1 r} & \sigma_{e e}
\end{array}\right) .
\end{gathered}
$$

Portanto, temos:

$$
\mathbf{Z}_{t} \sim \operatorname{NIID}_{r+1}\left[\left(\begin{array}{c}
\mu_{x} \mathbf{1}_{r 1} \\
\alpha+\beta \mu_{x}
\end{array}\right),\left(\begin{array}{cc}
\sigma_{u u} \mathbf{I}_{r}+\sigma_{x x} \mathbf{1}_{r r} & \beta \sigma_{x x} \mathbf{1}_{r 1} \\
\beta \sigma_{x x} \mathbf{1}_{1 r} & \beta^{2} \sigma_{x x}+\sigma_{e e}
\end{array}\right)\right] .
$$

Dada uma amostra de tamanho $\mathrm{n}$ de $\mathrm{Z}_{t}$, obtemos a seguinte função de verossimilhança:

$$
\mathbf{L}=\prod_{t=1}^{n} f_{\mathbf{Z}_{t}}\left(\mathbf{z}_{t}\right)=\prod_{t=1}^{n}(2 \pi)^{-\left(\frac{(r+1)}{2}\right)}(\operatorname{det} \mathbf{V})^{-\frac{1}{2}} \exp \left(-\frac{1}{2}\left[\left(\mathbf{z}_{t}-\mathbf{m}\right)^{\prime} \mathbf{V}^{-1}\left(\mathbf{z}_{t}-\mathbf{m}\right)\right]\right)
$$

Logo, o log-verossimilhança pode ser escrito como

$$
\mathbf{l}=\ln L=-\frac{n(r+1)}{2} \ln 2 \pi-\frac{1}{2} n \ln |\operatorname{det} \mathbf{V}|-\frac{1}{2} \sum_{t=1}^{n}\left(\mathbf{Z}_{t}-\mathbf{m}\right)^{\prime} \mathbf{V}^{-1}\left(\mathbf{Z}_{t}-\mathbf{m}\right)
$$

Como vimos em (2.3),

$$
\mathrm{V}=\left(\begin{array}{cc}
\sigma_{u u} \mathbf{I}_{r}+\sigma_{x x} \mathbf{1}_{r r} & \beta \sigma_{x x} \mathbf{1}_{r 1} \\
\beta \sigma_{x x} \mathbf{1}_{1 r} & \beta^{2} \sigma_{x x}+\sigma_{e e}
\end{array}\right) .
$$


Usando Muirhead (1982, Teo-3.5 e A.5.3), obtemos:

$$
\operatorname{det}(\mathbf{V})=a \sigma_{x x}\left(\sigma_{u u}\right)^{r} \sigma_{e e},
$$

onde $\quad a=\sigma_{x x}^{-1}+r \sigma_{u u}^{-1}+\beta^{2} \sigma_{e e}^{-1}, \quad \mathrm{e}$

$$
\mathbf{V}^{-1}=\left(\begin{array}{cc}
\sigma_{u u}^{-1} \mathbf{I}_{r}-a^{-1} \sigma_{u u}^{-2} 1_{r r} & -a^{-1} \beta \sigma_{u u}^{-1} \sigma_{e e}^{-1} 1_{r 1} \\
-a^{-1} \beta \sigma_{e e}^{-1} \sigma_{u u}^{-1} \mathbf{1}_{1 r} & \sigma_{e e}^{-1}-a^{-1} \beta^{2} \sigma_{e e}^{2}
\end{array}\right)
$$

De (2.8) e fazendo algumas manipulações algébricas, temos, também, que:

$$
\begin{array}{cl}
\left(\mathbf{Z}_{t}-\mathbf{m}\right)^{\prime} \quad \mathbf{V}^{-1} & \left(\mathbf{Z}_{t}-\mathbf{m}\right)=\sigma_{u u}^{-1} \sum_{j=1}^{r}\left(X_{t j}-\mu_{x}\right)^{2}+\sigma_{e e}^{-1}\left(Y_{t}-\alpha-\beta \mu_{x}\right)^{2}- \\
& -a^{-1}\left[\sigma_{u u}^{-1} \sum_{j=1}^{r}\left(X_{t j}-\mu_{x}\right)+\beta \sigma_{e e}^{-1}\left(Y_{t}-\alpha-\beta \mu_{x}\right)\right]^{2} .
\end{array}
$$

Substituindo os resultados dos cálculos acima (2.6), (2.7) e (2.9), no logaritmo da função de verossimilhança (2.5), obtemos:

$$
\begin{aligned}
l= & -\left(\frac{n(r+1)}{2}\right) \ln 2 \pi-\frac{n}{2} \ln \sigma_{x x}-\frac{n r}{2} \ln \sigma_{u u}-\frac{n}{2} \ln \sigma_{e e}-\frac{n}{2} \ln a- \\
& -\frac{1}{2} \sum_{t=1}^{n}\left(h_{t}-c_{t}^{2} a^{-1}\right),
\end{aligned}
$$

com

$$
\begin{aligned}
h_{t} & =\sigma_{u u}^{-1} \sum_{j=1}^{r}\left(X_{t j}-\mu_{x}\right)^{2}+\sigma_{e e}^{-1}\left(Y_{t}-\alpha-\beta \mu_{x}\right)^{2}, \\
c_{t} & =\sigma_{u u}^{-1} \sum_{j=1}^{r}\left(X_{t j}-\mu_{x}\right)+\beta \sigma_{e e}^{-1}\left(Y_{t}-\alpha-\beta \mu_{x}\right), \\
a & =\sigma_{x x}^{-1}+r \sigma_{u u}^{-1}+\beta^{2} \sigma_{e e}^{-1} .
\end{aligned}
$$




\subsubsection{Estimadores de Máxima Verossimilhança}

Lema 2.1 Os estimadores de máxima verossimilhança $\widehat{\alpha}$ e $\widehat{\mu}_{x}$ de $\alpha$ e $\mu_{x}$, respectivamente, quando $\beta, \sigma_{x x}, \sigma_{u u}$ e $\sigma_{e e}$ são fixados, satisfazem :

$$
\begin{aligned}
\widehat{\mu}_{x} & =\bar{X} .=\frac{\sum_{t=1}^{n} \sum_{j=1}^{r} X_{t j}}{n r}, \\
\widehat{\alpha}=\alpha+\beta \widehat{\mu}_{x} & =\bar{Y} .=\frac{\sum_{t=1}^{n} Y_{t}}{n} .
\end{aligned}
$$

\section{Prova:}

Derivando parcialmente (2.10) em relação a $\mu_{x}$ e $\alpha$ e igualando a zero obtemos:

$$
\begin{gathered}
\sum_{t=1}^{n} c_{t}=0 \\
\sum_{t=1}^{n}\left(Y_{t}-\alpha-\beta \mu_{x}\right)-\beta a^{-1} \sum_{t=1}^{n} c_{t}=0 .
\end{gathered}
$$

Das equações (2.11) e (2.12), obtém-se os estimadores de máxima verossimilhança de $\mu_{x}$ e $\alpha$ sob as condições do Lema 2.1.

De acordo com Richards (1961), para maximizar $l$, basta maximizar $l^{*}$, onde $l^{*}$ é obtido através de (2.10), substituindo $\mu_{x}$ e $\alpha+\beta \mu_{x}$ por $\bar{X}_{. .}$e $\bar{Y}$., respectivamente. Portanto, a nossa tarefa será maximizar :

$$
\begin{gathered}
l^{*}=c t e-\frac{1}{2} n \ln \sigma_{x x}-\frac{1}{2} r n \ln \sigma_{u u}-\frac{1}{2} n \ln \sigma_{e e}- \\
\left.-\frac{1}{2} n \ln a-\frac{1}{2} \sum_{t=1}^{n}\left\{h_{t}^{*}-\left(c_{t}^{*}\right)^{2} a^{-1}\right)\right\},
\end{gathered}
$$

onde

$$
h_{t}^{*}=\sigma_{u u}^{-1} \sum_{j=1}^{r}\left(X_{t j}-\bar{X}_{. .}\right)^{2}+\sigma_{e e}^{-1}\left(Y_{t}-\bar{Y} .\right)^{2} \text {, }
$$




$$
c_{t}^{*}=\sigma_{u u}^{-1} \sum_{j=1}^{r}\left(X_{t j}-\bar{X}_{. .}\right)+\beta \sigma_{e e}^{-1}\left(Y_{t}-\bar{Y} .\right)
$$

e

$$
a=\sigma_{x x}^{-1}+r \sigma_{u u}^{-1}+\beta^{2} \sigma_{e e}^{-1} .
$$

As equações de máxima verossimilhança obtidas a partir de $\frac{\partial l^{*}}{\partial \psi}=0$, para $\psi=$ $\left(\beta, \sigma_{x x}, \sigma_{u u}, \sigma_{e e}\right)^{\prime}$, são:

$$
\begin{aligned}
\frac{\partial l^{*}}{\partial \beta}=0 \longrightarrow & \beta n a^{-1}-a^{-1} \sum_{t=1}^{n} c_{t}\left(Y_{t}-\bar{Y} .\right)+\beta a_{-2} \sum_{t=1}^{n} c_{t}=0 ; \\
\frac{\partial l^{*}}{\partial \sigma_{x x}}=0 \longrightarrow & n-\sigma_{x x}^{-1} n a^{-1}-\sigma_{x x}^{-1} a^{-2} \sum_{t=1}^{n}\left(c_{t}^{*}\right)^{2}=0 ; \\
\frac{\partial l^{*}}{\partial \sigma_{u u}}=0 \longrightarrow & -n r \sigma_{u u}+n r a^{-1}+\sum_{t=1}^{n} \sum_{j=1}^{r}\left(X_{t j}-\bar{X}_{. .}\right)^{2}+ \\
& +r a^{-2} \sum_{t=1}^{n}\left(c_{t} *\right)^{2}-2 a^{-1} \sum_{t=1}^{n}\left[c_{t}^{*} \sum_{j=1}^{r}\left(X_{t j}-\bar{X}_{. .}\right)\right]=0 ; \\
\frac{\partial l^{*}}{\partial \sigma_{e e}}=0 \longrightarrow & -n \sigma_{e e}+\beta^{2} n a^{-1}+\sum_{t=1}^{n}\left(Y_{t}-\bar{Y} .\right)^{2}+ \\
& +\beta^{2} a^{-2} \sum_{t=1}^{n}\left(c_{t}^{*}\right)^{2}-2 \beta \sum_{t=1}^{n} c_{t}^{*}\left(Y_{t}-\bar{Y} .\right)=0 .
\end{aligned}
$$

Esse conjunto de equações se reduz a:

$$
\begin{aligned}
\beta^{2} S_{X Y}+\beta\left(r \lambda S_{X X}-S_{Y Y}\right)-r \lambda S_{X Y} & =0 \\
\sigma_{u u}+\sigma_{x x} & =t_{X X}, \\
\sigma_{e e}+\beta^{2} \sigma_{x x} & =S_{Y Y}
\end{aligned}
$$

e

$$
\left(r \lambda t_{X X}+S_{Y Y}\right)-\left(\beta S_{X Y}+r \lambda S_{X X}\right)=r \sigma_{e e}
$$


onde

$$
\begin{gathered}
\lambda=\frac{\sigma_{e e}}{\sigma_{u u}}, \\
S_{X X}=\frac{\sum_{t=1}^{n}\left[\frac{\sum_{j=1}^{r}\left(X_{t j}-\bar{X}_{. .}\right)}{r}\right]^{2}}{n}=\frac{\sum_{t=1}^{n}\left(\bar{X}_{t .}-\bar{X}_{. .}\right)^{2}}{n} \\
S_{Y Y}=\frac{\sum_{t=1}^{n}\left(Y_{t}-\bar{Y}_{.}\right)^{2}}{n}, \\
t_{X X}=\frac{\sum_{t=1}^{n} \sum_{j=1}^{r}\left(X_{t j}-\bar{X}_{. .}\right)^{2}}{n r}
\end{gathered}
$$

e

$$
S_{X Y}=\frac{\sum_{t=1}^{n}\left[\left(Y_{t}-\bar{Y} .\right) \sum_{j=1}^{r}\left(X_{t j}-\bar{X}_{. .}\right)\right]}{n r} .
$$

Com as suposições adotadas, que definem o espaço paramétrico, $\left(\sigma_{x x}>0, \sigma_{u u}>0\right.$, $\left.\sigma_{e e}>0, \beta \neq 0\right)$, e a suposição adicional de que $\beta S_{X X}-S_{X Y} \neq 0$, podemos deduzir, a partir das equações ( 2.18) a (2.21), a seguinte equação de segundo grau em $\beta$ :

$$
S_{X Y}\left[r S_{X X}-t_{X X}\right] \widehat{\beta}_{1}^{2}+\left[S_{Y Y}\left(t_{X X}-r S_{X X}\right)-(r-1) S_{X Y}^{2}\right] \widehat{\beta}_{1}+(r-1) S_{Y Y} S_{X Y}=0
$$

cujas raízes são:

$$
\begin{array}{r}
\widehat{\beta}_{1}^{\prime}=\frac{(r-1) S_{X Y}}{r S_{X X}-t_{X X}} \\
\widehat{\beta}_{1}^{\prime \prime}=\frac{S_{Y Y}}{S_{X Y}} .
\end{array}
$$

Das raízes acima devemos optar pela primeira dada em (2.23), que denotaremos por $\widehat{\beta}_{M V}$, pois com a segunda obteremos $\widehat{\lambda}=0$, que, por sua vez, implica $\widehat{\sigma}_{e e}=0$, ou seja, um valor fora do espaço paramétrico. Isto pode ser verificado facilmente ao substituir a raiz $\widehat{\beta}_{1}^{\prime \prime}$ na equação (2.22), desde que $S_{Y Y} S_{X X} \neq S_{X Y}^{2} \neq 0$, sendo que a última restrição se garante com probabilidade um. Substituindo a raiz $\widehat{\beta}_{1}^{\prime}$ na equação (2.22) obtemos: 


$$
\begin{aligned}
\hat{\lambda} & =\frac{\left(\widehat{\beta}_{1}^{\prime}\right)^{2} S_{X Y}-\widehat{\beta}_{1}^{\prime} S_{Y Y}}{r\left(S_{X Y}-\widehat{\beta}_{1}^{\prime} S_{X X}\right)}= \\
& =\left(\frac{r-1}{r}\right)\left[\frac{(r-1) S_{X Y}^{2}-S_{Y Y}\left(r S_{X X}-t_{X X}\right)}{\left(S_{X X}-t_{X X}\right)\left(r S_{X X}-t_{X X}\right)}\right] .
\end{aligned}
$$

Façamos

$$
W_{X X}=\frac{\sum_{t=1}^{n} \sum_{j=1}^{r}\left(X_{t j}-\bar{X}_{t .}\right)^{2}}{n r} .
$$

Da igualdade $W_{X X}=t_{X X}-S_{X X}$ podemos estabelecer, de imediato, as seguintes condições que implicam em $\widehat{\lambda}>0$ :

1) Se $t_{X X}>r S_{X X} \Longrightarrow \widehat{\lambda}>0$;

2) Se $\left\{\begin{array}{l}t_{X X}<r S_{X X} \\ e \\ S_{Y Y}>\hat{\beta} S_{X Y}\end{array} \Longrightarrow \hat{\lambda}>0\right.$.

Por outro lado temos:

Se $\left\{\begin{array}{l}t_{X X} \leq r S_{X X} \\ \mathrm{e} \\ S_{Y Y} \leq \hat{\beta} S X Y\end{array} \Longrightarrow \hat{\lambda} \leq 0\right.$,

nesse caso a estimativa de $\lambda$ cairia fora do espaço paramétrico. A obtenção de outros estimadores segue-se das equações abaixo.

Do Lema 2.1 temos

$$
\widehat{\mu}_{x}=\frac{\sum_{t=1}^{n} \sum_{j=1}^{r} X_{t j}}{n r}, \quad \widehat{\alpha}=\bar{Y} . \widehat{\beta} \widehat{\mu}_{x} .
$$

Da equação (2.21) obtemos

$$
\widehat{\sigma}_{e e}=\frac{\left(r \widehat{\lambda} t_{X X}+S_{Y Y}\right)-\left(\widehat{\beta} S_{X Y}+r \widehat{\lambda} S_{X X}\right)}{r} .
$$

Uma vez obtidas $\widehat{\sigma}_{e e}$ e $\widehat{\lambda}$ podemos obter $\widehat{\sigma}_{u u}$, ou seja, $\widehat{\sigma}_{u u}=\widehat{\sigma}_{e e} / \widehat{\lambda}$. E finalmente da equação $(2.19)$, obtemos $\widehat{\sigma}_{x x}=t_{X X}-\widehat{\sigma}_{u u}$. 


\subsubsection{Precisão dos EMV's}

Nesta seção vamos obter a matriz de covariâncias assintótica dos EMV's dos parâmetros $\mu_{x}, \alpha, \beta, \sigma_{x x}, \sigma_{u u}, \sigma_{e e}$ que é dada pela inversa da matriz de informação de Fisher.

De acordo com Chan e Mak (1979a) e considerando suas notações podemos utilizar a seguinte fórmula provada por Dolby (1976):

$$
-E\left(\frac{\partial^{2} l}{\partial \psi \partial \phi}\right)=n\left[\frac{1}{2} \operatorname{tr}\left(\mathrm{V}^{-1} \mathrm{~V}_{\psi} \mathrm{V}^{-1} \mathrm{~V}_{\phi}\right)+\mathrm{d}_{\psi}^{\prime} \mathrm{V}^{-1} \mathbf{d}_{\phi}\right]
$$

em que: $\mathrm{V}_{k}=\frac{\partial \mathrm{V}}{\partial k}, \quad \mathbf{d}_{k}=\frac{\partial \mathrm{d}}{\partial k}$ para $\quad k=\psi, \phi$. Aqui $\psi$ e $\phi$ representam os seis parâmetros e

$$
\mathbf{d}=\frac{\sum_{t=1}^{n} \mathbf{d}_{t}}{n}
$$

$\operatorname{com} \mathbf{d}_{t}^{\prime}=\mathbf{Z}_{t}^{\prime}-\mathbf{m}^{\prime}=\left[\left(X_{t 1}-\mu_{x}\right), \ldots,\left(X_{t r}-\mu_{x}\right), \bar{Y} .-\left(\alpha+\beta \mu_{x}\right)\right]$. Podemos expressar $\mathrm{d}^{\prime}$ da seguinte forma:

$$
\mathbf{d}^{\prime}=\left(\bar{X}^{\prime}-\mu_{x} \mathbf{1}_{1 r}, \bar{Y} .-\left(\alpha+\beta \mu_{x}\right)\right)
$$

$\operatorname{com} \bar{X}_{. r}^{\prime}=\left(\bar{X}_{.1}, \ldots, \bar{X}_{. r}\right)$ e $\bar{X}_{. j}=\frac{\sum_{t=1}^{n} X_{t j}}{n}$ para $j=1, \ldots, r$. Derivando $\mathrm{d}$ e V em relação aos parâmetros obtêm-se os seguintes resultados:

$$
\begin{gathered}
\mathbf{d}_{\beta}=-\left(\begin{array}{l}
\mathbf{0}_{r 1} \\
\mu_{x}
\end{array}\right), \quad \mathbf{d}_{\alpha}=-\left(\begin{array}{c}
\mathbf{0}_{r 1} \\
-1
\end{array}\right), \quad \mathbf{d}_{\mu_{x}}=-\left(\begin{array}{c}
\mathbf{1}_{r 1} \\
-\beta
\end{array}\right), \\
\mathbf{d}_{\sigma_{x x}}=\mathbf{d}_{\sigma_{u u}}=\mathbf{d}_{\sigma_{e}}=\mathbf{0}_{r+1,1}, \\
\mathrm{~V}_{\beta}=\sigma_{x x}\left[\begin{array}{ll}
\mathbf{0}_{r r} & 1_{r 1} \\
1_{1 r} & 2 \beta
\end{array}\right], \quad \mathrm{V}_{\sigma_{x x}}=\left[\begin{array}{cc}
\mathbf{1}_{r r} & \beta \mathbf{1}_{r 1} \\
\beta \mathbf{1}_{1 r} & \beta^{2}
\end{array}\right], \quad \mathrm{V}_{\sigma_{u u}}=\left[\begin{array}{cc}
\mathrm{I}_{r} & \mathbf{0}_{r 1} \\
\mathbf{0}_{1 r} & 0
\end{array}\right], \\
\mathrm{V}_{\sigma_{e e}}=\left[\begin{array}{cc}
\mathbf{0}_{r r} & \mathbf{0}_{r 1} \\
\mathbf{0}_{1 r} & 1
\end{array}\right], \quad \mathrm{V}_{\beta o}=\mathrm{V}_{\mu x}=\mathbf{0}_{r+1, r+1} .
\end{gathered}
$$

Substituindo esses resultados na fórmula (2.26) obtemos a matriz de informação de Fisher em relação ao vetor de parâmetros $\left(\alpha, \mu_{x}, \beta, \sigma_{x x}, \sigma_{u u}, \sigma_{e e}\right)^{\prime}$ : 


$$
\mathbf{I}_{F}=n\left[\begin{array}{ccc}
p_{\beta \beta}+\mu_{x}^{2} v & \mathbf{q}_{12}^{\prime} & \mathbf{t}^{\prime} \\
\mathbf{q}_{21} & \mathbf{Q}_{22} & \mathbf{0}_{23} \\
\mathbf{t}_{31} & \mathbf{0}_{32} & \mathbf{T}_{33}
\end{array}\right],
$$

onde os índices nas matrizes indicam as suas dimensões e:

$$
\begin{aligned}
& p_{\beta \beta}=2 c\left(r a \sigma_{x x}+\frac{2 \beta^{2}}{\lambda}\right), \quad v=\frac{1}{\sigma_{e e}}-\frac{\beta^{2}}{a \sigma_{e e}^{2}}, \quad c=\frac{1}{2} a^{-2} \sigma_{u u}^{-1} \sigma_{e e}^{-1}, \\
& a=r \sigma_{u u}^{-1}+\sigma_{x x}^{-1}+\beta^{2} \sigma_{e e}^{-1}, \quad \mathbf{q}^{\prime}=\left(\mu_{x} v, \frac{\mu_{x} \beta}{\sigma_{e e} \sigma_{x x} a}\right), \mathbf{Q}=\left(\begin{array}{cc}
v & \frac{\beta}{a \sigma_{e e} \sigma_{x x}} \\
\beta & \frac{a-\sigma_{x x}^{-1}}{\sigma_{x x}}
\end{array}\right), \\
& \mathbf{t}^{\prime}=c\left(2 \beta\left(r+\beta^{2} \lambda^{-1}\right) \sigma_{x x}^{-1}, \quad-2 r \beta \sigma_{u u}^{-1}, \quad \frac{2 \beta}{\lambda}\left(a-\beta^{2} \sigma_{e e}^{-1}\right)\right) \\
& \mathbf{T}=c\left[\begin{array}{ccc}
\sigma_{e e} \sigma_{u u}\left(\frac{a-\sigma_{x x}^{-1}}{\sigma_{x x}}\right)^{2} & \frac{r \lambda}{\sigma_{x x}^{2}} & \frac{1}{\lambda}\left(\frac{\beta}{\sigma_{x x}}\right)^{2} \\
\frac{r \lambda}{\sigma_{x x}^{2}} & r \lambda\left(a^{2}-\frac{2 a}{\sigma_{u u}}+\frac{r}{\sigma_{u u}^{2}}\right) & \frac{r \beta^{2}}{\sigma_{e e} \sigma_{u u}} \\
\frac{1}{\lambda}\left(\frac{\beta}{\sigma_{x x}}\right)^{2} & \frac{r \beta^{2}}{\sigma_{e e} \sigma_{u u}} & \frac{1}{\lambda}\left(a^{2}-\frac{2 a \beta^{2}}{\sigma_{e e}}+\frac{\beta^{4}}{\sigma_{e e}^{2}}\right)
\end{array}\right] .
\end{aligned}
$$

Vamos obter, agora, a variância assintótica de $\widehat{\beta}_{1}$. Fazendo a seguinte partição da matriz de informação de Fisher (multiplicada por $n^{-1}$ ),

$$
n^{-1} \mathbf{I}_{F}=\left[\begin{array}{cc:c}
p_{\beta \beta}+\mu_{x}^{2} v & \mathbf{q}_{12}^{\prime} & \mathbf{t}^{\prime} \\
\mathbf{q}_{21} & \mathbf{Q}_{22} & \mathbf{0}_{23} \\
\hdashline--- & --- & ---- \\
\mathbf{t}_{31} & \mathbf{0}_{32} & \mathbf{T}_{33}
\end{array}\right]
$$

e utilizando os teoremas A.5.2 e A.5.3 em Muirhead (1982), obtemos:

$$
\operatorname{AVAR}(\widehat{\beta})=\frac{1}{n} \frac{|\mathrm{T}|}{|\mathrm{P}|}
$$

onde $\operatorname{AVAR}(\widehat{\beta})$ denota variância assintótica de $\widehat{\beta}$ e

$$
\mathbf{P}=\left[\begin{array}{cc}
p_{\beta \beta} & \mathbf{t}^{\prime} \\
\mathbf{t} & \mathrm{T}
\end{array}\right] .
$$




\subsection{Replicações não-Balanceadas da Variável Explanatória}

Nesta seção vamos obter a solução de máxima verossimilhança sob o modelo considerado na seção anterior mas com o número de replicações $\left(r_{t}\right)$ variando com t e $s_{t}=1$. Como veremos, é um caso sem solução explícita para as equações de verossimilhança e será resolvido através do algoritmo EM.

\subsubsection{Log-verossimilhança}

Reconsidere a notação onde $\left(x_{t}, y_{t}\right)$ são as variáveis não observáveis, que satisfazem a relação linear:

$$
y_{t}=\alpha+\beta x_{t}, \quad t=1, \ldots, n .
$$

$\left(X_{t}, Y_{t}\right)$ : varáveis observáveis, que são as verdadeiras mais os erros aditivos $\left(u_{t}, e_{t}\right)$, ou seja,

$$
X_{t}=x_{t}+u_{t}, \quad Y_{t}=y_{t}+e_{t}, \quad t=1, \ldots, n,
$$

em que as variáveis $x_{t}, u_{t}$ e $e_{t}$ satisfazem as condições citadas em (2.2).

Com replicações de $x_{t}$, as variáveis observáveis podem ser reapresentadas como:

$\left(X_{t j}, Y_{t}\right)$, onde $X_{t j}=x_{t}+u_{t j}, j=1, \ldots, r_{t}$, e $t=1, \ldots, n$. Façamos

$$
\mathbf{Z}_{t}=\left[\begin{array}{c}
\mathbf{X}_{t} \\
Y_{t}
\end{array}\right] \sim \mathrm{N}_{r_{t}+1}\left(\mathbf{m}_{t}, \mathbf{V}_{t}\right)
$$

onde $\mathrm{X}_{t}=\left(\mathrm{X}_{t 1}, \ldots, \mathrm{X}_{t r_{t}}\right)^{\prime}$ é o vetor de replicações e $\mathrm{Z}_{t}$ é o vetor de observações;

$$
\mathbf{m}_{t}=\left[\begin{array}{c}
\mu_{x} \mathbf{1}_{r_{t} 1} \\
\alpha+\beta \mu x
\end{array}\right], \quad \mathrm{V}_{t}=\left[\begin{array}{cc}
\sigma_{u u} \mathbf{I}_{r_{t}}+\sigma_{x x} \mathbf{1}_{r_{t} r_{t}} & \beta \sigma_{x x} \mathbf{1}_{r_{t} 1} \\
\beta \sigma_{x x} \mathbf{1}_{1 r_{t}} & \beta^{2} \sigma_{x x}+\sigma_{e e}
\end{array}\right]
$$

Usando um procedimento análogo ao da seção anterior, obtém-se:

$$
\begin{gathered}
\operatorname{det}\left(V_{t}\right)=\sigma_{x x}\left(\sigma_{u u}\right)^{r_{t}} \sigma_{e e} a_{t}, \\
\mathrm{~V}_{t}^{-1}=\left[\begin{array}{cc}
\sigma_{u u}^{-1} \mathrm{I}_{r_{t}}-a_{t}^{-1} \sigma_{u u}^{-2} 1_{r_{t} r_{t}} & -a_{t}^{-1} \beta \sigma_{u u}^{-1} \sigma_{e e}^{-1} \mathbf{1}_{r_{t} 1} \\
-a_{t}^{-1} \beta \sigma_{u u}^{-1} \sigma_{e e}^{-1} 1_{1 r_{t}} & \sigma_{e e}^{-1}-a_{t}^{-1} \beta^{2} \sigma_{e e}^{-2}
\end{array}\right], t=1, \ldots, n .
\end{gathered}
$$


Substituindo os resultados acima na log-verossimilhança de uma amostra de tamanho $\mathrm{n}$ de $\mathbf{Z}_{t}$, obtém-se:

$$
l=\text { cte }-\frac{1}{2}\left(\sum_{t=1}^{n} r_{t}\right) \ln \sigma_{u u}-\frac{n}{2} \ln \sigma_{e e}-\frac{n}{2} \ln \sigma_{x x}-\frac{1}{2} \sum_{t=1}^{n} \ln a_{t}-\frac{1}{2} \sum_{t=1}^{n}\left(h_{t t}-a_{t}^{-1} c_{t t}^{2}\right),
$$

onde

$$
\begin{aligned}
h_{t t} & =\sigma_{u u}^{-1} \sum_{j=1}^{r_{t}}\left(X_{t j}-\mu_{x}\right)^{2}+\sigma_{e e}^{-1}\left(Y_{t}-\alpha-\beta \mu_{x}\right)^{2}, \\
c_{t t} & =\sigma_{u u}^{-1} \sum_{j=1}^{r_{t}}\left(X_{t j}-\mu_{x}\right)^{2}+\beta \sigma_{e e}^{-1}\left(Y_{t}-\alpha-\beta \mu_{x}\right), \\
a_{t} & =\sigma_{x x}^{-1}+r_{t} \sigma_{u u}^{-1}+\beta^{2} \sigma_{e e}^{-1} .
\end{aligned}
$$

Para obter as equações de verossimilhança a seguir, substituiremos $\alpha+\beta \mu_{x}$ por $\bar{Y}$ nas equações acima pelas razões já citadas.

\subsubsection{Equações de verossimilhança do modelo}

Derivando (2.28) em relação aos parâmetros do modelo, obtemos:

$$
\begin{aligned}
\frac{\partial l}{\partial \mu_{x}}=0 \Longrightarrow & \sum_{t=1}^{n} a_{t}^{-1} c_{t t}=0 \\
\frac{\partial l}{\partial \alpha}=0 \Longrightarrow & \alpha+\beta \mu_{x}=\bar{Y} . \\
\frac{\partial l}{\partial \beta}=0 \Longrightarrow & \beta \sum_{t=1}^{n} a_{t}^{-1}-\sum_{t=1}^{n} a_{t}^{-1} c_{t t}\left(Y_{t}-\bar{Y} .\right)+\beta \sum_{t=1}^{n} a_{t}^{-2} c_{t t}=0, \\
\frac{\partial l}{\partial \sigma_{x x}}=0 \Longrightarrow & n-\sigma_{x x}^{-1} \sum_{t=1}^{n} a_{t}^{-1}-\sigma_{x x}^{-1} \sum_{t=1}^{n}\left(a_{t}\right)^{-2}\left(c_{t t}\right)^{2}=0 \\
\frac{\partial l}{\partial \sigma_{u u}}=0 \Longrightarrow & +\sigma_{u u} \sum_{t=1}^{n} r_{t}-\sum_{t=1}^{n} r_{t} a_{t}^{-1}-\sum_{t=1}^{n} \sum_{j=1}^{r_{t}}\left(X_{t j}-\mu_{x}\right)^{2} \\
& -\sum_{t=1}^{n}\left(c_{t t}\right)^{2} r_{t}\left(a_{t}\right)^{-2}+2 \sum_{t=1}^{n}\left[a_{t}^{-1} c_{t t} \sum_{j=1}^{r_{t}}\left(X_{t j}-\mu_{x}\right)\right]=0
\end{aligned}
$$




$$
\begin{aligned}
\frac{\partial l}{\partial \sigma_{e e}}=0 \Longrightarrow & -n \sigma_{e e}+\beta^{2} \sum_{t=1}^{n} a_{t}^{-1}+\sum_{t=1}^{n}\left(Y_{t}-\bar{Y} .\right)^{2}+ \\
& +\beta^{2} \sum_{t=1}^{n}\left(c_{t}\right)^{2} a_{t}^{-2}-2 \beta \sum_{t=1}^{n} a_{t}^{-1} c_{t t}\left(Y_{t}-\bar{Y} .\right)=0 .
\end{aligned}
$$

Com a presença de $a_{t}$ nos denominadores dos termos dos somatórios sobre $t$ nas equações de verossimilhança, conclui-se, claramente, que não pode haver soluções explícitas, conforme verificado em Searle et al. (1992, pg 88) e Miller (1977). Neste caso a utilização do algoritmo EM pode se apresentar como uma alternativa a ser considerada. Isto decorre do fato de que a maximização da verossimilhança completa - que inclui os valores não observados - é em geral mais simples que a da verossimilhança observada. Na próxima seção será implementado tal algoritmo para a obtenção dos estimadores de máxima verossimilhança no caso considerado.

\subsubsection{Estimação de Máxima Verossimilhança Usando o Algoritmo EM}

\subsubsection{1 - O algoritmo EM}

O algoritmo EM, desenvolvido por Dempster,Laird e Rubin (1977), é um método cujo objetivo é encontrar $\boldsymbol{\theta},\left(\boldsymbol{\theta} \in \Theta \subseteq \mathbb{R}^{k} ; \boldsymbol{\theta}\right.$ : vetor de parâmetros $)$, que maximize a verossimilhança dos dados observados, denotada por $g(\mathbf{z} \mid \boldsymbol{\theta})$. Escolhendo apropriadamente a função de verossimilhança dos dados completos, $f(\mathbf{w} \mid \boldsymbol{\theta})$, temos então

$$
g(\mathbf{z} \mid \boldsymbol{\theta})=\int_{\Omega_{Z}} f(\mathbf{w} \mid \boldsymbol{\theta}) d w, \quad \text { com } \quad \Omega_{Z}=\{\mathbf{w}: \mathbf{z}=\mathbf{z}(\mathbf{w})\} .
$$

Aqui estamos supondo que não observamos $W$, mas alguma função de $W$, digamos, $Z=Z(W)$. O vetor de dados completos é denotado por $W$ e o vetor de dados incompletos por Z. O algoritmo EM, na sua forma geral, é como segue:

Objetivo: Achar o estimador de máxima verossimilhança (EMV) de $\boldsymbol{\theta}$, isto é, obter $\theta$ tal que:

$$
\theta=\arg \max _{\theta} g(z \mid \theta) \text {. }
$$

Defina: $\mathbf{Q}\left(\boldsymbol{\theta}^{\prime} \mid \boldsymbol{\theta}\right)=\mathbf{E}\left(\log f\left(\mathbf{W} \mid \boldsymbol{\theta}^{\prime}\right) \mid \mathrm{Z}, \boldsymbol{\theta}\right)$. O algorimto EM definirá a passagem $\theta^{p} \longrightarrow \theta^{p+1}$, da seguinte forma: 
Etapa E: Calcule $\mathbf{Q}\left(\boldsymbol{\theta} \mid \boldsymbol{\theta}^{p}\right)$.

Etapa M: Escolha $\boldsymbol{\theta}^{p+1} \in \Theta$, que maximize $\mathbf{Q}\left(\boldsymbol{\theta}^{\prime} \mid \boldsymbol{\theta}_{p}\right)$.

Tempo de Parada: Continue até que $\left|\theta^{p}-\theta^{p+1}\right|<\epsilon$ ou $\left|g\left(\mathbf{z} \mid \theta^{p}\right)-g\left(\mathbf{z} \mid \theta^{p+1}\right)\right|<\epsilon$, para algum $\epsilon>0$.

A idéia implícita no algoritmo EM é a que segue. Gostaríamos de maximizar $f(\mathbf{w} \mid \theta)$, mas não a conhecemos; ao invés disso maximizamos $E\left[f(\mathrm{~W} \mid \boldsymbol{\theta}) \mid \mathrm{Z}, \boldsymbol{\theta}^{p}\right]$, em que $\theta^{p}$ é a estimativa corrente de $\theta$ obtida na etapa $M$ da iteração anterior. Esta estimação no caso da família exponencial regular se reduz à estimação do vetor das estatísticas suficientes $\mathbf{t}(\mathbf{w})=\left(t_{1}(\mathbf{w}) \ldots t_{k}(\mathbf{w})\right)^{\prime}$; vale dizer, maximizar $\mathbf{E}\left(\log f(\mathbf{W} / \boldsymbol{\theta}) \mid \mathbf{Z}, \boldsymbol{\theta}^{p}\right)$ equivale resolver a equação $\mathbf{t}^{p}(\mathbf{w})=\mathbf{E}_{\theta}(\mathbf{t}(\mathbf{W})$ (veja mais detalhes, entre outros, em Tanner (1996) e Peres (1987)). Neste caso as etapas E e M do algoritmo podem ser reescritas como:

1. Escolha uma estimativa inicial para $\theta$, digamos $\theta^{0}$.

2. Etapa E: Estimar as estatísticas suficientes através da equação: $\mathbf{t}^{p}(\mathbf{w})=\mathbf{E}_{\boldsymbol{\theta}^{p}}(\mathbf{t}(\mathbf{W}) \mid \mathbf{Z})$, em que $\mathbf{t}(\mathbf{w})=\left(t_{1}(\mathbf{w}) \ldots t_{k}(\mathbf{w})\right)^{\prime}$ são as estatísticas suficientes de $\boldsymbol{\theta}=\left(\theta_{1} \ldots \theta_{k}\right)^{\prime}$ e $z=z(w)$.

3. Etapa M: Determinar $\boldsymbol{\theta}^{p+1}$ como solução da equação $\mathbf{E}_{\boldsymbol{\theta}^{(p+1)}}[\mathbf{t}(\mathbf{W})]=\mathbf{t}^{p}(\mathbf{w})$.

As condições que garantem a convergência do algoritmo, ou seja, que a seqüência gerada pelas etapas E e $M$ converge para um valor $\theta^{*}$ e que este valor maximiza $\log [g(Z \mid \theta)]$, isto é, que o limite desta seqüência é o EMV de $\boldsymbol{\theta}$, são dadas nos artigos de Dempster et al. (1977), Boyles (1983) e Wu (1981). Vale destacar, no entanto, um importante resultado: $\log f\left(\mathbf{z} \mid \boldsymbol{\theta}^{(p+1)}\right) \geq \log f\left(\mathbf{z} \mid \boldsymbol{\theta}^{(p)}\right)$, isto é, cada iteração do algoritmo EM aumenta a verossimilhança dos dados observados . 
2.3.3.2 - Aplicação do algoritmo EM no caso de número de replicações não constantes da variável explanatória.

O algoritmo EM pode ser usado no modelo estrutural com erros nas variáveis, com réplicas ao considerar as verdadeiras variáveis $x_{t}$ como dados perdidos. De acordo com a notação usada na subseção anterior, temos:

Dados completos: $\mathrm{W}_{t}=\left(\mathrm{X}_{t}, y_{t}, x_{t}\right)$

Dados incompletos (observados): $\mathrm{Z}_{t}=\left(\mathrm{X}_{t}, Y_{t}\right), t=1 \ldots n$.

Já obtivemos a log-verossimilhança para os dados incompletos (observados). Vamos, então, obtê-la para os dados completos. Com as suposições feitas para este modelo segue-se que a distribuição conjunta dos dados completos é:

$$
\left(\mathbf{X}_{t}, y_{t}, x_{t}\right) \sim \mathrm{N}_{r_{t}+2}\left(\left[\begin{array}{c}
\mu_{x} \mathbf{1}_{r_{t} 1} \\
\alpha+\beta \mu_{x} \\
\mu_{x}
\end{array}\right],\left[\begin{array}{ccc}
\sigma_{x x} \mathbf{1}_{r_{t} r_{t}}+\sigma_{u u} \mathrm{I}_{r_{t}} & \beta \sigma_{x x} \mathbf{1}_{r_{t} 1} & \sigma_{x x} \mathbf{1}_{r_{t} 1} \\
\beta \sigma_{x x} \mathbf{1}_{1 r_{t}} & \beta^{2} \sigma_{x x}+\sigma_{e e} & \beta \sigma_{x x} \\
\sigma_{x x} \mathbf{1}_{r_{t} 1} & \beta \sigma_{x x} & \sigma_{x x}
\end{array}\right]\right)
$$

$t=1, \ldots, n . \quad$ Portanto, a log-verossimilhança dos dados completos é:

$$
\begin{aligned}
l_{\mathbf{c}}= & -\left(\sum_{t=1}^{n} \frac{\left(r_{t}+2\right)}{2}\right) \ln 2 \pi-\frac{n}{2} \ln \sigma_{x x}-\frac{\sum_{t=1}^{n} r_{t}}{2} \ln \sigma_{u u}-\frac{n}{2} \ln \sigma_{e e} \\
& -\frac{\sigma_{u u}^{-1}}{2} \sum_{t=1}^{n} \sum_{j=1}^{r_{t}}\left(X_{t j}-x_{t}\right)^{2}-\frac{\sigma_{e e}^{-1}}{2} \sum_{t=1}^{n}\left(Y_{t}-\alpha-\beta \mu_{x}\right)^{2}-\frac{\sigma_{x x}^{-1}}{2} \sum_{t=1}^{n}\left(x_{t}-\mu_{x}\right)^{2} .
\end{aligned}
$$

\section{Obtenção das estatísticas suficientes de $\theta$}

Derivando (2.37) em relação ao vetor paramétrico $\theta=\left(\alpha, \beta, \mu x, \sigma_{x x}, \sigma_{u u}, \sigma_{e e}\right)^{\prime} \mathrm{e}$ igualando a zero, obtemos: 


$$
\begin{gathered}
\widehat{\mu}=\frac{\sum_{t=1}^{n} x_{t}}{n}, \quad \widehat{\alpha}=\bar{Y} \cdot-\widehat{\beta} \bar{x}, \quad \widehat{\beta}=\frac{\sum_{t=1}^{n}\left(x_{t}-\bar{x}\right)\left(Y_{t}-\bar{Y}\right)}{\sum_{t=1}^{n}\left(x_{t}-\bar{x}\right)^{2}}, \quad \widehat{\sigma}_{x x}=\frac{\sum_{t=1}^{n}\left(x_{t}-\bar{x}\right)^{2}}{n}, \\
\widehat{\sigma}_{u u}=\frac{\sum_{t=1}^{n} \sum_{j=1}^{r_{t}}\left(X_{t j}-x_{t}\right)^{2}}{\sum_{t=1}^{n} r_{t}}, \quad \widehat{\sigma}_{e e}=\frac{\sum_{t=1}^{n}\left(Y_{t}-\widehat{\beta}_{0}-\widehat{\beta} x_{t}\right)^{2}}{n} .
\end{gathered}
$$

Este estimadores são exatamente os de mínimos quadrados da regressão linear simples convencional, ou seja, quando $X_{t j}=x_{t}, j=1, \ldots, r_{t}$. Isto se justifica pelo fato da distribuição dos dados completos ser da família exponencial regular e o número de parâmetros coincidir com o de estatísticas suficientes $\left(\bar{x}, \bar{Y}, m_{x Y}, m_{x x}, m_{Y Y}, m_{X x}\right)^{\prime}$, onde $m_{x Y}=\frac{\sum_{t=1}^{n}\left(x_{t}-\bar{x}\right)\left(Y_{t}-\bar{Y}\right)}{n}$; os outros momentos sâo definidos analogamente.

Etapa E- Passo p.

(i) Calcular os estimadores de $V_{t}=\operatorname{Var}\left(x_{t} \mid \mathrm{X}_{t}, \mathrm{Y}_{t}\right)$ e $E_{t}=E\left(x_{t} \mid \mathrm{X}_{t}, \mathrm{Y}_{t}\right)$. No passo p, temos (Apêndice A.1) :

$$
\begin{gathered}
V_{t}^{(p)}=\frac{\sigma_{e e}^{(p)} \sigma_{x x}^{(p)} \sigma_{u u}^{(p)}}{\sigma_{u u}^{(p)}\left[\sigma_{e e}^{(p)}+\left(\beta^{(p)}\right)^{2} \sigma_{x x}^{(p)}\right]+r_{t} \sigma_{x x}^{(p)} \sigma_{e e}^{(p)}} \\
E_{t}^{(p)}=\mu_{x}^{(p)}+\frac{V_{t}^{(p)}}{\sigma_{e e}^{(p)} \sigma_{u u}^{(p)}}\left(\beta^{(p)} \sigma_{u u}^{(p)}\left[Y_{t}-\left(\alpha^{(p)}+\beta^{(p)} \mu_{x}\right)\right]+\sigma_{e e}^{(p)} \sum_{j=1}^{r_{t}}\left(X_{t j}-\mu_{x}^{(p)}\right)\right) .
\end{gathered}
$$

(ii) Estatísticas Suficientes:

$$
\begin{gathered}
S_{x}=\sum_{t=1}^{n} x_{t}, \quad S_{x x}=\sum_{t=1}^{n} x_{t}^{2}, \quad S_{x Y}=\sum_{t=1}^{n} x_{t} Y_{t}, \quad S_{x X}=\sum_{t=1}^{n}\left(x_{t} \sum_{j=1}^{r_{t}} X_{t j}\right) \\
S_{Y}=\sum_{t=1}^{n} Y_{t}, \quad S_{Y Y}=\sum_{t=1}^{n} Y_{t}^{2}, \quad S_{x x}^{(*)}=\sum_{t=1}^{n} r_{t} x_{t}^{2}, \quad S_{X X}=\sum_{t=1}^{n} \sum_{j=1}^{r_{t}} X_{t j} .
\end{gathered}
$$

(iii) Cálculos das esperanças condicionais das estatísticas suficientes. Note que

$$
\left.S_{x}^{(p)}=E\left(S_{x} \mid \mathbf{X}_{t}, Y_{t}, \theta^{(p)}\right)=E\left(\sum_{t=1}^{n} x_{t} \mid Y_{t}, \mathbf{X}_{t}, \theta^{(p)}\right)\right]=\sum_{t=1}^{n} E_{t}^{(p)}
$$


Analogamente, obtemos:

$$
S_{x x}^{(p)}=\sum_{t=1}^{n}\left[\left(E_{t}^{(p)}\right)^{2}+V_{t}^{(p)}\right], \quad S_{x Y}^{(p)}=\sum_{t=1}^{n} Y_{t} E_{t}^{(p)}, \quad S_{x X}^{(p)}=\sum_{t=1}^{n}\left[E_{t}^{(p)} \sum_{j=1}^{r_{t}} X_{t j}\right]
$$

e, de maneira similar

$$
S_{x x}^{*(p)}=\sum_{t=1}^{n} r_{t}\left[\left(E_{t}^{(p)}\right)^{2}+V_{t}^{(p)}\right] .
$$

Etapa $\mathrm{M}$ - Passo p+1.

Determinar $\theta^{(p+1)}$ como solução da equação :

$$
E_{\theta(p+1)}\left(\mathbf{t}\left(\mathbf{W}_{\mathbf{t}}\right)\right)=\mathbf{t}^{(p)}\left(\mathbf{w}_{\mathbf{t}}\right), \text { com } \mathbf{W}_{t}=\left(\mathbf{X}_{t}, Y_{t}, x_{t}\right) \text {. }
$$

Temos que $E_{\theta(p+1)}\left(S_{x}\right)=S_{x}^{(p)}$, de modo que $E_{\theta(p+1)}\left(\sum_{t=1}^{n} x_{t}\right)$

$$
=\sum_{t=1}^{n} E_{\theta(p+1)}\left(x_{t}\right)=\sum_{t=1}^{n} \mu_{x}^{(p)}=S_{x}^{(p)} \text {, portanto, temos }
$$

$$
\mu_{x}^{(p+1)}=\frac{S_{x}^{(p)}}{n} .
$$

Analogamente, obtemos:

$$
\begin{aligned}
& \beta^{(p+1)}=\frac{S_{x Y}^{(p)}-\bar{Y} S_{x}^{(p)}}{S_{x x}^{(p)}-\left(S_{x}^{(p)}\right)^{2} / n}, \\
& \alpha^{(p+1)}=\bar{Y}-\beta^{(p+1)}\left(S_{x}^{(p)} / n\right) \\
& \sigma_{u u}^{(p+1)}=\frac{\sum_{t=1}^{n} \sum_{j=1}^{r_{t}} X_{t j}^{2}-2 S_{x X}^{(p)}+\left(S_{x x}^{*}\right)^{(p)}}{\sum_{t=1}^{n} r_{t}} \\
& \sigma_{x x}^{(p+1)}=\frac{S_{x x}^{(p)}-\left(S_{x}^{(p)}\right)^{2} / n}{n} \\
& \sigma_{e e}^{(p+1)}=\quad \sum_{t=1}^{n}\left(Y_{t}-\alpha^{(p+1)}\right)^{2}-2 \beta^{(p+1)} \sum_{t=1}^{n}\left(Y_{t}-\alpha^{(p+1)}\right) E_{t}^{(p)}+\left(\beta^{(p+1)}\right)^{2} S_{x x}^{(p)} \\
& n
\end{aligned}
$$

A matriz de covariâncias assintóticas dos EMV's dos parâmetros desse modelo será calculada na próxima seção. 


\subsection{Extensão do caso Chan e Mak (1979a): $r_{t}=r$ e $s_{t}=s$.}

\subsubsection{Notações e modelo}

Nesta seção obtemos a solução de máxima verossimilhança sob o Modelo Estrutural Normal descrito por (1.6) e (1.7), considerando o caso particular em que $r_{t}=r$ e $s_{t}=s$. Ou seja, para cada $\left(x_{t}, y_{t}\right)$, r observações independentes, $X_{t j}, j=1,2, \ldots, r$, e s observações independentes, $Y_{t l}, l=1,2, \ldots, s$, são obtidas. Neste caso, também, não necessitamos de informações adicionais para tornar o modelo identificável. O modelo considerado pode ser escrito na forma:

$$
\begin{aligned}
& y_{t}=\alpha+\beta x_{t} ; \quad X_{t j}=x_{t}+u_{t j} ; \quad Y_{t l}=y_{t}+e_{t l} \\
& (t=1, \ldots, n ; \quad j=1, \ldots, r, \text { e } \quad l=1, \ldots, s)
\end{aligned}
$$

Suposição:

$$
\left(\begin{array}{c}
x_{t} \\
u_{t j} \\
e_{t l}
\end{array}\right) \sim \mathrm{N}_{3}\left[\left(\begin{array}{c}
\mu_{x} \\
0 \\
0
\end{array}\right),\left(\begin{array}{ccc}
\sigma_{x x} & 0 & 0 \\
0 & \sigma_{u u} & 0 \\
0 & 0 & \sigma_{e e}
\end{array}\right)\right]
$$

Como anteriormente, os parâmetros $\mu_{x}, \sigma_{x x}, \sigma_{u u}, \alpha, \beta$ e $\sigma_{e e}$ são desconhecidos.

\subsubsection{Log-verossimilhança}

Sejam:

$$
\begin{gathered}
\mathrm{X}_{\mathrm{t}}=\left(X_{t 1}, X_{t 2}, \ldots, X_{t r}\right)^{\prime}, \\
\mathrm{Y}_{\mathrm{t}}=\left(Y_{t 1}, Y_{t 2}, \ldots, Y_{t s}\right)^{\prime}, \\
\mathrm{Z}_{\mathrm{t}}=\left(\mathrm{X}_{\mathbf{t}}, \mathrm{Y}_{\mathrm{t}}\right)^{\prime} .
\end{gathered}
$$

Seguem-se de (2.44) e (2.45) que a distribuição conjunta de $\mathbf{Z}_{t}$ é dada por $\mathbf{Z}_{t} \sim$ $\mathrm{N}_{r+s}(\mathrm{~m}, \mathrm{~V})$, com:

$$
\mathbf{m}=\left[\begin{array}{c}
\mu_{x} 1_{r 1} \\
\left(\alpha+\beta \mu_{x}\right) 1_{s 1}
\end{array}\right]
$$




$$
\mathrm{V}=\boldsymbol{\Sigma}+\sigma_{x x}\left[\begin{array}{c}
\mathbf{1}_{r 1} \\
\beta \mathbf{1}_{s 1}
\end{array}\right]\left[\begin{array}{cc}
\mathbf{1}_{1 r x} ; & \beta \mathbf{1}_{s 1}
\end{array}\right] \quad \text { e } \quad \boldsymbol{\Sigma}=\left[\begin{array}{cc}
\sigma_{u u} \mathbf{I}_{r} & \mathbf{0}_{r s} \\
\mathbf{0}_{s r} & \sigma_{e e} \mathbf{I}_{s}
\end{array}\right]
$$

Portanto, temos

$$
\mathbf{Z}_{t} \sim \mathbf{N}_{r+s}\left(\left[\begin{array}{c}
\mu_{x} \mathbf{1}_{r 1} \\
\left(\alpha+\beta \mu_{x}\right) \mathbf{1}_{s 1}
\end{array}\right],\left[\begin{array}{cc}
\sigma_{u u} \mathbf{I}_{r}+\sigma_{x x} \mathbf{1}_{r r} & \beta \sigma_{x x} \mathbf{1}_{r s} \\
\beta \sigma_{x x} \mathbf{1}_{s r} & \beta^{2} \sigma_{x x} \mathbf{1}_{s s}+\sigma_{e e} \mathbf{I}_{s}
\end{array}\right]\right),
$$

$t=1, \ldots, n$. Dada uma amostra de tamanho $\mathrm{n}$ de $\mathrm{Z}_{t}$, obtemos a seguinte função de verossimilhança :

$$
\mathbf{L}=\mathbf{f}_{\mathbf{Z}_{t}}\left(\mathbf{z}_{t}\right)=\prod_{t=1}^{n}(2 \pi)^{-\left(\frac{(r+s)}{2}\right)}(\operatorname{det} \mathbf{V})^{-\frac{1}{2}} \exp \left(-\frac{1}{2}\left[\left(\mathbf{z}_{t}-\mathbf{m}\right)^{\prime} \mathbf{V}^{-1}\left(\mathbf{z}_{t}-\mathbf{m}\right)\right]\right) .
$$

Logo, a log-verossimilhança é

$$
l=\ln L=-\frac{n(r+s)}{2} \ln 2 \pi-\frac{1}{2} n \ln |\operatorname{det} \mathrm{V}|-\frac{1}{2} \sum_{t=1}^{n}\left(\mathbf{Z}_{t}-\mathbf{m}\right)^{\prime} \mathbf{V}^{-1}\left(\mathbf{Z}_{t}-\mathbf{m}\right)
$$

onde

$$
\mathrm{V}=\left[\begin{array}{cc}
\sigma_{u u} \mathbf{I}_{r}+\sigma_{x x} \mathbf{1}_{r r} & \beta \sigma_{x x} \mathbf{1}_{r s} \\
\beta \sigma_{x x} \mathbf{1}_{s r} & \beta^{2} \sigma_{x x} \mathbf{1}_{s s}+\sigma_{e e} \mathbf{I}_{s}
\end{array}\right]
$$

Usando o procedimento análogo ao das seções anteriores, obtemos

$$
\operatorname{det}(\mathrm{V})=a \sigma_{x x}\left(\sigma_{u u}\right)^{r} \sigma_{e e}^{s},
$$

em que $\quad a=\sigma_{x x}^{-1}+r \sigma_{u u}^{-1}+s \beta^{2} \sigma_{e e}^{-1}$.

Também

$$
\mathbf{V}^{-1}=\left[\begin{array}{cc}
\sigma_{u u}^{-1} \mathbf{I}_{r}-a^{-1} \sigma_{u u}^{-2} 1_{r r} & -a^{-1} \beta \sigma_{u u}^{-1} \sigma_{e e}^{-1} 1_{r r y} \\
-a^{-1} \beta \sigma_{e e}^{-1} \sigma_{u u}^{-1} 1_{s r} & \sigma_{e e}^{-1} \mathbf{I}_{s}-a^{-1} \beta^{2} \sigma_{e e}^{-1} 1_{s s}
\end{array}\right]
$$

$$
\left(\mathbf{Z}_{t}-\mathbf{m}\right)^{\prime} \mathbf{V}^{-1}\left(\mathbf{Z}_{t}-\mathbf{m}\right)=\left[\sigma_{u u}^{-1} \sum_{j=1}^{r}\left(X_{t j}-\mu_{x}\right)^{2}+\sigma_{e e}^{-1} \sum_{l=1}^{s}\left(Y_{t l}-\alpha-\beta \mu_{x}\right)^{2}\right]
$$




$$
-a^{-1}\left[\sigma_{u u}^{-1} \sum_{j=1}^{r}\left(X_{t j}-\mu_{x}\right)+\beta \sigma_{e e}^{-1} \sum_{l=1}^{s}\left(Y_{t l}-\alpha-\beta \mu_{x}\right)\right]^{2} .
$$

Substituindo os resultados acima na log-verossimilhança obtemos:

$$
\begin{aligned}
l= & -\left(\frac{n(r+s)}{2}\right) \ln 2 \pi-\frac{n}{2} \ln \sigma_{x x}-\frac{n r}{2} \ln \sigma_{u u}-\frac{n s}{2} \ln \sigma_{e e}-\frac{n}{2} \ln a- \\
& -\frac{1}{2} \sum_{t=1}^{n}\left(h_{t}-c_{t}^{2} a^{-1}\right)
\end{aligned}
$$

onde

$$
h_{t}=\sigma_{u u}^{-1} \sum_{j=1}^{r}\left(X_{t j}-\mu_{x}\right)^{2}+\sigma_{e e}^{-1} \sum_{l=1}^{s}\left(Y_{t l}-\alpha-\beta \mu_{x}\right)^{2}
$$

$\mathrm{e}$

$$
c_{t}=\sigma_{u u}^{-1} \sum_{j=1}^{r}\left(X_{t j}-\mu_{x}\right)+\beta \sigma_{e e}^{-1} \sum_{l=1}^{r}\left(Y_{t l}-\alpha-\beta \mu_{x}\right) .
$$

\subsubsection{Equações de verossimilhança}

Derivando (2.47) em relação aos parâmetros do modelo, obtemos:

$$
\begin{aligned}
& \frac{\partial \ln L}{\partial \mu_{x}}=0 \Longrightarrow \sum_{t=1}^{n} c_{t}=0 \\
& \frac{\partial \ln L}{\partial \alpha}=0 \Longrightarrow s \beta \sum_{t=1}^{n} c_{t}+\sum_{t=1}^{n} \sum_{l=1}^{s}\left(Y_{t l}-\alpha-\beta \mu_{x}\right)=0 .
\end{aligned}
$$

Das equações acima, obtemos

$$
\begin{aligned}
\widehat{\mu}_{x} & =\bar{X}_{. .}=\frac{\sum_{t=1}^{n} \sum_{j=1}^{r} X_{t j}}{n r}, \\
\widehat{\alpha}+\beta \widehat{\mu}_{x} & =\bar{Y}_{. .}=\frac{\sum_{t=1}^{n} \sum_{l=1}^{s} Y_{t l}}{n s},
\end{aligned}
$$


que substituidos em $\ln L$, de acordo com o Lema 2.1, resulta em:

$$
\begin{aligned}
L_{1}= & -\left(\frac{n(r+s)}{2}\right) \ln 2 \pi-\frac{n}{2} \ln \sigma_{x x}-\frac{n r}{2} \ln \sigma_{u u}-\frac{n s}{2} \ln \sigma_{e e}-\frac{n}{2} \ln a \\
& -\frac{1}{2} \sum_{t=1}^{n}\left(\widehat{h}_{t}-\left(\widehat{c}_{t}\right)^{2} a^{-1}\right),
\end{aligned}
$$

onde

$$
\widehat{h}_{t}=\sigma_{u u}^{-1} \sum_{j=1}^{r}\left(X_{t j}-\bar{X}_{. .}\right)^{2}+\sigma_{e e}^{-1} \sum_{l=1}^{s}\left(Y_{t l}-\bar{Y}_{. .}\right)^{2}
$$

e

$$
\widehat{c}_{t}=\sigma_{u u}^{-1} \sum_{j=1}^{r}\left(X_{t j}-\bar{X}_{. .}\right)+\beta \sigma_{e e}^{-1} \sum_{l=1}^{r}\left(Y_{t l}-\bar{Y}_{. .}\right) \text {. }
$$

Derivando (2.48) em relação aos outros parâmetros, obtemos:

$$
\begin{aligned}
\frac{\partial L_{1}}{\partial \beta}=0 \Longrightarrow & n s \beta+s \beta a^{-1} \sum_{t=1}^{n}\left(\widehat{c}_{t}\right)^{2}-\sum_{t=1}^{n}\left[\widehat{c}_{t} \sum_{l=1}^{s}\left(Y_{t l}-\bar{Y}_{. .}\right)\right]=0, \\
\frac{\partial L_{1}}{\partial \sigma_{x x}}=0 \Longrightarrow & -n+n \sigma_{x x}^{-1} a^{-1}+\sigma_{x x}^{-1} a^{-2} \sum_{t=1}^{n}\left(\widehat{c}_{t}\right)^{2}=0, \\
\frac{\partial L_{1}}{\partial \sigma_{u u}}=0 \Longrightarrow & -n r \sigma_{u u}+n r a^{-1}+\sum_{t=1}^{n} \sum_{j=1}^{r}\left(X_{t j}-\bar{X}_{. .}\right)^{2} \\
& +r a^{-2} \sum_{t=1}^{n}\left(\widehat{c}_{t}\right)^{2}-2 a^{-1} \sum_{t=1}^{n}\left[\widehat{c}_{t} \sum_{j=1}^{r}\left(X_{t j}-\bar{X}_{. .}\right)\right]=0, \\
\frac{\partial L_{1}}{\partial \sigma_{e e}}=0 \Longrightarrow & -n s \sigma_{e e}+s \beta^{2} n a^{-1}+\sum_{t=1}^{n} \sum_{t=1}^{n}\left(Y_{t l}-\bar{Y}_{. .}\right)^{2} \\
& +s \beta^{2} a^{-2} \sum_{t=1}^{n} \hat{c}_{t}^{2}-2 \beta a^{-1} \sum_{t=1}^{n}\left[\widehat{c}_{t} \sum_{l=1}^{s}\left(Y_{t l}-\bar{Y}_{. .}\right)\right]=0 .
\end{aligned}
$$

O conjunto de equações (2.49) - (2.52) se reduz a: 


$$
\begin{aligned}
\beta^{2} S_{X Y}+\beta\left(\frac{r}{s} \lambda S_{X X}-S_{Y Y}\right)-\frac{r}{s} \lambda S_{X Y} & =0 \\
\sigma_{u u}+\sigma_{x x} & =t_{X X}, \\
\sigma_{e e}+\beta^{2} \sigma_{x x} & =t_{Y Y}, \\
\left(r \lambda t_{X X}+s t_{Y Y}\right)-\left(s \beta S_{X Y}+r \lambda S_{X X}\right) & =(r+s-1) \sigma_{e e},
\end{aligned}
$$

em que

$$
\begin{aligned}
\lambda=\frac{\sigma_{e e}}{\sigma_{u u}}, \quad \bar{X}_{t .}=\frac{\sum_{j=1}^{r} X_{t j}}{r}, \quad \bar{Y}_{t .}=\frac{\sum_{l=1}^{s} Y_{t j}}{r} \\
S_{X X}=\frac{\sum_{t=1}^{n}\left[\frac{\sum_{j=1}^{r}\left(X_{t j}-\bar{X}_{. .}\right)}{r}\right]^{2}}{n}=\frac{\sum_{t=1}^{n}\left(\bar{X}_{t .}-\bar{X}_{. .}\right)^{2}}{n} \\
S_{Y Y}=\frac{\sum_{t=1}^{n}\left[\frac{\sum_{l=1}^{s}\left(Y_{t l}-\bar{Y}_{. .}\right)}{s}\right]^{2}}{n}=\frac{\sum_{t=1}^{n}\left(\bar{Y}_{t .}-\bar{Y}_{. .}\right)^{2}}{n} \\
t_{X X}=\frac{\sum_{t=1}^{n} \sum_{j=1}^{r}\left(X_{t j}-\bar{X}_{. .}\right)^{2}}{n r}, \\
t_{Y Y}=\frac{\sum_{t=1}^{n} \sum_{l=1}^{s}\left(X_{t l}-\bar{Y}_{. .}\right)^{2}}{n s}, \\
S_{X Y}=\frac{\sum_{t=1}^{n}\left[\sum_{l=1}^{s}\left(Y_{t l}-\bar{Y}_{. .}\right) \sum_{j=1}^{r}\left(X_{t j}-\bar{X}_{. .}\right)\right]}{n r s} .
\end{aligned}
$$

A partir das equações (2.53) - (2.56) podemos deduzir o seguinte polinômio de quarto grau:

$$
P(n, r, s)=k_{0} \beta^{4}+k_{1} \beta^{3}+k_{2} \beta^{2}+k_{3} \beta+k_{4}=0,
$$

onde

$$
\begin{aligned}
k_{0}= & s(s-1) t_{X X} S_{X Y} S_{X X}, \\
k_{1}= & \left(s\left[r W_{Y Y} S_{X X}^{2}-(s-1) t_{X X}\left(S_{Y Y} S_{X X}+S_{X Y}^{2}\right)\right]-s W_{X X} S_{X Y}^{2}\right. \\
& -(s-r) S_{X X} S_{X Y}^{2},
\end{aligned}
$$




$$
\begin{aligned}
k_{2}= & r_{4}\left[(3 s-1) S_{X Y} S_{Y Y} W_{X X}+(3 r-1) S_{X X} S_{X Y} W_{Y Y}\right] \\
& +(s-r) S_{X Y}\left(2 S_{X X} S_{Y Y}+S_{X Y}^{2}\right) \\
k_{3}= & s\left[r S_{X X}^{2} W_{Y Y}-s W_{X X} S_{Y Y}^{2}+(r-1) t_{Y Y} S_{Y Y} S_{X X}\right] \\
& +(r-1) t_{Y Y} S_{X Y}^{2}-(s-r) s S_{X Y}^{2} S_{Y Y} \\
k_{4}= & -s(r-1) t_{Y Y} S_{Y Y} S_{X Y}
\end{aligned}
$$

com

$$
W_{X X}=t_{X X}-S_{X X} \quad \text { e } \quad W_{Y Y}=t_{Y Y}-S_{Y Y} \text {. }
$$

\subsubsection{Casos particulares}

Se $r=s$ obteremos o polinônio de quarto grau em $\beta$ obtido em Chan e Mak (1979).

Se $s=1$, temos $t_{Y Y}=S_{Y Y}, \operatorname{logo} W_{Y Y}=0$ e, portanto, os coeficientes do polinômio (2.57) se reduzirão a :

$$
\begin{aligned}
k_{0}^{\prime} & =0, \\
k_{1}^{\prime} & =S_{X Y}^{2}\left(r S_{X X}-t_{X X}\right), \\
k_{2}^{\prime} & =2 S_{X Y} S_{Y Y}\left(t_{X X}-r S_{X X}\right)+(1-r) S_{X Y}^{3}, \\
k_{3}^{\prime} & =S_{Y Y}\left[S_{Y Y}\left(r S_{X X}-t_{X X}\right)+2(r-1) S_{X Y}^{2}\right], \\
k_{4}^{\prime} & =-(r-1) t_{Y Y} S_{Y Y} S_{X Y} .
\end{aligned}
$$

Conseqüentemente, temos o polinômio:

$$
\begin{aligned}
P(n, r)= & k_{1}^{\prime} \beta^{3}+k_{2}^{\prime} \beta^{2}+k_{3}^{\prime} \beta+k_{4}^{\prime}=\left(\beta S_{X Y}-S_{Y Y}\right)\left\{S_{X Y}\left(r S_{X X}-t_{X X}\right) \beta^{2}+\right. \\
& {\left.\left[t_{Y Y}\left(t_{X X}-r S_{X X}\right)-(r-1) S_{X Y}^{2}\right] \beta+(r-1) t_{Y Y} S_{X Y}\right\}, }
\end{aligned}
$$

que é equivalente ao obtido em (2.2). 


\subsubsection{Matriz de Covariâncias Assintóticas dos EMV's}

A matriz de covariância assintótica dos EMV do vetor paramétrico, como visto anteriormente, é a inversa da matriz de informação de Fisher. Usando o mesmo procedimento aplicado na Seção 2.2.3 obtemos, agora, a matriz de informação de Fisher em relação ao vetor paramétrico $\left(\alpha, \mu_{x}, \beta, \sigma_{x x}, \sigma_{u u}, \sigma_{e e}\right)^{\prime}$ para o caso $r_{t}=r$ e $s_{t}=s$. Temos, então, após uma série de manipulações algébricas que

$$
\mathbf{I}_{F}=n\left[\begin{array}{ccc}
p_{\beta \beta}^{*}+\mu_{x}^{2} v^{*} & \mathbf{q}^{*^{\prime}} & \mathbf{t}^{*^{\prime}} \\
\mathbf{q}^{*}{ }_{21} & \mathbf{Q}_{22}^{*} & \mathbf{0}_{23} \\
\mathbf{t}^{*}{ }_{31} & \mathbf{0}_{32} & \mathbf{T}^{*}{ }_{33}
\end{array}\right]
$$

em que:

$$
\begin{aligned}
& p_{\beta \beta}^{*}=2 c^{*} s\left(r a^{*} \sigma_{x x}+\frac{2 s \beta^{2}}{\lambda}\right), \quad v^{*}=\frac{s}{\sigma_{e e}}-\frac{s^{2} \beta^{2}}{a^{*} \sigma_{e e}^{2}}, \quad c^{*}=\frac{1}{2} a^{*-2} \sigma_{e e}^{-1} \sigma_{u u}^{-1}, \\
& a^{*}=r \sigma_{u u}^{-1}+\sigma_{x x}^{-1}+s \beta^{2} \sigma_{e e}^{-1}, \quad \mathbf{q}^{*^{\prime}}=\left[\mu_{x} v^{*}, \frac{s \mu_{x} \beta}{\sigma_{e e} \sigma_{x x} a^{*}}\right], \mathbf{Q}^{*}=\left[\begin{array}{cc}
v^{*} & \frac{s \beta}{a^{*} \sigma_{e e} \sigma_{x x}} \\
\frac{a^{*}-\sigma_{x x}^{-1}}{\sigma_{x x}}
\end{array}\right], \\
& \mathbf{t}^{*^{\prime}}=c^{*}\left[2 s \beta\left(r+s \beta^{2} \lambda^{-1}\right) \sigma_{x x}^{-1}, \quad-2 r s \beta \sigma_{u u}^{-1}, \quad \frac{2 s \beta}{\lambda}\left(a^{*}-s \beta^{2} \sigma_{e e}^{-1}\right)\right], \\
& \mathbf{T}=c^{*}\left[\begin{array}{ccc}
\sigma_{e e} \sigma_{u u}\left(\frac{a^{*}-\sigma_{x x}^{-1}}{\sigma_{x x}}\right)^{2} & \frac{r \lambda}{\sigma_{x x}^{2}} & \frac{s}{\lambda}\left(\frac{\beta}{\sigma_{x x}}\right)^{2} \\
\frac{r \lambda}{\sigma_{x x}^{2}} & r \lambda\left(a^{* 2}-\frac{2 a^{*}}{\sigma_{u u}}+\frac{r}{\sigma_{u u}^{2}}\right) & \frac{r s \beta^{2}}{\sigma_{e e} \sigma_{u u}} \\
\frac{s}{\lambda}\left(\frac{\beta}{\sigma_{x x}}\right)^{2} & \frac{r s \beta^{2}}{\sigma_{e e} \sigma_{u u}} & \frac{s}{\lambda}\left(a^{* 2}-\frac{2 a^{*} \beta^{2}}{\sigma_{e e}}+\frac{s \beta^{4}}{\sigma_{e e}^{2}}\right)
\end{array}\right] .
\end{aligned}
$$

Casos particulares

1) Fazendo $s=1$, obtemos a matriz de covariâncias assintóticas dos EMVs (2.27) para o caso da replicação balanceada só da variável explanatória; tratado na Seção 2.2. 2) Fazendo $s=r>1$, obtemos a matriz de covariâncias assintóticas dos EMVs para o caso tratado em Chan e Mak (1979a), ou seja, replicações balanceadas e iguais das variáveis explanatória e respostas. 
Caso não-balanceado

Quando $r_{t}$ varia com $t, t=1, \ldots, n$, temos

$$
f_{\mathbf{Z}_{t}}\left(\mathbf{z}_{t}\right)=(2 \pi)^{-\left(\frac{\left(r_{t}+1\right)}{2}\right)}\left(\operatorname{det} \mathbf{V}_{t}\right)^{-\frac{1}{2}} \exp \left(-\frac{1}{2}\left[\left(\mathbf{z}_{t}-\mathbf{m}_{t}\right)^{\prime} \mathbf{V}_{t}^{-1}\left(\mathbf{z}_{t}-\mathbf{m}_{t}\right)\right]\right)
$$

onde $\mathbf{m}_{t}$ e $\mathrm{V}_{t}$ estão definidos em 2.3.1.

Façamos

$$
l_{n}=\ln \prod_{t=1}^{n} f_{\mathbf{Z}_{t}}\left(\mathbf{z}_{t}\right)=\sum_{t=1}^{n} \ln f_{\mathbf{Z}_{t}}\left(\mathbf{z}_{t}\right)=\sum_{t=1}^{n} l_{t} .
$$

De (2.59) obtemos

$$
-E\left(\frac{\partial^{2} l_{n}}{\partial \psi \partial \omega}\right)=-E\left[\frac{\partial^{2}}{\partial \psi \partial \omega}\left(\sum_{t=1}^{n} l_{t}\right)\right]=-\sum_{t=1}^{n} E\left(\frac{\partial^{2} l_{t}}{\partial \psi \partial \omega}\right),
$$

onde $\psi$ e $\omega$ representam os parâmetros do modelo. De acordo com Dolby (1976), temos

$$
-E\left(\frac{\partial^{2} l_{t}}{\partial \psi \partial \omega}\right)=\left[\frac{1}{2} \operatorname{tr}\left(\mathbf{V}_{t}^{-1} \mathbf{V}_{t \psi} \mathbf{V}_{t}^{-1} \mathbf{V}_{t \phi}\right)+\mathbf{d}_{t \psi}^{\prime} \mathbf{V}_{t}^{-1} \mathbf{d}_{t \phi}\right]
$$

onde $\mathbf{d}_{t}$ e outras notações estão definidas na Seção 2.3.1.

Portanto, a matriz de covariâncias assintóticas dos EMVs no caso de replicações não-balanceadas da variável explanatória, por analogia ao caso balanceado, pode ser expressa como

$$
\mathbf{I}_{F_{t t}}=n\left[\begin{array}{ccc}
\bar{p}_{\beta \beta}+\mu_{x}^{2} \bar{v} & \overline{\mathbf{q}}_{12}^{\prime} & \overline{\mathbf{t}}^{\prime} \\
\overline{\mathbf{q}}_{21} & \overline{\mathbf{Q}}_{22} & \mathbf{0}_{23} \\
\overline{\mathbf{t}}_{31} & \mathbf{0}_{32} & \overline{\mathbf{T}}_{33}
\end{array}\right],
$$

onde:

$$
\begin{gathered}
\bar{p}_{\beta \beta}=2\left[\sigma_{x x}\left(\frac{\sum_{t=1}^{n} r_{t} a_{t} c_{t}}{n}\right)+\frac{2 \beta^{2}}{\lambda} \bar{c}\right], \quad \bar{v}=\frac{1}{\sigma_{e e}}-\frac{\beta^{2}}{\sigma_{e e}^{2}}\left(\frac{\sum_{t=1}^{n} \frac{1}{a_{t}}}{n}\right), \\
c_{t}=\frac{1}{2} a_{t}^{-2} \sigma_{u u}^{-1} \sigma_{e e}^{-1}, \quad \bar{c}=\frac{\sum_{t=1}^{n} c_{t}}{n}, \quad a_{t}=r_{t} \sigma_{u u}^{-1}+\sigma_{x x}^{-1}+\beta^{2} \sigma_{e e}^{-1}
\end{gathered}
$$




$$
\begin{gathered}
\overline{\mathbf{q}}^{\prime}=\left(\mu_{x} \bar{v}, \frac{\mu_{x} \beta}{\sigma_{e e} \sigma_{x x}}\left(\frac{\sum_{t=1}^{n} \frac{1}{a_{t}}}{n}\right)\right), \\
\overline{\mathbf{Q}}=\left(\begin{array}{cc}
\bar{v} & \frac{\beta}{\sigma_{e e} \sigma_{x x}}\left(\frac{\sum_{t=1}^{n} \frac{1}{a_{t}}}{n}\right) \\
\frac{\beta}{\sigma_{e e} \sigma_{x x}}\left(\frac{\left.\sum_{t=1}^{n} \frac{1}{a_{t}}\right)}{n}\right) & \frac{\bar{a}-\sigma_{x x}^{-1}}{\sigma_{x x}}
\end{array}\right), \quad \bar{a}=\frac{\sum_{t=1}^{n} a_{t}}{n} \\
\overline{\mathrm{e}}=\left(\begin{array}{c}
2 \beta\left(\frac{\sum_{t=1}^{n} r_{t} c_{t}}{n}+\beta^{2} \lambda^{-1} \bar{c}\right) \sigma_{x x}^{-1} \\
-2 \beta \sigma_{u u}^{-1}\left(\frac{\sum_{t=1}^{n} r_{t} c_{t}}{n}\right) \\
\frac{2 \beta}{\lambda}\left(\frac{\sum_{t=1}^{n} c_{t} a_{t}}{n}-\beta^{2} \sigma_{e e}^{-1} \bar{c}\right)
\end{array}\right)
\end{gathered}
$$$$
\mathbf{T}=\left[\begin{array}{ccc}
T_{11} & \frac{\lambda}{\sigma_{x x}^{2}}\left(\sum_{t=1}^{n} \frac{r_{t} c_{t}}{n}\right) & \frac{1}{\lambda}\left(\frac{\beta}{\sigma_{x x}}\right)^{2} \bar{c} \\
\frac{\lambda}{\sigma_{x x}^{2}}\left(\sum_{t=1}^{n} \frac{r_{t} c_{t}}{n}\right) & T_{22} & \frac{\beta^{2}}{\sigma_{e e} \sigma_{u u}} \sum_{t=1}^{n} \frac{r_{t} c_{t}}{n} \\
\frac{1}{\lambda}\left(\frac{\beta}{\sigma_{x x}}\right)^{2} \bar{c} & \frac{\beta^{2}}{\sigma_{e e} \sigma_{u u}} \sum_{t=1}^{n} \frac{r_{t} c_{t}}{n} & \frac{1}{\lambda}\left(\sum_{t=1}^{n} \frac{c_{t} a_{t}^{2}}{n}-\frac{2 \beta^{2}}{\sigma_{e e}} \sum_{t=1}^{n} \frac{a_{t} c_{t}}{n}+\frac{\beta^{4}}{\sigma_{e e}^{2}} \bar{c}\right)
\end{array}\right]
$$

com

$$
T_{11}=\frac{\sigma_{e e} \sigma_{u u}}{\sigma_{x x}^{2}}\left(\sum_{t=1}^{n} \frac{c_{t} a_{t}^{2}}{n}+\sigma_{x x}^{-2} \bar{c}-2 \sigma_{x x}^{-1} \sum_{t=1}^{n} \frac{c_{t} a_{t}}{n}\right)
$$

e

$$
T_{22}=\lambda\left(\sum_{t=1}^{n} \frac{r_{t} a_{t}^{2} c_{t}}{n}-\frac{2}{\sigma_{u u}} \sum_{t=1}^{n} \frac{r_{t} a_{t} c_{t}}{n}+\frac{1}{\sigma_{u u}^{2}} \sum_{t=1}^{n} \frac{r_{t}^{2} c_{t}}{n}\right)
$$

Vamos obter, agora, a variância assintótica de $\widehat{\beta}$. Fazendo a seguinte partição da matriz de Informação de Fisher multiplicada por $n^{-1}$ :

$$
n^{-1} \mathbf{I}_{F_{t t}}=\left[\begin{array}{cc:c}
\bar{p}_{\beta \beta}+\mu_{x}^{2} \bar{v} & \overline{\mathbf{q}}_{12}^{\prime} & \overline{\mathbf{t}}^{\prime} \\
\overline{\mathbf{q}}_{21} & \overline{\mathbf{Q}}_{22} & \mathbf{0}_{23} \\
\hdashline \overline{\mathbf{t}}_{31} & \mathbf{0}_{32} & \overline{\mathbf{T}}_{33}
\end{array}\right]
$$

e utilizando os teoremas A.5.2 e A.5.3 emMuirhead (1982), obtemos:

$$
\operatorname{AVAR}(\widehat{\beta})=\frac{1}{n} \frac{|\overline{\mathbf{T}}|}{|\overline{\mathbf{P}}|}
$$


onde $\operatorname{AVAR}(\widehat{\beta})$ denota variância assintótica de $\widehat{\beta}$ e

$$
\overline{\mathbf{P}}=\left[\begin{array}{cc}
\bar{p}_{\beta \beta} & \overline{\mathbf{t}}^{\prime} \\
\overline{\mathrm{t}} & \overline{\mathbf{T}}
\end{array}\right] .
$$

Observação: A matriz de Informação de Fisher dos EMV's no caso não-balanceado pode, também, ser obtida diretamente da definição, ou seja, usando

$$
-E\left(\frac{\partial^{2} l_{n}}{\partial \psi \partial \omega}\right)
$$

como elemento $\psi$ e $\omega$ da matriz, onde $\psi$ e $\omega$ representam os parâmetros do modelo. 


\section{Capítulo 3}

\section{Estimadores Alternativos}

\section{Envolvendo Replicações da Variável Explanatória}

\subsection{Introdução}

Este capítulo será dedicado à obtenção de estimadores alternativos do parâmetro de interesse $\beta$ do Modelo Estrutural Normal descrito pelas equações (1.1), (1.2), e suposições (1.4). O objetivo principal deste Capítulo é obter estimadores que possam competir (no que se refere à eficiência e à simplicidadade de cálculo), com os estimadores de máxima verossimilhança (que são os mais eficientes assintoticamente) obtidos no Capítulo 2, a partir de soluções fechadas no caso de número de replicações constante, ou algoritmo EM quando o número de replicações varia. Neste sentido, vários estimadores foram propostos no decorrer deste capítulo, numa seqüência que privilegiou aqueles que indicassem uma via que levasse aos estimadores sugeridos por Fuller (1987, Cap.2 e 3) e recomendados por Schafer e Purdy (1996). Justifica-se, assim, porque alguns estimadores só foram enunciados, sem um detalhamento mais completo.

Como já foi colocado anteriormente, quando todos os parâmetros $\left(\alpha, \beta, \mu_{x}, \sigma_{x x}\right.$, $\left.\sigma_{u u}, \sigma_{e e}\right)$ são desconhecidos o modelo é não-identificável; porém, se uma das suposições 
(S-1) a (S-3) (veja Seção 1.3.1) for válida, então o modelo torna-se identificável e a solução das equações de máxima verossimilhança tem uma expressão fechada e pode ser obtida pelo Método dos Momentos.

Consideremos, particularmente, que a suposição (S-1) seja válida, a saber, a variância do erro de mensuração, $\sigma_{u u}$, seja conhecida. Neste caso há expressões "fechadas" para os estimadores de máxima verossimilhança dos coeficientes de regressão (Fuller, 1987, Seção 2.2.1), obtidos resolvendo-se a equação:

$$
\left(\begin{array}{c}
\hat{\alpha} \\
\hat{\beta}
\end{array}\right)=\left(\begin{array}{cc}
n & \sum_{t=1}^{n} X_{t} \\
\sum_{t=1}^{n} X_{t} & \sum_{t=1}^{n} X_{t}^{2}-n \sigma_{u u}
\end{array}\right)^{-1}\left(\begin{array}{c}
\sum_{t=1}^{n} Y_{t} \\
\sum_{t=1}^{n} X_{t} Y_{t}
\end{array}\right)
$$

que levam a

$$
\hat{\beta}=\frac{m_{X Y}}{m_{X X}-\sigma_{u u}}, \quad \hat{\alpha}=\bar{Y}-\hat{\beta} \bar{X}
$$

em que

$$
m_{\mathrm{ZZ}}=\frac{\sum_{t=1}^{n}\left(\mathrm{Z}_{t}-\overline{\mathrm{Z}} \cdot\right)^{\prime}\left(\mathrm{Z}_{t}-\overline{\mathrm{Z}} .\right)}{n} \text { e } \mathrm{Z}_{t}=\left(X_{t}, Y_{t}\right)^{\prime} .
$$

Para obter estimadores alternativos para $\beta$, quando $\sigma_{u u}$ é desconhecido, pode-se inspirar na fórmula acima (3.1) usando uma idéia heurística. Em essência, ela pode ser colocada assim: se algum estimador de $\sigma_{u u}$, denotado por $\hat{\sigma}_{u u}$, estiver disponível, então a formulação (3.1) com $\sigma_{u u}$ substituído por $\hat{\sigma}_{u u}$ é um estimador natural com "correção por atenuação" de $\beta$. Estimadores dos outros parâmetros são derivados como na suposição de $\sigma_{u u}$ ser conhecido. Este estimador, obtido sob a suposição (S-1), desempenhará um papel central nesta pesquisa. Seguindo as sugestões de Fuller (1987, Cap.2 e 3) e as recomendações de Schafer e Purdy (1996), ele será "melhorado" ao incorporarmos de forma mais ampla as informações geradas pelas replicações. Somente os estimadores, concebidos sob esta perspectiva, serão comparados com os estimadores de máxima verossimilhança estudados no Capítulo 2.

Consideramos duas classes de estimadores. A primeira contendo os obtidos a partir das replicações balanceadas de $x_{t}$, neste caso os cálculos se simplificam e facilitam a obtenção da distribuição assintótica. Por outro lado são estimadores menos interessantes sob o ponto de vista prático, pois necessitam de replicações (iguais) de todas as 
observações. Já na segunda classe, com replicações não balanceadas, os estimadores adquirem maior relevância prática e são os mais enfatizados.

\subsection{Replicações Balanceadas}

Nesta seção obtemos os estimadores alternativos de $\beta$, a partir das estimações de $\sigma_{u u}$, $\lambda$ e $k_{x x}$ (veja Seção 1.3.2, desta tese), usando replicações constantes (balanceadas) da variável explanatória, ou seja, $r_{t}=r>1$, para $t=1, \ldots, n$. Para obter a distribuição assintótica destes estimadores, e também daqueles obtidos a partir de replicações nãobalanceadas, usamos um procedimento descrito de acordo com os três passos seguintes:

Passo 1. Construir uma seqüência de v.a. $\left\{U_{t}\right\}_{t \geq 1}$, tal que a expressão $(\sqrt{n})^{-1} \sum_{t=1}^{n} U_{t}$ seja função de termos componentes do estimador de $\beta$ em questão. Estes termos são as covariâncias amostrais $m_{Z Z}$ e as estatísticas suficientes geradas pelas replicações (Subseção 1.3.1).

Passo 2. Mostrar que a seqüência de v.a. $\left\{U_{t}\right\}_{t \geq 1}$ é constituída de v.a. iid no caso de replicações balanceadas ou satisfaz as condições do Teorema Central do Limite de Liapounov (James, 1996) no caso de replicações não balanceadas. Usar os teoremas do Limite Central (Durret 1991) e de Cramér-Wold (Sen e Singer, 1993) para obter a distribuição assintótica do vetor de termos citados no Passo 1, ou seja, os termos envolvidos no estimador de $\beta$.

Passo 3. Aplicar o método Delta para obter a distribuição assintótica do estimador sob estudo.

\subsubsection{Estimador de $\beta$ usando um EMV de $\sigma_{u u}$}

Dentro do contexto em que trabalhamos, a primeira tentativa de obter um estimador de $\sigma_{u u}$, será feita pelo método de máxima verossimilhança, a partir de replicações independentes e balanceadas da variável explanatória. 


\section{Obtenção do EMV de $\sigma_{u u}$}

Seja $\mathrm{X}=\left(\mathrm{X}_{1}^{\prime}, \ldots, \mathrm{X}_{n}^{\prime}\right)$ uma amostra independente, com $\mathrm{X}_{t}=\left(X_{t 1}, \ldots, X_{t r}\right)^{\prime}, t=$ $1, \ldots, n$ e $r>1$. Usando os procedimentos e suposições anteriores, o logaritmo da função do verossimilhança envolvendo apenas a informação de $\mathrm{X}$ pode ser escrito como

$$
\begin{aligned}
l_{u u}= & \text { cte }-\frac{n}{2} \ln \sigma_{x x}-\frac{n r}{2} \ln \sigma_{u u}-\frac{n}{2} \ln a_{u u}- \\
& -\frac{1}{2} \sum_{t=1}^{n}\left(\sigma_{u u}^{-1} \sum_{j=1}^{r}\left(X_{t j}-\mu_{x}\right)^{2}-a_{u u}^{-1}\left[\sigma_{u u}^{-1} \sum_{j=1}^{r}\left(X_{t j}-\mu_{x}\right)\right]^{2}\right),
\end{aligned}
$$

$\operatorname{com} a_{u u}=\sigma_{x x^{-1}}+r \sigma_{u u}^{-1}$.

Derivando (3.2) em relação aos parâmetros $\left(\mu_{x}, \sigma_{x x}, \sigma_{u u}\right)^{\prime}$ e igualando a zero obtemos as seguintes equações:

$$
\begin{aligned}
& \hat{\mu}_{x}=\frac{\sum_{t=1}^{n} \sum_{j=1}^{r} X_{t j}}{n r}, \\
& n a_{u u}^{2} \sigma_{x x}-n a_{u u}-\sum_{t=1}^{n}\left[\sigma_{u u}^{-1} \sum_{j=1}^{r}\left(X_{t j}-\bar{X}_{. .}\right]^{2}=0,\right. \\
& n r a_{u u}+a_{u u} \sigma_{u u}^{-1} \sum_{t=1}^{n} \sum_{j=1}^{r}\left(X_{t j}-\bar{X}_{. .}\right)^{2}-n r \sigma_{u u}^{-1}-n r^{2} \sigma_{u u}^{-2} \sigma_{x x}=0 .
\end{aligned}
$$

Das equações (3.3) a (3.4) obtemos o EMV de $\sigma_{u u}$,

$$
\hat{\sigma}_{u u}=\frac{\sum_{t=1}^{n} \sum_{j=1}^{r}\left(X_{t j}-\bar{X}_{t .}\right)^{2}}{n(r-1)} .
$$

Substituindo (3.6) em (3.1) obtemos um estimador de $\beta$ que será referido como $\hat{\beta}_{M}$, ou seja,

$$
\hat{\beta}_{M}=\frac{m_{X Y}}{m_{X X}-\hat{\sigma}_{u u}} .
$$

O teorema a seguir estabelece a distribuição assintótica de $\hat{\beta}_{M}$; para isso, entretanto precisaremos, antes, da definição (Sen e Singer, 1993): 
Definição 3.1 Sejam $\left\{X_{t}\right\}_{t \geq 1}$ uma seqüência de variáveis aleatórias e $\left\{b_{t}\right\}_{t \geq 1}$ seqüência de números reais (ou variáveis aleatórias), então diremos que

- $X_{t}=O_{p}\left(b_{t}\right)$ se para todo número real $\eta>0$ existirem um número real positivo $K=K(\eta)$ e um número inteiro positivo $t_{0}=t_{0}(K)$ tais que $P\left(\left|X_{t} / b_{t}\right| \geq K\right) \leq$ $\eta, \forall t \geq t_{0}$.

Teorema 3.1 Se as suposições adotadas até aqui forem satisfeitas então

1) $W_{t}=\sigma_{u u}^{-1} \sum_{j=1}^{r}\left(X_{t j}-\bar{X}_{t}\right)^{2} \sim \chi^{2}(r-1), \quad t=1, \ldots, n$.

2) $\sqrt{n}\left(\hat{\beta}_{M}-\beta\right) \stackrel{D}{\longrightarrow} \mathrm{N}\left(0, \gamma_{2}\right)$, quando $n \longrightarrow \infty$, onde

$$
\gamma_{2}=\sigma_{x x}^{-2}\left[\beta^{2} \sigma_{x x} \sigma_{u u}+\left(\sigma_{x x}+\sigma_{u u}\right) \sigma_{e e}+2 \beta^{2} \sigma_{u u}^{2}\left(\frac{r}{r-1}-\frac{2}{r}\right)\right] .
$$

\section{Prova:}

1) Seja $\mathbf{X}_{t}=\left(X_{t 1}, \ldots, X_{t r}\right)^{\prime}, t=1, \ldots, n$, o vetor de replicações de $x_{t}$, no qual $X_{t j}=$ $x_{t}+u_{t j}$, com $u_{t j} \sim \mathrm{N}\left(0, \sigma_{u u}\right)$. É fácil verificar que

$$
\frac{\sqrt{n} \bar{u}_{t .}}{\sigma_{u u}} \sim \mathrm{N}(0,1), \quad \text { e } \quad \sum_{j=1}^{r}\left(\frac{u_{t j}-\bar{u}_{t .}}{\sigma_{u u}}\right)^{2} \sim \chi^{2}(r-1),
$$

$\operatorname{com} \overline{u_{t .}}=\frac{\sum_{j=1}^{r} u_{t j}}{r}$. Mas,

$$
\begin{array}{r}
W_{t}=\sigma_{u u}^{-1} \sum_{t=1}^{r}\left(X_{t j}-\bar{X}_{t .}\right)^{2}=\sigma_{u u}^{-1} \sum_{t=1}^{r}\left[x_{t}-u_{t j}-\left(x_{t}-\bar{u}_{t .}\right)\right]^{2} \\
=\sum_{j=1}^{r}\left(\frac{u_{t j}-\bar{u}_{t .}}{\sigma_{u u}}\right)^{2} \sim \chi^{2}(r-1), \quad t=1, \ldots, n .
\end{array}
$$

2) Note que

$$
\widehat{\sigma_{u u n}}=\frac{\sum_{t=1}^{n} \sum_{j=1}^{r}\left(X_{t j}-\bar{X}_{t .}\right)^{2}}{n(r-1)}=\left(n^{-1}\right) \sum_{t=1}^{n} \frac{\sigma_{u u}}{r-1} W_{t}=\frac{\sum_{t=1}^{n} R_{t}}{n}
$$


onde

$$
R_{t}=\frac{\sum_{t=1}^{n}\left(X_{t j}-\bar{X}_{t .}\right)^{2}}{r-1}=\frac{\sigma_{u u}}{r-1} W_{t} \sim i i d\left[\frac{\sigma_{u u}}{r-1} \chi^{2}(r-1)\right] .
$$

Portanto, temos que $E\left(R_{t}\right)=\sigma_{u u}$, e $\operatorname{Var}\left(R_{t}\right)=\frac{2 \sigma_{u u}^{2}}{r-1}$. Logo, pelo teorema central do limite clássico temos

$$
\sqrt{n}\left(\widehat{\sigma_{u u n}}-\sigma_{u u}\right) \stackrel{\mathrm{D}}{\rightarrow} \mathrm{N}\left(0, \frac{2 \sigma_{u u}^{2}}{r-1}\right) .
$$

De acordo com o procedimento descrito na Seção 3.2, vamos construir abaixo a v.a. $U_{t}$. Temos que:

$$
\begin{aligned}
m_{X Y n}= & \left(n^{-1}\right) \sum_{t=1}^{n}\left(X_{t}-\bar{X}_{n}\right)\left(Y_{t}-\bar{Y}_{n}\right)=\left(n^{-1}\right) \sum_{t=1}^{n}\left(X_{t}-\mu_{x}\right)\left(Y_{t}-\mu_{y}\right) \\
& -\left(\bar{X}_{n}-\mu_{x}\right)\left(\bar{Y}_{n}-\mu_{y}\right)=\left(n^{-1}\right) \sum_{t=1}^{n}\left(X_{t}-\mu_{x}\right)\left(Y_{t}-\mu_{y}\right)+O_{p}\left(n^{-1}\right) \\
m_{X X n}= & \left(n^{-1}\right) \sum_{t=1}^{n}\left(X_{t}-\bar{X}_{n}\right)^{2}=\sum_{t=1}^{n}\left(X_{t}-\mu_{x}\right)^{2}+O_{p}\left(n^{-1}\right) \\
\widehat{\sigma_{u u}}= & \left(n^{-1}\right) \sum_{t=1}^{n} R_{t},
\end{aligned}
$$

em que $X_{t}=X_{t 1}=x_{t}+u_{t 1}$, para $t=1, \ldots, n$, ou seja, $X_{t}$ será a primeira replicação de $x_{t}$. Então, considerando $\mathbf{a}=\left(a_{1}, a_{2}, a_{3}\right)^{\prime} \in \mathbf{R}^{3}$, fixo, mas arbitrário, podemos escrever:

$$
\begin{aligned}
& \sqrt{n}\left[a_{1}\left(m_{X X n}-\sigma_{X X}\right)+a_{2}\left(m_{X Y n}-\sigma_{X Y}\right)+a_{3}\left(\hat{\sigma}_{u u_{n}}-\sigma_{u u}\right)\right] \\
& =(\sqrt{n})^{-1} \sum_{t=1}^{n}\left(a_{1}\left[\left(X_{t}-\mu_{n}\right)^{2}-\sigma_{X X}\right]+a_{2}\left[\left(X_{t}-\mu_{x}\right)\left(Y_{t}-\mu_{y}\right)-\sigma_{X Y}\right]\right. \\
& \left.+a_{3}\left(R_{t}-\sigma_{u u}\right)\right)+O_{p}\left(n^{-1}\right) \\
& =(\sqrt{n})^{-1} \sum_{t=1}^{n} U_{t}+O_{p}\left(n^{-1}\right),
\end{aligned}
$$

em que

$$
U_{t}=a_{1}\left[\left(X_{t}-\mu_{n}\right)^{2}-\sigma_{X X}\right]+a_{2}\left[\left(X_{t}-\mu_{x}\right)\left(Y_{t}-\mu_{y}\right)-\sigma_{X Y}\right]+a_{3}\left(R_{t}-\sigma_{u u}\right) .
$$


Sob as suposições do modelo, $\left\{U_{t}\right\}_{t \geq 1}$ é uma seqüência de v.a. independentes e, são também, identicamente distribuídas, pois $E\left(U_{t}\right)=0$ (por construção) e $E\left[U_{t}^{2}\right]=\mathbf{a}^{\prime} \Gamma \mathbf{a}$, em que $\Gamma=\operatorname{Cov}\left(Q_{1}, Q_{2}, Q_{3}\right)$, com $Q_{1}=\left(X_{t}-\mu_{n}\right)^{2}-\sigma_{X X}, Q_{2}=\left(X_{t}-\mu_{x}\right)\left(Y_{t}-\right.$ $\left.\mu_{y}\right)-\sigma_{X Y}$ e $Q_{3}=R_{t}-\sigma_{u u}$. Após algumas manipulações algébricas obtemos

$$
\Gamma=\left[\begin{array}{ccc}
2\left(\sigma_{X X}\right)^{2} & 2 \sigma_{X X} \sigma_{X Y} & \frac{2 \sigma_{u u}^{2}}{r} \\
2 \sigma_{X X} \sigma_{X Y} & \sigma_{X X} \sigma_{Y Y}+\left(\sigma_{X Y}\right)^{2} & 0 \\
\frac{2 \sigma_{u u}^{2}}{r} & 0 & \frac{2\left(\sigma_{u u}\right)^{2}}{r-1}
\end{array}\right],
$$

onde $\quad \sigma_{X X}=\sigma_{x x}+\sigma_{u u} \quad$ e $\quad \sigma_{X Y}=\beta \sigma_{x x}$.

Portanto, pelos teoremas do limite central clássico (James 1996) e do Cramér-Wold (Sen e Singer, 1993) segue-se que

$$
\sqrt{n}\left[\left(m_{X X_{n}}, m_{X Y_{n}}, \hat{\sigma}_{u u_{n}}\right)-\left(\sigma_{X X}, \beta \sigma_{x x}, \sigma_{u u}\right)\right] \stackrel{\mathrm{D}}{\longrightarrow} \mathrm{N}(0, \Gamma) .
$$

Como $\hat{\beta}_{M}=m_{X Y_{n}} /\left(m_{X X_{n}}-\hat{\sigma}_{u u_{n}}\right)$, podemos definir uma função $g: \mathbf{R}_{+}^{2} \times \mathbf{R} \rightarrow \mathbf{R}$, tal que $g\left(x_{1}, x_{2}, x_{3}\right)=x_{2} /\left(x_{1}-x_{3}\right)$, para todos $\left(x_{1}, x_{2}, x_{3}\right) \in \mathbf{R}_{+}^{2} \times \mathbf{R}$. Agora, aplicando o método Delta (Sen e Singer, 1993) obtemos

$$
\sqrt{n}\left(\widehat{\beta}_{M}-\beta\right) \stackrel{\mathrm{D}}{\longrightarrow} \mathrm{N}\left(0, \gamma^{2}\right),
$$

onde:

$$
\begin{aligned}
\gamma^{2}= & \sigma_{x x}^{-2}\left(2 \beta^{2} \sigma_{X X}^{2}-4 \beta \sigma_{X X} \sigma_{X Y}+\sigma_{X X} \sigma_{Y Y}+\sigma_{X Y}^{2}-\frac{4 \beta^{2} \sigma_{u u}^{2}}{r}\right. \\
& \left.+\frac{2 \beta^{2} \sigma_{u u}^{2}}{r-1}\right) \\
= & \sigma_{x x}^{-2}\left[\beta^{2} \sigma_{x x} \sigma_{u u}+\left(\sigma_{x x}+\sigma_{u u}\right) \sigma_{e e}+2 \beta^{2} \sigma_{u u}^{2}\left(\frac{r}{r-1}-\frac{2}{r}\right)\right],
\end{aligned}
$$

obtido após algumas manipulações algébricas.

\section{$\underline{\text { Eficiência relativa assintótica de } \hat{\beta}_{M} \text { e } \hat{\beta}_{M V}}$}

Denotemos por $\hat{\beta}_{M V}$ o estimador de máxima verossimilhança, dado em (2.23) obtido pelas replicações balanceadas de $x_{t}$. Sob as condições de regularidade provou-se 
na Seção 2.2 que

$$
\sqrt{n}\left(\widehat{\beta}_{M V}-\beta\right) \stackrel{\mathrm{D}}{\longrightarrow} \mathrm{N}\left(0, A V A R\left(\widehat{\beta}_{M V}\right)\right)
$$

onde

$$
A V A R\left(\widehat{\beta}_{M V}\right)=\frac{|\mathrm{T}|}{|\mathbf{P}|},
$$

com T e $\mathbf{P}$ definidos na Seção 2.2.3. Assim, de (3.8) e (3.9), segue-se que a eficiência assintótica de $\widehat{\beta}_{M V}$ e $\widehat{\beta}_{M}$ é

$$
\mathrm{e}_{\widehat{\beta}_{M V}, \widehat{\beta}_{M}}=\frac{\gamma^{2}}{A V A R\left(\widehat{\beta}_{M V}\right)} .
$$

\section{Avaliações Numéricas}

Apresentamos, a seguir, os resultados numéricos obtidos a partir de (3.10), para $\beta=\mu_{x}=\sigma_{x x}=\sigma_{u u}=\sigma_{e e}=1$ e $\alpha=5$.

Tabela 3.1 : Eficiência Assintótica

\begin{tabular}{|c|c|c|c|}
\hline número de replicações & $\mathrm{r}=2$ & $\mathrm{r}=5$ & $\mathrm{r}=10$ \\
\hline $\mathrm{e}_{\widehat{\beta}_{M V}, \widehat{\beta}_{M}}$ & 1,6667 & 3,1333 & 3,9455 \\
\hline
\end{tabular}

Pela Tabela 3.1 fica evidente a superioridade do EMV em relação ao estimador alternativo, e a tendência é que a eficiência aumente com o número de replicações $r$.

\subsubsection{Estimadores de $\beta$ baseados em EMV de $\lambda$ e $k_{x x}$}

Podemos obter outros estimadores ao substituirmos nas soluções "fechadas" das equações de verossimilhança, as suposições que resolvem a questão de identificabilidade, por estimadores obtidos através de replicações balanceadas da variável explanatória.

Sob a suposição da razão de variâncias conhecida, $\lambda$, temos o seguinte estimador de $\beta$, que denotaremos por $\widehat{\beta}_{\hat{\lambda}}$, (veja Fuller, 1987, eq.(1.37)):

$$
\widehat{\beta}_{\lambda}=\frac{m_{Y Y}-\lambda \cdot m_{X X}+\left[\left(m_{Y Y}-\lambda \cdot m_{X X}\right)^{2}+4 \lambda^{2} m_{X Y}^{2}\right]^{\frac{1}{2}}}{2 m_{X Y}} .
$$


Um estimador de $\lambda$ foi obtido na subseção 2.2 .2 , equação (2.25), a saber:

$$
\begin{aligned}
\hat{\lambda} & =\frac{\left(\widehat{\beta}_{1}^{\prime}\right)^{2} S_{X Y}-\widehat{\beta}_{1}^{\prime} S_{Y Y}}{r\left(S_{X Y}-\widehat{\beta}_{1}^{\prime} S_{X X}\right)}= \\
& =\left(\frac{r-1}{r}\right)\left[\frac{(r-1) S_{X Y}^{2}-S_{Y Y}\left(r S_{X X}-t_{X X}\right)}{\left(S_{X X}-t_{X X}\right)\left(r S_{X X}-t_{X X}\right)}\right] .
\end{aligned}
$$

Substituindo $\lambda$ pelo estimador acima na equação (3.11) obteremos um novo estimador para $\beta$, denotado por $\widehat{\beta}_{\hat{\lambda}}$.

Supondo o fator de atenuação $k_{x x}$ conhecido, temos o seguinte estimador de $\beta \quad$ (veja Fuller, 1987):

$$
\widehat{\beta}_{k_{x x}}=\left(k_{x x}\right)^{-1} \widehat{\beta}_{M Q O}=\left(\frac{\sigma_{x x}+\sigma_{u u}}{\sigma_{x x}}\right) \widehat{\beta}_{M Q O},
$$

onde $\beta_{M Q O}$ é o estimador de mínimos quadrados ordinários (Fuller 1987). Um estimador para $k_{x x}$ pode se obtido das equações (3.3) a (3.4) como:

$$
\widehat{k_{x x}}=\frac{\widehat{\sigma_{u u} n}}{\widehat{\sigma_{x x} n}+\widehat{\sigma_{u u}}},
$$

em que $\widehat{\sigma}_{u u}$ é dada pela equação (3.6) e, de (3.4) e (3.5), podemos obter:

$$
\widehat{\sigma_{x x} n}=\frac{\sum_{t=1}^{n}\left(X_{t .}-\bar{X}_{. .}\right)^{2}}{n}-\frac{\widehat{\sigma_{u u} n}}{n} .
$$

Substituindo $k_{x x}$ pelo seu estimador (3.13) obteremos um novo estimador para $\beta$ que denotaremos por $\widehat{\beta}_{k_{x x}}^{*}$.

\section{- Observações:}

Não tentamos obter a distribuição assintótica destes estimadores, pois eles não desempenharão papeis relevantes no que segue. No entanto, elas podem ser obtidas de maneira similar à dedução feita para $\hat{\beta}_{M}$.

Alguns melhoramentos podem ser sugeridos como, por exemplo, introduzindo as seguintes modificações: 
(a) Substituir $X_{t 1}$ por $X_{t}$, onde $X_{t .}=\frac{\sum_{j=1}^{r} X_{t j}}{r}$;

(b) Multiplicar $\widehat{\sigma_{u u}}$ por $\frac{1}{r}$ (como conseqüência da primeira modificação).

Com estas modificações os estimadores $\hat{\beta}_{M}$ (3.7) e $\widehat{\beta}_{k_{x \boldsymbol{x}}}$ (3.12) coincidem com o EMV denotado por $\hat{\beta}_{M V}$ em (2.23).

\section{Avaliações Numéricas}

Apresentamos, a seguir, os resultados de simulações comparando o comportamento dos estimadores alternativos de $\beta$ com o EMV, para 2000 amostras com duplas replicações simuladas pelo programa escrito na linguagem $\mathrm{Ox}$, sob o modelo (2.1) e suposições (2.2).

Para a comparação do desempenho dos estimadores, calcularam-se as seguintes medidas:

(i) Vício relativo médio observado, definido por

$E M R=\sum_{i=1}^{2000}\left(\hat{\beta}_{i}-\beta\right) / \beta$, onde $\beta_{i}$ é o estimador em questão.

(ii) Erro quadrático médio relativo observado, definido por

$$
E Q M R=\sum_{i=1}^{2000}\left(\hat{\beta}_{i}-\beta\right)^{2} / \beta^{2} .
$$

Os valores dos parâmetros utilizados para as simulações foram :

(a) $\beta=\mu_{x}=\sigma_{x x}=\sigma_{u u}=\sigma_{e e}=\alpha=1$ e $\mathrm{n}=100$.

Tabela 3.2 : Vícios e EQMR's empíricos

\begin{tabular}{|l|c|c|c|c|c|}
\hline & $\hat{\beta}_{M V}$ & $\hat{\beta}_{M}$ & $\hat{\beta}_{k x x}$ & $\hat{\beta}_{\hat{\lambda}}$ & $\hat{\beta}_{\lambda C}$ \\
\hline Vício Relativo (Vícios $/ \beta)$ & 0,0251 & 0,0596 & 0,03056 & 0,04735 & 0,3222 \\
\hline EQM Relativo (EQM $\left./ \beta^{2}\right)$ & 0,03475 & 0,09185 & 0,04406 & 0,0413 & 0,1427 \\
\hline
\end{tabular}

(b) $\mu_{x}=68 ; \alpha=63 ; \beta=0,5 ; \sigma_{x x}=240 ; \sigma_{e e}=50$ e dois valores para $\sigma_{u u}$ : 60 e 240 . 
Tabela 3.3 : Vícios e EQMR's empíricos

\begin{tabular}{|l|c|c|c|c|c|}
\hline & $\hat{\beta}_{M V}$ & $\hat{\beta}_{M}$ & $\hat{\beta}_{k x x}$ & $\hat{\beta}_{\hat{\lambda}}$ & $\hat{\beta}_{\lambda C}$ \\
\hline Vício Relativo (Vícios/ $\beta$ ) & 0,0265 & 0,0711 & 0,0331 & $-0,0407$ & $-0,1265$ \\
EQM Relativo $\left(\mathrm{EQM} / \beta^{2}\right)$ & 0,0384 & 0,1256 & 0,0494 & 0,2298 & 0,0869 \\
\hline
\end{tabular}

Aqui, $\hat{\beta}_{\lambda C}$ é obtido ao substituir $X_{t 1}$ por $X_{t}$, sendo $X_{t}=\frac{\sum_{j=1}^{r} X_{t j}}{r}$, no estimador $\hat{\beta}_{\lambda}$; os outros já foram definidos anteriormente. Pelas Tabelas 3.2 e 3.3 fica evidente a superioridade do EMV em relação aos estimadores alternativos, quando comparados com as duas medidas acima enunciadas. No entanto para n relativamente pequeno esta superioridade não se manifesta como podemos verificar pelos resultados apresentados na Tabela 3.4 para $n=30$ e os mesmos valores paramétricos utilizados na tabela a seguir.

Tabela 3.4 : Vícios e EQMR's empíricos

\begin{tabular}{|l|c|c|c|c|c|}
\hline & \multicolumn{1}{|c|}{$\hat{\beta}_{M V}$} & $\hat{\beta}_{M}$ & $\hat{\beta}_{k x x}$ & $\hat{\beta}_{\hat{\lambda}}$ & $\hat{\beta}_{\lambda C}$ \\
\hline Vício Relativo (Vícios/ $\beta$ ) & 0,02420 & 0,1284 & 0,2420 & $-0,0760$ & $-0,1283$ \\
EQM Relativo (EQM/ $\left./ \beta^{2}\right)$ & 19,21 & 63,28 & 17,54 & 38,09 & 6,38 \\
\hline
\end{tabular}

Isto ocorre porque a condição para $\lambda>0$ não está se verificando.

\subsection{Replicações Não-Balanceadas}

\subsubsection{Introdução}

Nesta seção examinamos os estimadores alternativos de $\beta$, usando replicações nãobalanceadas da variável explanatória. Utilizamos o mesmo processo descrito na Seção 3.2 para obter as distribuições assintóticas dos estimadores. Embora, aqui, $\left\{U_{t}\right\}_{t \geq 1}$ seja uma seqüência de v.as. que são independentes mas não identicamente distribuídas há a possibilidade de usar o teorema central do limite de Lyapunov (Durret 1991). Consideraremos dois estimadores para $\sigma_{u u}$. O primeiro procura seguir uma estrutura semelhante à do EMV de $\sigma_{u u}(3.6)$, ou seja, 


$$
S_{u u 1 n}=\frac{\sum_{t=1}^{n}\left(\frac{\sum_{j=1}^{r_{t}}\left(X_{t j}-\bar{X}_{t .}\right)^{2}}{r_{t}-I_{r_{t}}(1)}\right)}{\sum_{t=1}^{n} I_{r_{t}}(1)}, \quad \text { para } \quad r_{t} \geq 1
$$

onde

$$
I_{r_{t}}(k)=\left\{\begin{array}{c}
1, \text { se } r_{t} \geq k \\
0, \quad \text { cc }
\end{array}\right.
$$

e $\bar{X}_{t .}=\frac{\sum_{j=1}^{r_{t}} X_{t j}}{r_{t}}$.

O segundo estimador de $\sigma_{u u}$ proposto é baseado na análise de variância para estimar os componentes da variância. Segundo Fuller (1995) este estimador, que apresentaremos a seguir, não é o mais eficiente, embora seja mais fácil de construir. Seja então,

$$
S_{u u 2 n}=\frac{\sum_{t=1}^{n}\left(\sum_{j=1}^{r_{t}}\left(X_{t j}-\bar{X}_{t .}\right)^{2}\right)}{\sum_{t=1}^{n}\left(r_{t}-1\right)}, \quad \text { para } \quad r_{t} \geq 1 .
$$

\subsubsection{Estimadores de $\beta$ considerando $S_{u u 1 n}$}

Sob a suposição de $r_{t}>1$ para pelo menos um $t, t=1, \ldots, n$, verificamos no capítulo anterior que o EMV de $\beta$ não tem uma forma analítica fechada, mas a sua matriz de informação é análoga ao caso balanceado e foi obtida na Seção 2.4.4; portanto, para efeito de comparação de eficiência assintótica com os estimadores aqui propostos, isto é suficiente.

A semelhança da estrutura do estimador $S_{u u 1 n}$ com à do EMV de $\sigma_{u u}$ (3.6) facilita a obtenção da distribuição assintótica dos estimadores de $\beta$ gerados a partir dele, pois podemos seguir um roteiro pré-traçado na Seção precedente. Portanto a motivação da análise deste estimador, nesta perspectiva, tem um caráter nitidamente mais ditático que utilitário; ou seja, a sua inclusão já se justifica, por se constituir, pelo contraste com os anteriores, num liame necessário, no encadeamento dos estimadores pesquisados. No entanto, isto não implica que os estimadores gerados por ele sejam menos eficientes que 
os gerados pelo $S_{u u 2 n}$. Com o estimador $S_{u u 1 n}$, podemos considerar os dois seguintes estimadores de $\beta$ equivalentes ao (3.7):

\section{(1) Estimador $\hat{\beta}_{N B 1}$.}

Substitua em (3.7), $\hat{\sigma}_{u u}$ por (3.14), ou seja

$$
\hat{\beta}_{N B 1}=\frac{m_{X Y}}{m_{X X}-S_{u u 1 n}} .
$$

O teorema a seguir estabelece a distribuição assintótica de $\hat{\beta}_{N B 1}$.

Teorema 3.2 Se as suposições adotadas até aqui forem satisfeitas e se existir o

$$
\lim _{n \rightarrow \infty} \sum_{t=1}^{n} \frac{I_{r_{t}}(1)}{n}=\nu_{1} \text {, então }
$$

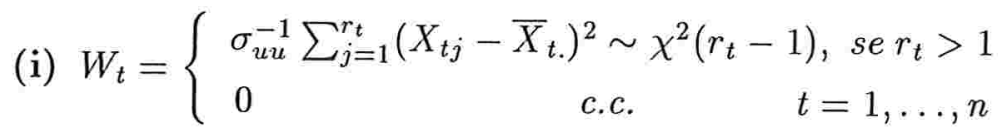

(ii) $\sqrt{n}\left(\hat{\beta}_{N B 1}-\beta\right) \stackrel{D}{\longrightarrow} \mathrm{N}\left(0, \gamma_{1}^{2}\right)$, quando $n \longrightarrow \infty$, em que

$$
\gamma_{1}^{2}=\sigma_{x x}^{-2}\left[2 \beta^{2} \sigma_{u u}^{2}+\left(\sigma_{x x}+\sigma_{u u}\right) \sigma_{e e}+\beta^{2} \sigma_{x x} \sigma_{u u}-4 \beta^{2} \nu_{3} \sigma_{u u}^{2}+2 \beta^{2} \nu_{1} \nu_{2} \sigma_{u u}^{2}\right] .
$$

\section{Prova:}

(i) Pode ser demonstrado de modo análogo ao item correspondente no

Teorema 2.1

(ii) Se $r_{t}>1$, do item anterior temos

$$
R_{1 t}=\frac{\sum_{j=1}^{r_{t}}\left(X_{t j}-\bar{X}_{t .}\right)^{2}}{r_{t}-1}=\frac{\sigma_{u u}}{r_{t}-1} W_{t} \sim i i d\left[\frac{\sigma_{u u}}{r_{t}-1} \chi^{2}\left(r_{t}-1\right)\right] .
$$

Portanto, temos que $E\left(R_{1 t}\right)=\sigma_{u u}$ e $\operatorname{Var}\left(R_{1 t}\right)=2 \sigma_{u u}^{2} /\left(r_{t}-1\right) \leq 2 \sigma_{u u}^{2} /(2-1)=$ $2 \sigma_{u u}$, pois $r_{t}>1$. Supondo que $0<\sigma_{u u}<\infty$, temos que $0<\operatorname{Var}\left(R_{1 t}\right)<\infty$.

A seqüência $\left(R_{1 t}\right)_{t \geq 1}$ é independente, mas não identicamente distribuída, entretanto satisfaz as condições do teorema central do limite de Liapounov, como demonstraremos a seguir, para $\delta=1$. Para tanto é suficiente demonstrar que

$$
\lim _{n \rightarrow \infty} \frac{1}{s_{n}^{2+\delta}} \sum_{t=1}^{n} E\left|R_{1 t}-I_{r_{t}}(1) \sigma_{u u}\right|^{2+\delta}=0,
$$


onde $s_{n}^{2}=\sum_{t=1}^{n} \operatorname{Var}\left(R_{1 t}\right)$.

Temos também que, se $r_{t}>1$ :

$$
\begin{aligned}
E\left|R_{1 t}-\sigma_{u u}\right|^{3} \leq E\left|R_{1 t}+\sigma_{u u}\right|^{3} & =E\left(R_{1 t}+\sigma_{u u}\right)^{3} \\
& =E\left(R_{1 t}^{3}+3 R_{1 t}^{2} \sigma_{u u}+3 R_{1 t} \sigma_{u u}^{2}+\sigma_{u u}^{3}\right) .
\end{aligned}
$$

Após algumas manipulações algébricas, obtemos

$$
E\left(R_{1 t}^{3}\right)=E\left(\frac{\sum_{j=1}^{r_{t}} u_{t j}^{2}-r_{t} \bar{u}_{t .}^{2}}{r_{t}-1}\right)^{3}=\left(\frac{1}{r_{t}-1}\right)^{3}\left[9\left(r_{t}-1\right)^{2}+6\left(r_{t}-1\right)\right] \sigma_{u u}^{3},
$$

pois

$$
E\left(3 \sigma_{u u} R_{1 t}^{2}\right)=3 \sigma_{u u}^{3}+\left(\frac{6}{r_{t}-1}\right) \sigma_{u u}^{3} .
$$

Substituindo (3.20) e (3.21) em (3.19) temos

$$
E\left(R_{1 t}+\sigma_{u u}\right)^{3}=\left(\frac{15}{r_{t}-1}+\frac{6}{\left(r_{t}-1\right)^{2}}+7\right) \sigma_{u u}^{3} \leq 28 \sigma_{u u}^{3} .
$$

Por outro lado, temos

$$
s_{n}^{2}=\sum_{t=1}^{n} \operatorname{Var}\left(R_{1 t}\right)=\sum_{t=1}^{n}\left(\frac{2 \sigma_{u u}^{2} I_{r_{t}}(1)}{r_{t}-I_{r_{t}}(1)}\right),
$$

$\log 0$

$$
s_{n}=\sqrt{2} \sigma_{u u} \sqrt{\sum_{t=1}^{n} \frac{I_{r_{t}}(1)}{r_{t}-I_{r_{t}}(1)}} \geq \sqrt{2} \sigma_{u u} \sqrt{\frac{n \nu_{n}}{r^{*}}}
$$

onde $r^{*}=\max \left(r_{t}\right)<\infty, t=1, \ldots, n$ e $\nu_{n}=\frac{\sum_{t=1}^{n} I_{r_{t}}(1)}{n}$. Admitamos a existência de $\lim _{n \rightarrow \infty} \nu_{n}=\nu \leq 1$.

De (3.22) e (3.23) temos (3.18), ou seja,

$$
\lim _{n \rightarrow \infty} \frac{1}{s_{n}^{2+\delta}} \sum_{t=1}^{n} E\left|R_{1 t}-I_{r_{t}}(1) \sigma_{u u}\right|^{2+\delta}=0 .
$$


Conseqüentemente, o teorema central do limite de Liapounov garante que

$$
\frac{\frac{\sum_{t=1}^{n} R_{1 t}}{n}-E\left(\frac{\sum_{t=1}^{n} R_{1 t}}{n}\right)}{\sqrt{\operatorname{Var}\left(\frac{\sum_{t=1}^{n} R_{1 t}}{n}\right)}} \stackrel{\mathrm{D}}{\longrightarrow} \mathrm{N}(0,1) .
$$

Agora, fazendo $X_{t}=X_{t 1}, t=1, \ldots, n$ e usando o mesmo procedimento descrito na Seção 3.2 vamos construir a v.a. $U_{1 t}$. Temos que

$$
\begin{aligned}
& \sqrt{n}\left[a_{1}\left(m_{X_{1} X_{1 n}}-\sigma_{X X}\right)+a_{2}\left(m_{X_{1} Y_{n}}-\sigma_{X Y}\right)\right. \\
& \left.+a_{3}\left(S_{u u 1 n}-I_{r_{t}}(1) \sigma_{u u}\right)\right] \\
= & (\sqrt{n})^{-1} \sum_{t=1}^{n}\left\{a_{1}\left[\left(X_{t 1}-\mu_{n}\right)^{2}-\sigma_{X X}\right]+a_{2}\left[\left(X_{t 1}-\mu_{x}\right)\left(Y_{t}-\mu_{y}\right)-\sigma_{X Y}\right]\right. \\
& \left.+a_{3} \nu_{1 n}\left(R_{1 t}-I_{r_{t}}(1) \sigma_{u u}\right)\right\}+O_{p}\left(n^{-1}\right) \\
= & (\sqrt{n})^{-1} \sum_{t=1}^{n} U_{1 t}+O_{p}\left(n^{-1}\right),
\end{aligned}
$$

onde

$$
U_{1 t}=a_{1}\left[\left(X_{t 1}-\mu_{x}\right)^{2}-\sigma_{X X}\right]+a_{2}\left[\left(X_{t 1}-\mu_{x}\right)\left(Y_{t}-\mu_{y}\right)-\sigma_{X Y}\right]+a_{3} \nu_{1 n}\left(R_{1 t}-I_{r_{t}}(1) \sigma_{u u}\right)
$$$$
\text { e } \nu_{1 n}=n / \sum_{t=1}^{n} I_{r_{t}}(1) \text { para } t=1, \ldots, n \text {. }
$$

Por construção $\left\{U_{1 t}\right\}$ é uma seqüência de v.a independentes com $E\left(U_{1 t}\right)=0$, e pode-se verificar que:

$$
\begin{aligned}
\operatorname{Var}\left(U_{1 t}\right) & =2 a_{1}^{2} \sigma_{X X}^{2}+4 a_{1} a_{2} \sigma_{X X} \sigma_{X Y}+a_{1} a_{3} \nu_{1 n}\left(\frac{4 I_{r_{t}}(1)}{r_{t}}\right) \sigma_{u u}^{2} \\
& +\left[a_{2}^{2}\left(\sigma_{X X} \sigma_{Y Y}+\sigma_{X Y}^{2}\right)\right]+2 a_{3}^{2} \nu_{1 n}^{2}\left(\frac{I_{r_{t}}(1) \sigma_{u u}^{2}}{r_{t}-I_{r_{t}}(1)}\right)=\mathbf{a}^{\prime} \Gamma_{1 t} \mathbf{a},
\end{aligned}
$$

em que $\sigma_{X X}=\sigma_{x x}+\sigma_{u u}, \sigma_{X Y}=\beta \sigma_{x x}, \sigma_{Y Y}=\beta^{2} \sigma_{x x}+\sigma_{e e}$ e 


$$
\Gamma_{1 t}=\left[\begin{array}{ccc}
2 \sigma_{X X}^{2} & 2 \sigma_{X X} \sigma_{X Y} & \nu_{1 n}\left(\frac{2 I_{r_{t}}(1)}{r_{t}}\right) \sigma_{u u}^{2} \\
2 \sigma_{X X} \sigma_{X Y} & \sigma_{X X} \sigma_{X Y}+\sigma_{X Y}^{2} & 0 \\
\nu_{1 n}\left(\frac{2 I_{r_{t}}(1)}{r_{t}}\right) \sigma_{u u}^{2} & 0 & \nu_{1 n}^{2}\left(\frac{2 I_{r_{t}}(1)}{r_{t}-I_{r_{t}}(1)}\right) \sigma_{u u}^{2}
\end{array}\right] .
$$

Portanto, $\left(U_{1 t}\right)_{t \geq 1}$ é uma seqüência de variáveis aleatórias independentes porém não identicamente distribuídas. Todavia, ela satisfaz as condições de Liapounov como verificamos a seguir. Após exaustivas manipulações algébricas (veja Apêndice C.4) obtemos:

$$
\begin{array}{r}
E\left|U_{1 t}-E\left(U_{1 t}\right)\right|^{3}=E\left|U_{1 t}\right|^{3} \leq 15 a_{m}^{3}\left[\sigma_{X X}+2 \sigma_{X Y}+(2+|\beta|) \sqrt{\sigma_{u u} \sigma_{x x}}\right. \\
\left.+\sqrt{\sigma_{e e}}\left(\sqrt{\sigma_{x x}}+\sigma_{u u}\right)\right]^{3}
\end{array}
$$

onde $a_{m}=\max \left(\left|a_{1}\right|,\left|a_{2}\right|,\left|a_{3}\right|\right)$.

Agora, admitindo existência dos limites

$$
\begin{gathered}
\lim _{n \rightarrow \infty} \nu_{1 n}=\lim _{n \rightarrow \infty}\left(\frac{n}{\sum_{t=1}^{n} \frac{1}{r_{t}}}\right)=\nu_{1}, \quad 1 \leq \nu_{1}<\infty, \\
\lim _{n \rightarrow 0}\left(\frac{\sum_{t=1}^{n}\left(\frac{I_{r_{t}}(1)}{r_{t}-I_{r_{t}}(1)}\right)}{\sum_{t=1}^{n} I_{r_{t}}(1)}\right)=\nu_{2} \leq 1, \\
\lim _{n \rightarrow 0}\left(\frac{\sum_{t=1}^{n}\left(\frac{I_{r_{t}}(1)}{r_{t}}\right)}{\sum_{t=1}^{n} I_{r_{t}}(1)}\right)=\nu_{3}<1
\end{gathered}
$$

e usando (3.24) e (C.113) vem que

$$
\frac{1}{\left(s_{n}\right)^{3}} \sum_{z=1}^{n} E\left|U_{1 t}\right|^{3} \rightarrow 0 .
$$

Conseqüentemente, pelo teorema central do limite de Liapounov temos que

$$
\frac{\sum_{t=1}^{n} U_{1 t}-E\left(\sum_{t=1}^{n} U_{1 t}\right)}{\sqrt{\operatorname{Var}\left(\sum_{t=1}^{n} U_{1 t}\right)}} \stackrel{\mathrm{D}}{\longrightarrow} N(0,1) .
$$


Agora, vamos mostrar que a seqüência $\left\{U_{t}\right\}_{t \geq 1}$ satisfaz as condições do Lema Gleser-Ruppert (Gleser 1981 e Gallo 1982), (veja o enunciado no Apêndice B.2.2).

1. Mostramos que $\left\{U_{1 t}\right\}_{t \geq 1}$ é uma seqüência de v.as mutuamente independentes, e satisfaz as condições do teorema central do limite de Liapounov.

2. Mostramos (veja Apêndice C.3) que $E\left(U_{1 t}\right)^{4}<\infty$ (condição imposta por Ruppert (Gallo 1982) para validar o resultado de Gleser (1981)).

3. Note que

$$
\frac{\sum_{t=1}^{n} \operatorname{Var}\left(U_{1 t}\right)}{n}=\mathbf{a}^{\prime}\left[\begin{array}{ccc}
2 \sigma_{X X}^{2} & 2 \sigma_{X X} \sigma_{X Y} & 2 \sigma_{u u}^{2}\left(\begin{array}{cc}
\sum_{t=1}^{n} \frac{I_{r_{t}}(1)}{r_{t}} \\
\sum_{t=1}^{n} I_{r_{t}}(1)
\end{array}\right) \\
2 \sigma_{X X} \sigma_{X Y} & \sigma_{X X} \sigma_{X Y}+\sigma_{X Y}^{2} & 0 \\
2 \sigma_{u u}^{2}\left(\frac{\sum_{t=1}^{n} \frac{I_{r_{t}}(1)}{r_{t}}}{\sum_{t=1}^{n} I_{r_{t}}(1)}\right) & 0 & 2 \sigma_{u u}^{2} \nu_{1 n}\left(\frac{\sum_{t=1}^{n} \frac{I_{r_{t}}(1)}{r_{t}-I_{r_{t}}(1)}}{\sum_{t=1}^{n} I_{r_{t}}(1)}\right)
\end{array}\right] \mathbf{a},
$$

e considerando as suposições (3.26), (3.27) e (3.28), temos que

$$
\Gamma_{1}=\lim _{n \rightarrow \infty}\left(\frac{\sum_{t=1}^{n} \operatorname{Var}\left(U_{1 t}\right)}{n}\right)=\mathbf{a}^{\prime}\left[\begin{array}{ccc}
2 \sigma_{X X}^{2} & 2 \sigma_{X X} \sigma_{X Y} & 2 \sigma_{u u}^{2} \nu_{3} \\
2 \sigma_{X X} \sigma_{X Y} & \sigma_{X X} \sigma_{X Y}+\sigma_{X Y}^{2} & 0 \\
2 \sigma_{u u}^{2} \nu_{3} & 0 & 2 \sigma_{u u}^{2} \nu_{1} \nu_{2}
\end{array}\right] \mathbf{a} .
$$

Agora, pelo teorema de Cramér-Wold e o lema de Gleser-Ruppert (Gallo, 1982), temos que

$$
\sqrt{n}\left[\left(m_{X_{1} X_{1 n}}, m_{X_{1} Y_{n}}, S_{u u 1 n}\right)^{\prime}-\left(\sigma_{X X}, \beta_{1} \sigma_{x x}, \sigma_{u u}\right)^{\prime}\right] \stackrel{\mathrm{D}}{\longrightarrow} N\left(0, \Gamma_{1}\right),
$$

em que $\Gamma_{1}=\lim _{n \rightarrow \infty} \frac{\sum_{t=1}^{n} \Gamma_{1 t}}{n}, \operatorname{com} \quad \Gamma_{1 t}=\operatorname{Cov}\left(W_{11}, W_{12}, W_{13 t}\right), \quad W_{11}=\left[\left(X_{t 1}-\right.\right.$ $\left.\left.\mu_{x}\right)^{2}-\sigma_{X X}\right], W_{12}=\left[\left(X_{t 1}-\mu_{x}\right)\left(Y_{t}-\mu_{y}\right)-\sigma_{X X}\right]$ e $W_{13 t}=\left[R_{1 t}-\sigma_{u u}\right]$.

Como $\hat{\beta}_{N B 1}=m_{X_{1} Y_{n}} /\left(m_{X_{1} X_{1 n}}-S_{u u 1 n}\right)$, definamos $g(\mathbf{x}): \mathbf{R}^{+} \times \mathbf{R} \times \mathbf{R}^{+} \rightarrow \mathbf{R}$ onde

$$
g\left(x_{1}, x_{2}, x_{3}\right)=\frac{x_{2}}{x_{1}-x_{3}} \quad \text { e } \quad \dot{g}=\left(\frac{\partial g}{\partial x_{1}}, \frac{\partial g}{\partial x_{2}}, \frac{\partial g}{\partial x_{3}}\right)^{\prime}
$$


de modo que

$$
\dot{g}\left(x_{1}, x_{2}, x_{3}\right)=\left(\frac{-x_{2}}{\left(x_{1}-x_{3}\right)^{2}}, \frac{1}{x_{1}-x_{3}}, \frac{x_{2}}{\left(x_{1}-x_{3}\right)^{2}}\right)^{\prime}
$$

e como $\theta_{1}=\left(\sigma_{X X}, \sigma_{X Y}, \sigma_{u u}\right)^{\prime}$, temos

$$
\dot{g}\left(\theta_{1}\right)=\left(\frac{-\sigma_{X Y}}{\sigma_{x x}^{2}}, \frac{1}{\sigma_{x x}}, \frac{\sigma_{X Y}}{\sigma_{x x}^{2}},\right)^{\prime}=\sigma_{x x}^{-1}(-\beta, 1, \beta)^{\prime} .
$$

Portanto, aplicando o método Delta, obtemos

$$
\sqrt{n}\left(\hat{\beta}_{N B 1}-\beta\right) \stackrel{\mathrm{D}}{\longrightarrow} N\left(0, \gamma_{1}^{2}\right)
$$

em que $\gamma_{1}^{2}=\left(\dot{g}\left(\theta_{1}\right)\right)^{\prime} \Gamma_{1} \dot{g}\left(\theta_{1}\right)$. Após algumas manipulações algébricas, obtemos

$$
\begin{aligned}
\gamma_{1}^{2}= & \sigma_{x x}^{-2}\left(2 \beta^{2} \sigma_{X X}^{2}-4 \beta \sigma_{X X} \sigma_{X Y}+\sigma_{X X} \sigma_{Y Y}+\sigma_{X X Y}^{2}-4 \beta^{2} \nu_{3} \sigma_{u u}^{2}\right. \\
& \left.+2 \beta^{2} \nu_{1} \nu_{2} \sigma_{u u}^{2}\right) \\
= & \sigma_{x x}^{-2}\left[2 \beta^{2} \sigma_{u u}^{2}+\left(\sigma_{x x}+\sigma_{u u}\right) \sigma_{e e}+\beta^{2} \sigma_{x x} \sigma_{u u}-4 \beta^{2} \nu_{3} \sigma_{u u}^{2}+2 \beta^{2} \nu_{1} \nu_{2} \sigma_{u u}^{2}\right] .
\end{aligned}
$$

\section{(2) Estimador $\hat{\beta}_{N B 2}$}

O estimador em $\hat{\beta}_{N B 1}$ (3.17) pode ser apropriadamente modificado ao substituirmos a variável $X_{t_{1}}$ pela média $\bar{X}_{t}$. de um grupo de $r_{t}$ observações; isto, em geral, melhora a eficiência assintótica do estimador de $\beta$. Ao trabalhar com $\bar{X}_{t}$, o erro $u_{1 t}$ de $X_{t 1}$ passará a ser $\bar{u}_{t .}=\frac{\sum_{j=1}^{r_{t}} u_{t j}}{r_{t}}$, tal que, $\operatorname{Var}\left(\bar{u}_{t}.\right)=\frac{\sigma_{u u}}{r_{t}}=\sigma_{u u_{t}}$. Portanto, quando há replicações da variável explanatória a modificação sugerida por Fuller (1987, Seção 2.5 ), supondo $\sigma_{u u}$ conhecido, pode ser colocada como

$$
\left(\begin{array}{c}
\widehat{\alpha^{*}} \\
\widehat{\beta}^{*}
\end{array}\right)=\left(\begin{array}{cc}
n & \sum_{t=1}^{n} \bar{X}_{t} \\
\sum_{t=1}^{n} \bar{X}_{t} & \sum_{t=1}^{n}\left(\bar{X}_{t}^{2}-\sigma_{u u_{t}}\right)
\end{array}\right)^{-1}\left(\begin{array}{c}
\sum_{t=1}^{n} Y_{t} \\
\sum_{t=1}^{n} \bar{X}_{t} Y_{t}
\end{array}\right),
$$

ou seja,

$$
\widehat{\beta}^{*}=\frac{m_{\bar{X} Y}}{m_{\bar{X} \bar{X}}-\kappa_{n} \sigma_{u u}}, \quad \widehat{\alpha}^{*}=\bar{Y}-\widehat{\beta}^{*} \bar{X}_{. .}^{*}
$$


em que

$$
\begin{aligned}
m_{\bar{X} Y_{n}} & =\frac{\sum_{t=1}^{n}\left(\bar{X}_{t .}-\bar{X}_{. .}^{*}\right)\left(Y_{t}-\bar{Y} .\right)}{n}, \quad m_{\bar{X} \bar{X}_{n}}=\frac{\sum_{t=1}^{n}\left(\bar{X}_{t .}-\bar{X}_{. .}^{*}\right)^{2}}{n}, \\
\bar{X}_{. .}^{*}=\frac{\sum_{t=1}^{n} \bar{X}_{t .}}{n} & \text { e } \frac{\sum_{t=1}^{n} \sigma_{u u t}}{n}=\kappa_{n} \sigma_{u u} \quad \operatorname{com} \quad \kappa_{n}=\frac{\sum_{t=1}^{n} \frac{1}{r_{t}}}{n} .
\end{aligned}
$$

Portanto, podemos propor o segundo estimador de $\beta$ usando $S_{u u 1 n}$ como estimador de $\sigma_{u u}$ :

$$
\widehat{\beta}_{N B 2}=\frac{m_{\bar{X} Y}}{m_{\bar{X} \bar{X}}-\kappa_{n} S_{u u 1 n}} .
$$

A seguir apresentamos alguns cálculos necessários para obtenção da distribuição assintótica de (3.32), ou mais especificamente, para construir a v.a. $U_{2 t}$, conforme o procedimento já adotado. Seja então:

$$
\begin{gathered}
\sum_{t=1}^{n}\left(\bar{X}_{t .}-\bar{X}_{. .}^{*}\right)^{2}=\sum_{t=1}^{n}\left(\bar{X}_{t .}-\mu_{x}\right)^{2}-n\left(\bar{X}_{. .}^{*}-\mu_{x}\right)^{2}, \\
\sum_{t=1}^{n}\left(\bar{X}_{t .}-\bar{X}_{. .}\right)\left(Y_{t}-\bar{Y}_{.}\right)=\sum_{t=1}^{n}\left(\bar{X}_{t .}-\mu_{x}\right)\left(Y_{t}-\mu_{y}\right)-n\left(\bar{X}_{. .}^{*}-\mu_{x}\right)\left(\bar{Y}_{.}-\mu_{y}\right)^{2}, \\
\operatorname{Var}\left(\bar{X}_{t .}\right)=\sigma_{x x}+\frac{\sigma_{u u}}{r_{t}}=\sigma_{X X_{t}} \quad e \quad \sigma_{\bar{X}_{X_{n}}}=\frac{\sum_{t=1}^{n} \sigma_{X X_{t}}}{n}
\end{gathered}
$$

de modo que

$$
\begin{gathered}
\bar{X}_{t .}=\frac{\sum_{j=1}^{r_{t}} X_{t j}}{r_{t}} \sim N\left(\mu_{x} ; \sigma_{X X_{t}}\right), \\
Y_{t} \sim N\left(\mu_{y} ; \sigma_{Y Y}\right),
\end{gathered}
$$

e

$$
\frac{\sqrt{n}\left(\bar{X}_{. .}^{*}-\mu_{x}\right)}{\sqrt{\frac{\sum_{t=1}^{n} \sigma_{X X_{t}}}{n}}} \sim N(0,1) .
$$

De (3.36) e (3.37), temos

$$
\begin{gathered}
\bar{X}_{t .}-\mu_{x}=O_{p}\left(n^{-\frac{1}{2}}\right), \\
Y_{t}-\mu_{y}=O_{p}\left(n^{-\frac{1}{2}}\right) .
\end{gathered}
$$


De (3.33), (3.34), (3.38) e (3.39) e, analogamente, à demonstração anterior, podemos escrever:

$$
\begin{aligned}
& \sqrt{n}\left[a_{1}\left(m_{\bar{X} \bar{X}_{n}}-\sigma_{\bar{X} \bar{X}_{n}}\right)+a_{2}\left(m_{\bar{X} Y_{n}}-\sigma_{\bar{X} Y}\right)\right. \\
& \left.+a_{3}\left(\frac{\sum_{t=1}^{n} \frac{1}{r_{t}}}{n}\right)\left(S_{u u 1 n}-\sigma_{u u}\right)\right] \\
= & (\sqrt{n})^{-1} \sum_{t=1}^{n}\left(a_{1}\left[\left(\bar{X}_{t .}-\mu_{x}\right)^{2}-\sigma_{X X_{t}}\right]+a_{2}\left[\left(\bar{X}_{t .}-\mu_{x}\right)\left(Y_{t}-\mu_{y}\right)-\sigma_{\bar{X} Y}\right]\right. \\
& \left.+a_{3} \nu_{4 n}\left(R_{1 t}-I_{r_{t}}(1) \sigma_{u u}\right)\right)+O_{p}\left(n^{-1}\right) \\
= & (\sqrt{n})^{-1} \sum_{t=1}^{n} U_{2 t}+O_{p}\left(n^{-1}\right),
\end{aligned}
$$

onde $\nu_{4 n}=\frac{\sum_{t=1}^{n} \frac{1}{r_{t}}}{\sum_{t=1}^{n} I_{r_{t}}(1)}$. Portanto, temos

$$
\begin{aligned}
U_{2 t}= & a_{1}\left[\left(\bar{X}_{t .}-\mu_{x}\right)^{2}-\sigma_{X X_{t}}\right]+a_{2}\left[\left(\bar{X}_{t .}-\mu_{x}\right)\left(Y_{t}-\mu_{y}\right)-\sigma_{\bar{X} Y}\right] \\
& +a_{3} \nu_{4 n}\left[R_{1 t}-\sigma_{u u} I_{r_{t}}(1)\right]
\end{aligned}
$$

em que $\mathbf{a}=\left(a_{1}, a_{2}, a_{3}\right)^{\prime}$ é um vetor fixo e arbitrário de $\mathbb{R}^{3}$.

Por construção $\left\{U_{2 t}\right\}_{t \geq 1}$ é uma seqüência de v.as. independentes com média $E\left(U_{2 t}\right)=0$. Pode-se mostrar (Apêndice C.5) que:

$$
\begin{aligned}
\operatorname{Var}\left(U_{2 t}\right)=E\left(U_{2 t}^{2}\right)= & 2 a_{1}^{2} \sigma_{\bar{X} \bar{X}_{t}}^{2}+4 a_{1} a_{2} \sigma_{\bar{X} \bar{X}_{t}} \sigma_{\bar{X} Y}+a_{2}^{2}\left(\sigma_{\bar{X} \bar{X}_{t}} \sigma_{Y Y}+\sigma_{\bar{X} Y}^{2}\right) \\
& +a_{3}^{2} \nu_{4 n}^{2}\left(\frac{2 \sigma_{u u}^{2} I_{r_{t}}(1)}{r_{t}-I_{r_{t}}(1)}\right)=\mathbf{a}^{\prime} \Gamma_{2 t} \mathbf{a}<\infty,
\end{aligned}
$$

em que (veja os cálculos no Apêndice C.5):

$$
\begin{aligned}
\Gamma_{2 t} & =\operatorname{Cov}\left\{W_{21 t}, W_{22 t}, W_{23 t}\right\}, \\
\text { onde } & \\
W_{21 t} & =\left[\left(\bar{X}_{t .}-\mu_{x}\right)^{2}-\sigma_{X X_{t}}\right], \\
W_{22 t} & =\left[\left(\bar{X}_{t .}-\mu_{x}\right)\left(Y_{t}-\mu_{y}\right)-\sigma_{\bar{X} Y}\right], \\
W_{23 t} & =\nu_{4 n}\left[R_{1 t}-\sigma_{u u} I_{r_{t}}(1)\right],
\end{aligned}
$$


e

$$
\Gamma_{2 \mathrm{t}}=\left[\begin{array}{ccc}
2 \sigma_{X X_{t}}^{2} & 2 \sigma_{X X_{t}} \sigma_{\bar{X} Y} & 0 \\
2 \sigma_{X X_{t}} \sigma_{\bar{X} Y} & \sigma_{X X_{t}} \sigma_{Y Y}+\sigma_{\bar{X} Y}^{2} & 0 \\
0 & 0 & 2 \sigma_{u u}^{2} \nu_{4 n}^{2}\left(\frac{I_{r_{t}}(1)}{r_{t}-I_{r_{t}}(1)}\right)
\end{array}\right] .
$$

Entretanto, a condição de Liapounov está satisfeita, pois (veja Apêndice C.4):

$$
\begin{aligned}
E\left|U_{2 t}\right|^{3} \leq & 15\left\{2\left|a_{1}\right| \sigma_{\bar{X} \bar{X}_{t}}+2\left|a_{1}\right| \sigma_{\bar{X} Y}+\left(2\left|a_{1}\right|+\left|a_{2}\right||\beta|\right) \sqrt{\frac{\sigma_{x x} \sigma_{u u}}{r_{t}}}\right. \\
& \left.+\left|a_{2}\right| \sqrt{\sigma_{e e}}\left(\sqrt{\sigma_{x x}}+\sqrt{\frac{\sigma_{u u}}{r_{t}}}\right)+\left|a_{3}\right| \sigma_{u u}\left(\sqrt{\frac{r_{t}+1}{r_{t}-1}}+1\right)^{2}\right\}^{3}<\infty .
\end{aligned}
$$

Conseqüentemente, de (3.41) e (C.112) e admitindo-se a existência dos limites

$$
\begin{gathered}
\lim _{n \rightarrow \infty} \nu_{4 n}=\nu_{4}, \\
\lim _{n \rightarrow \infty} \nu_{5 n}=\lim _{n \rightarrow \infty}\left(\frac{\sum_{t=1}^{n} \frac{I_{r_{t}}(1)}{r_{t}-I_{r_{t}}(1)}}{n}\right)=\nu_{5}<1, \\
\lim _{n \rightarrow \infty} \kappa_{n}=\kappa<1, \\
\lim _{n \rightarrow \infty} \frac{\sum_{t=1}^{n} \frac{1}{r_{t}^{2}}}{n}=\nu_{6}<1,
\end{gathered}
$$

temos que

$$
\frac{1}{\left(\sqrt{\sum_{t=1}^{n} \operatorname{Var}\left(U_{2 t}\right)}\right)^{3}} \sum_{t=1}^{n} E\left|U_{2 t}\right|^{3} \rightarrow 0 .
$$

Logo, pelo teorema central de Liapounov,

$$
\frac{\sum_{t=1}^{n} U_{2 t}}{\sqrt{\frac{\operatorname{Var(U_{2t})}}{n}}} \stackrel{\mathrm{D}}{\longrightarrow} N(0,1) \text {. }
$$


Além disso, esta seqüência também satisfaz as condições do lema de Gleser-Ruppert, pois:

(i) $E\left[U_{2 t}^{4}\right]<\infty \quad$ (veja Apêndice C.3).

(ii) Note que:

$$
\begin{aligned}
& \frac{\sum_{t=1}^{n} \Gamma_{2 \mathrm{t}}}{n}=\left[\begin{array}{ccc}
2 \sigma_{\bar{X} \bar{X}_{n}}^{* 2} & 2 \sigma_{\bar{X} \bar{X}_{n}} \sigma_{\bar{X} Y} & 0 \\
2 \sigma_{\bar{X} \bar{X}_{n}} \sigma_{\bar{X} Y} & \sigma_{\bar{X} \bar{X}_{n}} \sigma_{Y Y}+\sigma_{\bar{X} Y}^{2} & 0 \\
0 & 0 & 2 \sigma_{u u}^{2} \nu_{4 n}^{2} \nu_{5 n}
\end{array}\right] \\
& \text { onde } \quad \sigma_{\bar{X} \bar{X}_{n}}=\sigma_{x x}+\kappa_{n} \sigma_{u u} \quad \text { e } \quad \sigma_{\bar{X} \bar{X}_{n}}^{* 2}=\frac{\sum_{t=1}^{n} \sigma_{X X_{t}}^{2}}{n}=\frac{\sum_{t=1}^{n}\left(\sigma_{x x}+\frac{\sigma_{u u}}{r_{t}}\right)}{n} \text {. }
\end{aligned}
$$

Portanto, considerando as condições acima e as suposições (3.44), (3.45) e (3.46), temos, pelo lema de Gleser-Ruppert, que

$$
\sqrt{n}\left(\frac{\sum_{t=1}^{n} U_{2 t}}{n}\right) \stackrel{\mathrm{D}}{\longrightarrow} N\left(0, \mathbf{a}^{\prime} \Gamma_{2} \mathbf{a}\right)
$$

onde

$$
\mathbf{a}^{\prime} \boldsymbol{\Gamma}_{2} \mathbf{a}=\lim _{n \rightarrow \infty} \frac{V\left(\sum_{t=1}^{n} U_{2 t}\right)}{n}=\lim _{n \rightarrow \infty} \frac{\sum_{t=1}^{n} \mathbf{a}^{\prime} \Gamma_{2} \mathbf{a}}{n},
$$

ou, equivalentemente,

$$
\Gamma_{2}=\lim _{n \rightarrow \infty} \frac{\sum_{t=1}^{n} \Gamma_{2 t}}{n}=\left[\begin{array}{ccc}
2 \sigma_{\bar{X} \bar{X}}^{* 2} & 2 \sigma_{X Y} \sigma_{\bar{X} \bar{X}} & 0 \\
2 \sigma_{X Y} \sigma_{\bar{X} \bar{X}} & \sigma_{Y Y} \sigma_{\bar{X} \bar{X}}+\sigma_{X Y}^{2} & 0 \\
0 & 0 & 2 \sigma_{u u}^{2} \nu_{4}^{2} \nu_{5}
\end{array}\right]
$$

com

$$
\sigma_{\bar{X} \bar{X}}^{* 2}=\sigma_{x x}^{2}+\nu_{6} \sigma_{u u}^{2}+2 \kappa \sigma_{x x} \sigma_{u u}, \quad \sigma_{\bar{X} \bar{X}}=\sigma_{x x}+\kappa \sigma_{u u}
$$

Agora, pelos teoremas central do limite e do Cramér-Wold, temos:

$$
\sqrt{n}\left[\left(m_{\bar{X} \bar{X}_{n}}, m_{\bar{X} Y_{n}}, \kappa_{n} S_{u u_{1 n}}\right)^{\prime}-\left(\sigma_{\bar{X} \bar{X}_{n}}, \beta \sigma_{x x}, \kappa_{n} \sigma_{u u}\right)^{\prime}\right] \stackrel{\mathrm{D}}{\longrightarrow} N\left(\mathbf{0}, \Gamma_{2}\right) .
$$


A distribuição assintótica de $\widehat{\beta}_{N B 2}$ pode ser obtida pelo método delta (Sen and Singer 1993), analogamente, ao caso anterior,

$$
\sqrt{n}\left(\hat{\beta}_{N B 2}-\beta\right) \stackrel{\mathrm{D}}{\longrightarrow} N\left(0, \gamma_{2}^{2}\right)
$$

em que $\gamma_{2}^{2}=\left(\dot{g}\left(\theta_{2}\right)\right)^{\prime} \Gamma_{2} \dot{g}\left(\theta_{2}\right)$, com $\theta_{2}=\left(\sigma_{\bar{X} \bar{X}_{n}}, \beta \sigma_{x x}, \kappa_{n} \sigma_{u u}\right)^{\prime}$. Após algumas manipulações algébricas, obtemos

$$
\begin{aligned}
\gamma_{2}^{2} & =\sigma_{x x}^{-2}\left(2 \beta^{2} \sigma_{\bar{X} \bar{X}}^{* 2}-4 \beta \sigma_{\bar{X} \bar{X}} \sigma_{X Y}+\sigma_{\bar{X} \bar{X}} \sigma_{Y Y}+\sigma_{X Y}^{2}+2 \beta^{2} \nu_{4}^{2} \nu_{5} \sigma_{u u}^{2}\right) \\
& =\sigma_{x x}^{-2}\left[\kappa \beta^{2} \sigma_{x x} \sigma_{u u}+\left(\sigma_{x x}+\kappa \sigma_{u u}\right) \sigma_{e e}+2 \beta^{2} \sigma_{u u}^{2}\left(\nu_{6}+\nu_{4}^{2} \nu_{5}\right)\right]
\end{aligned}
$$

\subsubsection{Estimador de $\beta$ considerando o estimador $S_{u u 2 n}$}

Um estimador de $\sigma_{u u}$ baseado em replicações não balanceadas proposto por Fuller (1987, Seção 3.1.2), é (3.16), ou seja

$$
S_{u u 2 n}=\frac{\sum_{t=1}^{n}\left(\sum_{j=1}^{r_{t}}\left(X_{t j}-\bar{X}_{t .}\right)^{2}\right)}{\sum_{t=1}^{n}\left(r_{t}-1\right)}, \quad \text { para } \quad 1 \leq r_{t}<\infty .
$$

Como já dissemos anteriormente, este estimador segundo Fuller (1995), não é o mais eficiente, embora seja mais fácil de construir. Substituindo $\sigma_{u u}$ em $\hat{\beta}^{*}$ (3.30), pelo estimador em (3.47), vamos obter o seguinte estimador para $\beta$

$$
\hat{\beta}_{N B 3}=\frac{m_{\bar{X} Y}}{m_{\bar{X} \bar{X}}-\kappa_{n} S_{u u 2 n}},
$$

ou, equivalentemente,

$$
\widehat{\beta}_{N B 3}=\frac{n^{-1} \sum_{t=1}^{n}\left(\bar{X}_{t .}-\bar{X}_{. .}^{*}\right)\left(Y_{t}-\bar{Y} .\right)}{n^{-1} \sum_{t=1}^{n}\left(\bar{X}_{t .}-\bar{X}_{. .}^{*}\right)^{2}-\left(\frac{\sum_{t=1}^{n} \frac{1}{r_{t}}}{\sum_{t=1}^{n}\left(r_{t}-1\right)}\right)\left(\frac{\sum_{t=1}^{n} \sum_{j=1}^{r_{t}}\left(\bar{X}_{t j}-\bar{X}_{t .}\right)^{2}}{n}\right)} .
$$

Suposição adotada

$$
\lim _{n \rightarrow \infty} \frac{\sum_{t=1}^{n} \frac{1}{r_{t}}}{\sum_{t=1}^{n}\left(r_{t}-1\right)}=\lim _{n \rightarrow \infty} \nu_{n}=\nu<\infty,
$$


com $r_{t}<\infty$ e $r_{t}>1$ para pelo menos um $t, t=1, \ldots, n$.

A seguir apresentamos alguns cálculos para a obtenção da distribuição assintótica de $\widehat{\beta}_{N B 3}$ dado em (3.49). Primeiro,

$$
\begin{gathered}
E\left(S_{u u 2 n}\right)=\frac{\sum_{t=1}^{n} E\left(\sum_{j=1}^{r_{t}}\left(X_{t j}-\bar{X}_{t}\right)^{2}\right)}{\sum_{t=1}^{n}\left(r_{t}-1\right)}=\frac{\sum_{t=1}^{n}\left(r_{t}-1\right) \sigma_{u u}}{\sum_{t=1}^{n}\left(r_{t}-1\right)}=\sigma_{u u} . \\
\text { Definindo } R_{3 t}=\sum_{j=1}^{r_{t}}\left(X_{t j}-\bar{X}_{t .}\right)^{2}, \text { obtemos } S_{u u 2 n}=\frac{\sum_{t=1}^{n} R_{3 t}}{\sum_{t=1}^{n}\left(r_{t}-1\right)}
\end{gathered}
$$

e, ainda,

$$
\begin{aligned}
E\left(m_{\bar{X} \bar{X}_{n}}\right) & =E\left(n^{-1} \sum_{t=1}^{n}\left(\bar{X}_{t .}-\bar{X}_{. .}^{*}\right)^{2}\right)=\left(\frac{n-1}{n}\right) \frac{\sum_{t=1}^{n} \sigma_{\bar{X} \bar{X}_{t}}}{n} \\
& =\left(\frac{n-1}{n}\right) \sigma_{\bar{X}_{X_{n}}}=\left(\frac{n-1}{n}\right)\left(\sigma_{x x}+\kappa_{n} \sigma_{u u}\right), \\
E\left(m_{\bar{X} Y_{n}}\right) & =E\left(n^{-1} \sum_{t=1}^{n}\left(\bar{X}_{t .}-\bar{X}_{. .}^{*}\right)\left(Y_{t}-Y .\right)\right. \\
& =\left(\frac{n-1}{n}\right) \beta \sigma_{x x}=\left(\frac{n-1}{n}\right) \sigma_{X Y}=\left(\frac{n-1}{n}\right) \sigma_{\bar{X} Y} .
\end{aligned}
$$

Agora, de (3.33), (3.34), (3.38)e (3.39), podemos escrever

$$
\begin{aligned}
& \sqrt{n}\left[a_{1}\left(m_{\bar{X} \bar{X}_{n}}-\sigma_{\bar{X} \bar{X}_{n}}\right)+a_{2}\left(m_{\bar{X} Y_{n}}-\sigma_{X Y}\right)+a_{3}\left(\kappa_{n} S_{u u 2 n}\right.\right. \\
& \left.\left.-\left(\frac{\sum_{t=1}^{n}\left(r_{t}-1\right)}{\sum_{t=1}^{n}\left(r_{t}-1\right)}\right) \kappa_{n} \sigma_{u u}\right)\right] \\
& =(\sqrt{n})^{-1} \sum_{t=1}^{n}\left\{\left(a_{1}\left[\left(\bar{X}_{t .}-\mu_{x}\right)^{2}-\sigma_{X X_{t}}\right]+a_{2}\left[\left(\bar{X}_{t .}-\mu_{x}\right)\left(Y_{t}-\mu_{y}\right)-\sigma_{X Y}\right]\right.\right. \\
& \left.+a_{3}\left(\frac{\sum_{t=1}^{n} \frac{1}{r_{t}}}{\sum_{t=1}^{n}\left(r_{t}-1\right)}\right)\left[R_{3 t}-\left(r_{t}-1\right) \sigma_{u u}\right]\right\}+O_{p}\left(n^{-1}\right) \\
& =(\sqrt{n})^{-1} \sum_{t=1}^{n} U_{3 t}+O_{p}\left(n^{-1}\right) .
\end{aligned}
$$

Assim, temos que

$$
\begin{aligned}
U_{3 t}= & a_{1}\left[\left(\bar{X}_{t .}-\mu_{x}\right)^{2}-\sigma_{X X_{t}}\right]+a_{2}\left[\left(\bar{X}_{t .}-\mu_{x}\right)\left(Y_{t}-\mu_{y}\right)-\sigma_{\bar{X} Y}\right] \\
& +a_{3} \nu_{n}\left[R_{3 t}-\left(r_{t}-1\right) \sigma_{u u}\right] .
\end{aligned}
$$


Pelos argumentos já apresentados, $\left\{U_{3 t}\right\}_{t \geq 1}$ é uma seqüência de v.as. independentes com média zero e variância dada por (veja os cálculos no Apêndice C.5)

$$
\begin{aligned}
\operatorname{Var}\left(U_{3 t}\right)= & 2 a_{1}^{2} \sigma_{X X_{t}}^{2}+4 a_{1} a_{2} \sigma_{X X_{t}} \sigma_{\bar{X} Y}+a_{2}^{2} \sigma_{X X_{t}} \sigma_{Y Y}+a_{2}^{2} \sigma_{\bar{X} Y}^{2} \\
& +2 a_{3}^{2} \nu_{n}{ }^{2}\left(r_{t}-1\right) \sigma_{u u}<\infty
\end{aligned}
$$

Logo $\left\{U_{3 t}\right\}_{t \geq 1}$ não é uma seqüência de v.as. iid, entretanto, como nos casos anteriores, satisfaz as condições do teorema central do limite de Liapounov e do Lema de GleserRuppert, como verificamos a seguir (veja os cálculos nos Apêndices C.3 e C.4).

$$
\begin{aligned}
E\left|U_{3 t}\right|^{3} \leq & 15 a_{m}^{3}\left\{2 \sigma_{X X_{t}}+2 \sigma_{\bar{X} Y}+2\left(r_{t}+1\right) \sigma_{u u}+(2+\beta) \sqrt{\frac{\sigma_{x x} \sigma_{u u}}{r_{t}}}\right. \\
& \left.+\sigma_{e e}\left(\sqrt{\sigma_{x x}}+\sqrt{\frac{\sigma_{u u}}{r_{t}}} I_{r_{t}}(1)\right)\right\}^{3}<\infty .
\end{aligned}
$$

Definamos KE3 e KV3, tais que

$$
\begin{aligned}
& 15 a_{m}^{3}\left\{2 \sigma_{X X_{t}}+2 \sigma_{\bar{X} Y}+2\left(r_{t}+1\right) \sigma_{u u}+(2+\beta) \sqrt{\frac{\sigma_{x x} \sigma_{u u}}{r_{t}}}\right. \\
& \left.+\sigma_{e e}\left(\sqrt{\sigma_{x x}}+\sqrt{\frac{\sigma_{u u}}{r_{t}}} I_{r_{t}}(1)\right)\right\}^{3} \leq K E 3<\infty \\
& \mathrm{e} \\
& 0<K V 3 \leq \operatorname{Var}\left(U_{3 t}\right)=2 a_{1}^{2} \sigma_{X X_{t}}^{2}+4 a_{1} a_{2} \sigma_{X X_{t}} \sigma_{\bar{X} Y}+a_{2}^{2} \sigma_{X X_{t}} \sigma_{Y Y}+a_{2}^{2} \sigma_{\bar{X} Y}^{2} \\
& +2 a_{3}^{2} \nu_{n}^{2}\left(r_{t}-1\right) \sigma_{u u}<\infty, \quad \text { para } t=1, \ldots, n .
\end{aligned}
$$

Portanto, de (3.59) e (3.60), e as suposiçôes (3.46) e (3.50) temos

$$
\frac{\sum_{t=1}^{n} E\left|U_{3 t}\right|^{3}}{\left[\sqrt{\sum_{t=1}^{n} \operatorname{Var}\left(U_{3 t}\right)}\right]^{3}} \leq \frac{n K E 3}{(\sqrt{n(K V 3)})^{3}}=\left(\frac{K E 3}{\sqrt{K V 3}}\right)(n)^{-\frac{1}{2}} \rightarrow 0 .
$$

Fazendo

$$
\begin{aligned}
W_{31_{t}} & =\left(\bar{X}_{t .}-\mu_{x}\right)^{2}-\sigma_{X X_{t}}, \\
W_{32_{t}} & =\left(\bar{X}_{t .}-\mu_{x}\right)\left(Y_{t}-\mu_{y}\right)-\sigma_{\bar{X} Y}, \\
\mathrm{e} & \\
W_{33_{t}} & =\hat{\nu}\left(R_{t}^{*}-\left(r_{t}-1\right) \sigma_{u u}\right)
\end{aligned}
$$


temos, após algumas manipulações algébricas (veja Apêndice C.5 ), que a matriz de covariâncias de $\left(W_{31_{t}}, W_{32_{t}}, W_{33_{t}}\right)^{\prime}$ pode ser escrita como:

$$
\Gamma_{3 \mathrm{t}}=\left[\begin{array}{ccc}
2\left(\sigma_{X X_{t}}\right)^{2} & 2 \sigma_{X X_{t}} \sigma_{\bar{X} Y} & 0 \\
2 \sigma_{X X_{t}} \sigma_{\bar{X} Y} & \sigma_{X X_{t}} \sigma_{Y Y}+\sigma_{\bar{X} Y}^{2} & 0 \\
0 & 0 & 2\left(\nu_{n}\right)^{2}\left(r_{t}-1\right) \sigma_{u u}^{2}
\end{array}\right]
$$

Com as suposições (3.45), (3.46) e (3.50), temos que:

$$
\lim _{n \rightarrow \infty} \frac{\sum_{t=1}^{n} \Gamma_{3 t}}{n}=\Gamma_{3}
$$

onde

$$
\Gamma_{3}=\left[\begin{array}{ccc}
2\left(\sigma_{x x}^{2}+2 \kappa \sigma_{u u} \sigma_{x x}+\nu_{6} \sigma_{u u}^{2}\right) & 2 \sigma_{X Y}\left(\sigma_{x x}+\kappa \sigma_{u u}\right) & 0 \\
2 \sigma_{X Y}\left(\sigma_{x x}+\kappa \sigma_{u u}\right) & \sigma_{Y Y}\left(\sigma_{x x}+\kappa \sigma_{u u}\right)+\sigma_{X Y}^{2} & 0 \\
0 & 0 & 2 \nu \kappa \sigma_{u u}^{2}
\end{array}\right] .
$$

De (3.61), podemos aplicar o teorema do limite central de Liapounov:

$$
\frac{\sum_{t=1}^{n} U_{3 t}}{\sqrt{\frac{\sum_{t=1}^{n} \operatorname{Var}\left(U_{3 t}\right)}{n^{2}}}}=\frac{\sqrt{n}\left(\frac{\sum_{t=1}^{n} U_{3 t}}{n}\right)}{\sqrt{\frac{\sum_{t=1}^{n}\left(\mathbf{a}^{\prime} \Gamma_{3 t} \mathbf{a}\right)}{n^{2}}}} \stackrel{\mathrm{D}}{\longrightarrow} N(0,1),
$$

onde

$$
\begin{aligned}
\sum_{t=1}^{n} \operatorname{Var}\left(U_{3} t\right)= & \sum_{t=1}^{n} \mathbf{a}^{\prime} \Gamma_{3 t} \mathbf{a}=2 a_{1}^{2}\left(\sum_{t=1}^{n} \sigma_{X X_{t}}^{2}\right)+4 a_{1} a_{2} \sigma_{\bar{X} Y}\left(\sum_{t=1}^{n} \sigma_{X X_{t}}\right) \\
& +a_{2}^{2} \sigma_{Y Y}\left(\sum_{t=1}^{n} \sigma_{X X_{t}}\right)+n a_{2}^{2} \sigma_{\bar{X} Y}^{2}+2 a_{3}^{2} \nu_{n}^{2} \sigma_{u u}^{2} \sum_{t=1}^{n}\left(r_{t}-1\right) .
\end{aligned}
$$

Temos também que (veja os cálculos no Apêndice-C.3) $E\left(U_{3 t}^{4}\right)<\infty$ (condição imposta por Ruppert (Gallo 1982)). 
Com a condição acima e assumindo a existência dos limites (3.45), (3.46) e (3.50), temos pelo lema de Gleser-Ruppert que:

$$
\sqrt{n}\left(\frac{\sum_{t=1}^{n} U_{3 t}}{n}\right) \stackrel{\mathrm{D}}{\longrightarrow} N\left(0, \mathbf{a}^{\prime} \Gamma_{3} \mathbf{a}\right) .
$$

De (3.55), (3.63) e aplicando o teorema de Cramér-Wold temos:

$$
\sqrt{n}\left[\left(m_{\bar{X} \bar{X}_{n}}, m_{\bar{X} Y_{n}}, \kappa_{n} S_{u u_{n}}\right)^{\prime}-\left(\sigma_{\bar{X} \bar{X}_{n}}, \sigma_{\bar{X} Y}, \kappa_{n} \sigma_{u u}\right)^{\prime}\right] \stackrel{\mathrm{D}}{\longrightarrow} N\left(0, \Gamma_{3}\right)
$$

Como

$$
\hat{\beta}_{N B 3}=\frac{m_{\bar{X} Y_{n}}}{m_{\bar{X} \bar{X}_{n}}-\left(\frac{\sum_{t=1}^{n} \frac{1}{r_{t}}}{n}\right) S_{u u 2 n}}=\frac{m_{\bar{X} Y_{n}}}{m_{\bar{X} \bar{X}_{n}}-\kappa_{n} S_{u u 2_{n}}},
$$

pelas definições dadas em (3.29), temos

$$
\dot{g}\left(\theta_{3}\right)=\sigma_{x x}^{-1}(-\beta, 1, \beta)^{\prime}, \quad \text { onde } \quad \theta_{3}=\left(\left(\sigma_{\bar{X} \bar{X}_{n}}, \sigma_{\bar{X} Y}, \kappa_{n} \sigma_{u u}\right)^{\prime},\right.
$$

logo, pelo método delta (Sen e Singer, 1993), temos

$$
\sqrt{n}\left(\hat{\beta}_{N B 3}-\beta\right) \stackrel{\mathrm{D}}{\longrightarrow} N\left(0, \gamma_{3}^{2}\right), \quad \text { onde } \gamma_{3}^{2}=(\dot{g}(\theta))^{\prime} \boldsymbol{\Gamma}_{3} g(\theta) \text {. }
$$

Após algumas manipulações algébricas obtemos (veja Apêndice C.5)

$$
\gamma_{3}^{2}=\sigma_{x x}^{-2}\left\{2 \beta^{2} \sigma_{\bar{X} \bar{X}}^{* 2}-4 \beta \sigma_{\bar{X} Y} \sigma_{\bar{X} \bar{X}}+\sigma_{\bar{X} \bar{X}} \sigma_{Y Y}+\sigma_{\bar{X} Y}^{2}+2 \nu \kappa \beta^{2} \sigma_{u u}^{2}\right\}
$$

com

$$
\begin{aligned}
\sigma_{\bar{X} \bar{X}}^{* 2} & =\sigma_{x x}^{2}+2 \kappa \sigma_{x x} \sigma_{u u}+\nu_{6} \sigma_{u u}^{2}, \quad \sigma_{\bar{X} \bar{X}}=\sigma_{x x}+\kappa \sigma_{u u}, \\
\sigma_{\bar{X} Y} & =\beta \sigma_{x x} \text { e } \sigma_{Y Y}=\beta^{2} \sigma_{x x}+\sigma_{e e} .
\end{aligned}
$$

Portanto, temos

$$
\gamma_{3}^{2}=\sigma_{x x}^{-2}\left[\kappa \beta^{2} \sigma_{x x} \sigma_{u u}+\left(\sigma_{x x}+\kappa \sigma_{u u}\right) \sigma_{e e}+2 \beta^{2} \sigma_{u u}^{2}\left(\nu_{6}+\nu \kappa\right)\right] .
$$




\subsection{Estimadores de $\beta$ corrigidos e ponderados}

Nesta seção examinamos os estimadores alternativos da declividade $\beta$, com as modificações propostas por Fuller (1987, Seções 2.5 e 3.1.2) e recomendadas por Schafer e Purdy (1996). Estes estimadores serão comparados (por simulações; os resultados serão apresentados na última seção) com os EMVs obtidos via algoritmo EM, desenvolvido em Schafer e Purdy (1996), para o número de replicações da variável explanatória $r_{t}$ variável. Quando $r_{t}$ e $s_{t}$ (número de replicações da variável resposta) forem constantes, estes estimadores serão comparados com os EMVs obtidos pelo método de estimação desenvolvido em Chan e Mak (1979).

Segundo Fuller (1987, Seção 2.5), o EMV de $\beta$, sob a suposição (S-1), isto é, com $\sigma_{u u}$ conhecido e $\sigma_{x x}>0$, é dado por

$$
\hat{\beta}=\frac{m_{X Y}}{m_{X X}-\sigma_{u u}} .
$$

Este estimador é consistente e tem distribuição limite normal, sob condições de regularidade bastante amplas (estas condições podem ser encontradas, por exemplo, em Sen e Singer (1993)), mas não necessariamente, tem expectância e primeiros momentos finitos para pequenas amostras. As modificações propostas por Fuller têm em vista corrigir estes "defeitos" e outros, como os vícios de primeira ordem apontados em Schafer e Purdy (1996), e melhorar as propriedades para pequenas amostras relativamente ao EMV (3.64). Apresentamos, a seguir, os argumentos apresentados em Fuller (1987, seção 2.5), que justificam e fundamentam tais modificações.

O EMV dado por (3.64), segundo Fuller, é uma razão entre duas variáveis aleatórias, $m_{X Y}$, e $m_{X X}-\sigma_{u u}$; mas tal razão é tipicamente um estimador viciado de $\beta$. Com efeito, a expectância do estimador em (3.64) não está definida (veja Fuller, 1987; Ex. 1.2.13 ). A sua proposta é, então, mostrar que é possível modificar este estimador para produzir um que seja "quase" não-viciado. Os procedimentos empregados para consecução de tal fim são justificados e apresentados na seção 2.5 do livro citado. Reapresentamos aqui, os principais aspectos destas modificações. Definindo, conforme Fuller, o seguinte estimador alternativo (Fuller, 1987, equação 2.5.3)

$$
\tilde{\beta}=\left[\tilde{H}_{x x}+\delta\left(n^{-1}\right) \sigma_{u u}\right]^{-1} m_{X Y},
$$


em que $\delta>0$ é um número real fixo a ser determinado,

$$
\tilde{H}_{x x}=\left\{\begin{array}{lll}
m_{X X}-\sigma_{u u}, & \text { se } & \hat{\lambda} \geq 1+(n-1)^{-1} \\
m_{X X}-\left[\hat{\lambda}-(n-1)^{-1}\right] \sigma_{u u}, & \text { se } & \hat{\lambda}<1+(n-1)^{-1}
\end{array}\right.
$$

e $\hat{\lambda}$ é raiz da equação determinantal

$$
\left\|m_{Z Z}-\lambda \cdot \operatorname{diag}\left\{0, \sigma_{u u}\right\}\right\|=0 .
$$

As implicações destas modificações levam a um estimador no qual a existência dos primeiros momentos, como veremos a seguir, está garantida.

Com efeito, observemos, inicialmente que o estimador de $\sigma_{x x}$, denotado por $\widehat{H}_{x x}$, nunca é menor que $(n-1)^{-1} \sigma_{u u}$, ou seja, $\widehat{H}_{x x} \geq(n-1)^{-1} \sigma_{u u}$. De fato, se $\hat{\lambda} \geq 1+(n-1)^{-1} \Longrightarrow H_{x x}=m_{X X}-\sigma_{u u}$.

$\mathrm{Da}$ equação (3.67) temos que

$$
\begin{aligned}
& \hat{\lambda}=\sigma_{u u}^{-1}\left[m_{X X}-\frac{m_{Y X}^{2}}{m_{Y Y}}\right] \geq 1+(n-1)^{-1} \Longrightarrow m_{X X}-\frac{m_{Y X}^{2}}{m_{Y Y}} \geq \\
& \sigma_{u u}\left[1+(n-1)^{-1}\right] \Longrightarrow m_{X X}-\sigma_{u u}=\widehat{H}_{x x} \geq \frac{m_{Y X}^{2}}{m_{Y Y}}+(n-1)^{-1} \sigma_{u u} .
\end{aligned}
$$

Por outro lado temos, se

$$
\begin{aligned}
& \hat{\lambda}<1+(n-1)^{-1} \Longrightarrow \widehat{H}_{x x}=m_{X X}-\left[\hat{\lambda}-(n-1)^{-1}\right] \sigma_{u u}=m_{X X}- \\
& \left\{\sigma_{u u}^{-1}\left(m_{X X}-m_{Y Y}^{-1} m_{Y X}^{2}\right)-(n-1)^{-1}\right\} \sigma_{u u}=m_{Y Y}^{-1} m_{Y X}^{2}+(n-1)^{-1} \sigma_{u u}= \\
& =\frac{m_{Y X}^{2}}{m_{Y Y}}+(n-1)^{-1} \sigma_{u u} \geq(n-1)^{-1} \sigma_{u u} .
\end{aligned}
$$

Portanto, a modificação adicional associada a $\delta$ produz um denominador, no estimador de $\beta$, que nunca é menor que $(n-1)^{-1}(1+\delta) \sigma_{u u}$, pois, $\left[\widehat{H}_{x x}+\delta(n-1)^{-1} \sigma_{u u}\right] \leq(n-1)^{-1} \sigma_{u u}+\delta(n-1)^{-1} \sigma_{u u}=(n-1)^{-1}(1+\delta) \sigma_{u u}$.

Como o denominador é limitado inferiormente por um número positivo o estimador tem primeiro momento. $\mathrm{O}$ valor de $\delta$ pode ser determinado minimizando-se o erro quadrático médio, ou equivalentemente, a expressão do vício dado em Fuller (1987, Teorema 2.5):

$$
E(\tilde{\beta}-\beta)=(n-1)^{-1} \sigma_{x x}^{-1}\left\{\left(\delta-2-2 \sigma_{x x}^{-1} \sigma_{u u}\right)\right\} \sigma_{u v}+O\left(n^{-2}\right) .
$$


Isto motiva tomarmos um $\delta=2+2 m_{X X}^{-1} \sigma_{u u}$. A quantidade $m_{X X}^{-1} \sigma_{u u}$ é um estimador viciado da razão $\sigma_{x x}^{-1} \sigma_{u u}$, mas é preferido a outros estimadores porque tem uma variância menor. Outros valores sugeridos por Fuller (1987) são: $\delta=2$ e $\delta=5$.

A equação (3.67) está associada à condição que garante valores positivos para estimadores de $\sigma_{e e}$ e $\sigma_{x x}$. Segundo Fuller (1987), se resolvermos a equação determinantal $\left\|\Sigma_{\mathbf{Z Z}}-\lambda_{i} \mathbf{D}_{i i}\right\|=0$ para $\lambda_{i}$, na qual $D_{i i}$ é uma matriz diagonal com o i-ésimo elemento igual a 1 e todas as outras entradas iguais a zero, obtemos a cota superior para a variância do erro do i-ésimo componenente de $\mathbf{Z}_{t}=\left(Y_{t}, 1, X_{t}\right)$. O coeficiente $\lambda_{i}$ é a média quadrática residual da regressão populacional do i-ésimo elemento de $Z_{t}$ sobre os remanescentes elementos de $\mathbf{Z}_{t}$. Assim uma estimativa da cota superior de $\sigma_{u u}$ seria:

$$
\left[\begin{array}{cc}
m_{Y Y} & m_{X Y} \\
m_{Y X} & m_{X X}
\end{array}\right]-\widehat{\lambda}_{2}\left[\begin{array}{ll}
0 & 0 \\
0 & 1
\end{array}\right]=0 \Longrightarrow\|\| \begin{array}{cc}
m_{Y Y} & m_{X Y} \\
m_{Y X} & m_{X X}-\widehat{\lambda_{2}}
\end{array} \|=0 .
$$

De (3.68) resulta que $\widehat{\lambda}_{2}=m_{X X}-\frac{m_{X Y}^{2}}{m_{Y Y}}$, que é uma estimativa de $\lambda_{2}=\sigma_{X X}-\frac{\sigma_{X Y}^{2}}{\sigma_{Y Y}}$. Mas $\lambda_{2}$ está associada ao erro de mensuração do segundo elemento de $\mathbf{Z}_{t}$, isto é $X_{t}$, logo $\widehat{\lambda}_{2}$ é uma estimativa da cota superior da $\operatorname{Var}\left(u_{t}\right)$, denotada por $\sigma_{u u}$. De acordo com Fuller (1987, Equação 1.2.4) a condição necessária e suficiente para que os estimadores de $\sigma_{x x}$ e $\sigma_{e e}$ sejam positivos é que

$$
\begin{aligned}
m_{Y Y}\left(m_{X X}-\sigma_{u u}\right)-m_{X Y}^{2}>0 & \Longrightarrow m_{X X}-\sigma_{u u}>\frac{m_{X Y}^{2}}{m_{Y Y}} \\
& \Longrightarrow m_{X X}-\frac{m_{X Y}^{2}}{m_{Y Y}}=\widehat{\lambda_{2}}>\sigma_{u u} .
\end{aligned}
$$

Estudos comparativos, por meio de histogramas amostrais, do estimador corrigido $\tilde{\beta}_{1}$ dado em (3.65) com o EMV dado em (3.64), para alguns valores de $\delta$, encontramse em Fuller (1987, Seção 2.5.1). Eles mostram a superioridade do estimador corrigido quando se considera tamanho da amostra pequeno $(n=21)$, em 2000 amostras.

\subsubsection{Estimador $\hat{\beta}_{N B G}$ corrigido}

Quando as variâncias dos erros de mensuração $\left.\mathrm{a}_{t}=\left(e_{t}, 0, u_{t}\right)\right)^{\prime}$ são conhecidas, porém variam com t, o modelo introduzido por Fuller (1987, Cap.3), pode ser, assim, colocado 
(considerando as suas notações):

$$
\left(\begin{array}{lll}
x_{t} & e_{t}, u_{t}
\end{array}\right) \sim N\left[\left(\begin{array}{c}
\mu_{x} \\
0 \\
0
\end{array}\right), \operatorname{diag}\left\{\sigma_{x x}, \sigma_{\text {eett }}, \sigma_{\text {uutt }}\right\}\right] .
$$

O estimador do vetor paramétrico $\beta=(\alpha, \beta)^{\prime}$ proposto por Fuller (1987, eq. 3.1.19) é:

$$
\tilde{\beta}=\widehat{\mathrm{M}}_{x x}^{-1} \widehat{\mathrm{M}}_{x y}
$$

onde as matrizes

$$
\left(\widehat{\mathrm{M}}_{x y}, \widehat{\mathrm{M}}_{x x}\right)=n^{-1} \sum_{t=1}^{n}\left(\mathrm{X}_{t}^{\prime} Y_{t}, \mathrm{X}_{t}^{\prime} \mathrm{X}_{t}-\Sigma_{u u t t}\right)
$$

com

$$
\mathrm{X}_{t}=\left(1, X_{t}\right)^{\prime}=\left(1, x_{t}+u_{t}\right)^{\prime}=\left(1, x_{t}\right)^{\prime}+\left(0, u_{t}\right)^{\prime}=\mathrm{x}_{t}+\mathbf{u}_{t} \text {, e } \Sigma_{u u t t} \text { é a matriz }
$$

de covariância de $u_{t}$, ou seja,

$$
\Sigma_{u u t t}=\left[\begin{array}{cc}
0 & 0 \\
0 & \sigma_{u u t t}
\end{array}\right]
$$

Desenvolvendo-se a equação matricial (3.70) obtemos

$$
\tilde{\beta}=\frac{\sum_{t=1}^{n}\left(X_{t}-\bar{X}\right)\left(Y_{t}-\bar{Y}\right)}{\sum_{t=1}^{n}\left[\left(X_{t}-\bar{X}\right)^{2}-\sigma_{u u t t}\right]}, \quad \tilde{\alpha}=\bar{Y}-\tilde{\beta} \bar{X} .
$$

Quando as variâncias dos erros de mensuração $\Sigma_{a a}$ são desconhecidas, Fuller (1987, Seção 3.1.2) considera replicações das duas variáveis - resposta e explanatória - que geram um estimador $S_{a a}$ da matriz de covariâncias $\Sigma_{a a}$ dos erros nas variáveis, $\mathbf{a}_{t}=\left(e_{t}, 0, u_{t}\right)$. Este estimador, segundo Fuller, é obtido de uma fonte independente do vetor $\left(X_{t}, Y_{t}\right)$, e é distribuído como um múltiplo da matriz de Wishart com $d_{f}$ graus de liberdade (Rao 1973); sendo que $d_{f}$ é o número de elementos do subconjunto replicado da amostra, considerando duas determinações independentes e identicamente distribuídas. Portanto, com as suposições adotadas até aqui, e considerando o estimador $\mathrm{S}_{a a}$ obtido somente com replicações não-balanceadas da variável explanatória, podemos 
modificar o estimador $\tilde{\beta}(3.73)$, ao substituirmos $\sigma_{u u t t}$ por $S_{u u t t},\left(S_{u u t t}=S u u / r_{t}\right)$, e $X_{t}$ por $\bar{X}_{t}$, obtendo-se um estimador equivalente ao dado em Fuller (1987, eq. 3.1.33), que apresentamos a seguir, com o propósito de apreender e fixar notações, que serão necessárias mais adiante,

$$
\tilde{\beta}_{p}=\widetilde{\Sigma}_{x x}^{-1} n^{-1} \sum_{t=1}^{n} \overline{\mathrm{X}}_{t .}^{\prime} Y_{t}
$$

em que

$$
\begin{gathered}
\tilde{\mathbf{\Sigma}}_{x x}^{-1}=n^{-1}\left[\sum_{t=1}^{n} \overline{\mathbf{X}}_{t .}^{\prime} \overline{\mathbf{X}}_{t .}-\mathrm{S}_{u u} \sum_{t=1}^{n} \frac{1}{r_{t}}\right], \\
\overline{\mathbf{X}}_{t .}=\left(1, \bar{X}_{t .}\right), \quad \overline{\mathbf{u}}_{t .}=\left(1, \bar{u}_{t .}\right), \quad \bar{X}_{t .}=\frac{\sum_{j=1}^{r_{t}} X_{t j}}{r_{t}}, \quad \bar{X}_{. .}^{*}=\frac{\sum_{t=1}^{n} \bar{X}_{t .}}{n}, \\
\overline{\mathbf{X}}_{t .}^{\prime} \overline{\mathbf{X}}_{t .}=\left[\begin{array}{cc}
1 & \bar{X}_{t .} \\
\bar{X}_{t .} & \bar{X}_{t .}^{2}
\end{array}\right], \quad \overline{\mathbf{X}}_{t .}^{\prime} Y_{t}=\left[\begin{array}{c}
Y_{t} \\
\bar{X}_{t .} Y_{t}
\end{array}\right], \quad \mathrm{S}_{u u}=\left[\begin{array}{cc}
0 & 0 \\
0 & S_{u u}
\end{array}\right]
\end{gathered}
$$

$\mathrm{e}$

$$
S_{u u}=\frac{\sum_{t=1}^{n} \sum_{j=1}^{r_{t}}\left(X_{t j}-\bar{X}_{t .}\right)^{2}}{\sum_{t=1}^{n}\left(r_{t}-1\right)}
$$

Agora, desenvolvendo a equação matricial (3.74), obtemos

$$
\tilde{\boldsymbol{\beta}}_{p}=\left\{n^{-1}\left[\begin{array}{cc}
n & \sum_{t=1}^{n} \bar{X}_{t .} \\
\sum_{t=1}^{n} \bar{X}_{t .} & \sum_{t=1}^{n}\left(\bar{X}_{t .}^{2}-\frac{S u u}{r_{t}}\right)
\end{array}\right]\right\}^{-1} n^{-1}\left[\begin{array}{c}
\sum_{t=1}^{n} Y_{t} \\
\sum_{t=1}^{n} \bar{X}_{t .} Y_{t}
\end{array}\right]
$$

ou seja,

$$
\tilde{\beta}_{p}=\frac{\sum_{t=1}^{n}\left(\bar{X}_{t .}-\bar{X}_{. .}^{*}\right)\left(Y_{t}-\bar{Y} .\right)}{\sum_{t=1}^{n}\left[\left(\bar{X}_{t .}-\bar{X}_{. .}^{*}\right)^{2}-\frac{S u u}{r_{t}}\right]}=\frac{m_{\bar{X} Y}}{m_{\bar{X} \bar{X}}-\left[\frac{\sum_{t=1}^{n} \frac{1}{r_{t}}}{n}\right] S_{u u}}
$$

$\mathrm{e}$

$$
\tilde{\alpha}_{p}=\bar{X}_{. .}^{*}-\tilde{\beta}_{p} \bar{Y}
$$

Mas este estimador é exatamente o estimador $\hat{\beta}_{N B 3}$ dado em (3.49). Portanto, daqui em diante passaremos a tratá-lo com esta notação $\left(\hat{\beta}_{N B 3}\right)$. Ele desempenhará o papel de estimador preliminar para outros estimadores melhorados.

Na prática, o estimador $\hat{\beta}_{N B 3}$ dado em (3.76) pode passar por um ajustamento (para corrigir os vícios de primeiro grau e garantir a existência dos primeiros momentos) da maneira análoga à descrita no início desta seção. Este estimador ajustado é uma 
adptação do estimador da equação (3.1.2) em Fuller (1987). Schafer e Purdy (1996) referem-se a ele como um estimador corrigido de vícios de primeiro grau, decorrentes do uso de $\hat{A}^{-1} \hat{B}$ como um estimador de $[E(A)]^{-1} E(B)$, denotando-o por $\hat{\beta}_{b}$. Da equação (3.1.20) de Fuller(1987), que supõe $\boldsymbol{\Sigma}_{\text {aatt }}$ conhecida, porém dependente da t-ésima observação, temos:

$$
\begin{gathered}
\tilde{\boldsymbol{\beta}}_{b p}=\left(\begin{array}{c}
\tilde{\alpha}_{b p} \\
\tilde{\beta}_{b p}
\end{array}\right)=\tilde{\mathbf{H}}_{x x}^{-1} \tilde{\mathbf{H}}_{x y}, \\
\tilde{\mathbf{H}}_{x x}=\left\{\begin{array}{lll}
\sum_{t=1}^{n}\left[\mathbf{Z}_{t}^{\prime} \mathbf{Z}_{t}-\left(1-\delta n^{-1}\right) \Sigma_{a a t t t}\right], & \text { se } & \hat{\lambda}>1+n^{-1} \\
\sum_{t=1}^{n}\left[\mathbf{Z}_{t}^{\prime} \mathbf{Z}_{t}-\left(\hat{\lambda}-n^{-1}-\delta n^{-1}\right) \Sigma_{a a t t}\right], & \text { se } & \hat{\lambda} \leq 1+n^{-1},
\end{array}\right.
\end{gathered}
$$

em que $\hat{\lambda}$ é a menor raiz de

$$
\left|\sum_{t=1}^{n}\left(\mathrm{z}_{t}^{\prime} \mathbf{z}_{t}-\lambda \Sigma_{\text {aatt }}\right)\right|=0
$$

$\left.\mathbf{Z}_{t}=\left(Y_{t}, \mathbf{X}_{t}\right)=\left(Y_{t}, 1, X_{t}\right)\right)$, e $\delta$ é um número real a ser determinado, como anteriormente. Considere, ainda, as seguintes notações, utilizadas por Fuller:

$$
\mathbf{a}_{t}=\left(e_{t}, 0, u_{t}\right)=\left(e_{t}, \mathrm{u}_{t}\right),
$$

e

$$
\Sigma_{\text {aatt }}=\left[\begin{array}{ccc}
\sigma_{\text {eett }} & 0 & \sigma_{\text {eutt }} \\
0 & 0 & 0 \\
\sigma_{\text {uett }} & 0 & \sigma_{\text {uutt }}
\end{array}\right]=\left[\begin{array}{cc}
\sigma_{\text {eett }} & \Sigma_{\text {eutt }} \\
\Sigma_{\text {uett }} & \Sigma_{\text {uutt }}
\end{array}\right] .
$$

Considere, agora, os seguintes cálculos preliminares, como forma de fixar notações:

$$
\mathbf{Z}_{t}^{\prime} \mathbf{Z}_{t}=\left[\begin{array}{c}
Y_{t} \\
1 \\
X_{t}
\end{array}\right]\left[\begin{array}{lll}
Y_{t} & 1 & X_{t}
\end{array}\right]=\left[\begin{array}{ccc}
Y_{t}^{2} & Y_{t} & Y_{t} X_{t} \\
Y_{t} & 1 & X_{t} \\
X_{t} Y_{t} & X_{t} & X_{t}^{2}
\end{array}\right]=\left[\begin{array}{cc}
Y_{t}^{2} & Y_{t} \mathbf{X}_{t}^{\prime} \\
Y_{t} \mathbf{X}_{t} & \mathbf{X}_{t}^{\prime} \mathbf{X}_{t}
\end{array}\right]
$$

Portanto, se $\lambda>1+n^{-1}$, segue-se que

$$
\tilde{\mathbf{H}}_{z z}=\left[\begin{array}{ccc}
\sum_{t=1}^{n}\left[Y_{t}^{2}-\left(1-\delta n^{-1}\right) \sigma_{e e t t}\right] & \sum_{t=1}^{n} Y_{t} & \sum_{t=1}^{n} X_{t} Y_{t} \\
\sum_{t=1}^{n} Y_{t} & n & \sum_{t=1}^{n} X_{t} \\
\sum_{t=1}^{n} X_{t} Y_{t} & \sum_{t=1}^{n} X_{t} & \sum_{t=1}^{n}\left[X_{t}^{2}-\left(1-\delta n^{-1}\right) \sigma_{u u t t}\right]
\end{array}\right]
$$




$$
=\left[\begin{array}{cc}
\sum_{t=1}^{n}\left[Y_{t}^{2}-\left(1-\delta n^{-1}\right) \sigma_{e e t t}\right] & \tilde{\mathbf{H}}_{y x} \\
\tilde{\mathbf{H}}_{x y} & \tilde{\mathbf{H}}_{x x}
\end{array}\right] .
$$

No caso da matriz de covariância dos erros de mensuração desconhecida, com replicações nas duas variáveis, $\left(Y_{t}, X_{t}\right)$, o estimador $\mathbf{S}_{a a}$ de $\boldsymbol{\Sigma}_{a a}$ proposto por Fuller (1987, eq. 3.1.32) é

$$
\mathbf{S}_{a a}=\frac{\sum_{t=1}^{n} \sum_{j=1}^{r_{t}}\left(\mathbf{Z}_{t j}-\overline{\mathbf{Z}}_{t}\right)^{\prime}\left(\mathbf{Z}_{t j}-\overline{\mathbf{Z}}_{t}\right)}{\sum_{t=1}^{n}\left(r_{t}-1\right)},
$$

ou na forma matricial,

$$
\mathrm{S}_{a a}=\left[\begin{array}{ccc}
S_{e e} & 0 & S_{e u} \\
0 & 0 & 0 \\
S_{u e} & 0 & S_{u u}
\end{array}\right]=\left[\begin{array}{cc}
S_{e e} & \mathrm{~S}_{e u} \\
\mathrm{~S}_{u e} & \mathrm{~S}_{u u}
\end{array}\right]
$$

em que

$$
\begin{gathered}
S_{e e}=\frac{\sum_{t=1}^{n} \sum_{j=1}^{r_{t}}\left(Y_{t j}-\bar{Y}_{t .}\right)^{2}}{\sum_{t=1}^{n}\left(r_{t}-1\right)} \\
S_{u e}=\frac{\sum_{t=1}^{n} \sum_{j=1}^{r_{t}}\left(Y_{t j}-\bar{Y}_{t .}\right)\left(X_{t j}-\bar{X}_{t .}\right)}{\sum_{t=1}^{n}\left(r_{t}-1\right)}=S_{e u}
\end{gathered}
$$

e $S_{u u}$ já foi dado anteriormente. Consideremos, ainda, as notações:

em que

$$
\mathrm{S}_{\text {aatt }}=\left[\begin{array}{ccc}
S_{\text {eett }} & 0 & S_{\text {eutt }} \\
0 & 0 & 0 \\
S_{\text {uett }} & 0 & S_{\text {uutt }}
\end{array}\right]=\left[\begin{array}{ll}
S_{\text {eett }} & \mathrm{S}_{\text {eutt }} \\
\mathrm{S}_{\text {uett }} & \mathrm{S}_{\text {uutt }}
\end{array}\right]
$$

$$
S_{\text {eet }}=\frac{S e e}{r_{t}}, \quad S_{e u t t}=S_{u e t t}=\frac{S_{u e}}{r_{t}}=\frac{S_{e u}}{r_{t}} \quad \text { e } \quad S_{u u t t}=\frac{S_{u u}}{r_{t}} .
$$

Sob o modelo adotado, temos que levar em conta somente os elementos $\sigma_{\text {uutt }}$ e $\sigma_{\text {eett }}$ da matriz $\Sigma_{\text {aatt }}$, sendo $\sigma_{\text {eutt }}=0$. Por outro lado, considerando as replicações só da variável explanatória, o único estimador a considerar é $S_{u u t t}$, sendo as outras entradas da matriz $S_{a a t t}$ iguais a zero. Ou seja, 


$$
\mathrm{S}_{a a t t}=\left[\begin{array}{ccc}
0 & 0 & 0 \\
0 & 0 & 0 \\
0 & 0 & \frac{S_{u u}}{r_{t}}
\end{array}\right]=\left[\begin{array}{cc}
S_{\text {eett }} & \mathrm{S}_{\text {eutt }} \\
\mathrm{S}_{\text {uett }} & \mathrm{S}_{\text {uutt }}
\end{array}\right] .
$$

Para assegurar que o erro cometido ao estimar $\Sigma_{a a}$ entre na matriz de covariância da distribuição limite, Fuller (1987) supõe que este erro seja da mesma ordem que o de $M_{\mathrm{zz}}$. Isto é satisfeito se os graus de liberdade $\left(d_{f}\right)$ de $\mathrm{S}_{a a}$ for proporcional a $\mathrm{n}$, isto é, $\lim _{n \rightarrow \infty} \frac{n}{d_{f}}=\mu$, um número fixo. Incorporando as informações das replicações de $x_{t}$, consideremos o seguinte vetor de variáveis observáveis;

$$
\mathbf{Z}_{t}^{*}=\left(Y_{t}, \overline{\mathbf{X}}_{t}\right)^{\prime}=\left(Y_{t}, 1, \bar{X}_{t}\right)^{\prime} \quad \text { e } \quad \mathbf{a}_{t}^{*}=\left(e_{t}, \overline{\mathbf{u}}_{t}\right)^{\prime}=\left(e_{t}, 1, \bar{u}_{t}\right)^{\prime}
$$

que aplicados na matriz $\tilde{\mathbf{H}}_{z z}$, vem

$$
\begin{gathered}
\widehat{\mathbf{H}}_{z z}^{*}=\left[\begin{array}{ccc}
\sum_{t=1}^{n} Y_{t}^{2} & \sum_{t=1}^{n} Y_{t} & \sum_{t=1}^{n} \bar{X}_{t} Y_{t} \\
\sum_{t=1}^{n} Y_{t} & n & \sum_{t=1}^{n} \bar{X}_{t} \\
\sum_{t=1}^{n} \bar{X}_{t} Y_{t} & \sum_{t=1}^{n} \bar{X}_{t} & \sum_{t=1}^{n}\left[\bar{X}_{t}^{2}-\left(1-\delta n^{-1}\right) S_{u u t t}\right]
\end{array}\right]=\left[\begin{array}{cc}
\sum_{t=1}^{n} Y_{t}^{2} & \widehat{\mathbf{H}}_{y x}^{*} \\
\widehat{\mathbf{H}}_{x y}^{*} & \widehat{\mathbf{H}}_{x x}^{*}
\end{array}\right] \\
=\left[\begin{array}{cc}
\sum_{t=1}^{n} Y_{t}^{2} & \widehat{\mathbf{H}}_{y x}^{*} \\
\widehat{\mathbf{H}}_{x y}^{*} & \widehat{\mathbf{H}}_{x x}^{*}
\end{array}\right] .
\end{gathered}
$$

Portanto, para $\lambda>1+n^{-1}$, segue-se que

$$
\widehat{\beta}_{b}=\left(\begin{array}{c}
\widehat{\alpha}_{b} \\
\widehat{\beta}_{b}
\end{array}\right)=\left(\widehat{\mathbf{H}}_{x x}^{*}\right)^{-1} \widehat{\mathbf{H}}_{x y}^{*},
$$

ou seja,

$$
\begin{aligned}
\widehat{\beta}_{b}= & \frac{\sum_{t=1}^{n}\left(\bar{X}_{t .}-\bar{X}_{. .}^{*}\right)\left(Y_{t}-\bar{Y} .\right)}{\sum_{t=1}^{n}\left[\left(\bar{X}_{t .}-\bar{X}_{. .}^{*}\right)^{2}-\left(1-\delta n^{-1}\right) \frac{S u u}{r_{t}}\right]}=\frac{m_{\bar{X} Y}}{m_{\bar{X} \bar{X}}-\left(1-\delta n^{-1}\right)\left[\frac{\left.\sum_{t=1}^{n} \frac{1}{r_{t}}\right] S_{u u}}{n}\right.}, \\
\mathrm{e} & \widehat{\alpha}_{b}=\bar{X}_{. .}^{*}-\widehat{\beta}_{b} \bar{Y} .
\end{aligned}
$$

Para $\hat{\lambda} \leq 1+n^{-1}$, substituir $\left(1-\delta n^{-1}\right)$ por $\left(\hat{\lambda}-n^{-1}-\delta n^{-1}\right)$ na equação (3.80). em que $\hat{\lambda}$ é a menor raíz de: 


$$
\left\|\sum_{t=1}^{n}\left(\mathbf{Z}_{t}^{*^{\prime}} \mathbf{Z}_{t}^{*}-\lambda \mathrm{S}_{\text {aatt }}\right)\right\|=0, \text { onde }\|A\| \text { denota determinante de } \mathrm{A} .
$$

O estimador $\widehat{\beta}_{b}$ de (3.92) é o $\widehat{\beta}_{N B 3}$ corrigido. Resolvendo a equação determinantal acima obtemos

$$
\begin{gathered}
\left\|\begin{array}{ccc}
\sum_{t=1}^{n} Y_{t}^{2} & \sum_{t=1}^{n} Y_{t} & \sum_{t=1}^{n} \bar{X}_{t} Y_{t} \\
\sum_{t=1}^{n} Y_{t} & n & \sum_{t=1}^{n} \bar{X}_{t} \\
\sum_{t=1}^{n} \bar{X}_{t} Y_{t} & \sum_{t=1}^{n} \bar{X}_{t} & \sum_{t=1}^{n}\left(\bar{X}_{t}^{2}-\lambda S_{u u t t}\right)
\end{array}\right\|=0 \Longrightarrow \\
{\left[\sum_{t=1}^{n} \bar{X}_{t}^{2}-\lambda\left(\frac{\sum_{t=1}^{n} \frac{1}{r_{t}}}{n}\right) S_{u u}\right] n \sum_{t=1}^{n} Y_{t}^{2}+2\left(\sum_{t=1}^{n} \bar{X}_{t} Y_{t}\right)\left(\sum_{t=1}^{n} \bar{X}_{t}\right)\left(\sum_{t=1}^{n} Y_{t}\right)} \\
-n\left(\sum_{t=1}^{n} \bar{X}_{t} Y_{t}\right)^{2}-\left(\sum_{t=1}^{n} \bar{X}_{t .}\right)^{2}\left(\sum_{t=1}^{n} Y_{t}^{2}\right) \\
-\left(\sum_{t=1}^{n} Y_{t}\right)^{2}\left[\sum_{t=1}^{n} \bar{X}_{t}^{2}-\lambda\left(\frac{\sum_{t=1}^{n} \frac{1}{r_{t}}}{n}\right) S_{u u}\right]=0 \Longrightarrow \\
-n^{2} \lambda m_{Y Y}\left(\frac{\sum_{t=1}^{n} \frac{1}{r_{t}}}{n}\right)^{n} S_{u u}+n^{2} m_{Y Y} \sum_{t=1}^{n} \bar{X}_{t .}^{2}-n^{2} m_{\bar{X} Y}\left(\sum_{t=1}^{n} \bar{X}_{t} Y_{t}\right)+ \\
+n^{2}\left(\sum_{t=1}^{n} \bar{X}_{t} Y_{t}\right)\left(\bar{X}_{. .}^{*}\right)\left(\bar{Y}_{.}\right)-n^{2}\left(\bar{X}_{. .}^{*}\right)^{2}\left(\sum_{t=1}^{n} Y_{t}^{2}\right)=0
\end{gathered}
$$

Após algumas simplificações algébricas da equação determinantal acima obtemos

$$
\widehat{\lambda}=\left[\left(\frac{\sum_{t=1}^{n} \frac{1}{r_{t}}}{n}\right) S_{u u}\right]^{-1}\left(m_{\bar{X} \bar{X}}-\frac{m_{Y \bar{X}}^{2}}{m_{Y Y}}\right)
$$

em que

$$
\begin{aligned}
& m_{\bar{X} \bar{X}}=\frac{\sum_{t=1}^{n}\left(\bar{X}_{t .}-\bar{X}_{. .}^{*}\right)^{2}}{n}, \quad m_{\bar{X} Y}=\frac{\sum_{t=1}^{n}\left(\bar{X}_{t .}-\bar{X}_{. .}^{*}\right)\left(Y_{t}-\bar{Y}\right)}{n} \\
& m_{Y Y}=\frac{\sum_{t=1}^{n}\left(Y_{t}-\bar{Y}\right)^{2}}{n}
\end{aligned}
$$


Os valores de $\delta$ são escolhidos de tal forma que minimize os vícios de primeira ordem. Conforme a discussão feita anteriormente podemos adotar os valores $\delta=2, \delta=5$ ou $\delta=2+2\left(\sum_{t=1}^{n} r_{t}^{-1} / n\right) S_{u u} / m_{\bar{X} \bar{X}}$.

\section{Observações finais:}

- O $\tilde{\beta}$ dado em (3.73) é o estimador de $\beta$ quando as variâncias dos erros de mensuração das variáveis são conhecidas, porém variam com t.

- Quando as variâncias dos erros de mensuração são desconhecidas e variam com t, temos o estimador $\tilde{\beta}_{p}$ dado em (3.76), que coincide com o estimador $\hat{\beta}_{N B 3}$.

- O estimador $\tilde{\beta}_{b p}$ dado em (3.77) é o $\tilde{\beta}$ "corrigido".

- O estimador $\tilde{\beta}_{b}$ dado em (3.80) é o $\hat{\beta}_{N B 3}$ "corrigido".

De acordo com o Fuller (1987, teor. 3.1.1) o estimador $\tilde{\beta}$ de (3.73) é normalmente distribuído no limite. A distribuição limite de $\sqrt{n}\left(\tilde{\beta}_{b p}-\beta\right)$ é a mesma de $\sqrt{n}(\tilde{\beta}-\beta)$. Este estimador $\tilde{\beta}_{b p}$ é construído considerando $\Sigma_{a a t t}$ conhecida, portanto não usa as replicações de variáveis, mas, por outro lado, sem a suposição de normalidade para os erros de mensuração. A distribuição limite de $\widehat{\beta}_{b}$ coincide com a do $\hat{\beta}_{N B 3}$. O estimador $\widehat{\beta}_{b}$ de (3.80) será utilizado para comparações com os estimadores de máxima verossimilhança obtidos no Capítulo 2 .

\subsubsection{Estimadores ponderados melhorados}

Um problema mais grave para o estimador $\widehat{\beta}_{b}$ em (3.92), apontado por Fuller (1987, Seção 3.1) e ressaltado em Schafer e Purdy (1996), é o uso de pesos iguais, independente do número de replicações das variáveis. Fuller (1987) propõe duas versões com pesos melhorados, que denotamos por $\widehat{\beta}_{b w}$ e $\widehat{\beta}_{w}$. O estimador $\widehat{\beta}_{w}$ é um dos estimadores utilizados em Schafer e Purdy (1996) para um estudo comparativo com o EMV de $\beta$ obtido pela replicação da variável explanatória, via algoritmo EM; o outro é $\widehat{\beta}_{b}$ em (3.92).

A nossa proposta aqui, é adaptar os estimadores ponderados melhorados desenvolvidos em Fuller (1987, Seção 3.1), utilizando, para isso, replicações da variável explanatória 
e as suposição adotadas anteriormente. Neste sentido, apresentamos, a seguir, os principais aspectos e argumentos, relativamente ao nosso interesse, desenvolvidos em Fuller (1987).

Na primeira versão os pesos são baseados no método de estimação por mínimos quadrados generalizados (EMQG), usando o estimador $\tilde{\beta}_{N B 3}$ (3.70), como um estimador preliminar. A motivação para tal peso é dada em Fuller (1987). Lembremos que podemos escrever

$$
Y_{t}=\alpha+\beta X_{t}+v_{t}, \quad v_{t}=e_{t}-\beta u_{t} .
$$

A variância de $v_{t}$, em (3.83), é análoga a variância do erro na equação para a t-ésima observação de um modelo linear. Conseqüentemente, é razoável construir um EMQG da variância de $v_{t}, \sigma_{v v t t}$, para ponderar as observações. Tal estimador ponderado (Fuller, 1987, eq. 3.1.26) é dado por

$$
\tilde{\beta}_{b w}=\left[\sum_{t=1}^{n} \tilde{\sigma}_{v v t t}^{-1}\left(\mathbf{X}_{t}^{\prime} \mathbf{X}_{t}-\Sigma_{u u t t}\right)\right]^{-1} \sum_{t=1}^{n} \tilde{\sigma}_{v v t t}^{-1}\left(\mathbf{X}_{t}^{\prime} Y_{t}-\Sigma_{u e t t}\right),
$$

em que $\tilde{\sigma}_{v v t t}=\sigma_{\text {eett }}-2 \tilde{\boldsymbol{\beta}}_{N B 3}^{\prime} \Sigma_{u e t t}+\tilde{\beta}_{N B 3}^{\prime} \Sigma_{u u t t} \tilde{\beta}_{N B 3}, \operatorname{com} \tilde{\beta}_{N B 3}=\left(\alpha_{N B 3}, \beta_{N B 3}\right)^{\prime}$. Fazendo as adaptações apropriadas, conforme a proposta acima citada, e substituindo os parâmetros por estimadores consistentes e as modificações para corrigir os vícios de primeira ordem e garantir a existência dos primeiros momentos, obtemos

$$
\widehat{\beta}_{b w}=\left[\sum_{t=1}^{n} \widehat{\sigma}_{v v t t}^{-1}\left(\mathbf{X}_{t}^{\prime} \mathbf{X}_{t}-\left(1-\delta n^{-1}\right) \mathrm{S}_{u u t t}\right)\right]^{-1} \sum_{t=1}^{n} \widehat{\sigma}_{v v t t}^{-1}\left(\mathbf{X}_{t}^{\prime} Y_{t}\right)
$$

em que

$$
\widehat{\sigma}_{v v t t}=\widehat{\sigma}_{e e t t}+\tilde{\beta}_{N B 3}^{2} \frac{S_{u u}}{r_{t}}, \quad \Sigma_{u e t t}=0, \quad \widehat{\Sigma_{u u t t}}=\mathrm{S}_{u u t t},
$$

se $\widehat{\lambda}>1+n^{-1}$. Quando $\hat{\lambda} \leq 1+n^{-1}$ substituir $\left(1-\delta n^{-1}\right)$ por $\left(\widehat{\lambda}-n^{-1}-\delta n^{-1}\right)$ na equação (3.85), na qual, $\widehat{\lambda}$ é a menor raíz da equação determinantal

$$
\left\|\sum_{t=1}^{n} \widehat{\sigma}_{v v t t}^{-1}\left(\mathbf{Z}_{t}^{\prime} \mathbf{Z}_{t}-\lambda \mathbf{S}_{a a t t}\right)\right\|=0 .
$$

Agora, vamos desenvolver a equação matricial (3.85). Considerando $\widehat{\lambda}>1+n^{-1}$, temos 


$$
\begin{gathered}
\sum_{t=1}^{n} \hat{\sigma}_{v v t t}^{-1}\left[\mathrm{X}_{t}^{\prime} \mathrm{X}_{t}-\left(1-\delta n^{-1}\right) \mathrm{S}_{u u t t}\right]= \\
\sum_{t=1}^{n} \widehat{\sigma}_{v v t t}^{-1}\left[\mathrm{X}_{t}^{\prime} \mathrm{X}_{t}-\left(1-\delta n^{-1}\right) \mathrm{S}_{u u t t}\right]=\sum_{t=1}^{n} \widehat{\sigma}_{v v t t}^{-1}\left\{\left[\begin{array}{cc}
1 & \bar{X}_{t .} \\
\bar{X}_{t .} & \bar{X}_{t .}^{2}
\end{array}\right]-\left(1-\delta n^{-1}\right)\left[\begin{array}{cc}
0 & 0 \\
0 & S_{u u t t}
\end{array}\right]\right\} \\
=\sum_{t=1}^{n} \hat{\sigma}_{v v t t}^{-1}\left\{\left[\begin{array}{cc}
1 & \bar{X}_{t .} \\
\bar{X}_{t .} & \bar{X}_{t .}^{2}-\left(1-\delta n^{-1}\right) S_{u u t t}
\end{array}\right]\right\} \\
=\left[\begin{array}{cc}
\sum_{t=1}^{n} \hat{\sigma}_{v v t t}^{-1} & \sum_{t=1}^{n} \widehat{\sigma}_{v v t t}^{-1} \bar{X}_{t .} \\
\sum_{t=1}^{n} \hat{\sigma}_{v v t t}^{-1} \bar{X}_{t .} & \sum_{t=1}^{n} \widehat{\sigma}_{v v t t}^{-1}\left[\bar{X}_{t .}^{2}-\left(1-\delta n^{-1}\right) S_{u u t t}\right]
\end{array}\right] .
\end{gathered}
$$

Por outro lado temos

$$
\sum_{t=1}^{n} \widehat{\sigma}_{\text {vvtt }}^{-1}\left(\mathrm{X}_{t}^{\prime} Y_{t}\right)=\sum_{t=1}^{n} \widehat{\sigma}_{\text {vvtt }}^{-1}\left[\begin{array}{c}
1 \\
\bar{X}_{t .}
\end{array}\right] Y_{t}=\left[\begin{array}{c}
\sum_{t=1}^{n} \widehat{\sigma}_{\text {vvtt }}^{-1} Y_{t} \\
\sum_{t=1}^{n} \widehat{\sigma}_{v v t t}^{-1} \bar{X}_{t .} Y_{t}
\end{array}\right]
$$

A inversa da matriz em (3.86) é:

$$
\begin{gathered}
\left\{\left(\sum_{t=1}^{n} \widehat{\sigma}_{v v t t}^{-1}\right)\left(\sum_{t=1}^{n} \hat{\sigma}_{v v t t}^{-1}\left[\bar{X}_{t .}^{2}-\left(1-\delta n^{-1}\right) S_{u u t t}\right]\right)-\left(\sum_{t=1}^{n} \widehat{\sigma}_{v v t t}^{-1} \bar{X}_{t .}\right)^{2}\right\}^{-1} \times \\
\times\left[\begin{array}{cc}
\sum_{t=1}^{n} \hat{\sigma}_{v v t t}^{-1}\left[\bar{X}_{t .}^{2}-\left(1-\delta n^{-1}\right) S_{u u t t}\right] & -\sum_{t=1}^{n} \widehat{\sigma}_{v v t t}^{-1} \bar{X}_{t .} \\
-\sum_{t=1}^{n} \widehat{\sigma}_{v v t t}^{-1} \bar{X}_{t .} & \sum_{t=1}^{n} \widehat{\sigma}_{v v t t}^{-1}
\end{array}\right]
\end{gathered}
$$

Agora, multiplicando as matrizes:

$$
\begin{gathered}
{\left[\begin{array}{cc}
\sum_{t=1}^{n} \widehat{\sigma}_{v v t t}^{-1}\left[\bar{X}_{t .}^{2}-\left(1-\delta n^{-1}\right) S_{u u t t}\right] & -\sum_{t=1}^{n} \widehat{\sigma}_{v v t t}^{-1} \bar{X}_{t .} \\
-\sum_{t=1}^{n} \widehat{\sigma}_{v v t t}^{-1} \bar{X}_{t .} & \sum_{t=1}^{n} \widehat{\sigma}_{v v t t}^{-1}
\end{array}\right] \times\left[\begin{array}{c}
\sum_{t=1}^{n} \widehat{\sigma}_{v v t t}^{-1} Y_{t} \\
\sum_{t=1}^{n} \widehat{\sigma}_{v v t t}^{-1} \bar{X}_{t .} Y_{t}
\end{array}\right]=} \\
{\left[\begin{array}{c}
\left\{\sum_{t=1}^{n} \widehat{\sigma}_{v v t t}^{-1}\left[\bar{X}_{t .}^{2}-\left(1-\delta n^{-1}\right) S_{u u t t}\right]\right\}\left(\sum_{t=1}^{n} \hat{\sigma}_{v v t t}^{-1} Y_{t}\right)-\left(\sum_{t=1}^{n} \widehat{\sigma}_{v v t t}^{-1} \bar{X}_{t .}\right)\left(\sum_{t=1}^{n} \widehat{\sigma}_{v v t t}^{-1} \bar{X}_{t} Y_{t}\right) \\
-\left(\sum_{t=1}^{n} \widehat{\sigma}_{v v t t}^{-1} \bar{X}_{t .}\right)\left(\sum_{t=1}^{n} \widehat{\sigma}_{v v t t}^{-1} Y_{t}\right)^{n}+\left(\sum_{t=1}^{n} \widehat{\sigma}_{v v t t}^{-1}\right)\left(\sum_{t=1}^{n} \widehat{\sigma}_{v v t t}^{-1} \bar{X}_{t .} Y_{t}\right)
\end{array}\right] .}
\end{gathered}
$$


Mas

$$
\begin{aligned}
& \left.\qquad\left(\sum_{t=1}^{n} \widehat{\sigma}_{v v t t}^{-1}\right)\left(\sum_{t=1}^{n} \widehat{\sigma}_{v v t t}^{-1}\left[\bar{X}_{t .}^{2}-\left(1-\delta n^{-1}\right) S_{u u t t}\right]\right)-\left(\sum_{t=1}^{n} \widehat{\sigma}_{v v t t}^{-1} \bar{X}_{t .}\right)^{2}\right\}= \\
& =\left(\sum_{t=1}^{n} \widehat{\sigma}_{v v t t}^{-1}\right)\left\{\sum_{t=1}^{n}\left[\widehat{\sigma}_{v v t t}^{-1}\left(\bar{X}_{t .}-\bar{X}_{v v . .}^{*}\right)^{2}\right]-\left(1-\delta n^{-1}\right)\left(\sum_{t=1}^{n} \frac{\widehat{\sigma}_{v v t t}^{-1}}{r_{t}}\right) S_{u u}\right\}, \\
& \text { em que } \bar{X}_{v v . .}^{*}=\frac{\sum_{t=1}^{n}\left(\widehat{\sigma}_{v v t t}^{-1} \bar{X}_{t .}\right)}{\sum_{t=1}^{n} \widehat{\sigma}_{v v t t}^{-1}} .
\end{aligned}
$$

Simplificando o produto das matrizes (3.89), temos a seguinte matriz:

$$
\left[\begin{array}{c}
\left(\sum_{t=1}^{n} \widehat{\sigma}_{v v t t}^{-1}\left[\bar{X}_{t .}^{2}-\left(1-\delta n^{-1}\right) S_{u u t t}\right]\right)\left(\sum_{t=1}^{n} \widehat{\sigma}_{v v t t}^{-1} Y_{t}\right)-\left(\sum_{t=1}^{n} \widehat{\sigma}_{v v t t}^{-1} \bar{X}_{t .}\right)\left(\sum_{t=1}^{n} \widehat{\sigma}_{v v t t}^{-1} \bar{X}_{t .} Y_{t}\right) \\
\left(\sum_{t=1}^{n} \widehat{\sigma}_{v v t t}^{-1}\right)\left\{\sum_{t=1}^{n} \widehat{\sigma}_{v v t t}^{-1}\left(\bar{X}_{t .}-\bar{X}_{v v . .}^{*}\right)\left(\bar{Y}_{t}-\bar{Y}_{v v . .}^{*}\right)\right\}
\end{array}\right] .
$$

De (3.90) e (3.91), temos

$$
\begin{gathered}
\widehat{\beta}_{b w}=\frac{\left(\sum_{t=1}^{n} \widehat{\sigma}_{v v t t}^{-1}\right)^{-1} \sum_{t=1}^{n} \sum_{t=1}^{n} \widehat{\sigma}_{v v t t}^{-1}\left(\bar{X}_{t .}-\bar{X}_{v v . .}^{*}\right)\left(Y_{t}-\bar{Y}_{v v .}\right)}{\left(\sum_{t=1}^{n} \widehat{\sigma}_{v v t t}^{-1}\right)^{-1}\left[\sum_{t=1}^{n} \widehat{\sigma}_{v v t t}^{-1}\left(\bar{X}_{t .}-\bar{X}_{v v . .}^{*}\right)^{2}-\left(1-\delta n^{-1}\right)\left(\sum_{t=1}^{n} \frac{\widehat{\sigma}_{v v t t}^{-1}}{r_{t}}\right) S_{u u}\right]} \\
=\frac{m_{\bar{X} Y v v}}{m_{\bar{X} \bar{X} v v}-\left(1-\delta n^{-1}\right)\left[\frac{\left.\sum_{t=1}^{n} \frac{\hat{\sigma}_{v v t t}^{-1}}{r_{t}^{n}}\right] S_{t=1} \widehat{\sigma}_{v v t t}^{-1}}{\sum_{u u}}\right.}
\end{gathered}
$$

$\mathrm{e}$

$$
\widehat{\alpha}_{b w}=\bar{Y}_{v v .}-\widehat{\beta}_{b w} \bar{X}_{v v . .}^{*}
$$

em que $\bar{Y}_{v v .}^{*}=\frac{\sum_{t=1}^{n}\left(\widehat{\sigma}_{v v t t}^{-1} Y_{t}\right)}{\sum_{t=1}^{n} \widehat{\sigma}_{v v t t}^{-1}}$. Para $\hat{\lambda} \leq 1+n^{-1}$, substituir $\left(1-\delta n^{-1}\right)$ por $\left(\hat{\lambda}-n^{-1}-\delta n^{-1}\right)$ na equação (3.92), sendo $\hat{\lambda}$ a menor raíz de

$$
\left|\sum_{t=1}^{n} \widehat{\sigma}_{v v t t}^{-1}\left(\mathbf{Z}_{t}^{\prime} \mathbf{Z}_{t}-\lambda \mathbf{S}_{\text {aatt }}\right)\right|=0
$$

Analogamente ao caso precedente, temos que

$$
\widehat{\lambda}=\left[\left(\frac{\sum_{t=1}^{n} \frac{\widehat{\sigma}_{v v t t}^{-1}}{r_{t}}}{\sum_{t=1}^{n} \widehat{\sigma}_{v v t t}^{-1}}\right) S_{u u}\right]^{-1}\left(m_{\bar{X} \bar{X} v v}-\frac{m_{Y \bar{X} v v}^{2}}{m_{Y Y v v}}\right) .
$$


Os valores adotados para $\delta$, conforme as explicações anteriores, são:

$$
\delta=2, \quad \delta=5 \quad \text { ou } \quad \delta=1+\left(m_{\bar{X} \bar{X} v v}\right)^{-1} S_{u u v v}
$$

com

$$
m_{\bar{X} \bar{X} v v}=\frac{\sum_{t=1}^{n} \widehat{\sigma}_{v v t t}^{-1}\left(\bar{X}_{t .}-\bar{X}_{v v . .}^{*}\right)^{2}}{\sum_{t=1}^{n} \widehat{\sigma}_{v v t t}^{-1}}, \quad S_{u u v v}=\left(\frac{\sum_{t=1}^{n} \frac{\widehat{\sigma}_{v v t t}^{-1}}{r_{t}}}{\sum_{t=1}^{n} \widehat{\sigma}_{v v t t}^{-1}}\right) S_{u u},
$$

$\hat{\sigma}_{v v t t}=S_{\text {eett }}+\hat{\beta}_{N B 3}^{2}\left(\frac{S_{u u}}{r_{t}}\right)$. Observe que o estimador $\hat{\beta}_{N B 3}^{2}$ é utilizado como um estimador preliminar.

\section{Estimador $\hat{\beta}_{w}$}

A segunda versão do estimador de $\beta$ com os pesos "melhorados" considera um caso prático importante, a saber quando uma parte dos recursos da pesquisa é usada para determinações duplas num subconjunto da amostra. Nesta situação, a variância do erro de mensuração varia de acordo com o número de replicações, e os pesos são construídos pelas estimativas das matrizes de covariâncias das distribuições limites dos estimadores dos momentos das verdadeiras variáveis, isto é, dos seguintes estimadores das matrizes $M_{x y}$ e $M_{x x}$ :

$$
\hat{M}_{x y}=n^{-1}\left[\begin{array}{c}
\sum_{t=1}^{n} Y_{t} \\
\sum_{t=1}^{n}\left(\bar{X}_{t} Y_{t}\right)
\end{array}\right]
$$

$\mathrm{e}$

$$
\begin{aligned}
\hat{M}_{x x} & =n^{-1}\left[\left(\begin{array}{cc}
n & \sum_{t=1}^{n} \bar{X}_{t .} \\
\sum_{t=1}^{n} \bar{X}_{t .} & \sum_{t=1}^{n} \bar{X}_{t .}^{2}
\end{array}\right)-\sum_{t=1}^{n}\left(\begin{array}{cc}
0 & 0 \\
0 & S_{u u t t}
\end{array}\right)\right] \\
& =n^{-1}\left[\begin{array}{cc}
n & \sum_{t=1}^{n} \bar{X}_{t .} \\
\sum_{t=1}^{n} \bar{X}_{t .} & \sum_{t=1}^{n}\left(\bar{X}_{t .}-S_{u u t t}\right)
\end{array}\right] .
\end{aligned}
$$

A utilização da letra maíscula para denotar momentos indica que a soma total dos quadrados não é corrigida pela média. Estes são estimadores não-viciados de $M_{x y} \mathrm{e}$ $M_{x x}$, e são os utilizados no Teorema 2.2.1 em Fuller (1987). O estimador preliminar 
utilizado é, novamente, $\hat{\beta}_{N B 3}$, que é uma adaptação do estimador dado pela equação (3.1.33) em Fuller (1987) e pode ser escrita como

$$
\tilde{\beta}_{N B 3}=\hat{M}_{x x}^{-1} \hat{M}_{x y}, \quad S_{u u t t}=\left(\frac{1}{r_{t}}\right) S_{u u}
$$

Para estimar os parâmetros do modelo em questão, uma amostra de tamanho $n$ é selecionada de uma população e determinações duplas, i.i.d's, são realizadas sobre um subconjunto de " $d_{f}$ " indivíduos dentre os $n$ da amostra. Estas determinações i.i.d's são utilizadas para estimar a variância do erro de mensuração, $\boldsymbol{\Sigma}_{u u}$, supondo-a desconhecida. Este estimador já foi analisado anteriormente. Para o caso geral, ou seja, replicações das duas variáveis, resposta e explanatória, teremos o estimador de matriz de covariâncias dos erros $e_{t}$ e $u_{t}$, ou seja, da matriz $\Sigma_{a a}$, que denotaremos por $\mathrm{S}_{a a}$, cuja expressão é

$$
\mathrm{S}_{a a}=\frac{\sum_{t=1}^{n} \sum_{j=1}^{r_{t}}\left(\mathbf{Z}_{t j}-\mathbf{Z}_{t .}\right)^{\prime}\left(\mathbf{Z}_{t j}-\overline{\mathbf{Z}}_{t .}\right)}{\sum_{t=1}^{n}\left(r_{t}-1\right)}
$$

com

$$
\begin{gathered}
\mathrm{Z}_{t j}=\left(Y_{t j}, \mathbf{X}_{t j}^{\prime}\right)^{\prime}=\left(y_{t}+e_{t j}, 1, x_{t}+u_{t j}\right)^{\prime}, \\
\mathbf{a}_{t j}=\left(e_{t j}, 0, u_{t j}\right)^{\prime}, \\
\operatorname{Var}\left(\mathbf{a}_{t 1}, \mathbf{a}_{t 2}\right)=\text { bloco diagonal }\left(\Sigma_{a a}, \Sigma_{a a}\right) .
\end{gathered}
$$

Observe-se que a amostra inicial foi dividida em duas partes. A primeira subamostra com $d_{f}$ observações duplicadas iid 's; e a segunda com $\left(n-d_{f}\right)$ observações iid 's simples. Assim, todos os momentos, estimadores e outros resultados amostrais referentes à primeira subamostra foram indexados por "11", e aos referentes à segunda por "22". O estimador $\hat{\beta}_{N B 3}$, que aqui é uma adaptacão que em (Fuller 1987) aparece como equação (3.1.33), será utilizado nos cálculos das estimativas de variâncias das 
distribuiçoes limites de $\hat{\mathbf{M}}_{z z}$. Podemos interpretá-lo como uma média ponderada simples dos estimadores contruídos das primeiras " $d_{f}$ " observações (duplas) e das últimas $\left(n-d_{f}\right)$ observações; logo não é eficiente, conforme Fuller (1987), pois pesos simples não são pesos ótimos. A proposta, então, é construir uma nova média ponderada dos estimadores $\hat{\beta}_{N B 3_{11}}$ e $\hat{\beta}_{N B 3_{22}}$, com pesos que incorporam de forma mais ampla as informações oriundas das replicações. A seguir apresentamos a construção desses pesos ótimos, que na verdade, são adaptações dos pesos apresentados em Fuller (1987, eq. 3.1.19).

Se apenas as primeiras $d_{f}$ observações (duplas determinações i.i.d's) são utilizadas para estimar $\beta$, a variáncia da distribuição limite de $\widehat{\beta}_{N B 3_{11}}$ (ver Fuller, 1987 teor. 2.21) é

$$
\begin{aligned}
V_{\beta \beta_{11}}= & \left(d_{f}\right)^{-1} \Sigma_{x x}^{-1}\left(\Sigma_{x x} \sigma_{v v_{11}}+\Sigma_{\bar{u} \bar{u}} \sigma_{v v_{11}}+\Sigma_{\bar{u} v} \Sigma_{v \bar{u}}\right) \Sigma_{x x}^{-1} \\
& +\left(d_{f}\right)^{-1} \Sigma_{x x}^{-1}\left(\Sigma_{\bar{u} \bar{u}} \sigma_{\bar{r} \bar{r}}+\Sigma_{\bar{u} v} \Sigma_{v \bar{u}}\right) \Sigma_{x x}^{-1}
\end{aligned}
$$

com

$$
\begin{gathered}
\bar{u}_{t .}=\frac{\sum_{j=1}^{r_{t}} u_{t j}}{r_{t}}, \\
\Sigma_{\bar{u} \bar{u}}=\operatorname{Var}\left(\overline{\mathbf{u}}_{\mathrm{t} .}\right)=\frac{1}{r_{t}} \Sigma_{u u}=\frac{1}{2}\left[\begin{array}{cc}
0 & 0 \\
0 & \sigma_{u u}
\end{array}\right], \\
\Sigma_{\bar{u} v}=\operatorname{Cov}\left(\bar{u}_{t .}, v_{t}\right)=-\Sigma_{\bar{u} v} \beta=-\frac{1}{2} \Sigma_{u u} \beta=\frac{\Sigma_{u v}}{2}=-\frac{1}{2}\left[\begin{array}{c}
0 \\
\beta \sigma_{u u}
\end{array}\right]
\end{gathered}
$$

e

$$
r_{t j}=-u_{t j} \beta, \bar{r}_{t}=-\left(\bar{u}_{t .}\right) \beta,, \Longrightarrow \sigma_{\bar{r} \bar{r}}=\frac{\beta^{2} \sigma_{u u}}{2}=\frac{\sigma_{r r}}{2} .
$$

Portanto, temos

$$
\begin{aligned}
V_{\beta \beta_{11}}= & \left(d_{f}\right)^{-1} \Sigma_{x x}^{-1}\left(\Sigma_{x x} \sigma_{v v_{11}}+\frac{1}{2} \Sigma_{u u} \sigma_{v v_{11}}+\frac{1}{4} \Sigma_{u v} \Sigma_{v u}\right) \Sigma_{x x}^{-1} \\
& +\left(d_{f}\right)^{-1} \Sigma_{x x}^{-1}\left(\frac{1}{4} \Sigma_{u u} \sigma_{r r}+\frac{1}{4} \Sigma_{u v} \Sigma_{v u}\right) \Sigma_{x x}^{-1}
\end{aligned}
$$


Da expressão de $V_{\beta \beta_{11}}$ a variância da distribuição limite de $\hat{M}_{x y_{11}}$ é:

$$
V\left(\hat{M}_{x y_{11}}\right)=V_{M M_{11}}=\Sigma_{x x} V_{\beta \beta_{11}} \Sigma_{x x}
$$

pois, segundo Fuller (1987), $\hat{M}_{x y_{11}}=\Sigma_{x x} \widehat{\beta}_{N B 3_{11}}+O_{p}\left(n^{-1}\right)$.

Agora, se as últimas $\left(n-d_{f}\right)$ observações são usadas para estimar $\beta$, a variáncia de distribuição limite do estimador $\widehat{\boldsymbol{\beta}}_{N B 3_{22}}$ é:

$$
\begin{aligned}
V_{\beta \beta_{22}}= & \left(n-d_{f}\right)^{-1} \Sigma_{x x}^{-1}\left(\Sigma_{x x} \sigma_{v v_{22}}+\Sigma_{u u} \sigma_{v v_{22}}+\Sigma_{u v} \Sigma_{v u}\right) \Sigma_{x x}^{-1} \\
& +\left(d_{f}\right)^{-1} \Sigma_{x x}^{-1}\left(\Sigma_{u u} \sigma_{r r}+\Sigma_{u v} \Sigma_{v u}\right) \Sigma_{x x}^{-1}
\end{aligned}
$$

onde

$$
\sigma_{v v_{22}}=\sigma_{e e}+\sigma_{r r}
$$

A variância da distribuição aproximada de $\hat{M}_{x y_{22}}$ é, então,

$$
V_{M M_{22}}=\Sigma_{x x} V_{\beta \beta_{22}} \Sigma_{x x}
$$

pois $\hat{M}_{x y_{22}}=\Sigma_{x x} \widehat{\beta}_{N B 3_{22}}+O_{p}\left(n^{-1}\right)$.

Ainda, de acordo com Fuller (1987), como o mesmo estimador $\left(\mathbf{S}_{a a}\right)$ da matriz de covariância do erro de mensuração $\left(\Sigma_{a a}\right)$ é usada nos dois estimadores de $M_{x y}$ a covariância da distribuição conjunta assintótica de $\hat{M}_{x y_{11}}$ e $\hat{M}_{x y_{22}}$ é

$$
V_{M M_{12}}=\left(2 d_{f}\right)^{-1}\left(\Sigma_{u u} \sigma_{r r}+\Sigma_{u v} \Sigma_{v u}\right)
$$

Com o estimador preliminar $\hat{\beta}_{N B 3}$ em (3.96) de $\beta$, podemos construir estimadores para todas as matrizes de covariâncias: $\mathrm{V}_{M M_{11}}, \mathrm{~V}_{M M_{12}}$ e $\mathrm{V}_{M M_{22}}$.

Os outros estimadores utilizados são

$$
\widehat{\Sigma}_{x x}=\hat{M}_{x x}=n^{-1}\left[\begin{array}{cc}
0 & \sum_{t=1}^{n} \bar{X}_{t .} \\
\sum_{t=1}^{n} \bar{X}_{t .} & \sum_{t=1}^{n}\left(\bar{X}_{t .}^{2}-\frac{S_{u u}}{r_{t}}\right)
\end{array}\right]
$$


em que

$$
\begin{gathered}
\bar{X}_{t .}=x_{t}+\bar{u}_{t .}=x_{t}+\frac{\sum_{j=1}^{r_{t}} u_{t j}}{r_{t}}, \\
Y_{t}=\alpha+\beta x_{t}+e_{t}=\alpha+\beta \bar{x}_{t .}+v_{t t}
\end{gathered}
$$

e $v_{t t}=e_{t}-\beta \bar{u}_{t .}$. De (3.105) temos, também, que

$$
v_{t t}=Y_{t}-\alpha-\beta \bar{X}_{t}
$$

Dado o estimador $\hat{\beta}_{N B 3}$ de $\beta$, temos que

$$
\begin{aligned}
\hat{v}_{t t} & =Y_{t}-\hat{\alpha}_{p}-\hat{\beta}_{N B 3} \bar{X}_{t .} \\
& =Y_{t}-\left(\bar{\alpha}_{.}-\hat{\beta} . \bar{X}_{. .}^{*}\right)-\hat{\beta}_{N B 3} \bar{X}_{t .} \\
& =\left(Y_{t}-\bar{Y}_{.}\right)-\left(\bar{X}_{t .}-\bar{X}_{. .}^{*}\right) \hat{\beta}_{N B 3} .
\end{aligned}
$$

Portanto, um estimador não-viciado de $\operatorname{Var}\left(v_{t t}\right)$, é

$$
\widehat{\operatorname{Var}}\left(v_{t t}\right)=(n-2)^{-1} \sum_{t=1}^{n}\left[\left(Y_{t}-\bar{Y}_{.}\right)-\left(\bar{X}_{t .}-\bar{X}_{. .}\right)\right]^{2}=\hat{\sigma}_{\bar{v} \bar{v}}
$$

Agora,

$$
\begin{aligned}
\operatorname{Var}\left(e_{t}\right) & =\sigma_{e e}=\operatorname{Var}\left(\beta \bar{u}_{t .}+v_{t t}\right) \\
& =\beta^{2} \frac{\sigma_{u u}}{r_{t}}+\operatorname{Var}\left(v_{t t}\right)+2 E\left(v_{t t} \beta \cdot \bar{u}_{t} .\right. \\
& =\beta^{2} \frac{\sigma_{u u}}{r_{t}}+\sigma_{v v t t}-2 \beta^{2} \frac{\sigma_{u u}}{r_{t}} \\
& =\sigma_{v v t t}-\beta^{2} \frac{\sigma_{u u}}{r_{t}} .
\end{aligned}
$$

Totalizando (3.106) sobre $t$, temos

$$
n \sigma_{e e}=\sum_{t=1}^{n} \sigma_{v v t t}-\beta^{2} \sigma_{u u}\left(\sum_{t=1}^{n} \frac{1}{r_{t}}\right)
$$

ou seja

$$
\sigma_{e e}=\sigma_{\bar{v} \bar{v}}-\beta^{2} \sigma_{u u}\left(\frac{\sum_{t=1}^{n} \frac{1}{r_{t}}}{n}\right)
$$


Portanto, um estimador para $\sigma_{e e}$ será

$$
\hat{\sigma}_{e e}=\hat{\sigma}_{\bar{v} \bar{v}}-\hat{\beta}_{N B 3}^{2} S_{u u}\left(\frac{\sum_{t=1}^{n} \frac{1}{r_{t}}}{n}\right) .
$$

Seja $r_{t}=-\beta u_{t}, \operatorname{logo} \operatorname{Var}\left(r_{t}\right)=\beta^{2} \sigma_{u u}=\sigma_{r r}$, e $\bar{r}_{t .}=-\beta \bar{u}_{t .} . \quad \operatorname{Assim}, \operatorname{Var}\left(\bar{r}_{t .}\right)=\sigma_{\bar{r} \bar{r}}=$ $\beta^{2} \sigma_{u u} / 2$.

De maneira similar, um estimador para $\sigma_{r r}$ é $\hat{\sigma}_{r r}=S_{r r}=\hat{\beta}_{N B 3}^{2} S_{u u}$.

Como $\sigma_{v v 22}=\sigma_{e e}+\sigma_{r r}$ e $\sigma_{v v 11}=\sigma_{e e}+\sigma_{\bar{r} \bar{r}}$, então

$\tilde{\sigma}_{v v 11}=\hat{\sigma}_{e e}+\frac{1}{2} \hat{\beta}_{N B 3}^{2} S_{u u}$ e $\tilde{\sigma}_{v v 22}=\hat{\sigma}_{e e}+\hat{\beta}_{N B 3}^{2} S_{u u}$.

Também, como

$$
\Sigma_{u u}=\left[\begin{array}{cc}
0 & 0 \\
0 & \sigma_{u u}
\end{array}\right] \Longrightarrow \widehat{\Sigma}_{u u}=\left[\begin{array}{cc}
0 & 0 \\
0 & S_{u u}
\end{array}\right]
$$

e

$$
\Sigma_{u v}=-\Sigma_{u u} \beta \Longrightarrow \Sigma_{u v}=-\left[0, \sigma_{u u} \beta\right] \Longrightarrow \widehat{\Sigma}_{u v}=-\left[0, S_{u u} \hat{\beta}_{N B 3}\right] .
$$

Com os estimadores acima podemos construir estimadores para todas as matrizes $\mathrm{V}_{M M_{11}}, \mathrm{~V}_{M M_{22}}$ e $\mathrm{V}_{M M_{12}}=\mathrm{V}_{M M_{21}}$, que constituirão os pesos "melhorados". Assim, temos

$$
\tilde{\mathbf{V}}_{M M}=\left[\begin{array}{cc}
\tilde{\mathbf{V}}_{M M 11} & \tilde{\mathbf{V}}_{M M 12} \\
\tilde{\mathbf{V}}_{M M 21} & \tilde{\mathbf{V}}_{M M 22}
\end{array}\right] .
$$

Então, a segunda versão ponderada do estimador "melhorado"de $\beta$, será

$$
\begin{aligned}
\hat{\beta}= & {\left[\left(\hat{\mathrm{M}}_{x x 11}, \hat{\mathrm{M}}_{x x 22}\right) \tilde{\mathrm{V}}_{M M^{-1}}\left(\hat{\mathrm{M}}_{x x 11}, \hat{\mathrm{M}}_{x x 22}\right)\right]^{-1} } \\
& \times\left[\left(\hat{\mathrm{M}}_{x x 11}, \hat{\mathrm{M}}_{x x 22}\right) \tilde{\mathrm{V}}_{M M}^{-1}\left(\hat{\mathrm{M}}_{x y 11}^{\prime}, \hat{\mathrm{M}}_{x y 122}^{\prime}\right)^{\prime}\right]
\end{aligned}
$$

em que

$$
\hat{\mathrm{M}}_{z z j j}= \begin{cases}\mathrm{M}_{Z Z 11}-\frac{1}{2} \mathrm{~S}_{a a}, & \text { para } j=1, \\ \mathrm{M}_{Z Z 22}-\mathrm{S}_{a a}, & \text { para } j=2,\end{cases}
$$


sendo $\mathrm{a}_{t j}=\left(e_{t}, 0, u_{t j}\right), \mathrm{e}$

$$
\mathrm{S}_{a a}=\left[\begin{array}{ccc}
0 & 0 & 0 \\
0 & 0 & 0 \\
0 & 0 & S_{u u}
\end{array}\right]=\left[\begin{array}{cc}
0 & 0 \\
\mathbf{0}^{\prime} & \mathrm{S}_{u u}
\end{array}\right] .
$$

Portanto, podemos escrever

$$
\begin{gathered}
\hat{\mathbf{M}}_{x x 11}=\mathbf{M}_{\bar{X} \bar{X} 11}-\frac{1}{2} S_{u u} \\
\hat{\mathbf{M}}_{x x 22}=\mathbf{M}_{\bar{X} \bar{X} 22}-S_{u u} \\
\hat{\mathbf{M}}_{x y 11}=\mathbf{M}_{\bar{X} Y 11}, \\
\hat{M}_{x y 22}=M_{\bar{X} Y 22},
\end{gathered}
$$

sendo que

$$
\begin{aligned}
\hat{\mathbf{M}}_{z z 11} & =\mathrm{M}_{Z Z 11}-\frac{1}{2} S_{a a}=\left(d_{f}\right)^{-1} \sum_{t=1}^{d_{f}} \mathbf{Z}_{t 11}^{\prime} \mathrm{Z}_{t 11}-\frac{1}{2} \mathrm{~S}_{a a} \\
& =\left(d_{f}\right)^{-1} \sum_{t=1}^{d_{f}}\left(Y_{t 11}, 1, \bar{X}_{t .11}\right)^{\prime}\left(Y_{t 11}, 1, \bar{X}_{t .11}\right)-\frac{1}{2} \mathrm{~S}_{a a} \\
& =\left(d_{f}\right)^{-1} \sum_{t=1}^{d_{f}}\left[\begin{array}{ccc}
Y_{t}^{2} & Y_{t} & Y_{t} . \bar{X}_{t} \\
Y_{t} & 1 & \bar{X}_{t .} \\
\bar{X}_{t .} Y_{t} & \bar{X}_{t .} & \bar{X}_{t .}^{2}
\end{array}\right]-\frac{1}{2}\left[\begin{array}{ccc}
0 & 0 & 0 \\
0 & 0 & 0 \\
0 & 0 & S_{u u}
\end{array}\right] \\
& =\left[\begin{array}{ccc}
\frac{\sum_{t=1}^{d_{f}} Y_{t}^{2}}{d_{f}} & \frac{\sum_{t=1}^{n} Y_{t}}{d_{f}} & \frac{\sum_{t=1}^{d_{f}} Y_{t} \bar{X}_{t .}}{d_{f}} \\
\frac{\sum_{t=1}^{d_{f}} Y_{t}}{d_{f}} & 1 & \frac{\sum_{t=1}^{d_{f}} \bar{X}_{t .}}{d_{f}} \\
\frac{\sum_{t=1}^{d_{f}} \bar{X}_{t .}}{d_{f}} & \frac{\sum_{t=1}^{d_{f}} \bar{X}_{t . Y_{t}}}{d_{f}} & \frac{\sum_{t=1}^{d_{f}} \bar{X}_{t .}^{2}}{d_{f}}-\frac{1}{2} S_{u u}
\end{array}\right] \\
= & {\left[\begin{array}{lll}
\frac{\sum_{t=1}^{d_{f}} Y_{t}^{2}}{d_{f}} & \mathrm{M}_{Y \bar{X} 11} \\
\mathrm{M}_{\bar{X} \bar{X}_{11}} & \mathrm{M}_{\bar{X} \bar{X} 11} \bar{x}_{11}
\end{array}\right] . }
\end{aligned}
$$


Analogamente, temos

$\mathbf{M}_{z z 22}=\left[\begin{array}{ccc}\frac{\sum_{t=d_{f}+1}^{n} Y_{t}^{2}}{n-d_{f}} & \frac{\sum_{t=d_{f}+1}^{n} Y_{t}}{n-d_{f}} & \frac{\sum_{t=1}^{d_{f}} Y_{t} \bar{X}_{t .}}{n-d_{f}} \\ \frac{\sum_{t=d_{f}+1}^{n} Y_{t}}{n-d_{f}} & 1 & \frac{\sum_{t=d_{f}+1}^{n} \bar{X}_{t .}}{n-d_{f}} \\ \frac{\sum_{t=1}^{d_{f}} Y_{t} \bar{X}_{t .}}{n-d_{f}} & \frac{\sum_{t=d_{f}+1}^{n} \bar{X}_{t .}}{n-d_{f}} & \frac{\sum_{t=d_{f}+1}^{n} \bar{X}_{t .}^{2}}{n-d_{f}}-S_{u u}\end{array}\right]=\left[\begin{array}{cc}\frac{\sum_{t=d_{f}+1}^{n} Y_{t}^{2}}{n-d_{f}} & \mathbf{M}_{Y \bar{X} 22} \\ \mathbf{M}_{\bar{X} Y 22} & \mathbf{M}_{\bar{X} \bar{X} 22}\end{array}\right]$.

Um estimador da matriz de covariância da distribuição limite de $\hat{\beta}$, dado por Fuller (1987), é

$$
\widehat{V(\hat{\beta})}=\left[\left(\hat{M}_{x x 11}, \hat{M}_{x x 22}\right) \mathrm{V}_{M M}^{-1}\left(\hat{M}_{x x 11}, \hat{M}_{x x 22}\right)^{\prime}\right]^{-1}
$$

\subsection{Avaliações Numéricas}

Apresentamos, nesta Seção, os resultados de simulações comparando o comportamento dos estimadores alternativos de $\beta$, definidos nas Seções anteriores, com o EMV, para 2000 amostras com duplas replicações geradas pelos programas escritos na linguagem Ox, de acordo com o modelo dado em (2.1) e suposições (2.2).

Para a comparação do desempenho dos estimadores, calculou-se, além das duas já definidas (EMR e EQMR), as seguintes medidas, a saber:

(iii) Comprimento médio do Intervalo de Confiança de $95 \%$.

(iii) Taxa de sucessos, dada em porcentagem, do intervalo de $95 \%$ de confiança, definida por: $\left(n_{s} / n\right) 100 \%$, onde $n_{s}$ é o número de amostras nas quais o valor verdadeiro de $\beta$ (usado na simulação) pertence ao Intervalo de Confiança construído pelo estimador em questão.

(iv) ( $>$ limite superior do IC(95\%): Taxa de amostras, dada em porcentagem, nas quais o valor verdadeiro de $\beta$ supera o limite superior do Intervalo de $95 \%$.

(v) ( $<$ limite superior do IC(95\%): Taxa de amostras, dada em porcentagem, nas quais o valor verdadeiro de $\beta$ é inferior ao limite superior do Intervalo de $95 \%$. 
Cada simulação é composta por $n(n=100,1000)$ observações geradas de acordo com o modelo dado em (2.1) e suposições (2.2) e com replicações duplas sobre $\mathrm{r} \%(\mathrm{r}=5,20)$ das observações. Os valores dos parâmetros utilizados foram $\mu_{x}=68 ; \alpha=63 ; \beta=0,5 ; \sigma_{x x}=240 ; \sigma_{e e}=50$ e dois valores para $\sigma_{u u}, 60$ e 240.

$\mathrm{O}$ algoritmo EM utilizado para obter os EMV's foi considerado convergente quando a norma definida por $\sum_{i=1}^{6}\left|\left(\hat{\psi}_{i}^{p}-\hat{\psi}_{i}^{p+1}\right) / \psi_{i}\right|$ fosse inferior a $10^{-6}$ entre duas iterações consecutivas, onde $\psi_{i}, i=1, \ldots, 6$, representam os parâmetros do modelo.

Nas tabelas, a seguir, apresentamos os resultados das simulações.

Tabela 3.3 : Vícios, EQMRs empíricos e outras medidas $\sigma_{u u}=240 ;$ tamanho da amostra $100 ; 5 \%$ das observações da variável explanatória com replicações duplas.

\begin{tabular}{|c|c|c|c|c|c|}
\hline Estimadores & MQO & MV & $\hat{\beta}_{C}$ & $\hat{\beta}_{b w}$ & $\hat{\beta}_{w}$ \\
\hline Vícios Relativos (Vícios/ $\beta$ ) & $-0,4950$ & 0,1251 & $-0,0080$ & 0,0106 & $-0,2453$ \\
EQMO Relativo (EQMO/ $\beta^{2}$ ) & 0,2509 & 0,2899 & 0,1392 & 0,1613 & 0,2632 \\
Taxa de sucessos (\%) do IC(95\%) & & 80,13 & 78,3 & 66,65 & 73,8 \\
Comp. médio dos IC(95\%) & & 1,9858 & 2,6649 & 1,6079 & 0,9354 \\
Porcentagem de amostras para & & & & & \\
as quais o verdadeiro valor de $\beta$ & & & & & \\
$>$ limite superior do IC(95\%) & & 19,7 & 21,7 & 33,35 & 26 \\
<limite inferior do IC(95\%) & & 0 & 0 & 0 & 0,2 \\
\hline
\end{tabular}

Notações usadas: MV, máxima verossimilhança; $\hat{\beta}_{C}, \hat{\beta}_{N B 3}$ corrigido de vícios; $\widehat{\beta}_{b w}$, primeira versão do estimador ponderado melhorado; $\hat{\beta}_{w}$, segunda versão do estimador ponderado melhorado. 
Tabela 3.4 : Vícios, EQMRs empíricos e outras medidas $\sigma_{u u}=240 ;$ tamanho da amostra $100 ; 20 \%$ das observações da variável explanatória com replicações duplas.

\begin{tabular}{|c|c|c|c|c|c|}
\hline Estimadores & MQO & MV & $\hat{\beta}_{C}$ & $\hat{\beta}_{b w}$ & $\hat{\beta}_{w}$ \\
\hline Vícios Relativos (Vícios/ $\beta$ ) & $-0,4740$ & 0,1109 & 0,0066 & 0,0496 & $-0,1039$ \\
EQMO Relativo (EQM0/ $\beta^{2}$ ) & 0,2321 & 0,1784 & 0,0753 & 0,1246 & 0,0822 \\
Taxa de sucessos (\%) do IC(95\%) & & 90,6 & 88,75 & 82,0 & 86,1 \\
Comp. médio dos IC(95\%) & & 0,9220 & 1,0372 & 0,7308 & 0,6312 \\
Porcentagem de amostras para & & & & & \\
as quais o verdadeiro valor de $\beta$ & & & & & \\
$>$ limite superior do IC $(95 \%)$ & - & 9,4 & 11,25 & 17,85 & 13,95 \\
$<$ limite inferior do IC $(95 \%)$ & - & 0 & 0 & 0,15 & 0 \\
\hline
\end{tabular}

Tabela 3.5 : Vícios, EQMRs empíricos e outras medidas $\sigma_{u u}=240$, tamanho da amostra 1000; $5 \%$ das observações da variável explanatória com replicações duplas.

\begin{tabular}{|c|c|c|c|c|c|}
\hline Estimadores & MQO & MV & $\hat{\beta}_{C}$ & $\hat{\beta}_{b w}$ & $\hat{\beta}_{w}$ \\
\hline Vícios Relativos (Vícios/ $\beta$ ) & $-0,4931$ & 0,0371 & 0,0397 & 0,0411 & $-0,0335$ \\
EQMO Relativo (EQMO/ $\beta^{2}$ ) & 0,2438 & 0,0454 & 0,0550 & 0,0579 & 0,0323 \\
Taxa de sucessos (\%) do IC(95\%) & - & 92,1 & 91,15 & 51,15 & 89,4 \\
Comp. médio dos IC(95\%) & - & 0,4069 & 0,4932 & 0,1528 & 0,3842 \\
Porcentagem de amostras para & & & & & \\
as quais o verdadeiro valor de $\beta$ & & & & & \\
$>$ limite superior do IC(95\%) & - & 7,9 & 8,85 & 27,85 & 10,6 \\
$<$ limite inferior do IC(95\%) & - & 0 & 0 & 20,55 & 0 \\
\hline
\end{tabular}


Tabela 3.6 : Vícios, EQMRs empíricos e outras medidas $\sigma_{u u}=240$, tamanho da amostra 1000;20\% das observações da variável explanatória com replicações duplas.

\begin{tabular}{|c|c|c|c|c|c|}
\hline Estimadores & MQO & MV & $\hat{\beta}_{C}$ & $\hat{\beta}_{b w}$ & $\hat{\beta}_{w}$ \\
\hline Vícios Relativos (Vícios/ $\beta$ ) & $-0,4735$ & 0,0101 & 0,0103 & 0,0089 & $-0,0101$ \\
EQMO Relativo (EQMO/ $\beta^{2}$ ) & 0,2249 & 0,0111 & 0,0142 & 0,0134 & 0,0105 \\
Taxa de sucessos (\%) do IC(95\%) & - & 94,35 & 94,15 & 74,8 & 92,5 \\
Comp. médio dos IC(95\%) & - & 0,1973 & 0,2283 & 0,1273 & 0,1971 \\
Porcentagem de amostras para & & & & & \\
as quais o verdadeiro valor de $\beta$ & & & & & \\
$>$ limite superior do IC(95\%) & - & 5,05 & 5,85 & 15,3 & 7 \\
$<$ limite inferior do IC(95\%) & - & 0,6 & 0 & 9,9 & 0,5 \\
\hline
\end{tabular}

Tabela 3.7 : Vícios, EQMRs empíricos e outras medidas

$\sigma_{u u}=60$, tamanho da amostra $100 ; 5 \%$ das observações da variável explanatória com replicações duplas.

\begin{tabular}{|c|c|c|c|c|c|}
\hline Estimadores & MQO & MV & $\hat{\beta}_{C}$ & $\hat{\beta}_{b w}$ & $\hat{\beta}_{w}$ \\
\hline Vícios Relativos (Vícios/ $\beta$ ) & $-0,1953$ & 0,512 & 0,0287 & 0,0287 & $-0,0244$ \\
EQMO Relativo (EQMO/ $\beta^{2}$ ) & 0,0465 & 0,0739 & 0,0509 & 0,0515 & 0,0464 \\
Taxa de sucessos (\%) do IC(95\%) & - & 92,45 & 92,4 & 82,5 & 89,9 \\
Comp. médio dos IC(95\%) & - & 0,5148 & 0,5119 & 0,2666 & 0,4196 \\
Porcentagem de amostras para & & & & & \\
as quais o verdadeiro valor de $\beta$ & & & & & \\
$>$ limite superior do IC(95\%) & - & 7,55 & 7,6 & 10,5 & 9,5 \\
$<$ limite inferior do IC(95\%) & - & 0 & 0 & 7 & 0,6 \\
\hline
\end{tabular}


Tabela 3.8 : Vícios, EQMRs empíricos e outras medidas $\sigma_{u u}=60$, tamanho da amostra $100 ; 20 \%$ das observações da variável explanatória com replicações duplas.

\begin{tabular}{|c|c|c|c|c|c|}
\hline Estimadores & MQO & MV & $\hat{\beta}_{C}$ & $\hat{\beta}_{b w}$ & $\hat{\beta}_{w}$ \\
\hline Vícios Relativos (Vícios $/ \beta$ ) & $-0,4739$ & 0,1109 & 0,0066 & 0,0497 & $-0,1039$ \\
EQMO Relativo (EQMO/ $\beta^{2}$ ) & 0,2320 & 0,1783 & 0,0753 & $0,0,1246$ & 0,0822 \\
Taxa de sucessos (\%) do IC(95\%) & & 90,6 & 88,75 & 82 & 86,05 \\
Comp. médio dos IC(95\%) & - & 0,9220 & 1,0373 & 0,7308 & 0,6312 \\
Porcentagem de amostras para & & & & & \\
as quais o verdadeiro valor de $\beta$ & & & & & \\
$>$ limite superior do IC $(95 \%)$ & - & 9,4 & 11,25 & 17,85 & 13,95 \\
$<$ limite inferior do IC $(95 \%)$ & - & 0 & 0 & 0,15 & 0 \\
\hline
\end{tabular}

Tabela 3.9 : Vícios, EQMRs empíricos e outras medidas $\sigma_{u u}=60$, tamanho da amostra $1000 ; 5 \%$ das observações da variável explanatória com replicações duplas.

\begin{tabular}{|c|c|c|c|c|c|}
\hline Estimadores & MQO & MV & $\hat{\beta}_{C}$ & $\hat{\beta}_{b w}$ & $\hat{\beta}_{w}$ \\
\hline Vícios Relativos (Vícios $/ \beta$ ) & $-0,1960$ & 0,0031 & 0,0027 & 0,0027 & $-0,0006$ \\
EQMO Relativo (EQMO/ $\beta^{2}$ ) & 0,0393 & 0,0038 & 0,0039 & 0,0039 & 0,0036 \\
Taxa de sucessos (\%) do IC(95\%) & - & 95,75 & 95,4 & 79,15 & 95 \\
Comp. médio dos IC(95\%) & - & 0,1207 & 0,1238 & 0,0738 & 0,1207 \\
Porcentagem de amostras para & & & & & \\
as quais o verdadeiro valor de $\beta$ & & & & & \\
$>$ limite superior do IC(95\%) & - & 3,8 & 4,25 & 10,55 & 4,65 \\
$<$ limite inferior do IC(95\%) & - & 0,45 & 0,35 & 10,3 & 0,35 \\
\hline
\end{tabular}


Tabela 3.10 : Vícios, EQMRs empíricos e outras medidas $\sigma_{u u}=60$, tamanho da amostra $1000 ; 20 \%$ das observações da variável explanatória com replicações duplas.

\begin{tabular}{|c|c|c|c|c|c|}
\hline Estimadores & MQO & MV & $\hat{\beta}_{C}$ & $\hat{\beta}_{b w}$ & $\hat{\beta}_{w}$ \\
\hline Vícios Relativos (Vícios/ $\beta$ ) & $-0,1844$ & 0,0001 & $-0,0005$ & $-0,0005$ & $-0,0015$ \\
EQMO Relativo (EQMO/ $\beta^{2}$ ) & 0,0348 & 0,0019 & 0,0019 & 0,0019 & 0,0019 \\
Taxa de sucessos (\%) do IC(95\%) & - & 94,55 & 95,45 & 90,15 & 95,15 \\
Comp. médio dos IC(95\%) & - & 0,0831 & 0,0849 & 0,0719 & 0,0834 \\
Porcentagem de amostras para & & & & & \\
as quais o verdadeiro valor de $\beta$ & & & & & \\
$>$ limite superior do IC(95\%) & - & 3,6 & 3,35 & 5,7 & 3,85 \\
$<$ limite inferior do IC(95\%) & - & 1,85 & 1,2 & 4,15 & 2 \\
\hline
\end{tabular}

\section{Avaliação dos resultados de simulação}

As tendências nos resultados de simulação podem ser melhor analisadas observando, inicialmente, a situação simulada na qual a variância do erro de mensuração da variável explanatória denotada por $\sigma_{u u}$ é grande, ou seja, $\sigma_{u u}=240$. Isto equivale a $50 \%$ da variância da variável observada $X_{t j}, j=1, \ldots, r_{t}, t=1, \ldots, n$. Neste caso os desempenhos dos estimadores são bastantes semelhantes quando analisados pelas medidas propostas como: EQMRO, taxa de sucessos do intervalo de confiança de $95 \%$ e seu comprimento médio; ou seja, não há evidência de superioridade substancial de nenhum deles. Entretanto, com as condições mais favoráveis, por exemplo, para $\sigma_{u u}=60$ ( um quarto de $\sigma_{x x}$ ) e/ou $\mathrm{r}=20 \%$ de replicações, a superioridade relativa do EMV sobre os alternativos fica, em geral, mais evidente quando consideramos as mesmas medidas - Já com as condições plenamente favoráveis, ou seja, $n=1000,20 \%$ de duplicações e $\sigma_{u u}=60$, os comportamentos dos estimadores alternativos não são muitos inferiores ao da máxima verossimilhança. Em quase todos os casos verificou-se uma tendência do verdadeiro valor de $\beta$ cair acima do limite superior do intervalo de confiança, porém 
de forma menos acentuada para o EMV. Esta tendência tende a diminuir com algumas alterações, como, por exemplo:

(i) com a diminuição de $\sigma_{u u}$ relativamente a outras duas variâncias, particularmente a $\sigma_{x x}$, veja as tabelas a seguir.

Tabela 3.11 : Vícios, EQMRs empíricos e outras medidas $\sigma_{u u}=30, \sigma_{e e}=240, \sigma_{x x}=350$, tamanho da amostra $100 ; 20 \%$ das observações da variável explanatória com replicações duplas.

\begin{tabular}{|c|c|c|c|c|c|}
\hline Estimadores & MQO & MV & $\hat{\beta}_{C}$ & $\hat{\beta}_{b w}$ & $\hat{\beta}_{w}$ \\
\hline Taxa de sucessos (\%) do IC(95\%) & - & 94,55 & 95 & 94,4 & 93,95 \\
Porcentagem de amostras para & & & & & \\
as quais o verdadeiro valor de $\beta$ & & & & & \\
$>$ limite superior do IC(95\%) & - & 2,65 & 2,6 & 2,7 & 3,05 \\
$<$ limite inferior do IC(95\%) & - & 2,8 & 2,4 & 2,9 & 3 \\
\hline
\end{tabular}

Tabela 3.12 : Vícios, EQMRs empíricos e outras medidas $\sigma_{u u}=20, \sigma_{e e}=200, \sigma_{x x}=240$, tamanho da amostra 100;20\% das observações da variável explanatória com replicações duplas.

\begin{tabular}{|c|c|c|c|c|c|}
\hline Estimadores & MQO & MV & $\hat{\beta}_{C}$ & $\hat{\beta}_{b w}$ & $\hat{\beta}_{w}$ \\
\hline Taxa de sucessos (\%) do IC(95\%) & - & 94,45 & 94.85 & 94,4 & 93,8 \\
Porcentagem de amostras para & & & & & \\
as quais o verdadeiro valor de $\beta$ & & & & & \\
$>$ limite superior do IC(95\%) & - & 2,65 & 2,65 & 2,65 & 2,95 \\
< limite inferior do IC(95\%) & - & 2,9 & 2,5 & 2,95 & 3.25 \\
\hline
\end{tabular}

(ii) com o aumento de $\mathrm{r}(\%)$, isto é, aumento de porcentagem das observações duplicadas de variável explanatória, conforme as duas tabelas a seguir, baseadas em 2000 amostras simuladas. 
Tabela 3.13 : Vícios, EQMRs empíricos e outras medidas $\sigma_{u u}=30, \sigma_{e e}=240, \sigma_{x x}=240$, tamanho da amostra $100 ; 20 \%$ das observações da variável explanatória com replicações duplas.

\begin{tabular}{|c|c|c|c|c|c|}
\hline Estimadores & MQO & MV & $\hat{\beta}_{C}$ & $\hat{\beta}_{b w}$ & $\hat{\beta}_{w}$ \\
\hline Taxa de sucessos (\%) do IC(95\%) & - & 95 & 95.3 & 94,5 & 93,95 \\
Porcentagem de amostras para & & & & & \\
as quais o verdadeiro valor de $\beta$ & & & & & \\
$>$ limite superior do IC(95\%) & - & 2,8 & 2,65 & 2,75 & 3,15 \\
$<$ limite inferior do IC(95\%) & - & 2,2 & 2,05 & 2,75 & 2,9 \\
\hline
\end{tabular}

Tabela 3.14 : Vícios, EQMRs empíricos e outras medidas $\sigma_{u u}=30, \sigma_{e e}=240, \quad \sigma_{x x}=240$, tamanho da amostra 100 e $50 \%$ das observações da variável explanatória com replicações duplas.

\begin{tabular}{|c|c|c|c|c|c|}
\hline Estimadores & MQO & MV & $\hat{\beta}_{C}$ & $\hat{\beta}_{b w}$ & $\hat{\beta}_{w}$ \\
\hline Taxa de sucessos (\%) do IC(95\%) & - & 94,95 & 95.25 & 95 & 94,95 \\
Porcentagem de amostras para & & & & & \\
as quais o verdadeiro valor de $\beta$ & & & & & \\
$>$ limite superior do IC(95\%) & - & 2,5 & 2,45 & 2,55 & 2,65 \\
<limite inferior do IC(95\%) & - & 2,55 & 2,3 & 2,45 & 2,4 \\
\hline
\end{tabular}

O estimador de mínimos quadrados ordinário denotado por $M Q O$ foi incluído para mostrar a redução de vícios obtida pelos estimadores alternativos construídos a partir do método dos momentos. 


\section{Capítulo 4}

\section{Relação Linear Funcional com Replicações}

\subsection{Introdução}

Neste capítulo investigaremos a estimação de máxima verossimilhança dos parâmetros estruturais nas relações lineares funcionais com replicações. Esta abordagem é baseada em Mak (1982), onde resultados assintóticos gerais para estimação de máxima verossimilhanca, na presença de parâmetros incidentais, são estabelecidos.

Estimação de parâmetros estruturais, quando o número de parâmetros desconhecidos aumenta com o tamanho da amostra, apresenta alguns problemas: os estimadores de máxima verossimilhança podem ser inconsistentes (Neyman e Scott, 1948); a matriz de covariância assintótica desses estimadores não coincide com o inverso da matriz de informação (Patefield, 1977 e 1978); sob a normalidade dos erros de mensurações, o logaritmo da função de verossimilhança é ilimitado e o uso do método de máxima verossimilhança é, à primeira vista, injustificável; a raiz das equações da primeira derivada parcial com respeito aos parâmetros estruturais é um ponto de sela e não de máximo (Solari, 1969); a não existência do (vetor) máximo do logaritmo da função de verossimilhança no espaço paramétrico, ou mais especificamente, o valor de $\beta$ que maximiza tal função não pertence à região admissível do espaço paramétrico (para mais detalhes 
veja, por exemplo, Vilca-Labra (1996).

Neste contexto, são necessárias suposições adicionais, que tornam factível o estudo de inferência sob normalidade. Dentre elas as mais comumente adotadas:

(i) $\sigma_{e e}$ e $\sigma_{u u}$ conhecidos (Barnet, 1969, obtém estimadores de máxima verossimilhança de forma fechada).

(ii) $\sigma_{e e}$ ou $\sigma_{u u}$ conhecido (veja Cheng e Van Ness, 1991, entre outros).

(iii) $\lambda=\sigma_{e e} / \sigma_{u u}$ conhecida (Lindley e El Sayad, 1968, Zellner, 1971, Kendal e Stuart, 1979, Glesser, 1981, entre outros).

Contudo, Mak (1982), sob um modelo bem geral, estabeleceu certas condições gerais para garantir a consistência e a distribuição assintótica dos estimadores dos parâmetros estruturais (que incluem em particular o EMV) obtidos via abordagem de verossimilhança usual, quando os parâmetros incidentais são primeiramente substituídos pelas suas estimativas (que podem depender de parâmetros estruturais). Essas condições foram aplicadas por Mak (1982), entre outros, ao modelo funcional univariado definido pela relação linear:

$$
y_{t}=\alpha+\beta x_{t}
$$

onde $x_{1}, \ldots, x_{n}$ são quantidades não aleatórias ou parâmetros incidentais, $\alpha$ e $\beta$ são parâmetros estruturais desconhecidos. Os pares $\left(x_{t}, y_{t}\right)$ são os verdadeiros valores das variáveis explanatórias e respostas não observáveis (ou observados com erros), sendo que observamos os pares $\left(X_{t}, Y_{t}\right)$, definidos por:

$$
X_{t}=x_{t}+u_{t}, \quad Y_{t}=y_{t}+e_{t}, \quad t=1, \ldots, n,
$$

com

$$
\left(\begin{array}{l}
u_{t} \\
e_{t}
\end{array}\right) \sim \mathrm{N}_{2}\left[\left(\begin{array}{l}
0 \\
0
\end{array}\right),\left(\begin{array}{cc}
\sigma_{u u} & 0 \\
0 & \lambda \sigma_{u u}
\end{array}\right)\right],
$$

$t=1, \ldots, n$. Note que Mak (1982) usa a suposição (iii) para obter os estimadores de máxima verossimilhança dos parâmetros estruturais do modelo. 
Nosso objetivo é estudar a estimação de máxima verossimilhança considerando observações replicadas, que é uma maneira de garantir uma abordagem factível sem suposições adicionais sobre as variâncias dos erros. Assim, considerando $r_{t}$ e $s_{t}$ números de replicações sobre $x_{t}$ e $y_{t}$, respectivamente, reescrevemos o modelo acima como:

$$
X_{t i}=x_{t}+u_{t i} \quad e \quad Y_{t j}=y_{t}+e_{t j},
$$

com

$$
\left(\begin{array}{l}
u_{t i} \\
e_{t j}
\end{array}\right) \sim \mathrm{N}_{2}\left[\left(\begin{array}{l}
0 \\
0
\end{array}\right),\left(\begin{array}{cc}
\sigma_{u u} & 0 \\
0 & \sigma_{e e}
\end{array}\right)\right],
$$

$t=1, \ldots, n, \quad i=1, \ldots, r_{t}, \quad j=1, \ldots, s_{t}$.

Como essa abordagem permite a obtenção da matriz de covariância assintótica dos estimadores de máxima verossimilhança dos parâmetros estruturais do modelo é possível calcular as eficiências relativas assintóticas desses estimadores com respeito aos estimadores sugeridos em Dorf e Gurland (1960). A suposição de razão constante entre os números de replicações $\left(r_{t} / s_{t}\right)$ é necessária para estudar o comportamento assintótico dos EMVs, o que torna o modelo ligeiramente menos geral que o considerado por Dorf e Gurland (1960), no qual não há tal suposição. Contudo, mesmo na situação mais geral, podemos obter os estimadores de máxima verossimilhança ao implementar um algoritmo tipo EM, como considerado em Kimura (1992). No caso particular no qual $r_{t} / s_{t}=k, t=1, \ldots, n$, o EMV de $\beta$ é obtido ao resolver-se uma equação de quarto grau. Maiores simplicações ocorrem se $r_{t}=r$ e $s_{t}=s$ ou no caso em que $r_{t}=r$ e $s_{t}=1$, isto é, apenas $x_{t}$ é replicado, que constitui uma situação muito comum nos estudos práticos.

Na Seção 4.2 revisamos os resultados assintóticos estabelecidos por Mak (1982), com relação às condições de regularidade para a consistência e normalidade assintótica dos EMVs. Na Seção 4.3 estabelecemos as propriedades dos EMVs dos parâmetros incidentais e estruturais. Demonstramos que os estimadores de máxima verossimilhança de $\alpha$ e $\beta$ são consistentes, enquanto que os de $\sigma_{e e}$ e $\sigma_{u u}$ não são; entretanto é possível corrigir estes estimadores, tornando-os consistentes. Obtemos, também, a distribuição assintótica dos EMVs. A Seção 4.4 é dedicada a obtenção dos EMVs e seus estimadores consistentes modificados. 


\subsection{Notações e Resultados Preliminares}

Seja $\quad Z_{1}, \ldots, Z_{n}$ uma seqüência de vetores aleatórios independentes bidimensionais com função de log-verossimilhança dada por

$$
\sum_{t=1}^{n} \log f_{t}\left(z_{t} ; \theta, x_{t}\right)
$$

em que $f_{t}\left(z_{t} ; \theta, x_{t}\right)$ é a função densidade de $Z_{t}, t=1, \ldots, n ; \theta=\left(\theta_{1}, \ldots, \theta_{p}\right)^{\prime} \in \Theta \subset$ $\mathbf{R}^{p}$ e $x_{t} \in \mathbf{R}, t=1, \ldots, n$.

Supomos que $\theta^{0} \in \Theta$ e $x_{t}^{0} \in \chi_{\mathbf{t}}$, no qual $\theta^{\circ}$ e $x_{1}^{o}, \ldots, x_{n}^{o}$, denotam os valores paramétricos (estruturais e incidentais) verdadeiros. Os valores esperados e as variâncias são tomados em relação a $\theta^{\circ}$ e $x_{t}^{o}$ e são denotados por $E^{\circ}[]=.E[. \mid$ $\left.\theta^{o}, x_{1}^{o}, \ldots, x_{n}^{o}\right]$ e $V^{o}[]=.V\left[. \mid \theta^{o}, x_{1}^{o}, \ldots, x_{n}^{o}\right]$.

Para cada $\mathrm{t}$ e um dado $\theta$, supomos que exista $\tilde{x}_{t}=\tilde{x}_{t}\left(z_{t}, \theta\right)$ um estimador (dependente de $\theta$ ) de $x_{t}$, com possibilidade de ser um EMV condicional de $x_{t}$ dado $\theta$, obtido ao maximizar-se (4.6) em relação a $x_{t}$, para $\theta$ fixo.

Agora, substituindo $x_{t}$ por $\tilde{x}_{t}$ em (4.6), obtemos

$$
\sum_{t=1}^{n} \log f_{t}\left[z_{t} ;\left(\theta, \tilde{x}_{t}\right)\right]=\sum_{t=1}^{n} h_{t}\left(z_{t} ; \theta\right) .
$$

Vamos definir as seguintes funções, usando as notações sugeridas por Mak (1982) e admitindo que as derivadas existem q.c.:

$$
\begin{aligned}
q_{t \theta_{j}}\left[z_{t}, \theta\right] & =\frac{\partial h_{t}\left(z_{t} ; \theta, \tilde{x}_{t}\right)}{\partial \theta_{j}}, \quad j=1, \ldots, p ; \\
q_{t \theta_{j} \theta_{k}}\left[z_{t}, \theta\right] & =\frac{\partial^{2} h_{t}\left(z_{t} ; \theta, \tilde{x}_{t}\right)}{\partial \theta_{k} \partial \theta_{j}}, \quad j, k=1, \ldots, p ; \\
q_{t \theta_{j}, \theta_{k}}\left[z_{t}, \theta\right] & =q_{t \theta_{j}}\left[z_{t}, \theta\right] q_{t \theta_{k}}\left[z_{t}, \theta\right], \quad j, k=1, \ldots, p .
\end{aligned}
$$

Além disso, defina $A_{n}(\theta)$ como a matriz de ordem p, simétrica e aleatória, com elemento $(\mathrm{j}, \mathrm{k})$ dado por

$$
a_{j k}=n^{-1} \cdot \sum_{t=1}^{n} q_{t \theta_{j} \theta_{k}}\left[z_{t}, \theta\right], \quad j, k=1, \ldots, p .
$$


Feitas estas considerações, Mak (1982) estuda as condições gerais de regularidade sob as quais (4.7) tem um máximo $\hat{\theta}_{n}=\tilde{\theta}\left(z_{1}, \ldots, z_{n}\right)$, que converge em probabilidade para algum $\theta^{1}$ no interior $\Theta^{\circ}$ de $\Theta$, onde $\theta^{1}$ é um máximo local da função

$$
\varphi(\theta)=n^{-1} \sum_{t=1}^{n} E^{o}\left\{h_{t}\left[Z_{t}, \theta\right]\right\}
$$

$\mathrm{e}$

$$
\sqrt{n}\left[V_{n}\left(\theta^{1}\right)\right]^{-\frac{1}{2}} E^{o}\left[A_{n}\left(\theta^{1}\right)\right]\left(\hat{\theta}_{n}-\theta^{1}\right) \stackrel{\mathrm{D}}{\longrightarrow} \mathrm{N}\left(0, \mathrm{I}_{p}\right),
$$

onde os elementos da matriz $V_{n}$ são dados por:

$$
v_{j k}=n^{-1} \sum_{t=1}^{n} \operatorname{Cov}^{o}\left(q_{t \theta_{j}}\left[Z_{t}, \theta\right], q_{t \theta_{k}}\left[Z_{t}, \theta\right]\right), \quad j, k=1, \ldots, p .
$$

Também, em algumas situações, é possível obter estimadores $\tilde{x}_{t}$, de tal forma que $\theta^{1}$ só dependa de $\theta^{\circ}$ (independente de $x_{t}^{o}$ ), isto é, existe uma função c(.), um a um, e $\theta^{1}=c\left(\theta^{\circ}\right)$. Assim, um estimador consistente de $\theta^{\circ}$, baseado em $\hat{\theta}_{n}$, pode ser construído, digamos, $\tilde{\theta}_{n}=c^{-1}\left(\hat{\theta}_{n}\right)$. Demonstração detalhada dos resultados são encontrados em Gimenez e Bolfarine (1997).

\subsection{Aplicação dos Resultados Anteriores ao Modelo Nor- mal Bivariado com Replicações.}

Nesta seção obtemos o estimador de máxima verossimilhança (EMV) de $x_{t}$ e mostramos que os EMVs dos verdadeiros parâmetros estruturais, $\theta^{o}=\left(\alpha^{o}, \beta^{o}, \sigma_{u u}^{o}, \sigma_{e e}^{o}\right)^{\prime}$, não são consistentes, isto é $\hat{\theta}_{n} \stackrel{\mathrm{P}}{\longrightarrow} \theta^{1} \neq \theta^{\circ}$. Especificamente, mostramos que $\tilde{\theta}_{n} \stackrel{\mathrm{P}}{\longrightarrow}$ $\theta^{\circ}=c^{-1}\left(\theta^{1}\right)$, como foi mencionado acima.

\subsubsection{A função de verossimilhança}

Sob o modelo (4.4), temos:

$$
\left(\begin{array}{c}
X_{t i} \\
Y_{t j}
\end{array}\right) \sim \mathrm{N}_{2}\left[\left(\begin{array}{c}
x_{t} \\
\alpha+\beta x_{t}
\end{array}\right),\left(\begin{array}{cc}
\sigma_{u u} & 0 \\
0 & \sigma_{e e}
\end{array}\right)\right] .
$$


Sejam

$$
\begin{aligned}
& \mathrm{X}_{t}=\left(X_{t 1}, X_{t 2}, \ldots, X_{t r_{t}}\right)^{\prime} \\
& \mathrm{Y}_{t}=\left(Y_{t 1}, Y_{t 2}, \ldots, Y_{t s_{t}}\right)^{\prime} \\
& \mathrm{Z}_{\mathrm{t}}=\left(\begin{array}{c}
\mathrm{X}_{\mathrm{t}} \\
\mathrm{Y}_{\mathrm{t}}
\end{array}\right) \sim \mathrm{N}_{\left(r_{t}+s_{t}\right)}\left(\mathrm{m}_{t}, \mathrm{~V}_{t}\right)
\end{aligned}
$$

em que

$$
\begin{aligned}
& \mathbf{m}_{t}=E\left(\mathbf{Z}_{t}\right)=\left(\begin{array}{c}
x_{t} \mathbf{1}_{r_{t} 1} \\
\left(\alpha+\beta x_{t}\right) \mathbf{1}_{s_{t} 1}
\end{array}\right), \\
& \mathbf{V}_{t}=\operatorname{Var}\left(\mathbf{Z}_{t}\right)=\left[\begin{array}{cc}
\sigma_{u u} \mathbf{I}_{r_{t}} & \mathbf{0}_{r_{t} r_{t}} \\
\mathbf{0}_{s_{t} r_{t}} & \sigma_{e e} \mathbf{I}_{s_{t}}
\end{array}\right] .
\end{aligned}
$$

Logo, temos

$$
\operatorname{det} \mathbf{V}_{t}=\sigma_{u u}^{r_{t}} \sigma_{r r}^{s_{t}}
$$

$\mathrm{e}$

$$
\mathbf{V}_{t}^{-1}=\left[\begin{array}{cc}
\sigma_{u u}^{-1} \mathbf{I}_{r_{t}} & \mathbf{0}_{r_{t} r_{t}} \\
\mathbf{0}_{s_{t} r_{t}} & \sigma_{e e}^{-1} \mathbf{I}_{s_{t}}
\end{array}\right]
$$

De (4.16) e (4.17), temos:

$$
\left(\mathbf{Z}_{t}-\mathbf{m}_{t}\right)^{\prime} \mathbf{V}^{-1}\left(\mathbf{Z}_{t}-\mathbf{m}_{t}\right)=\left[\sigma_{u u}^{-1} \sum_{i=1}^{r_{t}}\left(X_{t i}-x_{t}\right)^{2}+\sigma_{e e}^{-1} \sum_{j=1}^{s_{t}}\left(Y_{t j}-\alpha-\beta x_{t}\right)^{2}\right] .
$$

Então, dada uma amostra de tamanho $\mathrm{n}$ de $\mathrm{Z}_{t}$, obtemos a seguinte função de verossimilhança:

$$
f_{\mathbf{Z}}(\mathbf{z})=\prod_{t=1}^{n} f_{\mathbf{Z}_{t}}\left(\mathbf{z}_{t}\right)=\prod_{t=1}^{n}(2 \pi)^{-\left(\frac{\left(r_{t}+s_{t}\right)}{2}\right)}\left(\operatorname{det} \mathbf{V}_{t}\right)^{-\frac{1}{2}} \exp \left(-\frac{1}{2}\left[\left(\mathbf{z}_{t}-\mathbf{m}_{t}\right)^{\prime} \mathbf{V}_{t}^{-1}\left(\mathbf{z}_{t}-\mathbf{m}_{t}\right)\right]\right)
$$

em que $\mathrm{Z}=\left(\mathrm{Z}_{1}, \ldots, \mathrm{Z}_{n}\right)^{\prime}$. 
Substituindo (4.16), (4.17) e (4.18) em (4.19) segue-se que:

$$
\begin{aligned}
f_{\mathbf{Z}}(\mathbf{z})= & \prod_{t=1}^{n}(2 \pi)^{-\left(\frac{r_{t}+s_{t}}{2}\right)}\left(\sigma_{u u}^{r_{t}} \sigma_{e e}^{s_{t}}\right)^{-\frac{1}{2}} \exp \left\{-\frac{1}{2}\left[\sigma_{u u}^{-1} \sum_{i=1}^{r_{t}}\left(X_{t i}-x_{t}\right)^{2}\right.\right. \\
& \left.\left.+\sigma_{e e}^{-1} \sum_{j=1}^{s_{t}}\left(Y_{t j}-\alpha-\beta x_{t}\right)^{2}\right]\right\} .
\end{aligned}
$$

Portanto, a log-verossimilhança pode ser escrita como

$$
\begin{aligned}
l= & -\left(\frac{\sum_{t=1}^{n}\left(r_{t}+s_{t}\right)}{2}\right) \ln 2 \pi-\frac{\sum_{t=1}^{n} r_{t}}{2} \ln \sigma_{u u}-\frac{\sum_{t=1}^{n} s_{t}}{2} \ln \sigma_{e e}- \\
& -\frac{1}{2}\left(\sigma_{u u}^{-1} \sum_{t=1}^{n} \sum_{i=1}^{r_{t}}\left(X_{t i}-x_{t}\right)^{2}+\sigma_{e e}^{-1} \sum_{t=1}^{n} \sum_{j=1}^{s_{t}}\left(Y_{t j}-\alpha-\beta x_{t}\right)^{2}\right) .
\end{aligned}
$$

Lema 4.1 Dado $\theta=\left(\alpha, \beta, \sigma_{e e}, \sigma_{u u}\right)^{\prime}$, o EMV de $x_{t}$ é dado por:

$$
\tilde{x}_{t}=g_{t}\left[\left(\mathrm{X}_{t}, \mathrm{Y}_{t}\right) \mid \theta\right]=\frac{r_{t} \sigma_{u u}^{-1} \bar{X}_{t .}+s_{t} \beta \sigma_{e e}^{-1}\left(\bar{Y}_{t .}-\alpha\right)}{r_{t} \sigma_{u u}^{-1}+s_{t} \beta^{2} \sigma_{e e}^{-1}}
$$

\section{Prova}

A prova deste Lema segue diretamente da maximização da log-verossimilhança (4.21), mantendo $\theta$ fixo, ou mais especificamente, resolvendo a seguinte equação de estimação:

$$
\frac{\partial l}{\partial x_{t}}=\sigma_{u u}^{-1} \sum_{i=1}^{r_{t}}\left(X_{t i}-x_{t}\right)+\sigma_{e e}^{-1} \beta \sum_{j=1}^{s_{t}}\left(Y_{t j}-\alpha-\beta x_{t}\right)=0, \quad t=1, \ldots, n .
$$

Agora, ao substituir $x_{t}$ por $g_{t}$ em (4.22) na expressão da log-verossimilhança (4.21) construimos, depois de omitir as constantes desnecessárias, a função:

$$
h[(\mathrm{X}, \mathrm{Y}) ; \theta]=\sum_{t=1}^{n} h_{t}\left[\left(\mathbf{X}_{\mathbf{t}}, \mathbf{Y}_{\mathbf{t}}\right) ; \theta\right]
$$

em que

$$
\begin{aligned}
h_{t}\left[\left(\mathbf{X}_{\mathrm{t}}, \mathbf{Y}_{\mathrm{t}}\right) ; \theta\right]= & -\frac{r_{t}}{2} \ln \sigma_{u u}-\frac{s_{t}}{2} \ln \sigma_{e e}-\frac{1}{2}\left[\sigma_{u u}^{-1} \sum_{i=1}^{r_{t}}\left(X_{t i}-g_{t}\right)^{2}\right. \\
& \left.+\sigma_{e e}^{-1} \sum_{j=1}^{s_{t}}\left(Y_{t j}-\alpha-\beta g_{t}\right)^{2}\right]
\end{aligned}
$$


que, após algumas manipulações algébricas, pode ser escrita como:

$$
\begin{aligned}
h_{t}\left[\left(\mathrm{X}_{\mathrm{t}}, \mathrm{Y}_{\mathrm{t}}\right) ; \theta\right]= & -\frac{r_{t}}{2} \ln \sigma_{u u}-\frac{s_{t}}{2} \ln \sigma_{e e} \\
& -\frac{1}{2}\left\{\sigma_{u u}^{-1} \sum_{i=1}^{r_{t}}\left(X_{t j}-\bar{X}_{t .}\right)^{2}+\sigma_{e e}^{-1} \sum_{j=1}^{s_{t}}\left(Y_{t j}-\bar{Y}_{t .}\right)^{2}\right. \\
& \left.+\frac{r_{t} \sigma_{e e}^{-1} \sigma_{u u}^{-1}}{s_{t}\left(r_{t} \sigma_{u u}^{-1}+s_{t} \beta^{2} \sigma_{e e}^{-1}\right)}\left[\sum_{j=1}^{s_{t}}\left(Y_{t j}-\alpha-\beta \bar{X}_{t .}\right)\right]^{2}\right\} .
\end{aligned}
$$

A função (4.23) desempenhará um papel importante no estudo do comportamento assintótico dos EMVs. A seguir enunciaremos um lema que será útil na demonstração do próximo teorema.

Lema 4.2 Seja $\varphi \in C^{2}$ definida sobre um conjunto aberto e convexo $D \subseteq \mathbf{R}^{m}$, e seja $y^{1} \in \mathbf{D}$ um ponto crítico de $\varphi$, isto é, um ponto onde a derivada de $\varphi$ se anula. Se $Q_{\varphi}\left(y^{1},.\right)<0$, então $y^{1}$ é um ponto de máximo local da função $\varphi$, onde a função $Q_{\varphi}: \mathbf{D} \times \mathbf{R}^{m} \longrightarrow \mathbf{R}$ é definida como

$$
Q_{\varphi}(x, h)=\sum \sum \varphi_{j k}(x) h_{j} h_{k}=\mathbf{h}^{\prime} \Psi \mathrm{h}
$$

e $\Psi$ é uma matriz simétrica de ordem $m$, cujas entradas são os elementos $\varphi_{j k}=\frac{\partial^{2} \varphi(x)}{\partial x_{k} \partial x_{j}}$ com $h \in \mathbf{R}^{m}$. Além disso, para cada $y^{1} \in \mathbf{D}$, a função $Q_{\varphi}$ definida por $Q_{\varphi}^{y^{1}}(h)=$ $Q_{\varphi}\left(y^{1}, h\right)$ é a forma quadrática correspondente à matriz $\left(\varphi_{j k}\left(y^{1}\right)\right)_{m m}$ das segundas derivadas de $\varphi_{j k}\left(y^{1},.\right)$. Assim, $Q_{\varphi}\left(y^{1},.\right)<0$ implica que a matriz $\left(\varphi_{j k}\left(y^{1}\right)\right)_{m m}$ é negativa definida.

Este lema e sua demonstração encontram-se, por exemplo, em Fleming (1966); para mais detalhes ver Vilca-Labra (1996).

Teorema 4.1 Dados os verdadeiros valores dos parâmetros estruturais, $\theta^{\circ}=\left(\alpha^{o}, \beta^{o}\right.$, $\left.\sigma_{e e}^{o}, \sigma_{u u}^{o}\right)^{\prime}$, o EMV de $\theta, \hat{\theta}_{n}$, converge em probabilidade para:

$$
\theta^{1}=\left(\alpha^{o}, \beta^{o},\left(\frac{\bar{r}+\bar{s}-1}{\bar{r}+\bar{s}}\right) \sigma_{e e}^{o},\left(\frac{\bar{r}+\bar{s}-1}{\bar{r}+\bar{s}}\right) \sigma_{u u}^{o}\right)^{\prime},
$$


no qual $\theta^{1} \in \Theta^{\circ}$ é um máximo local da função $\psi(\theta)=n^{-1} \sum_{t=1}^{n} E^{o}\left(h_{t}\left(\mathbf{Z}_{t}, \theta\right)\right), \bar{r}=$ $\frac{\sum_{t=1}^{n} r_{t}}{n}$ e $\bar{s}=\frac{\sum_{t=1}^{n} s_{t}}{n}$, que supomos finitos, para $n$ grande.

Demonstração: De (4.24) temos que:

$$
\begin{aligned}
\mathrm{E}^{o}\left(h_{t}\left[\left(X_{t}, Y_{t}\right)\right]\right) & =-\frac{r_{t}}{2} \ln \sigma_{u u}-\frac{s_{t}}{2} \ln \sigma_{u} u-\frac{1}{2}\left\{\sigma_{u u}^{-1} \mathrm{E}^{o}\left(\sum_{j=1}^{r_{t}}\left(X_{t j}-\bar{X}_{t .}\right)\right)^{2}\right. \\
& +\sigma_{e e}^{-1} \mathrm{E}^{o}\left(\sum_{j=1}^{s_{t}}\left(Y_{t j}-\bar{Y}_{t} .\right)\right)^{2} \\
& \left.+\frac{r_{t} \sigma_{e e}^{-1} \sigma_{u u}^{-1}}{s_{t}\left(r_{t} \sigma_{u u}^{-1}+s_{t} \beta^{2} \sigma_{e e}^{-1}\right)} \mathrm{E}^{o}\left(\left[\sum_{l=1}^{s_{t}}\left(Y_{t l}-\beta \bar{X}_{t} .-\alpha\right)\right]^{2}\right)\right\}
\end{aligned}
$$

Como

$$
\begin{aligned}
& \mathrm{E}^{o}\left(\sum_{j=1}^{r_{t}}\left(X_{t j}-\bar{X}_{t} \cdot\right)^{2}\right)=\left(r_{t}-1\right) \sigma_{u u}^{o} \\
& \mathrm{E}^{o}\left(\sum_{l=1}^{s_{t}}\left(Y_{t l}-\bar{Y}_{t} \cdot\right)^{2}\right)=\left(s_{t}-1\right) \sigma_{e e}^{o}
\end{aligned}
$$

e

$$
\begin{aligned}
\mathrm{E}^{o}\left(\sum_{l=1}^{s_{t}}\left(Y_{t l}-\beta \bar{X}_{t} \cdot-\alpha\right)\right)^{2} & =s_{t}^{2}\left[\left(\alpha-\alpha^{o}\right)+\left(\beta-\beta^{o}\right) x_{t}^{o}\right]^{2} \\
& +s_{t} \sigma_{e e}^{0}+\frac{s_{t}^{2}}{r_{t}} \beta^{2} \sigma_{u u}^{0}
\end{aligned}
$$

temos que

$$
\begin{aligned}
\mathrm{E}^{o}\left(h_{t}\left[\left(\mathrm{X}_{t}, \mathrm{Y}_{t}\right)\right]\right) & =-\frac{r_{t}}{2} \ln \sigma_{u u}-\frac{s_{t}}{2} \ln \sigma_{e e}-\frac{1}{2}\left\{\left(r_{t}-1\right) \sigma_{u u}^{-1} \sigma_{u u}^{0}\right. \\
& +\left(s_{t}-1\right) \sigma_{e e}^{-1} \sigma_{e e}^{o}+\sigma_{e e}^{-1} \sigma_{u u}^{-1} \sigma_{e e}^{0} \sigma_{u u}^{o} \frac{\left(r_{t} \sigma_{u u}^{0}{ }^{-1}+s_{t} \beta^{2} \sigma_{e e}^{0}{ }^{-1}\right)}{r_{t} \sigma_{u u}^{-1}+s_{t} \beta^{2} \sigma_{e e}^{-1}} \\
& \left.+\frac{r_{t} s_{t} \sigma_{e e}^{-1} \sigma_{u u}^{-1}}{r_{t} \sigma_{u u}^{-1}+s_{t} \beta^{2} \sigma_{e e}^{-1}}\left[\left(\alpha-\alpha^{0}\right)+\left(\beta-\beta^{0}\right) x_{t}^{0}\right]^{2}\right\} .
\end{aligned}
$$


Vamos obter, agora, os pontos críticos de (4.29), através das equações das primeiras derivadas parciais da função (4.29), isto é :

$$
\frac{\partial}{\partial \theta_{j}} \frac{\sum_{t=1}^{n} E^{o}\left(h_{t}\right)}{n}=0, \quad \theta=\left(\theta_{1}, \theta_{2}, \theta_{3}, \theta_{4}\right)=\left(\alpha, \beta, \sigma_{u u}, \sigma_{e e}\right) .
$$

Portanto, temos

$$
\frac{\partial}{\partial \alpha}\left[\frac{\sum_{t=1}^{n} E^{o}\left(h_{t}\right)}{n}\right]=\sum_{t=1}^{n} \frac{r_{t} s_{t} \sigma_{e e}^{-1} \sigma_{u u}^{-1}}{r_{t} \sigma_{u u}^{-1}+s_{t} \beta^{2} \sigma_{e e}^{-1}}\left[\left(\alpha-\alpha^{0}\right)+\left(\beta-\beta^{0}\right) x_{t}^{0}\right]=0 ;
$$

$$
\begin{aligned}
& \frac{\partial}{\partial \beta}\left[\frac{\sum_{t=1}^{n} E^{o}\left(h_{t}\right)}{n}\right]=0 \Rightarrow \\
& \quad\left(\sigma_{e e}^{-1} \sigma_{u u}^{-1} \sigma_{e e}^{o} \sigma_{u u}^{o}\right) \sum_{t=1}^{n}\left[\frac{\partial}{\partial \beta}\left(\frac{r_{t}\left(\sigma_{u u}^{o}\right)^{-1}+s_{t} \beta^{2}\left(\sigma_{e e}^{o}\right)^{-1}}{r_{t} \sigma_{u u}^{-1}+s_{t} \beta^{2} \sigma_{e e}^{-1}}\right)\right] \\
& +\sigma_{e e}^{-1} \sigma_{u u}^{-1} \sum_{t=1}^{n}\left\{\left[\left(\alpha-\alpha^{o}\right)+\left(\beta-\beta^{o}\right) x_{t}^{o}\right]^{2}\left[\frac{\partial}{\partial \beta}\left(\frac{r_{t} s_{t}}{r_{t} \sigma_{u u}^{-1}+s_{t} \beta^{2} \sigma_{e e}^{-1}}\right)\right]\right\} \\
& \quad+2 \sum_{t=1}^{n}\left\{\left(\frac{r_{t} s_{t} \sigma_{u u}^{-1} \sigma_{e e}^{-1}}{r_{t} \sigma_{u u}^{-1}+s_{t} \beta^{2} \sigma_{e e}^{-1}}\right)\left[\left(\alpha-\alpha^{o}\right)+\left(\beta-\beta^{o}\right) x_{t}^{o}\right] x_{t}^{o}\right\}=0
\end{aligned}
$$

$$
\begin{aligned}
\frac{\partial}{\partial \sigma_{u u}}\left[\frac{\sum_{t=1}^{n} E^{o}\left(h_{t}\right)}{n}\right] & =0 \quad \Rightarrow \\
& -\bar{r} \sigma_{u u}+(\bar{r}-1) \sigma_{u u}^{o}+\frac{\beta^{2} \sigma_{u u}^{o}}{n} \sum_{t=1}^{n}\left[\frac{s_{t}\left(r_{t} \lambda^{o}+s_{t} \beta^{2}\right)}{\left(r_{t} \lambda+s_{t} \beta^{2}\right)^{2}}\right]=0
\end{aligned}
$$

$$
\begin{aligned}
\frac{\partial}{\partial \sigma_{e e}} & {\left[\frac{\sum_{t=1}^{n} E^{o}\left(h_{t}\right)}{n}\right]=0 \quad \Rightarrow } \\
& -\bar{s} \sigma_{e e}+(\bar{s}-1) \sigma_{e e}^{o} \sigma_{e e}^{-2}+\frac{\sigma_{u u}^{-2} \sigma_{u u}^{o}}{n} \sum_{t=1}^{n}\left\{\frac{r_{t}\left[r_{t}\left(\sigma_{u u}^{o}\right)^{-1} \sigma_{e e}^{o}+s_{t} \beta^{2}\right]}{\left(r_{t} \lambda+s_{t} \beta^{2}\right)^{2}}\right\}=0 .
\end{aligned}
$$


Uma solução possível para (4.30) é:

$$
\alpha^{1}=\alpha^{\circ}, \quad \beta^{1}=\beta^{\circ},
$$

que substituindo em (4.31) resulta em

$$
\sum_{t=1}^{n}\left(\frac{1}{r_{t} \lambda+s_{t} \beta^{2}}\right)-\sum_{t=1}^{n}\left[\frac{r_{t} \lambda^{\circ}+s_{t} \beta^{2}}{\left(r_{t} \lambda+s_{t} \beta^{2}\right)^{2}}\right]=0,
$$

em que $\lambda=\frac{\sigma_{e e}}{\sigma_{u u}}$ e $\lambda^{o}=\frac{\sigma_{e e}^{o}}{\sigma_{u u}^{o}}$.

Uma solução possível para (4.31) é:

$$
\lambda=\lambda^{o}, \quad \text { isto é, } \quad \frac{\sigma_{e e}}{\sigma_{u u}}=\frac{\sigma_{e e}^{o}}{\sigma_{u u}^{o}} .
$$

Note este resultado é uma suposição adotada em Mak (1982). Podemos interpretá-lo da seguinte maneira: a razão entre as variâncias dos erros de mensuração é a mesma, tanto no ponto crítico como entre os verdadeiros valores desses parâmetros. Substituindo (4.36) em (4.32) e (4.33) temos, respectivamente,

$$
-\bar{r} \sigma_{u u}+\bar{r} \sigma_{u u}^{o}-\frac{\lambda \sigma_{u u}^{o}}{n} \sum_{t=1}^{n}\left(\frac{r_{t}}{r_{t} \lambda+s_{t} \beta^{2}}\right)=0
$$

$\mathrm{e}$

$$
\frac{\lambda \sigma_{u u}^{o}}{n} \sum_{t=1}^{n}\left(\frac{r_{t}}{r_{t} \lambda+s_{t} \beta^{2}}\right)=\bar{s} \sigma_{u u}+(\bar{s}-1) \sigma_{u u}^{o} .
$$

De (4.37), (4.38) e (4.36) temos que

$$
\sigma_{u u}^{(1)}=\left(\frac{\bar{r}+\bar{s}-1}{\bar{r}+\bar{s}}\right) \sigma_{u u}^{o} \quad \text { e } \quad \sigma_{e e}^{(1)}=\left(\frac{\bar{r}+\bar{s}-1}{\bar{r}+\bar{s}}\right) \sigma_{e e}^{o} .
$$

Portanto, $\theta^{1}=\left(\alpha^{0}, \beta^{0}, \frac{\bar{r}+\bar{s}-1}{\bar{r}+\bar{s}} \sigma_{u u}^{0}, \frac{\bar{r}+\bar{s}-1}{\bar{r}+\bar{s}} \sigma_{e e}^{0}\right)$ é uma raíz das equações de derivadas parciais de $\frac{\partial}{\partial \theta_{j}} \sum_{t=1}^{n} E\left(h_{t}\right) / n=0, j=1, \ldots, 4$, a única raíz possível dentro do espaço paramétrico (independente dos valores de $x_{t}^{0}$ ), ou seja, $\theta^{1}$ é um ponto crítico da função $\sum_{t=1}^{n} E\left(h_{t}\right) / n$.

Agora, façamos: $\psi(\theta)=n^{-1} \sum_{t=1}^{n} E^{o}\left(h_{t}\left(\mathrm{Z}_{t}, \theta\right)\right)$, como no Lema 3.1. Portanto, $\theta^{1}$ 
também é um ponto crítico de $\psi(\theta)$, isto é, $\left.\frac{\partial}{\partial \theta_{j}}(\psi(\theta))\right|_{\theta=\theta^{(1)}}=0$.

Por outro lado, temos que a matriz

$$
\begin{aligned}
\left(\psi_{j k}\left(\theta^{(1)}\right)\right)_{44} & =\left.\left(\frac{\partial^{2}}{\partial \theta_{k} \theta_{j}} \psi(\theta)\right)\right|_{\theta=\theta^{(1)}}=\left.\left\{\frac{\partial^{2}}{\partial \theta_{k} \theta_{j}}\left(n^{-1} \sum_{t=1}^{n} E^{o}\left[h_{t}\left(\mathbf{Z}_{t}, \theta\right)\right]\right)\right\}\right|_{\theta=\theta^{(1)}} \\
& =\left.\left(n^{-1} \sum_{t=1}^{n} E^{o}\left[\frac{\partial^{2}}{\partial \theta_{k} \theta_{j}} h_{t}\left(\mathbf{Z}_{t}, \theta\right)\right]\right)\right|_{\theta=\theta^{(1)}},
\end{aligned}
$$

onde a última igualdade resulta das condições de regularidade adotadas e do teorema de convergência dominada. Pela notação adotada temos que:

$$
\left(\psi_{j k}\left(\theta^{(1)}\right)\right)_{44}=E^{o}\left[\mathbf{A}_{n}\left(\theta^{(1)}\right)\right] .
$$

A prova de que $E^{o}\left[\mathbf{A}_{n}\left(\theta^{(1)}\right)\right]$ (esta matriz será calculada mais adiante) seja negativa definida envolve algumas suposições adicionais sobre a seqüência $\left\{x_{t}\right\}_{t \geq 1}$ e está no Apêndice D. Aceitando-se estas suposições temos pelo Lema 4.2 que $\theta^{1} \in \Theta^{\circ}$ é um máximo local da função $\psi(\theta)=n^{-1} \sum_{t=1}^{n} E^{o}\left(h_{t}\left(\mathrm{Z}_{t}, \theta\right)\right)$.

A demonstração da primeira parte do teorema está dada em Mak (1982).

Portanto, a observação (1) da Seção 2 em Mak (1982) (ver Apêndice B.3) se mantém com

$$
\theta^{1}=\left(\alpha^{0}, \beta^{0}, \frac{\bar{r}+\bar{s}-1}{\bar{r}+\bar{s}} \sigma_{u u}^{0}, \frac{\bar{r}+\bar{s}-1}{\bar{r}+\bar{s}} \sigma_{e e}^{0}\right),
$$

ou seja, o segundo resultado do teorema anterior substitui as condições (C-1) e (C-2) de regularidade. Além disso, as condições $(C-3)$ a $(C-6)$ e $(C-8)$ são satisfeitas desde que:

$$
0<\lim _{n \rightarrow \infty} \inf \sum_{t=1}^{n} \frac{\left(x_{t}^{(o)}-\bar{x}^{(o)}\right)^{2}}{n} \leq \lim _{n \rightarrow \infty} \sup \frac{1}{n} \sum_{t=1}^{n}\left(x_{t}^{(o)}-\bar{x}^{(o)}\right)^{2}<\infty,
$$

pois isto implica que

$$
0<\lim _{n \rightarrow \infty} \inf V_{n} \leq \lim _{n \rightarrow \infty} \sup V_{n}<\infty \quad \text { e } \quad\left\{E^{0}\left[A_{n} \theta^{(1)}\right]\right\}^{-1}<\infty
$$


As equações de verossimilhança que possibilitam a obtenção do $\operatorname{EMV} \theta, \hat{\theta}_{n}$, serão deduzidas mais adiante, e esta solução é a única admissivel (Teorema 2.1-b de Mak(1982)). Se além disso existir um $\gamma>0$ tal que

$$
\lim _{n \rightarrow \infty} \frac{1}{n^{1+\frac{\gamma}{2}}} \sum_{t=1}^{n}\left|x_{t}\right|^{2+\gamma}=0,
$$

então a condição $(\mathrm{C}-7)$ é satisfeita, e pelo teorema (2.3) de Mak (1982), $\hat{\theta_{n}}$ é assintoticamente normal com média $\left(\theta^{0}, \beta^{0}, \frac{\bar{r}+\bar{s}-1}{\bar{r}+\bar{s}} \sigma_{u u}^{0}, \frac{\bar{r}+\bar{s}-1}{\bar{r}+\bar{s}} \sigma_{e e}^{0}\right)$ e matriz de covariância dada por

$$
n^{-1}\left\{E^{o}\left[\mathbf{A}_{n}\left(\theta^{(1)}\right)\right]\right\}^{-1} \mathrm{~V}_{n}\left\{E^{o}\left[\mathbf{A}_{n}\left(\theta^{(1)}\right)\right]\right\}^{-1} .
$$

Notemos que o EMV de $\theta$ é consistente para $\alpha$ e $\beta$ mas não para $\sigma_{u u}$ e $\sigma_{e e}$. Como apontado anteriormente, a função $T\left(\hat{\theta}_{n}\right)=\left(\hat{\alpha}, \hat{\beta}, \frac{\bar{r}+\bar{s}}{\bar{r}+\bar{s}-1} \hat{\sigma}_{u u}, \frac{\bar{r}+\bar{s}}{\bar{r}+\bar{s}-1} \hat{\sigma}_{e e}\right)$ do EMV $\hat{\theta_{n}}=\left(\hat{\alpha}, \hat{\beta}, \hat{\sigma}_{u u}, \hat{\sigma}_{e e}\right)$ é consistente para $\theta$. A inconsistência de $\hat{\sigma}_{u u}$ no caso sem replicação mas com a suposição da razão $\lambda^{\circ}=\sigma_{e e}^{o} / \sigma_{u u}^{o}$ conhecida, foi observada na literatura por Lindley (1945), e a consistência de $\hat{\alpha}$ e $\hat{\beta}$ foi demostrada por Lindley (1945), Kendall e Stuart (1973), entre outros, usando um método que requer as convergências de $\bar{x}_{n}^{(o)}$ e $S_{x^{(o)} x^{(o)}}$ para limites finitos. Aqui, requeremos somente que $\bar{x}^{(0)}$ não sejam demasiadamente dispersos e nem concentrados, quando $n \rightarrow \infty$ (ver a condição dada em (4.40)), ou seja, não se supõe que $\lim _{n \rightarrow \infty} \bar{x}^{0}$ e $\lim _{n \rightarrow \infty} \frac{\sum_{t=1}^{n}\left(x_{t}^{(o)}\right)^{2}}{n}$ existam.

Alguns casos especiais do resultado acima (Teorema 3.1) são estabelecidos no corolário a seguir.

Corolário 4.1 Sob a suposição do Teorema 3.1, temos que

(a) Se $\frac{s_{t}}{r_{t}}=k, t=1, \ldots, n$, então o EMV de $\theta, \hat{\theta}_{n}$, converge para

$$
\theta^{(1)}=\left\{\alpha^{0}, \beta^{0},\left(\frac{r+k-1 / \bar{r}}{1+k}\right) \sigma_{u u}^{(o)},\left(\frac{r+k-1 / \bar{r}}{1+k}\right) \sigma_{e e}^{(o)}\right\} .
$$

(b) Se $r_{t}=r$, e $s_{t}=s, t=1, \cdots, n$ então o EMV de $\theta$ converge para

$$
\theta^{(1)}=\left\{\alpha^{0}, \beta^{0},\left(\frac{r+s-1}{r+s}\right) \sigma_{u u}^{(o)},\left(\frac{r+s-1}{r+s}\right) \sigma_{e e}^{(o)}\right\} .
$$


(c) $S e r_{t}=r$ e $s_{t}=1, t=1, \ldots, n$, então o EMV de $\theta$ converge para

$$
\theta^{1}=\left(\alpha^{0}, \beta^{0}, \frac{r}{r+1} \sigma_{u u}^{0}, \frac{r}{r+1} \sigma_{e e}^{0}\right)
$$

O item (c) no Corolário 4.1 é um caso muito considerado na prática. Esquemas de replicação mais gerais como aqueles apresentados em Fuller (1987), por exemplo, também podem ser considerados.

A seguir examinamos a distribuição assintótica do EMV, $\hat{\theta_{n}}$, e, então, deduzimos a distribuição assintótica do EMV consistente de $\theta^{(o)}$,

$$
\tilde{\theta}_{n}=T\left(\hat{\theta_{n}}\right)=\left(\hat{\alpha}, \hat{\beta},\left[\frac{\bar{r}+\bar{s}-1}{\bar{r}+\bar{s}}\right]^{-1} \hat{\sigma_{u u}},\left[\frac{\bar{r}+\bar{s}-1}{\bar{r}+\bar{s}}\right]^{-1} \hat{\sigma_{e e}}\right) .
$$

Pelo Teorema 2.3 em Mak (1982) $\tilde{\theta}_{n}$ é um estimador consistente de $\theta^{(o)}$ e é assintóticamente normal com média $T\left(\theta^{(1)}\right)=\theta^{(0)}$ e matriz de covariância

$$
n^{-1} D\left\{E^{0}\left[A_{n}\left(\theta^{1}\right)\right]\right\}^{-1} V_{n}\left\{E^{0}\left[A_{n}\left(\theta^{1}\right)\right]\right\}^{-1} D^{\prime},
$$

no qual D é a matriz cujo elemento $(i, j)$ é $\left[\frac{\partial T_{i}(\theta)}{\partial \theta_{j}}\right]$, e $T_{i}(\theta)$ é a i-ésima componente da função $T(\theta)=\left(\alpha, \beta,\left[\frac{\bar{r}+\bar{s}-1}{\bar{r}+\bar{s}}\right]^{-1} \sigma_{u u},\left[\frac{\bar{r}+\bar{s}-1}{\bar{r}+\bar{s}}\right]^{-1} \sigma_{e e}\right)$. Portanto, temos que

$$
\mathbf{D}=\left(\frac{\partial T_{i}(\theta)}{\partial \theta_{j}}\right)=\operatorname{diagonal}\{1, \quad 1, \quad \bar{w}, \quad \bar{w}\}, \quad \text { com } \quad \bar{w}=\frac{\bar{r}+\bar{s}}{\bar{r}+\bar{s}-1} .
$$

\subsection{Cálculos dos EMVs de $\left(\alpha^{(o)}, \beta^{(o)}, \sigma_{u u}^{(o)}, \sigma_{e e}^{(o)}\right)^{\prime}$}

Usando a mesma notação de Mak (1982), as seguintes expressões são obtidas ao deduzir a função $h_{t}\left[\left(\mathrm{X}_{t}, \mathrm{Y}_{t}\right) \mid \theta\right]=h_{t}$ dada em (4.24), que para facilidade de notação, denotaremos por $h_{t}$, em relação a $\alpha, \beta, \sigma_{u u} e \sigma_{e e}$ :

$$
q_{t \alpha}=\frac{\partial h_{t}}{\partial \alpha}=\left(\frac{r_{t} \sigma_{e e}^{-1} \sigma_{u u}^{-1}}{r_{t} \sigma_{u u}^{-1}+s_{t} \beta^{2} \sigma_{e e}^{-1}}\right) \sum_{l=1}^{s_{t}}\left(y_{t l}-\beta \bar{X}_{t .}-\alpha\right)
$$




$$
\begin{gathered}
q_{t \beta}=\frac{\partial h_{t}}{\partial \beta}=\frac{r_{t} \sigma_{u u}^{-1} \sigma_{e e}^{-2}}{\left(r_{t} \sigma_{u u}^{-1}+s_{t} \beta^{2} \sigma_{e e}^{-1}\right)^{2}}\left[\sum_{l=1}^{s_{t}}\left(Y_{t l}-\beta \bar{X}_{t .}-\alpha\right)\right]\left[\sum_{l=1}^{s_{t}}\left(\beta Y_{t l}+\frac{r_{t}}{s_{t}} \lambda \bar{X}_{t .}-\beta \alpha\right)\right] \\
q_{t \sigma_{u u}}=\frac{\partial h_{t}}{\partial \sigma_{u u}}=-\frac{1}{2} r_{t} \sigma_{u u}^{-1}+\frac{1}{2} \sigma_{u u}^{-2} \sum_{j=1}^{r_{t}}\left(X_{t j}-\bar{X}_{t .}\right)^{2} \\
+\frac{1}{2}\left(\frac{r_{t} \beta^{2} \sigma_{e e}^{-2} \sigma_{u u}^{-2}}{\left(r_{t} \sigma_{u u}^{-1}+s_{t} \beta^{2} \sigma_{e e}^{-1}\right)^{2}}\right)\left[\sum_{l=1}^{s_{t}}\left(Y_{t l}-\beta \bar{X}_{t .}-\alpha\right)\right]^{2} \\
q_{t \sigma_{e e}=-} \\
+\frac{1}{2} s_{t} \sigma_{e e}^{-1}+\frac{1}{2} \sigma_{e e}^{-2} \sum_{l=1}^{s_{t}}\left(\frac{r_{t}^{2} \sigma_{u u}^{-2} \sigma_{e e}^{-2}}{s_{t}\left(r_{t} \sigma_{u u}^{-1}+s_{t} \beta^{2} \sigma_{e e}^{-1}\right)^{2}}\right)\left[\sum_{l=1}^{s_{t}}\left(Y_{t l}-\beta \bar{X}_{t .}-\alpha\right)\right]^{2}
\end{gathered}
$$

Sem nenhuma suposição sobre $r_{t}$ e $s_{t}$, as equações de máxima verossimilhança não têm uma solução analítica, pelos mesmos argumentos apresentados no caso do modelo estrutural. Neste caso, usamos um processo iterativo proposto por Kimura (1992), que é um algoritmo tipo EM, para obter os EMVs.

Portanto, a suposição mais geral, que favorece uma solução analítica é considerar a razão entre os números de replicações constante, isto é, $s_{t} / r_{t}=k$. Assim, de (4.45) temos

$$
\sum_{t=1}^{n} q_{t \alpha}=0 \Rightarrow \sum_{t=1}^{n} \sum_{l=1}^{s_{t}}\left(Y_{t l}-\beta \bar{X}_{t .}-\alpha\right)=0 \Rightarrow \bar{Y}_{. .}-\hat{\alpha}-\hat{\beta} \bar{X}_{. .}=0,
$$

em que $\quad \bar{Y}_{. .}=\frac{\sum_{t=1}^{n} \sum_{l=1}^{s_{t}} Y_{t l}}{\sum_{t=1}^{n} s_{t}}, \quad \bar{X}_{. .}=\frac{\sum_{t=1}^{n} \sum_{j=1}^{r_{t}} X_{t j}}{\sum_{t=1}^{n} r_{t}} \quad$ e $\quad \frac{\sum s_{t}}{\sum r_{t}}=k$

De (4.46), $\lambda=\sigma_{e e} / \sigma_{u u}$ e (4.49), temos

$$
\begin{gathered}
\sum_{t=1}^{n} q_{t \beta}=0 \Rightarrow k \sum_{t=1}^{n}\left[\left(\beta \bar{Y}_{t .}+\frac{\lambda}{k} \bar{X}_{t .}-\beta \alpha\right) \sum_{l=1}^{s_{t}}\left(Y_{t l}-\beta \bar{X}_{t .}-\alpha\right)\right]=0 \Rightarrow \\
\hat{\lambda}=\frac{k \hat{\beta}\left(\hat{\beta} S_{X Y}^{*}-S_{Y Y}^{*}\right)}{S_{X Y}^{*}-\beta S_{X X}^{*}}
\end{gathered}
$$


na qual $\quad S_{X X}^{*}=\sum_{t=1}^{n} \frac{r_{t}\left(\bar{X}_{t .}-\bar{X}_{. .}\right)^{2}}{\sum_{t=1}^{n} r_{t}}, \quad S_{Y Y}^{*}=\sum_{t=1}^{n} \frac{s_{t}\left(\bar{Y}_{t .}-\bar{X}_{0 .}\right)^{2}}{\sum_{t=1}^{n} s_{t}}$

e

$$
S_{X Y}^{*}=\sum_{t=1}^{n} \frac{s_{t}\left(\bar{X}_{t .}-\bar{X}_{. .}\right)\left(\bar{Y}_{t .}-\bar{Y}_{. .}\right)}{\sum_{t=1}^{n} s_{t}}
$$

De (4.47) temos

$$
\begin{gathered}
\sum_{t=1}^{n} q_{t} \sigma_{u u}=0 \quad \Longrightarrow \quad-\frac{\sigma_{u u}^{-1}}{2} \sum_{t=1}^{n} r_{t}+\frac{1}{2} \sigma_{u u}^{-2} \sum_{t=1}^{n} \sum_{j=1}^{r_{t}}\left(\bar{X}_{t j}-\bar{X}_{t .}\right)^{2} \\
+\left[\frac{\hat{\beta}^{2} \hat{\sigma}_{e e}^{-2} \hat{\sigma}_{u u}^{-2}}{2\left(\hat{\sigma}_{u u}^{-1}+k \hat{\beta}^{2} \hat{\sigma}_{e e}^{-1}\right)^{2}}\right] \sum_{t=1}^{n}\left\{\frac{1}{r_{t}}\left[\sum_{l=1}^{s_{t}}\left(Y_{t l}-\beta \bar{x}_{t .}-\alpha\right)\right]^{2}\right\}=0 .
\end{gathered}
$$

Mas

$$
\sum_{t=1}^{n}\left\{\frac{1}{r_{t}}\left[\sum_{l=1}^{s_{t}}\left(Y_{t l}-\beta \bar{X}_{t .}-\alpha\right)\right]^{2}\right\}=k \sum_{t=1}^{n} s_{t}\left\{S_{Y Y}^{*}+\hat{\beta}^{2} S_{X X}^{*}-2 \hat{\beta} S_{X Y}^{*}\right\} .
$$

Substituindo (4.52) na equação (4.51), temos

$$
\hat{\sigma}_{u u}=W_{X X}^{*}+\frac{k^{2} \beta^{2}}{\left(\hat{\lambda}+k \beta^{2}\right)^{2}}\left(S_{Y Y}^{*}+\beta^{2} S_{X X}^{*}-2 \hat{\beta} S_{X Y}^{*}\right) .
$$

De (4.48) temos

$$
\begin{gathered}
\sum_{t=1}^{n} q_{t} \sigma_{e e}=0 \Longrightarrow-\frac{\sigma_{e e}^{-1}}{2} \sum_{t=1}^{n} s_{t}+\frac{1}{2} \sigma_{e e}^{-1} \sum_{t=1}^{n} \sum_{l=1}^{s_{t}}\left(Y_{t l}-\bar{Y}_{t .}\right)^{2} \\
+\frac{1}{2} \frac{\sigma_{u u}^{-2} \sigma_{e e}^{-2}}{\left(\sigma_{u u}^{-1}+k \beta^{2} \sigma_{e e}^{-1}\right)^{2}} \sum_{t=1}^{n}\left\{\frac{1}{s_{t}}\left[\sum_{l=1}^{s_{t}}\left(Y_{t l}-\beta \bar{x}_{t .}-\alpha\right)\right]^{2}\right\}=0 .
\end{gathered}
$$

Mas

$$
\sum_{t=1}^{n}\left\{\frac{1}{s_{t}}\left[\sum_{l=1}^{s_{t}}\left(Y_{t l}-\beta \bar{x}_{t .}-\alpha\right)\right]^{2}\right\}=\left(\sum_{t=1}^{n} s_{t}\right)\left(S_{Y Y}^{*}+\beta^{2} S_{X X}^{*}-2 \hat{\beta} S_{X Y}\right)
$$

e substituindo (4.55) na equação (4.54), temos 


$$
\hat{\sigma}_{e e}=W_{Y Y}^{*}+\frac{\lambda^{2}}{\left(\lambda+k \beta^{2}\right)^{2}}\left(S_{Y Y}^{*}+\beta^{2} S_{X X}^{*}-2 \beta S_{X Y}^{*}\right)
$$

com

$$
W_{Y Y}^{*}=\frac{\sum_{t=1}^{n} \sum_{l=1}^{s_{t}}\left(Y_{t l}-\bar{Y}_{t .}\right)^{2}}{\sum_{t=1}^{n} s_{t}} .
$$

De (4.53) e (4.56) obtemos

$$
\lambda W_{X X}^{*}-W_{Y Y}^{*}+\frac{\lambda\left(k^{2} \beta^{2}-\lambda\right)}{\left(\lambda+k \beta^{2}\right)^{2}}\left(S_{Y Y}^{*}+\beta^{2} S_{X X}^{*}-2 \hat{\beta} S_{X Y}^{*}\right)=0 .
$$

Como

$$
\frac{k^{2} \beta^{2}-\lambda}{\left(\lambda+k \beta^{2}\right)^{2}}=\left(\frac{k \beta S_{X Y}^{*}-k \beta^{2} S_{X X}^{*}-\beta S_{X Y}^{*}+S_{Y Y}^{*}}{k \beta\left(S_{Y Y}^{*}+\beta^{2} S_{X X}^{*}-2 \beta S_{X Y}^{*}\right)^{2}}\right)\left(S_{X Y}^{*}-\beta S_{X X}^{*}\right),
$$

substituindo (4.57) em (4.58), chegamos a

$$
\begin{aligned}
& k \hat{\beta}\left(\beta S_{X Y}^{*}-S_{Y Y}^{*}\right) W_{X X}^{*}\left(S_{Y Y}^{*}+\beta^{2} S_{X X}^{*}-2 \beta S_{X Y}^{*}\right) \\
& -W_{Y Y}^{*}\left(S_{X Y}^{*}-\beta S_{X X}^{*}\right)\left(S_{Y Y}^{*}-2 \beta S_{X Y}^{*}+\beta^{2} S_{X X}^{*}\right) \\
& +\left(\beta S_{X Y}^{*}-S_{Y Y}^{*}\right)\left(S_{X Y}^{*}-\hat{\beta} S_{X X}^{*}\right)\left(k \beta S_{X Y}^{*}-k \beta^{2} S_{X X}^{*}-\beta S_{X Y}^{*}+S_{Y Y}^{*}\right)=0 .
\end{aligned}
$$

Esta equação em $\beta$ equivale a

$$
a_{4} \beta^{4}+a_{3} \beta^{3}+a_{2} \beta^{2}+a_{1} \beta+a_{0}=0
$$

em que

$$
\begin{aligned}
a_{4}= & k S_{X X}^{*} S_{X Y}^{*} W_{X X}^{*}+k S_{X X}^{* 2} S_{X Y}^{*} \\
a_{3}= & -2 k S_{X Y}^{* 2} W_{X X}^{*}-k S_{X X}^{*} S_{Y Y}^{* 2} W_{X X}^{*}+S_{X X}^{* 2} W_{Y Y}^{*}-(2 k-1) S_{X X}^{*} S_{X Y}^{* 2} \\
& -k S_{X X}^{* 2} S_{Y Y}^{*}
\end{aligned}
$$




$$
\begin{aligned}
& a_{2}=3 k S_{X Y}^{*} S_{Y Y}^{*} W_{X X}^{*}-3 S_{X X}^{*} S_{X Y}^{*} W_{Y Y}^{*}+(k-1) S_{X Y}^{* 3}+(2 k-2) S_{X X}^{*} S_{X Y}^{*} S_{Y Y}^{*} \\
& a_{1}=-k S_{Y Y}^{* 2} W_{X X}^{*}+2 S_{X Y}^{* 2} W_{Y Y}^{* 2}+S_{X X}^{*} S_{Y Y}^{*} W_{Y Y}^{*}+(2-k) S_{X Y}^{* 2} S_{Y Y}^{*}+S_{X X}^{*} S_{Y Y}^{* 2} \\
& a_{0}=-S_{X Y}^{*} S_{Y Y}^{*} W_{Y Y}^{*}-S_{X Y}^{*} S_{Y Y}^{* 2} .
\end{aligned}
$$

Usando as seguintes identidades

$$
t_{X X}^{*}=W_{X X}^{*}+S_{X X}^{*}, t_{Y Y}^{*}=W_{Y Y}^{*}+S_{Y Y}^{*}
$$

podemos simplificar os coeficientes da equação (4.61), tornando-os:

$$
\begin{aligned}
a_{4}= & k S_{X X}^{*} S_{X Y}^{*} t_{X X}^{*} \\
a_{3}= & -2 k S_{X Y}^{* 2} t_{X X}^{*}-k S_{X X}^{*} S_{Y Y}^{*} t_{X X}^{*}+S_{X X}^{* 2} W_{Y Y}^{*}+S_{X X}^{*} S_{X Y}^{* 2}, \\
a_{2}= & 2 k S_{X Y}^{*} S_{Y Y}^{*} t_{X X}^{*}+k S_{X Y}^{*} S_{Y Y}^{*} W_{X X}^{*}-S_{X X}^{*} S_{X Y}^{*} W_{Y Y}^{*}-2 S_{X X}^{*} S_{X Y}^{*} t_{Y Y}^{*} \\
& +(k-1) S_{X Y}^{* 3} \\
a_{1}= & 2 S_{X Y}^{* 2} t_{Y Y}^{*}-k S_{Y Y}^{* 2} W_{X X}^{*}+S_{X X}^{*} S_{Y Y}^{*} t_{Y Y}^{*}-k S_{X Y}^{* 2} S_{Y Y}^{*} \\
a_{0}= & -S_{X Y}^{*} S_{Y Y}^{*} t_{Y Y}^{*}
\end{aligned}
$$

em que

$$
t_{X X}^{*}=\frac{\sum_{t=1}^{n} \sum_{j=1}^{r_{t}}\left(X_{t j}-\bar{X}_{. .}\right)^{2}}{\sum_{t=1}^{n} r_{t}}
$$

$\mathrm{e}$

$$
\begin{gathered}
S_{X X}^{*}=\frac{\sum_{t=1}^{n}\left(\frac{\left[\sum_{j=1}^{r_{t}}\left(X_{t j}-\bar{X}_{. .}\right]^{2}\right.}{r_{t}}\right)}{\sum_{t=1}^{n} r_{t}}=\frac{\sum_{t=1}^{n}\left(X_{t j}-\bar{X}_{. .}\right)^{2}}{\sum_{t=1}^{n} r_{t}} \\
t_{X X}^{*}=W_{X X}^{*}+S_{X X}^{*} .
\end{gathered}
$$

\subsubsection{Casos Particulares}

Caso 1: $r_{t}=r$ e $s_{t}=s$. Temos 
(1) $\quad k=\frac{s}{r}, \quad$ (2) $t_{X X}^{*}=t_{X X}=\frac{\sum_{t=1}^{n} \sum_{j=1}^{r}\left(X_{t j}-\bar{X}_{. .}\right)^{2}}{n . r}$

(3) $S_{X X}^{*}=S_{X X}=\frac{\sum_{t=1}^{n}\left(X_{t .}-\bar{X}_{. .}\right)^{2}}{n}, \quad$ (4) $W_{X X}^{*}=W_{X X}=\frac{\sum_{t=1}^{n} \sum_{j=1}^{r}\left(X_{t j}-\bar{X}_{t .}\right)^{2}}{n . r}$,

(5) $S_{X Y}^{*}=S_{X Y}=\frac{\sum_{t=1}^{n}\left(X_{t .}-\bar{X}_{. .}\right)\left(\bar{Y}_{t .}-\bar{Y}_{. .}\right)}{n}, \quad$ (6) $W_{Y Y}^{*}=W_{Y Y}=\frac{\sum_{t=1}^{n} \sum_{l=1}^{s}\left(Y_{t l}-\bar{Y}_{t .}\right)^{2}}{n . s}$,

(7) $\quad t_{Y Y}^{*}=\frac{\sum_{t=1}^{n} \sum_{j=1}^{s}\left(Y_{t l}-\bar{Y}_{t .}\right)^{2}}{n . s}$.

Os coeficientes da equação de $4^{\circ}$ grau em $\beta$ são similares aos de (4.62) com as modificações nas estatísticas dadas acima.

Caso 2: $r_{t}=s_{t}=r$. Temos

(1) $\quad k=1, \quad(2) t_{X X}^{*}=t_{X X}=\frac{\sum_{t=1}^{n} \sum_{j=1}^{r}\left(X_{t j}-\bar{X}_{. .}\right)^{2}}{n . r}$,

(3) $S_{X X}^{*}=S_{X X},(4) W_{X X}^{*}=W_{X X}, \quad$ (5), $t_{Y Y}^{*}=t_{Y Y}=\frac{\sum_{t=1}^{n} \sum_{l=1}^{r}\left(Y_{t l}-\bar{Y}_{. .}\right)^{2}}{n . r}$

(6) $\quad W_{Y Y}^{*}=W_{Y Y}=\frac{\sum_{t=1}^{n} \sum_{l=1}^{r}\left(Y_{t l}-\bar{Y}_{t .}\right)^{2}}{n . r}, \quad$ (7) $t_{Y Y}^{*}=t_{Y Y}=\frac{\sum_{t=1}^{n} \sum_{l=1}^{r}\left(Y_{t l}-\bar{Y}_{. .}\right)^{2}}{n . r}$.

Substituindo as igualdades acima em (4.62) obtemos os coeficientes da equação de $4^{\circ}$ grau em $\beta$.

Caso 3: $r_{t}=r$ e $s_{t}=1$. Temos,

$$
\begin{gathered}
k=\frac{1}{r}, \quad S_{Y Y}^{*}=t_{Y Y}^{*}=S_{Y Y}=\frac{\sum_{t=1}^{n}\left(Y_{t}-\bar{Y}\right)^{2}}{n} \\
W_{Y Y}^{*}=0, \quad S_{X X}^{*}=S_{X X}, \quad t_{X X}^{*}=W_{X X}
\end{gathered}
$$

tal que a equação de $4^{\circ}$ grau pode ser escrita como:

$$
\begin{aligned}
& \left(\hat{\beta} S_{X Y}-S_{Y Y}\right)\left\{\beta^{3} S_{X X} t_{X X}+\beta^{2}\left(r S_{X Y} S_{X X}-2 S_{X Y} t_{X X}\right)\right. \\
& \left.+\beta\left[S_{Y Y} t_{Y Y}-(r-1) S_{X Y}^{2}-(r-1) S_{Y Y} S_{X X}\right]+S_{X Y} S_{Y Y}\right\}=0 .
\end{aligned}
$$


Portanto, a equação de quarto grau se reduz a uma de terceiro, pois a raíz $\hat{\beta}=\frac{S_{Y Y}}{S_{X Y}} \Rightarrow$ $\hat{\lambda}=0$, ou seja, fica fora do espaço paramétrico.

\subsection{Matriz de Covariância Assintótica dos EMVs de $\theta^{0}$}

Obtemos a seguir a matriz de covariâncias assintóticas considerando o caso geral em relação ao número de replicações, isto é, fazendo $r_{t}$ e $s_{t}$ quaisquer, para $t=1, \ldots, n$.

Como visto anteriormente esta matriz pode ser obtida através da seguinte fórmula (Mak, 1982)

$$
n^{-1}\left\{E\left[A_{n}\left(\theta^{1}\right)\right]\right\}^{-1} V_{n}\left\{E\left[\left(A_{n}\left(\theta^{1}\right)\right]\right\}^{-1},\right.
$$

em que $A_{n}(\theta)$, como definida anteriormente, é a matriz cujo elemento (k,l) é dado por $a_{k l}=\frac{\sum_{t=1}^{n} q_{t k l}}{n}, \operatorname{com} q_{t k l}=\frac{\partial^{2} h_{t}\left\{\left[\left(X_{t}, Y_{t}\right), \theta\right]\right\}}{\partial \theta_{l} \partial \theta_{k}}$. Aqui, $q_{t k l}$ é uma notação simplificada de $q_{t \theta_{k} \theta_{l}}\left[z_{t}, \theta\right]$, dada em (4.9), e

$$
V_{n}=\sum_{t=1}^{n} \frac{\operatorname{cov}\left(q_{t}\left[\left(X_{t}, Y_{t}\right), \theta^{1}\right] / \theta^{0}, x_{t}^{0}\right)}{n}=\frac{\sum_{t=1}^{n} \operatorname{cov}^{0}\left[q_{t}\left(\theta^{1}\right)\right]}{n}
$$

com

$$
q_{t}\left(\theta^{1}\right)=\left\{q_{t \alpha}\left(\theta^{1}\right), q_{t \beta}\left(\theta^{1}\right), q_{t \sigma_{u u}}\left(\theta^{1}\right), q_{t \sigma_{e e}}\left(\theta^{1}\right)\right\}
$$

\subsubsection{Cálculo da Matriz $E\left[A_{n}\left(\theta^{1}\right)\right]$}

As primeiras derivadas parciais da função $h_{t}, q_{t \theta_{j}}, \theta_{j}=\alpha, \beta, \sigma_{u u}, \sigma_{e e}$, para o cálculo de $E\left[A n\left(\theta^{1}\right)\right]$, são dadas na Seção 3.4. As segundas derivadas da função $h_{t}$ são, agora, consideradas.

(i) Cálculos de $q_{t \alpha l}, l=\alpha, \beta, \sigma_{u u}, \sigma_{e e}$

. De (4.45) temos 


$$
\begin{aligned}
q_{t \alpha \alpha}= & \frac{\partial q_{t \alpha}}{\partial \alpha}=-\frac{r_{t} s_{t} \sigma_{e e}^{-1} \sigma_{u u}^{-1}}{r_{t} \sigma_{u u}^{-1}+s_{t} \beta^{2} \sigma_{e e}^{-1}} \\
q_{t \alpha \beta}= & \frac{\partial q_{t \alpha}}{\partial \beta}=r_{t} \sigma_{e e}^{-1} \sigma_{u u}^{-1}\left\{-\frac{2 \beta s_{t} \sigma_{e e}^{-1}}{\left(r_{t} \sigma_{u u}^{-1}+s_{t} \beta^{2} \sigma_{e e}^{-1}\right)^{2}} \times \sum_{l=1}^{s_{t}}\left(Y_{t l}-\beta \bar{X}_{t .}-\alpha\right)\right. \\
& \left.-\frac{s_{t} \bar{X}_{t .}}{\left(r_{t} \sigma_{u u}^{-1} s_{t} \beta^{2} \sigma_{e e}^{-1}\right)^{2}}\right\} \\
= & \frac{-r_{t} \sigma_{u u}^{-1} \sigma_{e e}^{-2}}{\left(r_{t} \sigma_{u u}^{-1}+s_{t} \beta^{2} \sigma_{e e}^{-1}\right)^{2}} \times\left[s_{t} \beta \sum_{l=1}^{s_{t}}\left(Y_{t l}-\beta \bar{X}_{t .}-\alpha\right)\right. \\
& \left.+s_{t} \sum_{l=1}^{s_{t}}\left(\beta Y_{t l}+\frac{r_{t}}{s_{t}} \cdot \alpha \bar{X}_{t .}-\beta \alpha\right)\right] \\
q_{t \alpha \sigma_{u u}}= & \frac{\partial q_{t \alpha}}{\partial \sigma u u}=\frac{-r_{t} s_{t} \beta^{2} \sigma_{e e}^{-2} \sigma_{u u}^{-2}}{\left(r_{t} \sigma_{u u}^{-1}+s_{t} \beta^{2} \sigma_{e e}^{-1}\right)^{2}} \times \sum_{l=1}^{s_{t}}\left(Y_{t l}-\beta \bar{X}_{t .}-\alpha\right) \\
q_{t \alpha \sigma_{e e}}= & \frac{\partial q_{t \alpha}}{\partial \sigma e e}=\frac{-r_{t}^{2} \sigma_{u u}^{-2} \sigma_{e e}^{-2}}{\left(r_{t} \sigma_{u u}^{-1}+s_{t} \beta^{2} \sigma_{e e}^{-1}\right)^{2}} \times \sum_{l=1}^{s_{t}}\left(Y_{t l}-\beta \bar{X}_{t .}-\alpha\right)
\end{aligned}
$$

(ii) A seguir calcularemos $q_{t \beta l}, l=\alpha, \beta, \sigma_{u u}, \sigma_{e e}$. Temos

$$
\begin{gathered}
q_{t \beta \alpha}=\frac{\partial q_{t \beta}}{\partial \alpha}=\frac{\partial q_{t \alpha}}{\partial \beta} \\
q_{t \beta \beta}=\frac{-r_{t} \sigma_{u u}^{-1} \sigma_{e e}^{-1}\left(4 s_{t} \beta \sigma_{e e}^{-1}\right)}{\left(r_{t} \sigma_{u u}^{-1}+s_{t} \beta^{2} \sigma_{e e}^{-1}\right)^{3}}\left[\sum_{l=1}^{s_{t}}\left(Y_{t l}-\beta \bar{X}_{t .}-\alpha\right)\right] \times\left[\sum_{l=1}^{s_{t}}\left(\beta Y_{t l}+\frac{r_{t}}{s_{t}} \lambda \bar{X}_{t .}-\beta \alpha\right)\right] \\
+\frac{r_{t} \sigma_{u u}^{-1} \sigma_{e e}^{-1}}{\left(r_{t} \sigma_{u u}^{-1}+s_{t} \beta^{2} \sigma_{e e}^{-1}\right)^{2}} \times\left[-s_{t} \bar{X}_{t .} \sum_{l=1}^{s_{t}}\left(\beta Y_{t l}+\frac{r_{t}}{s_{t}} \lambda \bar{X}_{t .}-\beta \alpha\right)\right] \\
+\left[\sum_{l=1}^{s_{t}}\left(Y_{t l}-\alpha\right)\right] \times\left[\sum_{l=1}^{s_{t}}\left(Y_{t l}-\beta \bar{X}_{t .}-\alpha\right)\right]
\end{gathered}
$$




$$
\begin{aligned}
& =\frac{-4 s_{t} \beta \sigma_{e e}^{-1}}{\left(r_{t} \sigma_{u u}^{-1}+s_{t} \beta^{2} \sigma_{e e}^{-1}\right)} \times q_{t \beta}-\frac{s_{t} \sigma_{u u}^{-1} \sigma_{e e}^{-1}}{\left(r_{t} \sigma_{u u}^{-1}+s_{t} \beta^{2} \sigma_{e e}^{-1}\right)^{2}} \times\left(\sum_{j=1}^{r_{t}} X_{t j}\right) \\
& \times\left[\sum_{l=1}^{s_{t}}\left(\beta Y_{t l}+\frac{r_{t}}{s_{t}} \lambda \bar{X}_{t .}-\beta \alpha\right)\right]+\frac{r_{t} \sigma_{u u}^{-1} \sigma_{e e}^{-2}}{\left(r_{t} \sigma_{u u}^{-1}+s_{t} \beta^{2} \sigma_{e e}^{-1}\right)^{2}}\left[\sum_{l=1}^{s_{t}}\left(Y_{t j}-\alpha\right)\right] \\
& \times\left[\sum_{l=1}^{s_{t}}\left(Y_{t l}-\beta \bar{X}_{t .}-\alpha\right)\right] ; \\
& q_{t \beta \sigma_{u u}}=\frac{\partial q_{t \beta}}{\partial \sigma_{u u}}=\frac{r_{t} \beta \sigma_{e e}^{-2} \sigma_{u u}^{-2}}{\left(r_{t} \sigma_{u u}^{-1}+s_{t} \beta^{2} \sigma_{e e}^{-1}\right)^{3}}\left\{r_{t} \sigma_{u u}^{-1} \times\left[\sum_{l=1}^{s_{t}}\left(\beta Y_{t l}-\beta \bar{X}_{t .}-\alpha\right)\right]^{2}\right. \\
& \left.-s_{t} \beta \sigma_{e e}^{-1}\left[\sum_{l=1}^{s_{t}}\left(Y_{t l}-\beta \bar{X}_{t .}-\alpha\right)\right]\left[\sum_{l=1}^{s_{t}}\left(\beta Y_{t l}+\frac{r_{t}}{s_{t}} \lambda \bar{X}_{t .}-\beta \alpha\right)\right]\right\} \\
& =\frac{r_{t} \beta \sigma_{e e}^{-2} \sigma_{u u}^{-1}}{\left(r_{t} \sigma_{u u}^{-1}+s_{t} \beta^{2} \sigma_{e e}^{-1}\right)^{3}}\left[\sum_{l=1}^{s_{t}}\left(Y_{t j}-\beta \bar{X}_{t .}-\alpha\right)\right]^{2}-\frac{s_{t} \beta^{2} \sigma_{u u}^{-1} \sigma_{e e}^{-1}}{\left(r_{t} \sigma_{u u}^{-1}+s_{t} \beta^{2} \sigma_{e e}^{-1}\right)} \times q_{t \beta} ; \\
& q_{t \beta \sigma_{e e}}=\frac{\partial q_{t \beta}}{\partial \sigma_{e e}}=\frac{\left(r_{t} \sigma_{u u}^{-1}\right)\left(r_{t} \sigma_{u u}^{-1} \sigma_{e e}^{-2}\right)}{\left(r_{t} \sigma_{u u}^{-1}+s_{t} \beta^{2} \sigma_{e e}^{-1}\right)^{3}}\left[\sum_{l=1}^{s_{t}}\left(Y_{t l}-\beta \bar{X}_{t .}-\alpha\right)\right] \\
& \times\left\{-2 \sigma_{e e}^{-1} \sum_{l=1}^{s_{t}}\left(\beta Y_{t l}-\frac{r_{t}}{s_{t}} \lambda \bar{X}_{t .}-\beta \alpha\right)+\left(r_{t} \sigma_{u u}^{-1}+s_{t} \beta^{2} \sigma_{e e}^{-1} \bar{X}_{t .}\right\}\right. \\
& =\frac{-r_{t}^{2} \sigma_{u u}^{-2} \sigma_{e e}^{-3}}{\left(r_{t} \sigma_{u u}^{-1}+s_{t} \beta^{2} \sigma_{e e}^{-1}\right)^{3}}\left\{\beta\left[\sum_{l=1}^{s_{t}}\left(Y_{t j}-\beta \bar{X}_{t .}-\alpha\right)\right]^{2}\right. \\
& \left.+\left[\sum_{l=1}^{s_{t}}\left(Y_{t j}-\beta \bar{X}_{t .}-\alpha\right)\right]\left[\sum_{l=1}^{s_{t}}\left(\beta Y_{t j}+\frac{r_{t}}{s_{t}} \lambda \bar{X}_{t .}-\beta \alpha\right)\right]\right\} .
\end{aligned}
$$

(iii) Cálculos de $q_{t \sigma_{u u} l}, l=\alpha, \beta, \sigma_{u u}, \sigma_{e e}$

$$
q_{t \sigma_{u u} \alpha}=q_{t \alpha \sigma_{u u}}
$$




$$
\begin{aligned}
q_{t \sigma_{u u} \beta}= & q_{t \beta \sigma_{u u}} ; \\
q_{t \sigma_{u u} \sigma_{u u}}= & \frac{\partial q_{t \sigma_{u u}}}{\partial \sigma_{u u}}=\frac{r_{t}}{2} \sigma_{u u}^{-2}-\sigma_{u u}^{-3} \sum_{j=1}^{r_{t}}\left(X_{t j}-\bar{X}_{t .}\right)^{2} \\
& -\frac{r_{t} s_{t} \beta^{4} \sigma_{e e}^{-3} \sigma_{u u}^{-3}}{\left(r_{t} \sigma_{u u}^{-1}+s_{t} \beta^{2} \sigma_{e e}^{-1}\right)^{3}}\left[\sum_{l=1}^{s_{t}}\left(Y_{t j}-\beta \bar{X}_{t .}-\alpha\right)\right]^{2} ; \\
q_{t \sigma_{u u} \sigma_{e e}}= & \frac{\partial q_{t \sigma_{u u}}}{\partial \sigma_{e e}}=\frac{r_{t}^{2} \beta^{2} \sigma_{u u}^{-3} \sigma_{e e}^{-3}}{\left(r_{t} \sigma_{u u}^{-1}+s_{t} \beta^{2} \sigma_{e e}^{-1}\right)^{3}}\left[\sum_{l=1}^{s_{t}}\left(Y_{t j}-\beta \bar{X}_{t .}-\alpha\right)\right]^{2} .
\end{aligned}
$$

(iv) Cálculos de $q_{t \sigma_{e e} l}, l=\alpha, \beta, \sigma_{u u}, \sigma_{e e}$

$$
\begin{aligned}
q_{t \sigma_{e e} l}= & q_{t l \sigma_{e e}} ; \quad \text { para } \quad l=\alpha, \beta, \sigma_{u u} \\
q_{t \sigma_{e e} \sigma_{e e}}= & -\frac{s_{t}}{2} \sigma^{-2}-\sigma_{e e}^{-3}\left[\sum_{l=1}^{s_{t}}\left(Y_{t j}-\bar{Y}_{t .}\right)^{2}-\frac{r_{t}^{3} \sigma_{u u}^{-3} \sigma_{e e}^{-3}}{s_{t}\left(r_{t} \sigma_{u u}^{-1}+s_{t} \beta^{2} \sigma_{e e}^{-1}\right)^{3}}\right. \\
& \times\left[\sum_{l=1}^{s_{t}}\left(Y_{t j}-\beta \bar{X}_{t .}-\alpha\right)\right]^{2} .
\end{aligned}
$$

(v) Cálculos de $\left.E\left(q_{t k l}\right)\right|_{\theta=\theta^{1}}$, para $k, l=\alpha, \beta, \sigma_{u u} \cdot \sigma_{e e}$ em que

$$
\theta^{1}=\left[\alpha^{0}, \quad \beta^{0}, \quad\left(\frac{\bar{s}+\bar{r}-1}{\bar{s}+\bar{r}}\right) \sigma_{u u}^{0}, \quad\left(\frac{\bar{s}+\bar{r}-1}{\bar{s}+\bar{r}}\right) \sigma_{e e}^{0}\right] .
$$

De (4.66) a (4.78), obtemos

$$
\begin{aligned}
\left.E^{o}\left(q_{t \alpha \alpha}\right)\right|_{\theta=\theta^{1}} & =\left[-r_{t} s_{t} \frac{1}{r_{t} \sigma_{e e}^{(1)}+s_{t} \beta^{2} \sigma_{u u}^{(1)}}\right] \\
& =-\frac{r_{t} s_{t}}{r_{t}\left(\frac{\bar{s}+\bar{r}-1}{\bar{s}+\bar{r}}\right) \sigma_{e e}^{0}+s_{t} \beta^{2}\left(\frac{\bar{s}+\bar{r}-1}{\bar{s}+\bar{r}}\right) \sigma_{e e}^{0}}
\end{aligned}
$$




$$
\begin{aligned}
= & -\frac{r_{t} s_{t}(\bar{s}+\bar{r})}{r_{t}(\bar{s}+\bar{r}-1) \sigma_{e e}^{0}+s_{t}(\bar{s}+\bar{r}-1) \sigma_{u u}^{0} \beta^{02}} \\
E^{o}\left(q_{t \alpha \beta}\right)= & -\frac{r_{t} \sigma_{u u}^{-1} \sigma_{e e}^{-2}}{\left(r_{t} \sigma_{u u}^{-1}+s_{t} \beta^{2} \sigma_{e e}^{-1}\right)^{2}}\left\{s _ { t } \beta E ^ { 0 } \left[\sum_{l=1}^{s_{t}}\left(Y_{t l}-\beta \bar{X}_{t .}-\alpha\right)\right.\right. \\
& \left.\left.+s_{t} \sum_{l=1}^{s_{t}}\left(\beta Y_{t l}+\frac{r_{t}}{s_{t}} \lambda \bar{X}_{t .}-\beta \alpha\right)\right]\right\}
\end{aligned}
$$

Mas

$$
\left.E^{0}\left(Y_{t l}-\beta \bar{X}_{t .}-\alpha\right)\right|_{\theta(1)}=\alpha^{0}+\beta^{0} x_{t}^{0}-\beta^{0} x_{t}^{0}-\alpha^{0}=0
$$

e

$$
\left.E^{0}\left(\beta Y_{t l}+\frac{r_{t}}{s_{t}} \lambda \bar{X}_{t .}-\beta \alpha\right)\right|_{\theta=\theta^{(1)}}=x_{t}^{0}\left(\beta^{02}+\lambda \frac{r_{t}}{s_{t}}\right) .
$$

De (4.81) e (4.82), temos

$$
\begin{aligned}
\left.E^{0}\left(q_{t \alpha \beta}\right)\right|_{\theta=\theta^{(1)}} & =-\frac{r_{t}\left(\frac{\bar{r}+\bar{s} t}{\bar{r}+\bar{s}-1}\right)^{3}\left(\sigma_{u u}^{0}\right)^{-1}\left(\sigma_{e e}^{0}\right)^{-2}}{\left(\frac{\bar{r}+\bar{s}}{\bar{r}+\bar{s}-1}\right)^{2}\left[r_{t}\left(\sigma_{u u}^{0}\right)^{-1}+s_{t} \beta^{02}\left(\sigma_{e e}^{0}\right)^{-1}\right]^{2}}\left[s_{t}^{2}\left(x_{t}^{0}\right)\left(\beta^{02}+\lambda^{0} \frac{r_{t}}{s_{t}}\right)\right] \\
& =-r_{t}\left(\frac{\bar{r}+\bar{s}}{\bar{r}+\bar{s}-1}\right) \frac{\left(\sigma_{u u}^{0}\right)^{-1}\left(\sigma_{e e}^{0}\right)^{-2}}{s_{t}^{2}\left(\sigma_{e e}^{0}\right)^{-2}\left(\frac{r_{t}}{s_{t}} \lambda^{0}+\beta^{02}\right)^{2}}\left[s_{t}^{2}\left(x_{t}^{0}\right)\left(\beta^{02}+\lambda^{0} \frac{r_{t}}{s_{t}}\right)\right] \\
& =-r_{t}\left(\frac{\bar{r}+\bar{s}}{\bar{r}+\bar{s}-1}\right) \frac{\left(\sigma_{u u}^{0}\right)^{-1}\left(x_{t}^{0}\right)}{\left(\frac{r_{t}}{s_{t}} \lambda^{0}+\beta^{02}\right)} \\
& =-r_{t}\left(\frac{\bar{r}+\bar{s}}{\bar{r}+\bar{s}-1}\right) \frac{s_{t}\left(\sigma_{u u}^{0}\right)^{-1}\left(x_{t}^{0}\right)}{\left(r_{t} \lambda^{0}+s_{t} \beta^{02}\right)} \\
& =-\frac{\bar{r}+\bar{s}}{\bar{r}+\bar{s}-1} \frac{s t r_{t} x_{t}^{0}\left(\sigma_{u u}^{0}\right)^{-1}}{\left(r_{t} \lambda^{0}+\beta^{02}\right)} .
\end{aligned}
$$

De (4.70), temos

$$
E^{0}\left(q_{t \beta \beta}\right)=-\left.\frac{r_{t} s_{t} x_{t}^{02} \sigma_{u u}^{-1}}{\left(r_{t} \lambda^{0}+s_{t} \beta^{02}\right)} \Longrightarrow\left[E^{0}\left(q_{t \beta \beta}\right)\right]\right|_{\theta^{1}}=-\frac{r_{t} s_{t} x_{t}^{02}}{\left(r_{t} \lambda^{0}+s_{t} \beta^{02}\right)} \frac{(\bar{r}+\bar{s})\left(\sigma_{u u}^{0}\right)^{-1}}{(\bar{r}+\bar{s}-1)}
$$

De (4.71) e (4.72), obtemos 


$$
\begin{aligned}
E^{0}\left(q_{t \beta \sigma_{u u}}\right) & =\frac{r_{t} s_{t} \lambda \beta^{0} \sigma_{u u}^{0}}{\sigma_{u u}^{2}\left(r_{t} \lambda^{0}+s_{t} \beta^{02}\right)^{2}} ; \\
E^{0}\left(q_{t \beta \sigma_{e e}}\right) & =-\frac{r_{t} s_{t} \beta^{0} \sigma_{u u}^{0}}{\sigma_{u u}^{2}\left(r_{t} \lambda^{0}+s_{t} \beta^{02}\right)^{2}} ; \\
E^{0}\left(q_{t \sigma_{u u} \sigma_{u u}}\right) & =\frac{r_{t}}{2} \sigma_{u u}^{-2}-\sigma_{u u}^{-3}\left(r_{t}-1\right) \sigma_{u u}^{0}-\frac{s_{t}^{2} \sigma_{u u}^{-3} \beta^{04} \sigma_{u u}^{0}}{\left(r_{t} \lambda^{0}+s_{t} \beta^{02}\right)^{2}} ; \\
\left.E^{0}\left(q_{t \sigma_{u u} \sigma_{e e}}\right)\right|_{\theta=\theta^{1}} & =-\left.\frac{r_{t} s_{t} \beta^{2} \sigma_{\delta \delta}^{-3}}{\left(r_{t} \lambda+s_{t} \beta^{2}\right)^{2}} \sigma_{u u}^{0}\right|_{\theta=\theta^{1}}=-\frac{r_{t} s_{t} \beta^{02} \sigma_{u u}^{-2}}{\left(r_{t} \lambda^{0}+s_{t} \beta^{02}\right)^{2}}\left(\frac{\bar{r}+\bar{s}}{\bar{r}+\bar{s}-1}\right)^{3} ; \\
E^{0}\left(q_{t \sigma_{e e} \sigma_{e e}}\right) & =\frac{s_{t}}{2} \sigma_{e e}^{-2}-\sigma_{e e}^{-3}\left(s_{t}-1\right) \sigma_{e e}^{0}-\frac{r_{t}^{3} \sigma_{e e}^{-3}}{s_{t}} \frac{s_{t} \sigma_{u u}^{0}\left(r_{t} \lambda+\beta^{02} s_{t}\right)}{\left(r_{t} \lambda+s_{t} \beta^{2}\right)^{3} r_{t}}\left(\sigma_{e e}^{0}\right)^{-2}\left(\sigma_{e e}^{0}\right)^{-2} .
\end{aligned}
$$

Cálculos de $E\left[\left(A_{n}\left(\theta^{1}\right)\right)_{k l}\right]$

De (4.79) e (4.88), obtemos:

$$
\begin{aligned}
E^{o}\left[\left(A_{n}\left(\theta^{1}\right)\right)_{11}\right] & =\left.\left[E^{o}\left(\frac{\sum_{t=1}^{n} q_{t \alpha \alpha}}{n}\right)\right]\right|_{\theta=\theta^{(1)}} \\
& =-\frac{(\bar{r}+\bar{s})}{n(\bar{s}+\bar{r}-1) \sigma_{u u}^{0}} \sum_{t=1}^{n}\left(\frac{r_{t} s_{t}}{r_{t} \lambda^{0}+s_{t}\left(\beta^{0}\right)^{2}}\right) ; \\
E^{0}\left[\left(A_{n}\left(\theta^{1}\right)\right)_{12}\right] & =\left.\frac{1}{n}\left[\sum_{t=1}^{n} E^{0}\left(q_{t \alpha \beta}\right)\right]\right|_{\theta=\theta^{(1)}}=-\frac{(\bar{r}+\bar{s})}{n(\bar{s}+\bar{r}-1) \sigma_{u u}^{0}} \sum_{t=1}^{n} \frac{s_{t} r_{t} x_{t}^{0}}{\left(r_{t} \lambda^{0}+s_{t}\left(\beta^{0}\right)^{2}\right)} ; \\
E^{0}\left[\left(A_{n}\left(\theta^{1}\right)\right)_{13}\right] & =\left.\left[\sum_{t=1}^{n} \frac{E^{0}\left(q_{t \alpha \sigma_{u u}}\right)}{n}\right]\right|_{\theta=\theta^{1}}=0 ; \\
E^{0}\left[\left(A_{n}\left(\theta^{1}\right)\right)_{14}\right] & =\left.\left[\sum_{t=1}^{n} \frac{E^{0}\left(q_{t \alpha \sigma_{e e}}\right)}{n}\right]\right|_{\theta=\theta^{1}}=0 ; \\
E^{0}\left[\left(A_{n}\left(\theta^{1}\right)\right)_{22}\right] & =\left.\left[\sum_{t=1}^{n} \frac{E^{0}\left(q_{t \beta \beta}\right)}{n}\right]\right|_{\theta=\theta^{1}}=-\frac{\bar{r}+\bar{s}}{n \sigma_{u u}^{0}(\bar{r}+\bar{s}-1)} \sum_{t=1}^{n} \frac{r_{t} s_{t} x_{t}^{02}}{\left(r_{t} \lambda^{0}+s_{t}\left(\beta^{0}\right)^{2}\right)}
\end{aligned}
$$




$$
\begin{aligned}
& E^{0}\left[\left(A_{n}\left(\theta^{1}\right)\right)_{23}\right]=\left.\left[\frac{\sum_{t=1}^{n} E^{0}\left(q_{t \beta \sigma_{u u}}\right)}{n}\right]\right|_{\theta=\theta^{(1)}} \\
& =\frac{\lambda^{0}+\beta^{0}\left(\sigma_{u u}^{0}\right)^{-1}}{n}\left(\frac{\bar{r}+\bar{s}}{\bar{r}+\bar{s}-1}\right)^{2} \sum_{t=1}^{n} \frac{r_{t} s_{t}}{\left(r_{t} \lambda^{0}+s_{t}\left(\beta^{0}\right)^{2}\right)^{2}} \text {; } \\
& E^{0}\left[\left(A_{n}\left(\theta^{1}\right)\right)_{24}\right]=\left.\left[\sum_{t=1}^{n} \frac{E^{0}\left(q_{t \beta \sigma_{e e}}\right)}{n}\right]\right|_{\theta=\theta^{1}}=-\frac{\beta^{0}\left(\sigma_{u u}^{0}\right)^{-1}}{n}\left(\frac{\bar{r}+\bar{s}}{\bar{r}+\bar{s}-1}\right)^{2} \sum_{t=1}^{n} \frac{r_{t} s_{t}}{\left(r_{t} \lambda^{0}+s_{t} \beta^{02}\right)^{2}} \\
& E^{0}\left[\left(A_{n}\left(\theta^{1}\right)\right)_{33}\right]=\left.\left[\frac{\sum_{t=1}^{n} E^{0}\left(q_{t \sigma_{u u} \sigma_{u u}}\right)}{n}\right]\right|_{\theta=\theta^{(1)}} \\
& =\frac{1}{n}\left(\frac{\bar{r}+\bar{s}}{\bar{r}+\bar{s}-1}\right)^{3}\left(\sigma_{u u}^{0}\right)^{-2}\left\{\left(\frac{\bar{r}+\bar{s}-1}{\bar{r}+\bar{s}}\right) \frac{\sum_{t=1}^{n} r_{t}}{2}-\sum_{t=1}^{n}\left(r_{t}-1\right)\right. \\
& \left.-\beta^{04} \sum_{t=1}^{n} \frac{s_{t}^{2}}{\left(r_{t} \lambda^{0}+s_{t} \beta^{02}\right)^{2}}\right\} \text {; } \\
& E^{0}\left[\left(A_{n}\left(\theta^{1}\right)\right)_{34}\right]=\left.\left[\frac{\sum_{t=1}^{n} E^{0}\left(q_{t \sigma_{u u} \sigma_{e e}}\right)}{n}\right]\right|_{\theta=\theta^{(1)}} \\
& =-\frac{\beta^{02}\left(\sigma_{u u}^{0}\right)^{-2}(\bar{r}+\bar{s})^{3}}{n(\bar{r}+\bar{s}-1)^{3}} \sum_{t=1}^{n} \frac{r_{t} s_{t}}{\left(r_{t} \lambda^{0}+s_{t} \beta^{02}\right)^{2}} ; \\
& E^{0}\left[\left(A_{n}\left(\theta^{1}\right)\right)_{44}\right]=\left.\left[\frac{\sum_{t=1}^{n} E^{0}\left(q_{t \sigma_{e e} \sigma_{e e}}\right)}{n}\right]\right|_{\theta=\theta(1)} \\
& =\frac{1}{n}\left(\frac{\bar{r}+\bar{s}}{\bar{r}+\bar{s}-1}\right)^{3}\left(\sigma_{e e}^{0}\right)^{-2}\left\{\frac{\bar{r}+\bar{s}-1}{\bar{r}+\bar{s}} \frac{\sum_{t=1}^{n} s_{t}}{2}\right. \\
& \left.-\sum_{t=1}^{n}\left(s_{t}-1\right)-\sum_{t=1}^{n} \frac{r_{t}^{2} \lambda^{2}}{\left(r_{t} \lambda+s_{t} \beta^{02}\right)^{2}}\right\} \text {. }
\end{aligned}
$$

De (4.89) - (4.98), podemos escrever $E^{0}\left(A_{n}\left(\theta^{1}\right)\right)=$ $=\left(\frac{w}{n \sigma_{u u}^{0}}\right)\left[\begin{array}{cccc}-\sum_{t=1}^{n} v_{t} & -\sum_{t=1}^{n} v_{t} x_{t}^{0} & 0 & 0 \\ -\sum_{t=1}^{n} v_{t} x_{t}^{0} & -\sum_{t=1}^{n} v_{t} x_{t}^{0^{2}} & \lambda^{0} w \beta^{0} \Sigma_{r s} & -\beta^{0} w \Sigma_{r s} \\ 0 & -\lambda \beta^{0} w \Sigma_{r s} & \frac{w^{2}}{\sigma_{u u}^{0}}\left(n c-\beta^{04} \Sigma_{s s}\right) & \frac{-\beta^{0} w^{2}}{\sigma_{u u}} \Sigma_{r s} \\ 0 & -\beta^{0} w \Sigma_{r s} & \frac{-w^{2} \beta^{02}}{\sigma_{u u}} \Sigma_{r s} & \frac{w^{2}}{\lambda^{2} \sigma_{u u}^{0}}\left(n b-\lambda^{2} \Sigma_{r r}\right)\end{array}\right]$ 
onde

$$
\begin{aligned}
& v_{t}=\frac{r_{t} s_{t}}{\Delta_{t}} ; \quad \Delta_{t}=r_{t} \lambda+s_{t} \beta^{0^{2}} ; \quad \Sigma_{r s}=\sum_{t=1}^{n} \frac{r_{t} s_{t}}{\Delta_{t}^{2}} ; \quad \Sigma_{r r}=\sum_{t=1}^{n} \frac{r_{t}^{2}}{\Delta_{t}^{2}} ; \quad \Sigma_{s s}=\sum_{t=1}^{n} \frac{s_{t}^{2}}{\Delta_{t}^{2}} ; \\
& \bar{\Sigma}_{r s}=\frac{\Sigma_{r s}}{n} ; \quad c=\frac{\bar{r}}{2 w}-\bar{r}+1 ; \quad b=\frac{\bar{s}}{2 w}-\bar{s}+1 ; \quad w=\frac{\bar{r}+\bar{s}}{\bar{r}+\bar{s}-1} .
\end{aligned}
$$

\subsubsection{Cálculo de $V_{n}$}

Como mencionado acima

$$
\begin{aligned}
V_{n} & =\sum_{t=1}^{n} \frac{\operatorname{cov}\left(q_{t}\left[\left(X_{t}, Y_{t}\right), \theta^{1}\right] / \theta^{0}, x_{t}^{0}\right)}{n} \\
& =\frac{\sum_{t=1}^{n} \operatorname{cov}^{0}\left[q_{t}\left(\theta^{1}\right)\right]}{n}
\end{aligned}
$$

em que

$$
q_{t}\left(\theta^{1}\right)=\left\{q_{t \alpha}\left(\theta^{1}\right), q_{t \beta}\left(\theta^{1}\right), q_{t \sigma_{u u}}\left(\theta^{1}\right), q_{t \sigma_{e e}}\left(\theta^{1}\right)\right\} .
$$

Após algumas manipulações algébricas, obtemos

$$
\begin{aligned}
V_{n 11} & =\left[\frac{1}{n} \sum_{t=1}^{n} \operatorname{cov}^{0}\left(q_{t \alpha}, q_{t \alpha}\right)\right]_{\theta=\theta^{(1)}}=\frac{1}{n} \sum_{t=1}^{n}\left[\frac{\sigma_{u u}^{-2} s_{t} r_{t} \sigma_{u u}^{0}}{\left(r_{t} \lambda+s_{t} \beta^{02}\right)}\right]_{\theta=\theta^{(1)}} \\
& =\frac{1}{n}(w)^{2}\left(\sigma_{u u}^{0}\right)^{-1} \sum_{t=1}^{n} \frac{s_{t} r_{t}}{\Delta_{t}}=\frac{w^{2}}{n \sigma_{u u}^{0}} \sum_{t=1}^{n} \frac{s_{t} r_{t}}{\Delta_{t}} ; \\
V_{n 12} & =\left[\frac{1}{n} \sum_{t=1}^{n} \operatorname{cov}^{0}\left(q_{t \alpha}, q_{t \beta}\right)\right]_{\theta=\theta^{(1)}}=\left[\frac{1}{n} \frac{r_{t} s_{t} \sigma_{u u}^{-2}}{\left(r_{t} \lambda+s_{t} \beta^{02}\right)} x_{t}^{0} \sigma_{u u}^{0}\right]_{\theta=\theta^{(1)}} \\
& =\left(\frac{1}{n \sigma_{u u}^{0}}\right)\left(\frac{\bar{r}+\bar{s}}{\bar{r}+\bar{s}-1}\right)^{2} \sum_{t=1}^{n} \frac{s_{t} r_{t} x_{t}^{0}}{\left(r_{t} \lambda+s_{t} \beta^{02}\right)}=\frac{1}{n \sigma_{u u}^{0}} w^{2} \sum_{t=1}^{n} \frac{s_{t} r_{t} x_{t}^{0}}{\Delta_{t}} \\
V_{n 13} & =\left[\frac{1}{n} \sum_{t=1}^{n} \operatorname{cov}^{0}\left(q_{t \alpha}, q_{t \sigma_{u u}}\right)\right]_{\theta=\theta^{(1)}}=0 \\
V_{n 14} & =\left[\frac{1}{n} \sum_{t=1}^{n} \operatorname{cov}^{0}\left(q_{t \alpha}, q_{t \sigma_{e e}}\right)\right]_{\theta=\theta^{(1)}}=0
\end{aligned}
$$




$$
\begin{aligned}
& V_{n 22}=\left.\left[\frac{1}{n} \sum_{t=1}^{n} \operatorname{cov}^{0}\left(q_{t \beta}, q_{t \beta}\right)\right]\right|_{\theta=\theta^{(1)}} \\
& =\frac{1}{n} \sum_{t=1}^{n}\left\{\frac{r_{t} \sigma_{e e}^{-2} s_{t} \sigma_{e e}^{0} x_{t}^{0}}{\left(r_{t} \lambda+s_{t} \beta^{02}\right)}+\frac{r_{t} \sigma_{e e}^{-2} s_{t} \sigma_{e e}^{0} \sigma_{u u}^{0}}{\left(r_{t} \lambda+s_{t} \beta^{02}\right)^{2}}\right\} \\
& =\frac{1}{n}\left\{w^{2}\left(\sigma_{u u}^{0}\right)^{-1} \sum_{t=1}^{n} \frac{s_{t} r_{t} x_{t}^{02}}{\Delta_{t}}+w^{2}\left(\sigma_{u u}^{0}\right)^{-1} \sigma_{e e}^{0} \sum_{t=1}^{n} \frac{s_{t} r_{t}}{\Delta t^{2}}\right\} \\
& =\frac{w^{2}}{n \sigma_{u u}^{0}}\left\{\sum_{t=1}^{n} \frac{s_{t} r_{t} x_{t}^{02}}{\Delta_{t}}+\sigma_{e e}^{0} \sum_{t=1}^{n} \frac{s_{t} r_{t}}{\Delta_{t}}\right\} \\
& V_{n 23}=\left.\left[\frac{1}{n} \sum_{t=1}^{n} \operatorname{cov}^{0}\left(q_{t \beta}, q_{t \sigma_{u u}}\right)\right]\right|_{\theta=\theta^{(1)}}=0 \\
& V_{n 24}=\left.\left[\frac{1}{n} \sum_{t=1}^{n} \operatorname{cov}^{0}\left(q_{t \beta}, q_{t \sigma_{e e}}\right)\right]\right|_{\theta=\theta^{(1)}}=0 \\
& V_{n 33}=\left.\left[\frac{1}{n} \sum_{t=1}^{n} \operatorname{cov}^{0}\left(q_{t \sigma_{u u}}, q_{t \sigma_{u u}}\right)\right]\right|_{\theta=\theta^{(1)}}=\frac{w^{4}}{2 n\left(\sigma_{u u}^{0}\right)^{4}} \sum_{t=1}^{n}\left\{\frac{s_{t}^{2} \beta^{04} \sigma_{u u}^{02}}{\left(r_{t} \lambda+s_{t} \beta^{02}\right)^{2}}+\left(r_{t}-1\right) \sigma_{u u}^{02}\right\} \\
& =\frac{1}{2 n} \frac{w^{4}}{\sigma_{u u}^{02}}\left\{\beta^{04} \sum_{t=1}^{n} \frac{s_{t}^{2}}{\Delta_{t}^{2}}+\sum_{t=1}^{n}\left(r_{t}-1\right)\right\} \\
& V_{n 34}=\left.\left[\frac{1}{n} \sum_{t=1}^{n} \operatorname{cov}^{0}\left(q_{t \sigma_{u u}}, q_{t \sigma_{e e}}\right)\right]\right|_{\theta=\theta^{(1)}}=\frac{1}{n} \sum_{t=1}^{n}\left\{\frac{r_{t} s_{t} \beta^{02} \sigma_{u u}^{-4} \sigma_{u u}^{02}}{2\left(r_{t} \lambda+s_{t} \beta^{02}\right)^{2}}\right\} \\
& =\frac{1}{2 n} \frac{w^{4} \beta^{04}}{\sigma_{u u}^{02}} \sum_{t=1}^{n} \frac{s_{t} r_{t}}{\Delta_{t}^{2}} \\
& \text { e } \\
& V_{n 44}=\left.\left[\frac{1}{n} \sum_{t=1}^{n} \operatorname{cov}^{0}\left(q_{t \sigma_{e e}}, q_{t \sigma_{e e}}\right)\right]\right|_{\theta=\theta(1)} \\
& =\frac{1}{n} \sum_{t=1}^{n}\left\{\frac{1}{2} \sigma_{e e}^{-4}\left[\left(s_{t}-1\right) \sigma_{e e}^{02}+\frac{r_{t}^{2} \sigma_{u u}^{02} \lambda^{4}}{\left(r_{t} \lambda+s_{t} \beta^{02}\right)^{2}}\right]\right\} \text {. }
\end{aligned}
$$


De (4.101) a (4.109), podemos construir a matriz $V_{n}$ como

$$
\begin{gathered}
V_{n}=\left(\frac{1}{n \sigma_{u u}^{0}}\right) \times \\
{\left[\begin{array}{cccc}
w^{2} \sum_{t=1}^{n} v_{t} & w^{2} \sum_{t=1}^{n} v_{t} x_{t}^{0} & 0 & 0 \\
w^{2} \sum_{t=1}^{n} v_{t} x_{t}^{0} & w^{2} \sum_{t=1}^{n} v_{t}\left(x_{t}^{0}+\frac{\lambda^{0} \sigma_{u u}^{0}}{\Delta_{t}}\right) & 0 & \\
0 & 0 & \frac{w^{4}}{2 \sigma_{u u}^{0}}\left(n(\bar{r}-1)+\beta^{04} \Sigma_{s s}\right) & \frac{\beta^{0} w^{4}}{2 \sigma_{u u}} \Sigma_{r s} \\
0 & 0 & \frac{\beta^{0} w^{4}}{2 \sigma_{u u}} \Sigma_{r s} & \frac{w^{4}}{2 \lambda^{2} \sigma_{u u}^{0}}\left(n(\bar{s}-1)+\lambda^{2} \Sigma_{r r}\right)
\end{array}\right] .}
\end{gathered}
$$

Finalmente, depois de exaustivas manipulações algébricas, a matriz de covariâncias assintóticas do estimador de máxima verossimilhança de $\theta^{0}$ pode ser escrita como

$$
\begin{gathered}
n^{-1}\left\{E^{0}\left(A_{n}\left(\theta^{1}\right)\right)\right\}^{-1} V_{n}\left\{E^{0}\left(A_{n}\left(\theta^{1}\right)\right)\right\}^{-1}=\left(\frac{w^{-1} \sigma_{u u}^{0}}{D_{1}^{2}}\right) \times \\
{\left[\begin{array}{cccc}
w\left(F_{1}+F_{2} B_{2}^{2}\right) & w\left(C_{1} F_{3}-F_{2} B_{1} B_{2}\right) & B_{2}\left(C_{3} F_{5}+C_{4} F_{6}\right)-C_{3} F_{3} & -B_{2}\left(C_{3} F_{7}+C_{4} F_{8}\right)-C_{4} F_{3} \\
w\left(C_{1} F_{3}-F_{2} B_{1} B_{2}\right) & w\left(F_{2} B_{1}^{2}-F_{4} C_{1}\right) & C_{3} F_{4}+B_{2}\left(C_{3} F_{5}+C_{4} F_{6}\right) & B_{1}\left(C_{3} F_{7}+C_{4} F_{8}\right)+C_{4} F_{4} \\
- & - & \frac{1}{w}\left[\sigma_{u u}^{0}\left(D_{3} F_{5}-D_{4} F_{6}\right)-\frac{C_{3}^{2} F_{4}}{C_{1}}\right] & \frac{1}{w}\left[\sigma_{u u}^{0}\left(D_{4} F_{8}-D_{3} F_{7}\right)-\frac{C_{3} C_{4} F_{4}}{C_{2}^{2}}\right] \\
- & - & - & \frac{1}{w}\left[\sigma_{u u}^{0}\left(D_{4} F_{7}-D_{5} F_{8}\right)-\frac{C_{4}^{2} F_{4}}{C_{1}}\right]
\end{array}\right],}
\end{gathered}
$$

onde

$$
\begin{aligned}
v_{t} & =\frac{r_{t} s_{t}}{\Delta_{t}}, \quad \Delta_{t}=\left(r_{t} \lambda+s_{t} \beta^{0^{2}}\right), \quad \lambda=\frac{\sigma_{e e}}{\sigma_{u u}}=\lambda^{0} \frac{\sigma_{e e}^{0}}{\sigma_{u u}^{0}} \\
B_{1} & =-\sum_{t=1}^{n} v_{t}, \quad B_{2}=-\sum_{t=1}^{n} v_{t} x_{t}^{0}, \quad B_{3}=n \bar{r}\left(\frac{1}{2 w}-1\right)+n-\sum_{t=1}^{n} \frac{s_{t}^{2} \beta^{0^{4}}}{\Delta_{t}^{2}} \\
B_{4} & =n \bar{s}\left(\frac{1}{2 w}-1\right)+n-\sum_{t=1}^{n} \frac{r_{t}^{2} \lambda^{2}}{\Delta_{t}^{2}}
\end{aligned}
$$




$$
\begin{aligned}
& C_{1}=\frac{1}{\lambda^{2} \sigma_{u u}^{0}}\left\{B_{3} B_{4}-\left(\sum_{t=1}^{n} \frac{v_{t} \lambda \beta^{0^{2}}}{\Delta_{t}}\right)^{2}\right\}, \quad C_{2}=\left(\sum_{t=1}^{n} v_{t}\right)\left(\sum_{t=1}^{n} v_{t} x_{t}^{02}\right)-\left(\sum_{t=1}^{n} v_{t} x_{t}^{0}\right)^{2}, \\
& C_{3}=\frac{\beta^{0}}{\lambda}\left(\sum_{t=1}^{n} \frac{v_{t}}{\Delta_{t}}\right)\left(B_{4}-\lambda \beta^{02} \sum_{t=1}^{n} \frac{v_{t}}{\Delta_{t}}\right), \quad C_{4}=-\beta^{0}\left(\sum_{t=1}^{n} \frac{v_{t}}{\Delta_{t}}\right)\left(B_{3}-\lambda \beta^{02} \sum_{t=1}^{n} \frac{v_{t}}{\Delta_{t}}\right) \\
& D_{1}=C_{1} C_{2}-\frac{n(\bar{r}+\bar{s}-1)}{2} \beta^{02}\left(\sum_{t=1}^{n} v_{t}\right)\left(\sum_{t=1}^{n} \frac{v_{t}}{\Delta_{t}}\right)^{2} \\
& D_{2}=-C_{1} \sum_{t=1}^{n} v_{t} x_{t}^{02}+\frac{n \beta^{02}(\bar{r}+\bar{s}-1)}{2}\left(\sum_{t=1}^{n} \frac{v_{t}}{\Delta_{t}}\right)^{2} \text {, } \\
& D_{3}=\frac{B_{4} C_{2}}{\lambda^{2} \sigma_{u u}^{0}}+\left(\sum_{t=1}^{n} v_{t}\right)\left(\beta^{0} \sum_{t=1}^{n} \frac{v_{t}}{\Delta_{t}}\right)^{2} \\
& D_{4}=-\left(\beta^{02} \sum_{t=1}^{n} \frac{v_{t}}{\Delta_{t}}\right)\left[\frac{C_{2}}{\sigma_{u u}^{0}}+\lambda\left(\sum_{t=1}^{n} v_{t}\right)\left(\sum_{t=1}^{n} \frac{v_{t}}{\Delta_{t}}\right)\right] \\
& D_{5}=\frac{B_{3} C_{2}}{\sigma_{u u}^{0}}+\left(\sum_{t=1}^{n} v_{t}\right)\left(\lambda \beta^{0} \sum_{t=1}^{n} \frac{v_{t}}{\Delta_{t}}\right)^{2} \\
& F_{1}=D_{2}^{2} \sum_{t=1}^{n} v_{t}+C_{1}\left\{2 D_{2}+C_{1} \sum_{t=1}^{n}\left[v_{t}\left(x_{t}^{02}+\frac{\lambda \sigma_{u u}^{0}}{\Delta_{t}}\right)\right]\right\}\left(\sum_{t=1}^{n} v_{t} x_{t}^{0}\right)^{2} \text {, } \\
& F_{2}=\frac{1}{2 \sigma_{u u}^{0}}\left(\frac{\beta^{0}}{\lambda} \sum_{t=1}^{n} \frac{v_{t}}{\Delta_{t}}\right)^{2}\left\{\left(\frac{n \bar{r}}{2 w}-B_{3}\right)\left(B_{4}-\lambda \beta^{02} \sum_{t=1}^{n} \frac{v_{t}}{\Delta_{t}}\right)^{2}\right. \\
& -\left(2 \lambda \beta^{02} \sum_{t=1}^{n} \frac{v_{t}}{\Delta_{t}}\right)\left(B_{4}-\lambda \beta^{02} \sum_{t=1}^{n} \frac{v_{t}}{\Delta_{t}}\right)\left(B_{3}-\lambda \beta^{02} \sum_{t=1}^{n} \frac{v_{t}}{\Delta_{t}}\right) \\
& \left.+\left(\frac{n \bar{s}}{2 w}-B_{4}\right)\left(B_{3}-\lambda \beta^{02} \sum_{t=1}^{n} \frac{v_{t}}{\Delta_{t}}\right)^{2}\right\} \\
& F_{3}=-C_{1}\left(C_{2}+\left(\sum_{t=1}^{n} v_{t}\right) \lambda \sigma_{u u}^{0} \sum_{t=1}^{n} \frac{v_{t}}{\Delta_{t}}\right) \sum_{t=1}^{n} v_{t} x_{t}^{0} \\
& F_{4}=-C_{1}\left(\sum_{t=1}^{n} v_{t}\right)\left(C_{2}+\left(\sum_{t=1}^{n} v_{t}\right) \lambda \sigma_{u u}^{0} \sum_{t=1}^{n} \frac{v_{t}}{\Delta_{t}}\right) \text {, }
\end{aligned}
$$




$$
\begin{aligned}
F_{5}= & \frac{1}{2}\left(\frac{n \bar{r}}{2 w}-B_{3}\right)\left[\frac{B_{4} C_{2}}{\lambda^{2} \sigma_{u u}^{0}}+\beta^{02}\left(\sum_{t=1}^{n} v_{t}\right)\left(\sum_{t=1}^{n} \frac{v_{t}}{\Delta_{t}}\right)^{2}\right] \\
& +\frac{1}{2}\left(\beta^{02} \sum_{t=1}^{n} \frac{v_{t}}{\Delta_{t}}\right)^{2}\left[\frac{C_{2}}{\sigma_{u u}^{0}}+\lambda\left(\sum_{t=1}^{n} v_{t}\right)\left(\sum_{t=1}^{n} \frac{v_{t}}{\Delta_{t}}\right)\right], \\
F_{6}= & \frac{1}{2}\left(\beta^{02} \sum_{t=1}^{n} \frac{v_{t}}{\Delta_{t}}\right)\left\{( \sum _ { t = 1 } ^ { n } v _ { t } ) ( \sum _ { t = 1 } ^ { n } \frac { v _ { t } } { \Delta _ { t } } ) \left[\beta^{0^{2}} \sum_{t=1}^{n} \frac{v_{t}}{\Delta_{t}}\right.\right. \\
& \left.\left.+\frac{1}{\lambda}\left(\frac{n \bar{s}}{2 w}-B_{4}\right)\right]+\frac{n \bar{s} C_{2}}{2 \lambda^{2} w \sigma_{u u}^{0}}\right\}, \\
F_{7}= & -\frac{1}{2}\left(\beta^{02} \sum_{t=1}^{n} \frac{v_{t}}{\Delta_{t}}\right)\left\{\lambda\left(\sum_{t=1}^{n} v_{t}\right)\left(\sum_{t=1}^{n} \frac{v_{t}}{\Delta_{t}}\right)\left[\left(\frac{n \bar{r}}{2 w}-B_{3}\right)+\lambda \beta^{02} \sum_{t=1}^{n} \frac{v_{t}}{\Delta_{t}}\right]+\frac{n \bar{r} C_{2}}{2 w \sigma_{u u}^{0}}\right\}
\end{aligned}
$$

$\mathrm{e}$

$$
\begin{aligned}
F_{8}= & -\frac{1}{2}\left(\beta^{0} \sum_{t=1}^{n} \frac{v_{t}}{\Delta_{t}}\right)^{2}\left[\left(\frac{\beta^{02} C_{2}}{\sigma_{u u}^{0}}\right)+\sum_{t=1}^{n} v_{t}\left(\lambda \beta^{02} \sum_{t=1}^{n} \frac{v_{t}}{\Delta_{t}}+\frac{n \bar{s}}{2 w}-B_{4}\right)\right] \\
& -\frac{1}{2 \lambda^{2}}\left(\frac{n \bar{s}}{2 w}-B_{4}\right) \frac{B_{3} C_{2}}{\sigma_{u u}^{0}} .
\end{aligned}
$$

\subsection{Avaliações Numéricas}

Apresentamos, nesta Seção, os resultados numéricos nos quais comparamos a eficiência assintótica relativa (EAR) dos estimadores de máxima verossimilhança ( $\left.\widehat{\beta}_{M V}\right)$ obtidos nas Seções anteriores com os dois estimadores propostos em Dorf e Gurland (1960), dados a seguir.

$$
\hat{\beta}_{D G 1}=\frac{B_{X Y}}{B_{X X}-W_{X X}} \quad \text { e } \quad \hat{\beta}_{D G 2}=\frac{B_{Y Y}-W_{y Y}}{B_{X Y}}
$$

onde

$$
B_{X X}=\sum_{t=1}^{n} \frac{r_{t}\left(\bar{X}_{t .}-\bar{X}_{. .}\right)^{2}}{n-1}, \quad W_{X X}=(R-n)^{-1} \sum_{t=1}^{n} \sum_{i=1}^{r_{t}}\left(X_{t i}-\bar{X}_{t .}\right)^{2} \text {, }
$$




$$
B_{Y Y}=\sum_{t=1}^{n} \frac{s_{t}\left(\bar{Y}_{t .}-\bar{Y}_{. .}\right)^{2}}{n-1} \text { e } \quad W_{Y Y}=(S-n)^{-1} \sum_{t=1}^{n} \sum_{l=1}^{s_{t}}\left(X_{t l}-\bar{Y}_{t .}\right)^{2}
$$

com

$$
R=\sum_{t=1}^{n} r_{t} \quad \text { e } \quad S=\sum_{t=1}^{n} s_{t}
$$

As variâncias assintóticas são dadas por

$$
\begin{aligned}
A V A R\left(\hat{\beta}_{D G 1}\right) & =\frac{\beta^{2} \sigma_{u u}}{\sum_{t=1}^{n} r_{t}\left(x_{t}-\bar{x}\right)^{2}}+\frac{\sigma_{e e} \sum_{t=1}^{n}\left(r_{t}^{2} / s_{t}\right)\left(x_{t}-\bar{x}\right)^{2}}{\left(\sum_{t=1}^{n} r_{t}\left(x_{t}-\bar{x}\right)^{2}\right)^{2}} \\
& +\frac{n-1}{n} \frac{\sigma_{u u} \sigma_{e e} \sum_{t=1}^{n}\left(r_{t} / s_{t}\right)}{\left(\sum_{t=1}^{n} r_{t}\left(x_{t}-\bar{x}\right)^{2}\right)^{2}} \\
& +\frac{\beta^{2}\left\{Q_{1}\left(r_{H}, R\right) \sigma_{u u}^{2}+Q_{2}\left(r_{H}, R\right)\left(3 \sigma_{u u}^{2}\right)\right\}(n-1)^{2}}{\left(\sum_{t=1}^{n} r_{t}\left(x_{t}-\bar{x}\right)^{2}\right)^{2}} \\
A V A R\left(\hat{\beta}_{D G 2}\right) & =\frac{\beta^{2} \sigma_{u u} \sum_{t=1}^{n}\left(s_{t}^{2} / s r_{t}\right)\left(x_{t}-\bar{x}\right)^{2}}{\left(\sum_{t=1}^{n} s_{t}\left(x_{t}-\bar{x}\right)^{2}\right)^{2}}+\frac{\sigma_{e e}}{\sum_{t=1}^{n} s_{t}\left(x_{t}-\bar{x}\right)^{2}} \\
& +\frac{n-1}{n} \frac{\sigma_{u u} \sigma_{e e} \sum_{t=1}^{n}\left(s_{t} / r_{t}\right)}{\left(\sum_{t=1}^{n} s_{t}\left(x_{t}-\bar{x}\right)^{2}\right)^{2}} \\
& +\frac{\left\{Q_{1}\left(s_{H}, S\right) \sigma_{e e}^{2}+Q_{2}\left(s_{H}, s\right)\left(3 \sigma_{e e}^{2}\right)\right\}(n-1)^{2}}{\beta^{2}\left(\sum_{t=1}^{n} s_{t}\left(x_{t}-\bar{x}\right)^{2}\right)^{2}},
\end{aligned}
$$

onde

$$
\begin{gathered}
r_{H}^{-1}=n^{-1} \sum_{t=1}^{n} r_{t}^{-1}, \quad s_{H}^{-1}=n^{-1} \sum_{t=1}^{n} s_{t}^{-1} \\
Q_{1}\left(r_{H}, R\right)=\frac{1}{(n-1)^{2}}\left(2 n-2-\frac{3 n}{r_{H}}+\frac{6 n}{R}-\frac{3}{R}\right)+\frac{1}{(R-n)^{2}}\left(4 n-R-\frac{3 n}{r_{H}}\right) \\
+\frac{6}{R-n}-\frac{6 n}{(R-n)(n-1)}\left(\frac{1}{r_{H}}-\frac{1}{R}\right) \\
Q_{2}\left(r_{H}, R\right)= \\
\quad+\frac{1}{(n-1)^{2}}\left(\frac{n}{r_{H}}-\frac{2 n}{R}+\frac{3}{R}\right)+\frac{1}{(R-n)^{2}}\left(\frac{n}{r_{H}}+R-2 n\right) \\
+(R-n)(n-1)
\end{gathered}
$$


com definições correspondentes para $Q_{1}\left(s_{H}, S\right)$ e $Q_{2}\left(s_{H}, S\right)$.

Os valores dos parâmetros utilizados foram $\alpha=10 ; \beta=0,5 ; \quad \sigma_{e e}=50$ e vários valores para $\sigma_{u u}$ que serão especificados nas tabelas apresentadas adiante. Para gerações de valores de $x_{t}, t=1, \ldots, n$, consideramos $x_{t} \sim N\left(\mu_{x}, \sigma_{x x}\right)$ com $\mu_{x}=30 \mathrm{e}$ $\sigma_{x x}=64$.

\section{Duplicações não-balanceadas da variável explanatória.}

Das definições de $Q_{1}\left(s_{H}, S\right)$ e $Q_{2}\left(s_{H}, S\right)$ e de $A V A R\left(\hat{\beta}_{D G 2}\right)$ dadas em (4.115) verificamos que não podemos utilizar o estimador $\hat{\beta}_{D G 2}$ para comparações, pois este estimador exige que $s_{t}>1$ para pelo menos um $t, t=1, \ldots, n$, ou seja, $S-n>0$.

A seguir apresentamos os resultados numéricos obtidos ao comparar o EMV em relação ao estimador $\hat{\beta}_{D G 1}$ dado em (4.113), considerando a seguinte notação para a eficência assintótica relativa:

$$
\operatorname{EAR}\left(\widehat{\beta}_{M V} \mid \widehat{\beta}_{D G 1}\right)=\frac{A V A R\left(\widehat{\beta}_{D G 1}\right)}{A V A R\left(\widehat{\beta}_{M V}\right)} .
$$

Tabela 4.1 : Eficiência Assintótica Relativa, considerando $\mathrm{n}=30$ e $r=25 \%$

(Ou seja, 25\% das 30 observações da variável explanatória duplicadas)

\begin{tabular}{|c|c|r|r|r|r|r|c|}
\hline Valores de $\sigma_{u u}$ & 4 & 9 & 16 & 60 & 100 & 144 & $240^{*}$ \\
\hline \hline$A V A R\left(\widehat{\beta}_{M V}\right)$ & 0,0219 & 0,0580 & 0,0489 & 0,0299 & 0,0171 & 0,0106 & 0,0085 \\
\hline$A V A R\left(\widehat{\beta}_{D G 1}\right)$ & 0,0244 & 0,0674 & 0,0628 & 0,1638 & 0,3511 & 0,3778 & 2,5722 \\
\hline $\operatorname{EAR}\left(\widehat{\beta}_{M V}, \widehat{\beta}_{D G 1}\right)$ & 1,1128 & 1,1620 & 1,2847 & 5,4762 & 20,494 & 35,539 & 301,836 \\
\hline
\end{tabular}

(Observação: O sinal (*) indica que os valores simulados não satisfazem as condições suficientes estabelecidas no Apêndice $D$ para garantir que a matriz $E\left[\mathbf{A}_{n}\left(\theta^{(1)}\right) \mid \theta^{(0)}, x_{t}^{0}\right]$ seja negativa definida.) 
Tabela 4.2: Eficiência Assintótica Relativa, considerando $\mathrm{n}=60$ e $r=25 \%$.

\begin{tabular}{|c|c|c|r|r|r|r|c|}
\hline Valores de $\sigma_{u u}$ & 4 & 9 & 16 & 60 & 100 & 144 & $240^{*}$ \\
\hline \hline$A V A R\left(\widehat{\beta}_{M V}\right)$ & 0,0167 & 0,0301 & 0,0225 & 0,0132 & 0,0083 & 0,0056 & 0,0042 \\
\hline$A V A R\left(\widehat{\beta}_{D G 1}\right)$ & 0,0184 & 0,0348 & 0,0275 & 0,0531 & 0,1465 & 0,2862 & 0,7337 \\
\hline $\operatorname{EAR}\left(\widehat{\beta}_{M V}, \widehat{\beta}_{D G 1}\right)$ & 1,0998 & 1,1555 & 1,2240 & 4,0196 & 17,649 & 51,144 & 174,21 \\
\hline
\end{tabular}

Tabela 4.3: Eficiência Assintótica Relativa, considerando $\mathrm{n}=100$ e $r=25 \%$.

\begin{tabular}{|c|c|c|r|r|r|r|c|}
\hline Valores de $\sigma_{u u}$ & 4 & 9 & 16 & 60 & 100 & 144 & $240^{*}$ \\
\hline \hline$A V A R\left(\widehat{\beta}_{M V}\right)$ & 0,0085 & 0,0098 & 0,0096 & 0,0080 & 0,0048 & 0,0033 & 0,0025 \\
\hline$A V A R\left(\widehat{\beta}_{D G 1}\right)$ & 0,0095 & 0,0112 & 0,0118 & 0,0345 & 0,0642 & 0,1584 & 0,4146 \\
\hline $\operatorname{EAR}\left(\widehat{\beta}_{M V}, \widehat{\beta}_{D G 1}\right)$ & 1,1112 & 1,1359 & 1,2261 & 4,3128 & 13,442 & 47,585 & $165,56$. \\
\hline
\end{tabular}

Tabela 4.4: Eficiência Assintótica Relativa, considerando $\mathrm{n}=30$ e $r=50 \%$.

\begin{tabular}{|c|c|c|r|r|r|r|c|}
\hline Valores de $\sigma_{u u}$ & 4 & 9 & 16 & 60 & 100 & 144 & $240^{*}$ \\
\hline \hline$A V A R\left(\widehat{\beta}_{M V}\right)$ & 0,0230 & 0,0693 & 0,0555 & 0,1433 & 0,0343 & 0,0171 & 0,0104 \\
\hline$A V A R\left(\widehat{\beta}_{D G 1}\right)$ & 0,0242 & 0,0507 & 0,0306 & 0,1187 & 0,0726 & 0,2264 & 1,3867 \\
\hline $\operatorname{EAR}\left(\widehat{\beta}_{M V}, \widehat{\beta}_{D G 1}\right)$ & 1,0543 & 0,7321 & 0,5508 & 0,8287 & 6,6403 & 13,244 & 132,926 \\
\hline
\end{tabular}

Tabela 4.5: Eficiência Assintótica Relativa, considerando $\mathrm{n}=60$ e $r=50 \%$.

\begin{tabular}{|c|c|c|r|r|r|r|c|}
\hline Valores de $\sigma_{u u}$ & 4 & 9 & 16 & 60 & 100 & 144 & $240^{*}$ \\
\hline \hline$A V A R\left(\widehat{\beta}_{M V}\right)$ & 0,0149 & 0,0351 & 0,0320 & 0,0852 & 0,0173 & 0,0086 & 0,0051 \\
\hline$A V A R\left(\widehat{\beta}_{D G 1}\right)$ & 0,0157 & 0,0256 & 0,0165 & 0,0425 & 0,0798 & 0,1520 & 0,3917 \\
$\operatorname{EAR}\left(\widehat{\beta}_{M V}, \widehat{\beta}_{D G 1}\right)$ & 1,055 & 0,7303 & 0,5157 & 0,4985 & 4,6192 & 17,669 & 77,179 \\
\hline
\end{tabular}


Tabela 4.6: Eficiência Assintótica Relativa, considerando $n=100$ e $r=50 \%$.

\begin{tabular}{|c|c|r|r|r|r|r|c|}
\hline Valores de $\sigma_{u u}$ & 4 & 9 & 16 & 60 & 100 & 144 & $240^{*}$ \\
\hline \hline$A V A R\left(\widehat{\beta}_{M V}\right)$ & 0,0090 & 0,0135 & 0,0228 & 0,0510 & 0,0104 & 0,0051 & 0,0030 \\
\hline$A V A R\left(\widehat{\beta}_{D G 1}\right)$ & 0,0095 & 0,0105 & 0,0110 & 0,0249 & 0,0397 & 0,0832 & 0,1945 \\
\hline $\operatorname{EAR}\left(\widehat{\beta}_{M V}, \widehat{\beta}_{D G 1}\right)$ & 1,0462 & 0,7820 & 0,4836 & 0,4884 & 3,8083 & 16,186 & 64,839 \\
\hline
\end{tabular}

Tabela 4.7: Eficiência Assintótica Relativa, considerando $\mathrm{n}=30$ e $r=60 \%$.

\begin{tabular}{|c|c|c|r|r|r|r|c|}
\hline Valores de $\sigma_{u u}$ & 4 & 9 & 16 & $60^{*}$ & 100 & 144 & $240^{*}$ \\
\hline \hline$A V A R\left(\widehat{\beta}_{M V}\right)$ & 0,0221 & 0,0684 & 0,0340 & 1,112 & 0,0614 & 0,0240 & 0,0116 \\
\hline$A V A R\left(\widehat{\beta}_{D G 1}\right)$ & 0,0238 & 0,0670 & 0,0303 & 0,1044 & 0,2121 & 0,2025 & 1,0383 \\
\hline $\operatorname{EAR}\left(\widehat{\beta}_{M V}, \widehat{\beta}_{D G 1}\right)$ & 1,0737 & 0,9801 & 0,8915 & 0,0939 & 3,4561 & 8,4413 & 89,359 \\
\hline
\end{tabular}

Tabela 4.8: Eficiência Assintótica Relativa, considerando $\mathrm{n}=60$ e $r=60 \%$.

\begin{tabular}{|c|c|c|c|c|r|r|c|}
\hline Valores de $\sigma_{u u}$ & 4 & 9 & 16 & $60^{*}$ & 100 & 144 & $240^{*}$ \\
\hline \hline$A V A R\left(\widehat{\beta}_{M V}\right)$ & 0,0142 & 0,0263 & 0,0190 & 2,1070 & 0,0330 & 0,0122 & 0,0057 \\
\hline$A V A R\left(\widehat{\beta}_{D G 1}\right)$ & 0,0155 & 0,0247 & 0,0163 & 0,0380 & 0,0713 & 0,0561 & 0,3323 \\
\hline $\operatorname{EAR}\left(\widehat{\beta}_{M V}, \widehat{\beta}_{D G 1}\right)$ & 1,0899 & 0,9428 & 0,8609 & 0,0180 & 2,1569 & 4,6109 & 58,578 \\
\hline
\end{tabular}

Tabela 4.9: Eficiência Assintótica Relativa, considerando $\mathrm{n}=100$ e $r=60 \%$.

\begin{tabular}{|c|c|c|c|c|c|c|c|}
\hline Valores de $\sigma_{u u}$ & 4 & 9 & 16 & $60^{*}$ & 100 & 144 & $240^{*}$ \\
\hline \hline$A V A R\left(\widehat{\beta}_{M V}\right)$ & 0,0086 & 0,0108 & 0,0129 & 1,188 & 0,0212 & 0,0072 & 0,0034 \\
\hline$A V A R\left(\widehat{\beta}_{D G 1}\right)$ & 0,0094 & 0,0103 & 0,0106 & 0,0229 & 0,0347 & 0,0731 & 0,1686 \\
\hline $\operatorname{EAR}\left(\widehat{\beta}_{M V}, \widehat{\beta}_{D G 1}\right)$ & 1,0903 & 0,9572 & 0,8240 & 0,0193 & 1,6416 & 10,223 & 48,552 \\
\hline
\end{tabular}


Tabela 4.10: Eficiência Assintótica Relativa, considerando $\mathrm{n}=30$ e $r=80 \%$.

\begin{tabular}{|l|c|c|c|c|c|c|l|}
\hline Valores de $\sigma_{u u}$ & 4 & 9 & 16 & $60^{*}$ & 100 & 144 & $240^{*}$ \\
\hline \hline$A V A R\left(\widehat{\beta}_{M V}\right)$ & 0,0284 & 0,0468 & 0,0286 & 0,2495 & 0,3270 & 0,0576 & 0,0153 \\
\hline$A V A R\left(\widehat{\beta}_{D G 1}\right)$ & 0,0307 & 0,0477 & 0,0297 & 0,0568 & 0,1796 & 0,1720 & 0,7849 \\
\hline $\operatorname{EAR}\left(\widehat{\beta}_{M V}, \widehat{\beta}_{D G 1}\right)$ & 1,0817 & 1,019 & 1,0378 & 0,2276 & 0,5491 & 2,9890 & 51,451 \\
\hline
\end{tabular}

Tabela 4.11: Eficiência Assintótica Relativa, considerando $n=60$ e $r=80 \%$.

\begin{tabular}{|c|c|c|c|c|r|r|c|}
\hline Valores de $\sigma_{u u}$ & 4 & 9 & 16 & $60^{*}$ & $100^{*}$ & 144 & $240^{*}$ \\
\hline \hline$A V A R\left(\widehat{\beta}_{M V}\right)$ & 0,0166 & 0,0311 & 0,0157 & 0,1207 & 0,2126 & 0,0283 & 0,0075 \\
\hline$A V A R\left(\widehat{\beta}_{D G 1}\right)$ & 0,0179 & 0,0320 & 0,0156 & 0,0285 & 0,0654 & 0,0946 & 0,2590 \\
\hline $\operatorname{EAR}\left(\widehat{\beta}_{M V}, \widehat{\beta}_{D G 1}\right)$ & 1,0823 & 1,0280 & 0,9919 & 0,2366 & 0,3077 & 3,3482 & 34,392 \\
\hline
\end{tabular}

Tabela 4.12: Eficiência Assintótica Relativa, considerando $\mathrm{n}=100$ e $r=80 \%$.

\begin{tabular}{|c|r|r|r|r|r|r|c|}
\hline Valores de $\sigma_{u u}$ & 4 & 9 & 16 & $60^{*}$ & $100^{*}$ & 144 & $240^{*}$ \\
\hline \hline$A V A R\left(\widehat{\beta}_{M V}\right)$ & 0,0101 & 0,0148 & 0,0105 & 0,0877 & 0,2241 & 0,0173 & 0,0045 \\
\hline$A V A R\left(\widehat{\beta}_{D G 1}\right)$ & 0,0107 & 0,0147 & 0,0104 & 0,0188 & 0,0298 & 0,0549 & 0,1260 \\
\hline $\operatorname{EAR}\left(\widehat{\beta}_{M V}, \widehat{\beta}_{D G 1}\right)$ & 1,0647 & 0,9968 & 0,9831 & 0,2141 & 0,1328 & 3,1748 & 28,114 \\
\hline
\end{tabular}

Tabela 4.13: Eficiência Assintótica Relativa, considerando $\mathrm{n}=30$ e $r=100 \%$.

\begin{tabular}{|c|c|c|r|r|r|r|c|}
\hline Valores de $\sigma_{u u}$ & 4 & 9 & 16 & 60 & $100^{*}$ & 144 & $240^{*}$ \\
\hline \hline$A V A R\left(\widehat{\beta}_{M V}\right)$ & 0,0216 & 0,0450 & 0,0267 & 0,1033 & 143,7 & 0,1895 & 0,0214 \\
\hline$A V A R\left(\widehat{\beta}_{D G 1}\right)$ & 0,0216 & 0,0441 & 0,0258 & 0,0536 & 0,1291 & 0,1587 & 0,6274 \\
\hline $\operatorname{EAR}\left(\widehat{\beta}_{M V}, \widehat{\beta}_{D G 1}\right)$ & 0,9977 & 0,9816 & 0,9684 & 0,5187 & 0,0009 & 0,8376 & 29,344 \\
\hline
\end{tabular}


Tabela 4.14: Eficiência Assintótica Relativa, considerando $\mathrm{n}=60$ e $r=100 \%$.

\begin{tabular}{|c|c|c|c|c|c|c|c|}
\hline Valores de $\sigma_{u u}$ & 4 & 9 & 16 & 60 & $100^{*}$ & 144 & 240 \\
\hline \hline$A V A R\left(\widehat{\beta}_{M V}\right)$ & 0,0138 & 0,0295 & 0,0147 & 0,0502 & 8,113 & 0,0908 & 0,0108 \\
\hline$A V A R\left(\widehat{\beta}_{D G 1}\right)$ & 0,0137 & 0,0289 & 0,0142 & 0,0265 & 0,0528 & 0,0843 & 0,2162 \\
\hline $\operatorname{EAR}\left(\widehat{\beta}_{M V}, \widehat{\beta}_{D G 1}\right)$ & 0,9975 & 0,9783 & 0,9673 & 0,5272 & 0,0065 & 0,9293 & 20,029 \\
\hline
\end{tabular}

Tabela 4.15: Eficiência Assintótica Relativa, considerando $\mathrm{n}=100$ e $r=100 \%$.

\begin{tabular}{|c|c|c|c|c|c|c|c|}
\hline Valores de $\sigma_{u u}$ & 4 & 9 & 16 & $60^{*}$ & $100^{*}$ & $144^{*}$ & 240 \\
\hline \hline$A V A R\left(\widehat{\beta}_{M V}\right)$ & 0,00993 & 0,0096 & 0,0098 & 0,0363 & 0,9490 & 0,1407 & 0,0066 \\
\hline$A V A R\left(\widehat{\beta}_{D G 1}\right)$ & 0,00990 & 0,0095 & 0,0094 & 0,0180 & 0,0265 & 0,0259 & 0,0863 \\
\hline $\operatorname{EAR}\left(\widehat{\beta}_{M V}, \widehat{\beta}_{D G 1}\right)$ & 0,9972 & 0,9878 & 0,9649 & 0,4959 & 0,0279 & 0,1843 & 13,161 \\
\hline
\end{tabular}

\section{Avaliação dos resultados}

Pelas tabelas apresentadas verificamos que o estimador de MV é mais eficiente em relação ao estimador alternativo quando a porcentagem das replicações sobre a variável explanatória é baixa ( $\mathrm{r}=25 \%$ das observações). $\mathrm{O}$ aumento desta porcentagem, a diminuição de $\sigma_{u u}$ e o aumento do tamanho da amostra favorecem ambos os estimadores, mas preponderantemente o estimador alternativo $\hat{\beta}_{D G 1}$ dado em (4.113). Descartando os resultados nos quais as condições dadas no Apêndice D não são satisfeitas, em geral, o estimador de MV é mais eficiente que o alternativo em questão. Para o caso balanceado em que $s_{t} / r_{t}=k, t=1, \ldots, n$, os resultados obtidos são muito semelhantes ao caso anterior quando se considera o primeiro estimador alternativo, e em relação ao segundo estimador, o EMV, em geral, foi mais eficiente, como podemos observar nas duas próximas tabelas. 
Tabela 4.16 : EAR, considerando $\mathrm{n}=30, r=25 \%$ e $s_{t} / r_{t}=1$.

\begin{tabular}{|c|c|c|r|r|r|r|c|}
\hline Valores de $\sigma_{u u}$ & 4 & 9 & 16 & 60 & 100 & 144 & 240 \\
\hline \hline$A V A R\left(\widehat{\beta}_{M V}\right)$ & 0,0179 & 0,0475 & 0,0023 & 0,0219 & 0,0161 & 0,0111 & 0,0088 \\
\hline$A V A R\left(\widehat{\beta}_{D G 1}\right)$ & 0,0181 & 0,0051 & 0,0273 & 0,0878 & 0,2991 & 0,4589 & 1,4415 \\
\hline$A V A R\left(\widehat{\beta}_{D G 2}\right)$ & 0,3031 & 1,6693 & 0,4454 & 0,6442 & 1,1728 & 1,0074 & 1,3455 \\
\hline $\operatorname{EAR}\left(\widehat{\beta}_{M V}, \widehat{\beta}_{D G 1}\right)$ & 1,0065 & 1,0851 & 1,1399 & 4,0096 & 18,523 & 41,186 & 162,66 \\
\hline $\operatorname{EAR}\left(\widehat{\beta}_{M V}, \widehat{\beta}_{D G 2}\right)$ & 1,0903 & 35,074 & 18,594 & 29,411 & 72,618 & 90,403 & 151,84 \\
\hline
\end{tabular}

Tabela 4.17: EAR, considerando $\mathrm{n}=100, r=80 \%$ e $s_{t} / r_{t}=1$.

\begin{tabular}{|c|c|c|r|r|r|r|c|}
\hline Valores de $\sigma_{u u}$ & 4 & 9 & 16 & 60 & 100 & 144 & 240 \\
\hline \hline$A V A R\left(\widehat{\beta}_{M V}\right)$ & 0,0048 & 0,0075 & 0,0053 & 0,0083 & 0,0113 & 0,0141 & 0,1545 \\
\hline$A V A R\left(\widehat{\beta}_{D G 1}\right)$ & 0,0048 & 0,0074 & 0,0053 & 0,0076 & 0,0091 & 0,0096 & 0247 \\
\hline$A V A R\left(\widehat{\beta}_{D G 2}\right)$ & 0,0434 & 0,0021 & 0,0464 & 0,0563 & 0,0532 & 0,0424 & 0,0804 \\
\hline $\operatorname{EAR}\left(\widehat{\beta}_{M V}, \widehat{\beta}_{D G 1}\right)$ & 0,9995 & 0,9972 & 0,9943 & 0,9224 & 0,8072 & 0,6806 & 0,1597 \\
\hline $\operatorname{EAR}\left(\widehat{\beta}_{M V}, \widehat{\beta}_{D G 2}\right)$ & 9,0259 & 12,348 & 8,6793 & 6,7934 & 4,6948 & 3,0058 & 0,5206 \\
\hline
\end{tabular}




\section{Capítulo 5}

\section{Contribuições, Conclusão e Estudos Futuros da Tese}

\subsection{Contribuições}

Esta tese pretendeu contribuir, dentro das limitações especificadas ad-hoc, nos seguintes pontos:

No Capítulo 2, no Modelo Estrutural Normal, as principais contribuições são:

1. - Caso balanceado, com $r_{t}=r$ e $s_{t}=1$, isto é, replicação só da variável explanatória. Foi obtido um polinômio de terceiro grau em $\beta$, redutível ao segundo, como produto de polinômios de primeiro e segundo grau, gerando três raízes reais:

$$
\widehat{\beta}_{1}^{\prime}=\frac{(r-1) S_{X Y}}{r S_{x x}-t_{X X}} \quad \text { e } \quad \widehat{\beta}_{1}^{\prime \prime}=\widehat{\beta}_{1}^{\prime \prime \prime}=\frac{S_{Y Y}}{S_{X Y}} .
$$

A primeira raíz pode ser tomada como estimador de máxima verossilhança para $\beta$, pois as outras (duas raízes iguais) caem fora do espaço paramétrico admitido. Sob as condições de regularidade usuais (veja, por exemplo, Sen e Singer, 1993), são investigados as propriedades assintóticas deste estimador, e obtida a matriz de covariâncias assintóticas dos EMVs resultantes. Este modelo não se reduz a um caso particular considerado em Chan e Mak, (1979), pois não é possível fazer $s_{t}=r=1$. 
2. - Caso não-balanceado, com $r_{t} \geq 1, s_{t}=1 ; t=1, \ldots, n$, replicação só da variável explanatória. Neste caso não há uma solução analítica fechada, pois as equações de estimação são irredutíveis a uma equação em $\beta$; para mais detalhes veja, entre outros, Miller et alli (1977); porém é possível obter a matriz de covariância assintótica dos EMVs. Aqui, há uma solução iterativa via algorítmo EM (ver Schaffer e Purdy, 1996).

3. - Caso balanceado com $r_{t}=r, s_{t}=s$. Obtem-se um polinômio de quarto grau em $\beta$; sendo os casos - Chan e Mak (1979) e o do ítem-(1) - particulares deste.

4. - Caso não-balanceado, com $r_{t} / s_{t}=k, t=1, \ldots, n$. Não há solução analítica.

No Capítulo 3 , com o Modelo Estrutural Normal, as contribuições mais relevantes são: (1) a obtenção da distribuição assintótica de $\widehat{\beta}_{N B 3}$ pelo método Delta, que exigiu o uso do Teorema Central de Limite de Liapounov e o Teorema que denominamos GleserRupert, considerado em Gallo (1982).

(2) a obtenção dos estimadores alternativos corrigidos e com pesos melhorados, seguindo as sugestões em Fuller (1987) e, Purdy e Scharff (1996).

No Capítulo 4 a técnica de replicação desenvolvida em Chan e Mak (1979) e o conjunto de condições de regularidade estabelecido em Mak (1982) são aplicados ao Modelo Linear Funcional Normal para obter os estimadores de máxima verossimilhança de $\theta$, sem nenhuma restrição paramétrica. No caso não-balanceado, mas com $s_{t} / r_{t}=k$, obtemos um polinômio de quarto grau em $\beta$. Com probabilidade tendendo a um, este polinômio tem pelo menos uma raíz real, quando $r$ tende ao infinito, conforme os resultados em Chan e Mak (1979). Com as condições de regularidade e algumas suposições adicionais provamos que uma destas raízes reais é um EMV de $\beta$. Obtivemos a matriz de covariâncias dos EMVs a partir de uma fórmula dada em Mak (1982).

\subsection{Conclusão e Estudos Futuros.}

Em grande parte deste trabalho discutimos a possibilidade de se considerar a técnica de replicações de variáveis, como um método de obtenção dos EMVs, na ausência de suposições restritivas sobre os parâmetros dos modelos funcional e estrutural, suposições 
estas utilizadas para, respectivamente, obtenção da estimação de máxima verossimilhança dos parâmetros estruturais e identificação do modelo estrutural. As conclusões finais mais relevantes são resumidas a seguir.

- Em todas as simulações feitas obtivemos pelo menos duas raízes reais para o polinômio de quarto grau obtido a partir do modelo funcional normal com replicação não balanceada, quando $\frac{s_{t}}{r_{t}}=k$. Aplicamos, ainda, um algoritmo tipo EM (Kimura, 1992) para obtençâo dos EMVs; os dois processos levam a uma mesma estimativa do parâmetro. No entanto, verificamos nas simulações que, em alguns casos, este algoritmo depende muito dos valores iniciais arbitrados quando o tamanho da amostra é pequeno, casos, estes, que resultaram em estimativas que não maximizaram a função log-verossimilhança do modelo.

- Em todos os casos investigados, no Modelo Funcional, a estimação do vetor de parâmetros incidentais $\left(\mathrm{x}=\left(x_{1} \ldots \ldots x_{n}\right)\right)$ é efetuada através do enfoque de Máxima Verossimilhança usual, considerando os parâmetros estruturais fixos; este processo é justificado em Richard (1961).

- Através de estudos de simulações os EMVs obtidos analiticamente, via replicações no Modelo Normal Estrutural, são comparados com os estimadores consistentes "melhorados"obtidos no Capítulo 3 . Os resultados estão no final do Capítulo 4.

- No Modelo Funcional Normal os EMVs obtidos via replicações de variáveis foram comparados com os estimadores propostos em Dorf e Gurland (1960); os resultados são apresentados nas Tabelas de 4.1 a 4.15 , no final do Capítulo 4.

Todas as condições de identificabilidade são consideradas para obter os estimadores alternativos (no Cap.3) no caso de replicação balanceada. São obtidos estimadores de máxima verossimilhança dos parâmetros: (a) $\sigma_{u u}$, para a condição S-1 de identificabilidade, usando uma amostra só de $\mathrm{X}_{t}$; (b) $\lambda=\sigma_{e e} / \sigma_{u u}$, para a condição S -2 de identificabilidade; (c) $k_{x x}=\frac{\sigma_{x x}}{\sigma_{x x}+\sigma_{u u}}$, fator de atenuação. No caso de replicações não- 
balanceadas com $r_{t} \geq 1$, replicação só da variável explanatória, trabalhamos sempre a partir do estimador de máxima verossimilhança de $\beta$ obtido sob a suposição (S-1). O estimador da matriz de covariâncias dos EMVs, é obtido em todos os casos relevantes investigados.

- No Modelo Normal Estrutural, soluções explícitas são obtidas no caso de replicação balanceada só da variável explanatória. Os estimadores são comparados com estimadores obtidos via abordagem do método dos momentos; os quais equivalem aos EMVs obtidos sob as suposições adicionais identificadoras no modelo Normal Estrutural. Finalmente, alguns estudos de simulação ilustram o comportamento dos estimadores para vários tamanhos da amostra, sob diferentes valores da variância dos erros.

- O Modelo Normal Estrutural só admite solução analítica quando as replicações são balanceadas. Já o Modelo Normal Funcional admite solução para replicações nãobalanceadas, mas com restrição $\left(\frac{r_{t}}{s_{t}}=k\right)$ em relação ao número de replicações. Nos casos sem solução analítica a única alternativa é uma solução via Algorítmo EM no Modelo Normal Estrutural, e algorítmo tipo EM no Funcional.

-O caso mais geral, para que as equações de estimação de verossimilhança sejam redutíveis a uma equação polinomial em função de $\beta$, ou seja, que tenha solução analítica, é o caso $r_{t}=r$ e $s_{t}=s$. O caso $r_{t} / s_{t}=k$ (constante) foi tentado, sem sucesso.

- Finalmente, nos Apêndices, resumimos alguns resultados clássicos da literatura, utilizados com certa freqüência, no decorrer da tese, e também resultados recentes usados para garantir a convergência no uso do método Delta (Sen e Singer, 1993) e as condições imposta em Mak (1982) para garantir os resultados assintóticos dos EMVs obtidos. Além disso, detalhamos o desenvolvimentos de alguns cálculos referentes às demonstrações de teoremas e a outros resultados. As extensões, continuidades deste trabalho e algumas sugestões são apresentados a seguir. 


\section{Sugestões para pesquisa futura}

O modelo funcional normal bivariado é um caso particular do modelo calibração comparativa quando o número de instrumento é igual a dois (ver, por exemplo, Rojas, 1995). Num estudo futuro poderíamos estender este caso para o modelo funcional normal multivariado que equivale ao modelo de calibração comparativa para $\mathrm{p}$ instrumentos. Podemos também considerar uma família mais geral para a distribuição de erros de mensurações que a normal, por exemplo a família elíptica, que garanta uma inferência estatística mais robusta, isto é, menos vulnerável a "outliers", como seria o caso da distribuição t-student, que é mais "resistente "à presença de observações extremas (ver, por exemplo, Vilca Labra, 1996). Vale a mesma proposta para o Modelo Normal Estrutural Bivariado. 


\section{Apêndice A}

\section{Implementação dos Algoritmos EM e do Tipo EM}

Neste apêndice demonstramos alguns resultados utilizados na implementação do algoritmo EM e do tipo EM, que aparecem nos artigos de Schaffer e Purdy (1996) e Kimura (1992), respectivamente.

\section{A.1 Cálculos para Implementação do Algoritmo EM (Scha- fer e Purdy, 1996)}

Suposições do Modelo: as mesmas adotadas para o Modelo Estrutural Normal dadas em (2.2), ou seja:

$$
\begin{aligned}
Y_{t} \mid X_{t}, x_{t} & \sim N_{1}\left(\beta_{0}+\beta_{1} x_{t} ; \sigma_{e e}\right), \\
X_{t j} \mid x_{t} \sim N\left(x_{t}, \sigma_{u u}\right), \quad V\left(X_{t j} \mid x_{t}\right) & =V\left(x_{t} \mid x_{t}\right)+V\left(u_{t j} \mid x_{t}\right)=0+V\left(u_{t j}\right)=\sigma_{u u}, \\
x_{t} & \sim N\left(\mu_{x}, \sigma_{x x}\right),
\end{aligned}
$$

com

$$
Y_{t}=\beta_{0}+\beta_{1} x_{t}+e_{t}
$$




$$
X_{t j}=x_{t}+u_{t j} ;, \quad j=1, \ldots, r_{t}, \quad t=1, \ldots, n .
$$

\section{Mudança de Notações em Relação ao Artigo:}

Observe que a notação do lado direito das identidades abaixo é a adotada em Schafer e Purdy (1996).

$$
\sigma_{e e}=\sigma^{2} ; \sigma_{u u}=\sigma_{z}^{2} ; \sigma_{x x}=\sigma_{x}^{2} ; \mu_{x}=\mu ; X_{t j}=Z_{t j} ; Y_{t}=y_{t}
$$

Portanto, após algumas manipulações algébricas, obtemos

$$
\begin{aligned}
\operatorname{Var}\left(Y_{t}\right) & =V\left(\beta_{0}+\beta_{1} x_{t}+e_{t}\right)=\beta^{2} \sigma_{x x}+\sigma_{e e}, \\
\operatorname{Var}\left(X_{t j}\right) & =V\left(x_{t}+u_{t j}\right)=\sigma_{x x}+\sigma_{u u}, \\
\operatorname{Cov}\left(X_{t j}, X_{t k}\right) & =\sigma_{x x} \quad\left(j \neq k ; j, k=1,2, \cdots, r_{t}\right), \\
\operatorname{Cov}\left(x_{t}, Y_{t}\right) & =\beta_{1} \sigma_{x x}, \\
\operatorname{Cov}\left(x_{t}, X_{t j}\right) & =\sigma_{x x} \quad\left(\text { para } j=1,2, \cdots, r_{t}\right), \\
\operatorname{Cov}\left(Y_{t}, X_{t j}\right) & =\beta_{1} \sigma_{x x} .
\end{aligned}
$$

De (A.1) e das suposições do modelo temos (observações completas)

$$
\left(\begin{array}{c}
x_{t} \\
\mathbf{X}_{t} \\
Y_{t}
\end{array}\right) \sim N_{r_{t}+2}\left[\left(\begin{array}{c}
\mu_{x} \\
\mu_{x} \mathbf{1}_{r_{t} 1} \\
\beta_{0}+\beta_{1} \mu_{x}
\end{array}\right), \sum_{x_{t}, \mathbf{X}_{t}, Y_{t}}\right]
$$

onde

$$
\sum_{x_{t}, \mathbf{X}_{t}, Y_{t}}=\left(\begin{array}{ccc}
\sigma_{x x} & \sigma_{x x} \mathbf{1}_{1 r_{t}} & \beta_{1} \sigma_{x x} \\
\sigma_{x x} \mathbf{1}_{r_{t} 1} & \sigma_{x x} 1_{r_{t} r_{t}}+\sigma_{u u} \mathbf{I}_{\mathbf{r}_{t}} & \beta_{1} \sigma_{x x} \mathbf{1}_{r_{t} 1} \\
\beta_{1} \sigma_{x x} & \beta_{1} \sigma_{x x} \mathbf{1}_{1 r_{t}} & \beta_{1}^{2} \sigma_{x x}+\sigma_{e e}
\end{array}\right) .
$$

Consideremos as seguintes partições das matrizes acima

$$
\left(\begin{array}{c}
x_{t} \\
\mathrm{X}_{t} \\
Y_{t}
\end{array}\right)=\left(\begin{array}{c}
\mathrm{W}_{1} \\
\mathrm{~W}_{2}
\end{array}\right)
$$


onde

$$
\mathrm{W}_{1}=x_{t} \text { e } \mathrm{W}_{2}=\left(\begin{array}{c}
\mathrm{X}_{t} \\
Y_{t}
\end{array}\right)
$$

com

$$
E\left(\mathrm{~W}_{1}\right)=\mu_{x}, E\left(\mathrm{~W}_{2}\right)=\left(\begin{array}{c}
\mu_{x} 1_{r_{t} 1} \\
\beta_{0}+\beta_{1} \mu_{x}
\end{array}\right),
$$

e a partição da matriz variância-covariância,

$$
\Sigma_{x_{t}, \mathrm{X}_{t}, Y_{t}}=\left(\begin{array}{cc}
\Sigma_{11} & \Sigma_{12} \\
\Sigma_{21} & \Sigma_{22}
\end{array}\right),
$$

onde

$$
\begin{gathered}
\Sigma_{11}=\sigma_{x x}, \\
\Sigma_{12}=\left(\sigma_{x x} \mathbf{1}_{1 r_{t}}, \beta_{1} \sigma_{x x}\right), \\
\Sigma_{21}=\Sigma_{21}^{\prime}, \\
\Sigma_{22}=\left(\begin{array}{cc}
\sigma_{x x} 1_{r_{t} r_{t}}+\sigma_{u u} \mathbf{I}_{r_{t}} & \beta_{1} \sigma_{x x} 1_{r_{t} 1} \\
\beta_{1} \sigma_{x x} 1_{1 r_{t}} & \beta_{1}^{2} \sigma_{x x}+\sigma_{e e}
\end{array}\right) .
\end{gathered}
$$

Usando o resultado (4.6) em Johnson e Wichern (1992, pg. 138), temos

$$
\begin{gathered}
E\left(x_{t} \mid \mathrm{X}_{t}, Y_{t}\right)=\mu_{x}+\Sigma_{12} \Sigma_{22}^{-1}\left(\mathbf{W}_{2}-E\left(\mathbf{W}_{2}\right)\right), \\
\operatorname{Var}\left(x_{t} \mid \mathrm{X}_{t}, Y_{t}\right)=\Sigma_{11}-\Sigma_{12} \Sigma_{22}^{-1} \Sigma_{21} .
\end{gathered}
$$

Cálculo de $\operatorname{Var}\left(x_{t} \mid \mathrm{X}_{t}, Y_{t}\right)$

Do artigo de Chan e Mak (1979), temos

$$
\left(\Sigma_{22}\right)^{-1}=\left(\sigma_{u u}^{-1}\right)\left(\sigma_{e e}^{-1}\right)\left(a_{t}^{-1}\right)\left(\begin{array}{cc}
a_{t} \sigma_{e e} \mathbf{I}_{r_{t}}-\sigma_{u u}^{-1} \sigma_{e e} 1_{r_{t} r_{t}} & -\beta_{1} 1_{r_{t} 1} \\
-\beta_{1} 1_{1 r_{t}} & \sigma_{u u} a_{t}-\beta_{1}^{2} \sigma_{u u} \sigma_{e e}^{-1}
\end{array}\right) .
$$


Após algumas manipulações algébricas, obtemos

$$
\begin{aligned}
\Sigma_{12}\left(\Sigma_{22}\right)^{-1} & =K\left(\begin{array}{ll}
\sigma_{x x} \mathbf{1}_{1 r_{t}} & \beta_{1} \sigma_{x x}
\end{array}\right)\left(\begin{array}{cc}
a_{t} \sigma_{e e} \mathbf{I}_{r_{t}}-\sigma_{u u}^{-1} \sigma_{e e} \mathbf{1}_{r_{t} r_{t}} & -\beta_{1} \mathbf{1}_{r_{t} 1} \\
-\beta_{1} \mathbf{1}_{1 r_{t}} & \sigma_{u u}\left(a_{t}-\beta_{1}^{2} \sigma_{e e}^{-1}\right)
\end{array}\right) \\
& =K\left(\sigma_{e e} \mathbf{1}_{1 r_{t}} ; \beta_{1} \sigma_{u u}\right),
\end{aligned}
$$

e

$$
\begin{aligned}
\Sigma_{12} \Sigma_{22}^{-1} \Sigma_{21} & =K\left(\begin{array}{ll}
\sigma_{e e} \mathbf{1}_{1 r_{t}} & \beta_{1} \sigma_{u u}
\end{array}\right)\left(\begin{array}{c}
\sigma_{x x} \mathbf{1}_{1 r_{t}} \\
\beta_{1} \sigma_{x x}
\end{array}\right) \\
& =a_{t}^{-1}\left(\sigma_{x x}\right)\left(r_{t} \sigma_{u u}^{-1}+\beta_{1}^{2} \sigma_{e e}^{-1}\right),
\end{aligned}
$$

em que $\mathrm{K}=\left(\sigma_{u u}^{-1}\right)\left(\sigma_{e e}^{-1}\right)\left(a_{t}^{-1}\right) \quad$ e $\quad a_{t}=\sigma_{x x}^{-1}+r_{t} \sigma_{u u}^{-1}+\beta_{1}^{2} \sigma_{e e}^{-1}$.

Substituindo $(A .3)$ e $(A .4)$ em $(A .2)$ temos

$$
\begin{aligned}
\operatorname{Var}\left(x_{t} \mid \mathbf{X}_{t}, Y_{t}\right) & =\sigma_{x x}-a_{t}^{-1} \sigma_{x x}\left(r_{t} \sigma_{u u}^{-1}+\beta_{1}^{2} \sigma_{e e}^{-1}\right) \\
& =\frac{\sigma_{e e} \sigma_{x x} \sigma_{u u}}{\sigma_{u u}\left(\sigma_{e e}+\beta_{1}^{2} \sigma_{x x}\right)+r_{t} \sigma_{x x} \sigma_{e e}}
\end{aligned}
$$

\section{A.2 Cálculos para Implementação do Algoritmo Tipo EM (Kimura, 1992)}

Este algoritmo foi desenvolvido em Kimura (1992), para obtenção dos EMV'S dos parâmetros estruturais de um Modelo Funcional Linear. Aqui, consideramos as replicações das variáveis explanatória e resposta.

Iteração Zero. Valores iniciais:

$\sigma_{u u}^{(0)}=\sigma_{e e}^{(0)}=1, \beta^{(0)}=1, \alpha^{(0)}=0$

Passo 1: Estimar $x_{t} ; g_{t}=\hat{x}_{t}$, onde 


$$
\hat{x}_{t}=g_{t}=\frac{\sigma_{u u}^{-1} r_{t} \bar{X}_{t .}+s_{t} \beta \sigma_{e e}^{-1}\left(\bar{Y}_{t .}-\alpha\right)}{r_{t} \sigma_{u u}^{-1}+s_{t} \beta^{2} \sigma_{e e}^{-1}} .
$$

Passo 2: Maximizar a log-verossimilhança $\hat{l}$, dada em (4.23).

A maximização de $\hat{l}$ será feita em relação aos parâmetros estruturais. Reescrevemos, a seguir, a função $\hat{l}$ :

$$
\begin{aligned}
\hat{l}= & -\frac{n}{2}(\bar{r}+\bar{s}) \log (2 \pi)-\frac{1}{2} n \bar{r} \log \sigma_{u u}-\frac{1}{2} n \bar{s} \log \sigma_{e e} \\
& -\frac{1}{2}\left[\sigma_{u u}^{-1} \sum_{t=1}^{n} \sum_{j=1}^{r_{t}}\left(X_{t j}-\hat{x}_{t}\right)^{2}+\sigma_{e e}^{-1} \sum_{t=1}^{n} \sum_{l=1}^{s_{t}}\left(Y_{t l}-\alpha-\beta \hat{x}_{t}\right)^{2}\right] .
\end{aligned}
$$

As equações de estimação de máxima verossimilhança para $\alpha$ e $\beta$ serão

$$
\begin{aligned}
\frac{\partial \hat{l}}{\partial \alpha} & =0 \Longrightarrow-\frac{1}{2} \sigma_{e e}^{-1} \sum_{t=1}^{n} \sum_{l=1}^{s_{t}} \frac{\partial\left(Y_{t l}-\alpha-\beta \hat{x}_{t}\right)^{2}}{\partial \alpha}=0 \Longrightarrow \bar{Y}_{. .}=\hat{\alpha}+\hat{\beta} \bar{x}_{.}^{*} \\
\frac{\partial \hat{l}}{\partial \beta} & =0 \Longrightarrow \sum_{t=1}^{n} \sum_{l=1}^{s_{t}}\left(Y_{t l}-\hat{\alpha}-\hat{\beta} x_{t}\right) x_{t}=0 \Longrightarrow \sum_{t=1}^{n} \sum_{l=1}^{s_{t}}\left(Y_{t l}-\bar{Y}_{. .}\right) x_{t}-\hat{\beta} \sum_{t=1}^{n} s_{t}\left(x_{t}-\bar{x}_{.}^{*}\right)^{2} \\
& =0 .
\end{aligned}
$$

De $(A .5)$ e $(A .6)$, obtemos

$$
\hat{\beta}=\frac{\sum_{t=1}^{n} \sum_{l=1}^{s_{t}}\left(Y_{t l}-\bar{Y} . .\right)\left(x_{t}-\bar{x}_{.}^{*}\right)}{\sum_{t=1}^{n} s_{t}\left(x_{t}-\bar{x}_{.}^{*}\right)^{2}}=\frac{\sum_{t=1}^{n} s_{t}\left(Y_{t .}-\bar{Y} . .\right)\left(x_{t}-\bar{x}_{.}^{*}\right)}{\sum_{t=1}^{n} s_{t}\left(x_{t}-\bar{x}_{.}^{*}\right)^{2}} .
$$

Derivando em relação a $\sigma_{u u}$ e $\sigma_{e e}$, obtemos

$$
\begin{gathered}
\frac{\partial \hat{l}}{\partial \sigma_{u u}}=-\frac{1}{2} n \bar{r} \sigma_{u u}^{-1}-\frac{1}{2}\left(-\sigma_{u u}^{-2} \sum_{t=1}^{n} \sum_{j=1}^{r_{t}}\left(X_{t j}-\hat{x}_{t}\right)^{2}\right)=0 \Longrightarrow \\
\hat{\sigma}_{u u}=\frac{\sum_{t=1}^{n} \sum_{j=1}^{r_{t}}\left(X_{t l}-\hat{x}_{t}\right)^{2}}{\sum_{t=1}^{n} r_{t}}
\end{gathered}
$$


e

$$
\begin{gathered}
\frac{\partial \hat{l}}{\partial \sigma_{e e}}=-\frac{1}{2} n \bar{s} \sigma_{e e}^{-1}-\frac{1}{2}\left(-\sigma_{e e}^{-2} \sum_{t=1}^{n} \sum_{l=1}^{s_{t}}\left(Y_{t l}-\hat{\alpha}-\hat{\beta} \hat{x}_{t}\right)^{2}\right)=0 \Longrightarrow \\
\hat{\sigma}_{e e}=\frac{\sum_{t=1}^{n} \sum_{l=1}^{s_{t}}\left(Y_{t l}-\hat{\alpha}-\hat{\beta} \hat{x}_{t}\right)^{2}}{\sum_{t=1}^{n} s_{t}} .
\end{gathered}
$$




\section{Apêndice B}

\section{Teoremas, Lemas e Outros Resultados Básicos}

Colocamos, aqui, os teoremas, lemas e alguns resultados básicos, que, freqüentemente, utilizamos no decorrer da tese.

Apresentamos a seguir as fórmulas, os teoremas e resultados básicos utilizados no cálculo da função de verossimilhança (veja Seção 2.1) e estudos assintóticos dos EMVs; estes resultados aparecem comumente em livros textos, entre os quais citamos apenas os consultados.

\section{B.1 Alguns Teoremas Sobre Matrizes}

Para algumas operações com matrizes utilizamos as seguintes propriedades.

Teorema B.1 (Teorema A3.5 em Muirhead, pg. 578)

Se A é $p \times q$ e $\mathbf{B} q \times p$, então,

$$
\operatorname{det}\left(\mathbf{I}_{p}+\mathbf{A B}\right)=\operatorname{det}\left(\mathbf{I}_{q}+\mathbf{B A}\right)
$$

Teorema B.2 (Teorema A 5.1 em Muirhead, pg. 580) Sejam A e B matrizes não singulares (de dimensões) $p \times p$ e $q \times q$, respectivamente, e sejam $\mathbf{C}, p \times q, e, \mathbf{D}, q \times p$. 
Faça $\mathbf{P}=\mathbf{A}+\mathbf{C B D}$. Então,

$$
\mathrm{P}^{-1}=\mathrm{A}^{-1}-\mathrm{A}^{-1} \mathrm{CB}\left(\mathrm{B}+\mathrm{BDA}^{-1} \mathrm{CB}\right)^{-1} \mathrm{BDA}^{-1}
$$

Teorema B.3 (Teorema A 5.3 em Muirhead, pg. 580/581)

Sejam A uma matriz $p \times p$ não singular e $\mathbf{B}=\mathbf{A}^{-1}$. Particione $\mathbf{A}$ e $\mathbf{B}$ como

$$
\mathbf{A}=\left[\begin{array}{ll}
\mathbf{A}_{11} & \mathbf{A}_{12} \\
\mathbf{A}_{21} & \mathbf{A}_{22}
\end{array}\right], \quad \mathbf{B}=\left[\begin{array}{ll}
\mathbf{B}_{11} & \mathbf{B}_{12} \\
\mathbf{B}_{21} & \mathbf{B}_{22}
\end{array}\right],
$$

onde $\quad \mathbf{A}_{11}$ e $\mathbf{B}_{11}$ são $k \times k ; \mathbf{A}_{12}$ e $\mathbf{B}_{12}$ são $k \times(p-k) ; \quad \mathbf{A}_{21}$ e $\mathbf{B}_{21}$ são $(p-k) \times k$ e $\mathbf{A}_{22}$ é $(p-k) \times(p-k) ;$ supomos que $\mathbf{A}_{11}$ e $\mathbf{A}_{22}$ são não-singulares. Faça

$$
\mathbf{A}_{11.2}=\mathbf{A}_{11}-\mathbf{A}_{12} \mathbf{A}_{22}^{-1} \mathbf{A}_{21}, \quad \mathbf{A}_{22.1}=\mathbf{A}_{22}-\mathbf{A}_{21} \mathbf{A}_{11}^{-1} \mathbf{A}_{12} .
$$

Então,

$$
\begin{aligned}
& \text { a) } \operatorname{det} \mathbf{A}=\operatorname{det} \mathbf{A}_{22} \operatorname{det} \mathbf{A}_{11.2} \\
& \text { b) } \operatorname{det} \mathbf{A}=\operatorname{det} \mathbf{A}_{11} \operatorname{det} \mathbf{A}_{22.1}
\end{aligned}
$$

\section{B.2 Teoremas e Resultados Assintóticos Utilizados no Capítulo 3}

\section{B.2.1 Referências de teoremas}

$\mathrm{Na}$ obtenção dos resultados assintóticos, sob o modelo estrutural normal, dos estimadores alternativos de $\beta$, usamos os seguintes teoremas extraídos, por exemplo, de Sen e Singer (1993) e James (1996):

(1-i) Teorema Central de Limite Clássico: (Teorema 3.3.1 em Sen e Singer (1993, pg. 101))

(1-ii) Método Delta: (Teorema 3.4.4 em Sen e Singer (1993, pg. 131)),

(1-iii) Teorema Central de Limite de Liapounov: (Corolário 2 em James (1996, pg.Central 270) 


\section{B.2.2 Lema de Gleser-Ruppert}

Lema (Gleser, 1981)

Seja $y_{1}, y_{2}, \cdots$ uma seqüência de vetores aleatórios $s$-dimensional, mutuamente independentes, onde $y_{i}$ tem vetor de média zero e matriz de covariância finita $V_{i}$. Se $\lim _{n \rightarrow \infty} n^{-1} \sum_{i=1}^{n} \mathrm{~V}_{i}=\mathrm{V}^{*}$ existe e é finita, então $n^{-\frac{1}{2}} \sum_{i=1}^{n} y_{i} \rightarrow N_{s}\left(0, \mathrm{~V}^{*}\right)$.

Este resultado não é correto da forma em que foi enunciado. Ver o contra-exemplo devido a Ruppert apresentado em Gallo (1982, Capítulo III, Seção 3.1). A conclusão do lema é verdadeira se acrescentarmos algumas condições que envolvem momentos finitos de ordem maior que dois.

\section{B.3 Condições de Regularidade de Mak (1982)}

Reproduzimos, a seguir, as condições de regularidade suficientes para a validade dos resultados assintóticos, estabelecidas por Mak (1982), que serão utilizadas posteriormente na análise das propriedades assintóticas dos estimadores de máxima verossimilhança, no modelo funcional. Cabe lembrar que estas condições se referem, em grande parte, ao comportamento assintótico assumido para a seqüência de parâmetros incidentais, portanto, bastante distintas das condições usualmente adotadas para o caso iid (modelo estrutural); mas são suposições mais fracas que as encontradas, comumente, na literatura.

Mak (1982) estuda as condições sob as quais as equações de verossimilhança têm um máximo num ponto $\widehat{\boldsymbol{\theta}_{n}}$, que converge, em probabilidade, para algum $\theta^{(1)}$ no interior $\Omega^{o}$ de $\Omega$; estabelece a normalidade assintótica de $\widehat{\boldsymbol{\theta}_{n}}$, e baseado neste, constroi um estimador consistente de $\theta^{\circ}$.

(a) Suposições adotadas

As seguintes derivadas existem quase certamente (q.c.) para todo $t, t=1, \ldots, n$

$$
\frac{\partial h_{t}}{\partial \theta_{k}}\left(\mathrm{Z}_{t}, \theta\right)=q_{t k}\left(\mathrm{Z}_{t}, \theta\right), \quad k=1, \ldots, p,
$$




$$
\left.\frac{\partial^{2} h_{t}}{\partial \theta_{l} \partial \theta_{k}}\left(\mathbf{Z}_{t}, \theta\right)\right)=q_{t k l}\left(\mathbf{Z}_{t}, \theta\right), \quad l, k=1, \ldots, p
$$

sendo

$$
h_{t}\left(\mathrm{Z}_{t}, \theta\right)=\ln \left(f_{t}\left[\left(\mathrm{Z}_{t}, \theta\right), g_{t}\left(\mathrm{Z}_{t} ; \theta\right)\right]\right) .
$$

( b) Sejam

$$
g_{t}\left(\mathrm{Z}_{t}, \theta\right)=\left(q_{t 1}, \ldots, q_{t p}\right)^{\prime}
$$

$\mathrm{e}$

$$
\mathrm{A}_{n}(\theta)=\left(\frac{\sum_{t=1}^{n} q_{t k l}\left(\mathbf{Z}_{t}, \theta\right)}{n}\right)_{k l}
$$

uma matriz simétrica aleatória.

Defina

$$
\Psi(\theta)=n^{-1} \sum_{t}^{n} E^{0}\left(h_{t}\left(Z_{t}, \theta\right)\right),
$$

em que $E^{0}()=.E\left(. \mid \theta^{(0)}, x_{t}^{(0)}\right)$, e notação similar para $V^{0}$.

Transcrevemos a seguir as condições de regularidade que garantam a normalidade assintótica e consistência do estimador baseado em $\widehat{\boldsymbol{\theta}_{n}}$. Veja, também, Vilca-Labra (1996) e Gimenez (1997).

Seja $\theta^{(1)} \in \Omega^{(0)}$ e considere as seguintes condições:

(C.1) A função $\psi(\theta)$ converge uniformemente (em $\theta$ ) para uma função $\bar{h}(\theta)$ numa vizinhança de $\theta^{(1)}$, e $\theta^{(1)}$ é um máximo local de $\bar{h}(\theta)$.

(C.2) A matriz $\left.\left(\frac{\partial \bar{h}^{2}}{\partial \theta_{j} \partial \theta_{i}}\right)\right|_{\theta^{(1)}}, \mathrm{p} \times \mathrm{p}$, é não singular.

(C.3) Para cada $\theta \in \Omega^{0}$, existem $\delta>0$ e as funções $d_{t}\left(\mathbf{Z}_{t}\right)$ e $d_{t k l}\left(\mathrm{Z}_{t}\right)$ tal que:

$$
\left|h_{t}\left(X_{t}, \theta^{\prime}\right)\right|<d_{t}\left(\mathbf{Z}_{t}\right), \quad\left|q_{t k l}\left(\mathbf{Z}_{t}, \theta^{\prime}\right)\right|<d_{t k l}\left(\mathbf{Z}_{t}\right) \quad \text { q.c. }
$$

para todo $\theta^{\prime} \in S_{\theta, \delta^{\prime}}=\left\{\theta^{\prime}:\left\|\theta^{\prime}-\theta\right\|<\delta\right\} \subseteq \Omega^{0}$

e $\limsup \left[\frac{\sum_{t=1}^{n} E^{0}\left[d_{t}\left(\mathbf{Z}_{t}\right)\right]^{2}}{n}\right]<\infty, \limsup \left[\frac{\sum_{t=1}^{n} E^{0}\left[d_{t k l}\left(\mathbf{Z}_{t}\right)\right]^{2}}{n}\right]<\infty$, 
(C.4) $\limsup \left[\frac{\sum_{t=1}^{n} E^{0}\left|q_{t k}\left(\mathbf{Z}_{t}\right)\right|}{n}\right]<\infty$, para cada $k=1, \ldots, p$.

(C.5) $0<\liminf \| E^{0}\left(A_{n}\left(\theta^{(1)}\right)\left\|\leq \limsup E^{0}\right\| A_{n}\left(\theta^{(1)} \|<\infty, \liminf \left[\operatorname{det} E^{0}\left(A_{n}\left(\theta^{(1)}\right)\right)\right]>\right.\right.$ 0 , onde $\|\cdot\|$ de uma matriz $\mathrm{A}=\left[a_{i j}\right]_{n \times m}$ é definido como $\left(\sum_{n}^{i=1} \sum_{m}^{j=1} a_{i j}^{2}\right)^{\frac{1}{2}}$.

(C.6) Dado $\varepsilon>0, \exists \delta>0$ tal que $\limsup \left|\frac{\sum_{t=1}^{n} E^{0}\left(\sup \left[q_{i k l}\left(\mathrm{Z}_{t}, \theta\right):\left\|\theta-\theta^{(1)}\right\|<\delta\right]-q_{i k l}\left(\mathrm{Z}_{t}, \theta^{(1)}\right)\right)}{n}\right|<' \varepsilon$; e o mesmo é verdade quando sup é substituído por inf.

(C.7) $\exists$ um $\gamma>0$ tal que:

$$
\frac{1}{n^{1+\frac{\gamma}{2}}} \sum_{t=1}^{n} E^{0}\left|q_{i k l}\left(\mathbf{Z}_{t}, \theta^{(1)}\right)\right|^{2+\gamma} \longrightarrow 0, \text { quando } n \longrightarrow \infty, \text { para } \quad k=1, \ldots, p .
$$

$$
0<\liminf \frac{1}{n} \sum_{t=1}^{n} \operatorname{Var}^{0}\left[q_{i k l}\left(\mathbf{Z}_{t}, \boldsymbol{\theta}^{(1)}\right)\right] \leq \limsup \frac{1}{n} \sum_{t=1}^{n} \operatorname{Var}^{0}\left[q_{i k l}\left(\mathbf{Z}_{t}, \boldsymbol{\theta}^{(1)}\right)\right]<\infty .
$$

Sob as condições acima, Mak (1982) estabelece e demontra os seguintes teoremas (Teorema 3.1A em Mak (1982)):

Teorema B.4 Se $\theta^{(1)} \in \Omega^{0}$ e as condições (C.1) a (C.4) forem satisfeitas, então:

(R1) Com probabilidade tendendo a um $\Psi(\theta)$ em (B.4) tem um máximo $\widehat{\theta}_{n}$, tal que $\widehat{\theta}_{n}$ tende em probabilidade para $\theta^{(1)}$ quando $n \rightarrow \infty$.

(R2) $n^{-1} \sum_{t=1}^{n} E^{0}\left[q_{t}\left(\mathrm{Z}_{t}, \theta^{(1)}\right)\right]=0$.

Teorema B.5 Se $\theta^{(1)} \in \Omega^{0}$ satisfaz (B.5) e as condições (C.3) a (C.6) forem satisfeitas, então:

(R3) com probabilidade um a equação:

$$
\sum_{t=1}^{n} q_{t}\left(\mathbf{Z}_{t}, \theta\right)=0
$$


tem uma raiz $\bar{\theta}_{n}$ que converge em probabilidade para $\theta^{(1)}$. Além disso, $\bar{\theta}_{n}$ é único, no sentido de, se $\bar{\theta}_{n}^{\prime}$ é também uma raíz de (B.5) e $\bar{\theta}_{n}^{\prime}$ tende em probabilidade para $\theta^{(1)}$, então com probabilidade tendendo a um, $\bar{\theta}_{n}{ }^{\prime}=\bar{\theta}_{n}$.

Teorema B.6 Supomos que $\theta^{(1)} \in \Omega^{0}$ satisfaz (R1) dado em (B.5). Sob as suposições (C.3) - (C.8), com probabilidade tendendo a um, existe uma raíz $\hat{\theta}_{n}$ de (R3) dado em (B.6) que é assintoticamente normal com média $\theta^{(1)}$ e variância (veja as notações desta variância na Seção 4.2) $n^{-1}\left\{E\left[A_{n}\left(\theta^{1}\right)\right]\right\}^{-1} V_{n}\left\{E\left[\left(A_{n}\left(\theta^{1}\right)\right]\right\}^{-1}\right.$.

As provas detalhadas dos resultados acima podem ser encontradas em Gimenez (1997). Observações sobre as condições dadas e das utilidades dos teoremas.

1. As condições (C.1) e (C.2) podem ser substituídas pela suposição $\Psi\left(\boldsymbol{\theta}^{(1)}\right)>\Psi(\boldsymbol{\theta})$ para todo $\theta \in S_{\theta^{\prime}, \delta} \theta^{(1)}$, para algum $\delta>0$ e todo n suficientemente grande.

2. Se as terceiras derivadas de $h_{t}\left(\mathbf{Z}_{t}, \boldsymbol{\theta}\right)$ existirem q.c., então a condição (C.6) pode ser substituída pela $\left(C^{\prime} .6\right)$, dada abaixo, que é mais fácil de se verificar em algumas situações.

$\left(C^{\prime} .6\right)$ Existe uma função $d_{t k l m}\left(\mathbf{Z}_{t}\right)$, tal que: $\left|\frac{\partial}{\partial \theta_{m}} q_{t k l}\left(\mathbf{Z}_{t}, \theta\right)\right|<\left|d_{t k l m}\left(\mathbf{Z}_{t}\right)\right|$ numa vizinhança de $\theta$, e limsup $\frac{E^{0} \mid d_{t k l m}\left(\mathbf{Z}_{t} \mid\right.}{n}<\infty$, para $k, l, m=1, \ldots, p$.

3. As propriedades assintóticas de qualquer estimador dependem claramente do comportamento assintótico da sequência $\left\{x_{t}^{(0)}\right\}_{t \geq 1}$. As expectâncias nas condições (C.3) - (C.8) são tomadas quando $x_{t}^{(0)}$ é observado e, portanto, são funções de $x_{t}^{(0)}$. As condições (C.1) - (C.8) são, consequentemente, impostas sobre o comportamento assintótico da seqüência $x_{t}^{(0)}$.

O $\theta^{(1)}$ no Teorema B.4 maximiza $\Psi(\theta)$ para todo n grande. Seu valor, em geral, depende do verdadeiro vetor de parâmetros estruturais $\theta^{(0)}$ e dos verdadeiros parâmetros incidentais $x_{t}^{0}$, pois as expectâncias são tomadas sob $\theta^{(0)}$ e $x_{t}^{(0)}$. Porém, em algumas situações, é possível achar estimadores $g_{t}\left(Z_{t}, \theta\right)$ tal que o $\theta^{(1)}$ no Teorema B.4 é determinado apenas por $\boldsymbol{\theta}^{(0)}$ e é independente dos valores de $x_{t}^{0}$. 


\section{Apêndice C}

\section{Condições do TCL de Liapounov e do Gleser- Ruppert}

\section{C.1 Cálculo de $E\left(R_{3 t}\right)^{3}$ e $E\left(R_{3 t}\right)^{4}$}

Os cálculos apresentados neste Apêndice foram utilizados no Capítulo 3, ou, mais especificamente, na demonstração de que os quartos momentos das variáveis $U_{3 t}, U_{2 t}$ e $U_{1 t} ; t=$ $1, \ldots, n$ são finitos - condição imposta por Ruppert (Gallo 1982) para validar o resultado do Lema de Gleser (1981). Esses cálculos também foram aproveitados para mostrar que essas seqüências $\left(\left\{U_{i t}\right\}_{(t \geq 1)}, t=1,2 e 3\right)$ satisfazem as condições do teorema central do limite de Liapounov e para calcular as suas variâncias.

Reescrevendo o modelo considerado, temos

$$
\begin{array}{rlrl}
\left(\begin{array}{lll}
x_{t} & e_{t} & u_{t j}
\end{array}\right)^{\prime} \sim N\left[\left(\begin{array}{lll}
\mu_{x} & 0 & 0
\end{array}\right)^{\prime}, \operatorname{diag}\left\{\sigma_{x x}, \sigma_{e e}, \sigma_{u u}\right\}\right], & \\
X_{t_{j}} & =x_{t}+u_{t_{j}}, & Y_{t} & =\alpha+\beta x_{t} \\
Y_{t} & =y_{t}+e_{t}, & & \bar{X}_{t .}=\sum_{j=1}^{r_{t}} \frac{X_{t_{j}}}{r_{t}}
\end{array}
$$

onde

$$
\left(\begin{array}{lll}
x_{t} & e_{t} & \bar{u}_{t .}
\end{array}\right)^{\prime} \sim N\left[\left(\begin{array}{ccc}
\mu_{x} & 0 & 0
\end{array}\right)^{\prime}, \operatorname{diag}\left\{\sigma_{x x}, \sigma_{e e}, \frac{\sigma_{u u}}{r_{t}}\right\}\right]
$$


Sendo

$$
\begin{aligned}
R_{3 t} & =\sum_{j=1}^{r_{t}}\left(X_{t_{j}}-\bar{X}_{t .}\right)^{2}=\sum_{j=1}^{r_{t}}\left(u_{t_{j}}-\bar{u}_{t .}\right)^{2} \\
& =\sum_{j=1}^{r_{t}} u_{t_{j}}^{2}-r_{t} \bar{u}_{t .}^{2}=\sum_{j=1}^{r_{t}} u_{t_{j}}^{2}-\frac{1}{r_{t}}\left(\sum_{j=1}^{r_{t}} u_{t_{j}}\right)^{2},
\end{aligned}
$$

temos

$$
R_{3 t}=0 \quad \text { para } r_{t}=1 \quad \text { e } \quad R_{3 t} \sim\left(\sigma_{u u}\right) \chi_{r_{t}-1}^{2} \text { para } r_{t}>1 .
$$

Se $Z \sim N(0,1)$, temos

$$
E(Z)=0, \quad E\left(Z^{2}\right)=1, \quad E\left(Z^{4}\right)=3, \quad E\left(Z^{6}\right)=15, \quad E\left(Z^{8}\right)=105 .
$$

De (C.1), (C.2) e (C.4), temos que:

$$
\begin{aligned}
& E\left(R_{3 t}\right)=\left(r_{t}-1\right) \sigma_{u u}, \quad \operatorname{Var}\left(R_{3 t}\right)=2\left(r_{t}-1\right) \sigma_{u u}^{2} \quad e \\
& E\left(R_{3 t}^{2}\right)=\left(r_{t}^{2}-1\right) \sigma_{u u}^{2} .
\end{aligned}
$$

A seguinte fórmula (Polinômio de Leibniz) será usada para desenvolver potências envolvendo somatórios. O polinômio de Leibniz é uma genaralização do binômio de Newton e pode ser enunciado como segue:

$$
\left(x_{1}+x_{2}+\cdots+x_{p}\right)^{n}=\sum \frac{n !}{\alpha_{1} ! \alpha_{2} ! \ldots \alpha_{p} !} x_{1}^{\alpha_{1}} x_{2}^{\alpha_{2}} \ldots x_{p}^{\alpha_{n}},
$$

estendendo-se o somatório a todos os valores inteiros não-negativos de $\alpha_{1}, \alpha_{2}, \ldots \alpha_{p}$ tais que $\alpha_{1}+\alpha_{2}+\cdots+\alpha_{p}=n$ (para mais detalhes ver Morgado et alli, 1991).

\section{C.1.1 Cálculos preliminares}

(1) Cálculo de $E\left(\sum_{j=1}^{r_{t}} u_{t_{j}}^{2}\right)^{4}$.

Usando a fórmula de Leibniz (C.6), e excluindo os termos que zeram $\left(E\left(u_{T_{j}}^{k}\right)=0\right.$, para $k$ impar) - e somando as igualdades $\left(E\left(u_{T_{j}}^{p} u_{T_{i}}^{q}\right)=E\left(u_{T_{j}}^{q} u_{T_{i}}^{p}\right)\right)$, temos 


$$
\begin{aligned}
E\left(\sum_{j=1}^{r_{t}} u_{t_{j}}^{2}\right)^{4}= & E\left\{\sum_{j=1}^{r_{t}} u_{t_{j}}^{8}\right\}+8 I_{r_{t}}(2) \sum_{j=1}^{r_{t}-1} \sum_{i>j}^{r_{t}} E\left(u_{t_{j}}^{6} \cdot u_{t_{i}}^{2}\right) \\
& +6 I_{r_{t}}(2) \sum_{j=1}^{r_{t}} \sum_{i>j}^{r_{t}} E\left(u_{t_{j}}^{4} \cdot u_{t_{i}}^{4}\right)+36 I_{r_{t}}(3) \sum_{k=1}^{r_{t}-2} \sum_{j>k}^{r_{t}-1} \sum_{i>j}^{r_{t}} E\left(u_{t_{k}}^{4} \cdot u_{t_{j}}^{2} \cdot u_{t_{i}}^{2}\right) \\
& +24 I_{r_{t}}(4) \sum_{l=1}^{r_{t}-3} \sum_{k>l}^{r_{t}-2} \sum_{j>k}^{r_{t}-1} \sum_{i>j}^{r_{t}} E\left(u_{t_{l}}^{2} \cdot u_{t_{k}}^{2} \cdot u_{t_{j}}^{2} \cdot u_{t_{i}}^{2}\right)
\end{aligned}
$$

onde

$$
I_{r_{i}}(r)= \begin{cases}1, & \text { se } r_{t} \geq r \\ 0, & \text { se } r_{t}<r .\end{cases}
$$

De (C.4), e para $1 \leq l<k<j<i \leq r_{t}$, temos

$$
\begin{aligned}
& E\left(u_{t_{j}}^{8}\right)=105 \sigma_{u u}^{4} ; \quad E\left(u_{t_{j}}^{6} \cdot u_{t_{i}}^{2}\right)=5 \sigma_{u u}^{4} ; \\
& E\left(u_{t_{j}}^{4} \cdot u_{t_{i}}^{4}\right)=9 \sigma_{u u}^{4} ; \quad E\left(u_{t_{k}}^{4} \cdot u_{t_{j}}^{2} \cdot u_{t_{i}}^{2}\right)=3 \sigma_{u u}^{4} ; \\
& E\left(u_{t_{l}}^{2} \cdot u_{t_{k}}^{2} \cdot u_{t_{j}}^{2} \cdot u_{t_{i}}^{2}\right)=\sigma_{u u}^{4} .
\end{aligned}
$$

Substituindo as igualdades (C.8) em (C.7), temos

$$
\begin{aligned}
E\left(\sum_{j=1}^{r_{t}} u_{t_{j}}^{2}\right)^{4}= & {\left[105 r_{t}+87 r_{t}\left(r_{t}-1\right) I_{r_{t}}(2)+18 r_{t}\left(r_{t}-1\right)\left(r_{t}-2\right) I_{r_{t}}(3)\right.} \\
& \left.+r_{t}\left(r_{t}-1\right)\left(r_{t}-2\right)\left(r_{t}-3\right) I_{r_{t}}(4)\right] \sigma_{u u}^{4}
\end{aligned}
$$

Analogamente, temos

$$
\begin{aligned}
&(2) E\left(\sum_{j=1}^{r_{t}} u_{t_{j}}^{2}\right)^{3}=\left[15 r_{t}+9 r_{t}\left(r_{t}-1\right) I_{r_{t}}(2)\right. \\
&\left.+r_{t}\left(r_{t}-1\right)\left(r_{t}-2\right) I_{r_{t}}(3)\right] \sigma_{u u}^{3}, \\
&(3) E\left(\sum_{j=1}^{r_{t}} u_{t_{j}}^{4}\right)^{2}=\left[105 r_{t}+9 r_{t}\left(r_{t}-1\right) I_{r_{t}}(2)\right] \sigma_{u u}^{3} .
\end{aligned}
$$


(4) Cálculo de $E\left[\left(\sum_{k=1}^{r_{t}} u_{t_{k}}^{4}\right)\left(\sum_{j=1}^{r_{t}-1} \sum_{i>j}^{r_{t}} u_{t_{j}}^{2} \cdot u_{t_{i}}^{2}\right)\right]=S 3$

Temos que

$$
\begin{aligned}
S 3= & \sum_{k=1}^{r_{t}}\left\{\sum _ { j = 1 } ^ { r _ { t } - 1 } \left[\left(E\left(u_{t_{k}}^{6}\right) E\left(\sum_{i>j}^{r_{t}-1} u_{t_{i}}^{2}\right)\right) I_{(j=k)}+\left(E\left(u_{t_{k}}^{4}\right) E\left(u_{t_{j}}^{2}\right) E \sum_{i>j}^{r_{t}} u_{t_{i}}^{2}\right) I_{(k<j)}\right.\right. \\
& \left.\left.+\left(E\left(u_{t_{k}}^{6} \cdot u_{t_{j}}^{2}\right)+E\left(u_{t_{j}}^{2}\right) E\left(u_{t_{k}}^{4}\right) \sum_{i>j}^{r_{t}} E\left(u_{t_{i}}^{2}\right)\right) I_{(k>j)}\right]\right\} \\
= & \sum_{k=1}^{r_{t}}\left\{\sum _ { j = 1 } ^ { r _ { t } - 1 } \left[15 \sigma_{u u}^{4}\left(r_{t}-j\right) I_{(k=j)}+3 \sigma_{u u}^{4}\left(r_{t}-j\right) 1_{(k<j)}\right.\right. \\
& \left.\left.+\left(15+3\left(r_{t}-j-1\right)\right) \cdot \sigma_{u u}^{4} I_{(k>j)}\right]\right\} \\
= & \left.\sigma_{u u}^{4} \sum_{k=1}^{r_{t}}\left\{\sum_{j=1}^{r_{t}-1}\left[12\left(r_{t}-j\right) I_{(j=k)}+3\left(r_{t}-j\right) I_{(k=j)}+I_{(k<j)}+1_{(k>j)}\right)+12 I_{(k>j)}\right]\right\} \\
= & \frac{3 r_{t}\left(r_{t}-1\right)\left(r_{t}+8\right)}{2} \sigma_{u u}^{4} I_{r_{t}}(2),
\end{aligned}
$$

onde

$$
I_{(k \geq j)}=\left\{\begin{array}{ll}
1, & \text { se } k \geq j \\
0, & \text { se } k<j
\end{array},\right.
$$

e de modo similar definimos $\quad I_{(k \leq j)}$ e $I_{(k=j)}$.

Analogamente, temos

$$
\begin{aligned}
& E\left[\left(\sum_{k=1}^{r_{t}} u_{t_{k}}^{2}\right)\left(\sum_{j=1}^{r_{t}-1} \sum_{i>j}^{r_{t}} u_{t_{j}}^{2} \cdot u_{t_{i}}^{2}\right)\right]=\frac{1}{2} r_{t}\left(r_{t}-1\right)\left(r_{t}+4\right) \sigma_{u u}^{3} I_{r_{t}}(2), \\
& E\left[\left(\sum_{k=1}^{r_{t}} u_{t_{k}}^{2}\right)\left(\sum_{j=1}^{r_{t-1}} \sum_{i>j}^{r_{t}} u_{t_{j}}^{4} \cdot u_{t_{i}}^{2}\right)\right]=\frac{3}{2} r_{t}\left(r_{t-1}\right)\left(r_{t}+6\right) \sigma_{u u}^{4}
\end{aligned}
$$

(7) Cálculo de $E\left[\left(\sum_{j=1}^{r_{t}} u_{t_{j}}^{2}\right)\left(\sum_{j=1}^{r_{t}} u_{t_{j}}^{6}\right)\right]=\mathrm{S} 4$

$$
\begin{aligned}
S 4 & =E\left[\sum_{j=1}^{r_{t}} u_{t_{j}}^{8}+I_{r_{t}}(2) \sum_{j=1}^{r_{t}-1} \sum_{i>j}^{r_{t}}\left(u_{t_{j}}^{2} \cdot u_{t_{i}}^{6}+u_{t_{j}}^{6} \cdot u_{t_{i}}^{2}\right)\right] \\
& =\left[105 r_{t}+15 r_{t}\left(r_{t}-1\right) I_{r_{t}}(2)\right] \sigma_{u u}^{4} .
\end{aligned}
$$


Analogamente, temos

(8) $E\left[\left(\sum_{j=1}^{r_{t}} u_{t_{j}}^{2}\right)\left(\sum_{j=1}^{r_{t}} u_{t_{j}}^{4}\right)\right]=\left[15 r_{t}+3 r_{t}\left(r_{t}-1\right) I_{r_{t}}(2)\right] \sigma_{u u}^{3}$

(9) Cálculo de $E\left\{\left(\sum_{j=1}^{r_{t}} u_{t_{j}}^{2}\right)^{2}\left(\sum_{j=1}^{r_{t}} u_{t_{j}}\right)^{4}\right\}=S 5$.

Usando a fórmula de Leibniz, temos que

$$
\begin{aligned}
\left(\sum_{j=1}^{r_{t}} u_{t_{j}}\right)^{4}= & \sum_{j=1}^{r_{t}} u_{t_{j}}^{4}+4 \sum_{j=1}^{r_{t}-1} \sum_{i>j}^{r_{t}}\left(u_{t_{j}}^{3} \cdot u_{t_{i}}+u_{t_{j}} \cdot u_{t_{i}}^{3}\right) I_{r_{t}}(2)+6 \sum_{j=1}^{r_{t}-1} \sum_{i>j}^{r_{t}} u_{t_{j}}^{2} \cdot u_{t_{i}}^{2} I_{r_{t}}(2) \\
& +12 \sum_{k=1}^{r_{t}-2} \sum_{j>k}^{r_{t}-1} \sum_{i>j}^{r_{t}}\left(u_{t_{k}}^{2} \cdot u_{t_{j}} \cdot u_{t_{i}}+u_{t_{k}} \cdot u_{t_{j}}^{2} \cdot u_{t_{i}}+u_{t_{k}} \cdot u_{t_{j}} \cdot u_{t_{i}}^{2}\right) I_{r_{t}}(3) \\
& +24 \sum_{l=1}^{r_{t}-3} \sum_{k>l}^{r_{t}-2} \sum_{j>k}^{r_{t}-1} \sum_{i>j}^{r_{t}}\left(u_{t_{l}} \cdot u_{t_{k}} \cdot u_{t_{j}} \cdot u_{t_{i}}\right) I_{r_{t}}(4) .
\end{aligned}
$$

De (C.17), e excluindo os termos, na expressão anterior, que resultam em valores esperados iguais a zero, quando multiplicados por $\left(\sum_{j=1}^{r_{t}} u_{t_{j}}^{2}\right)^{2}$, temos

$$
S 5=E\left\{\left(\sum_{j=1}^{r_{t}} u_{t_{j}}^{2}\right)^{2}\left[\sum_{j=1}^{r_{t}} u_{t_{j}}^{4}+6 \sum_{j=1}^{r_{t}-1} \sum_{i>j}^{r_{t}}\left(u_{t_{j}}^{2} \cdot u_{t_{i}}^{2}\right) 1_{r_{t}}(2)\right]\right\} .
$$

Mas,

$$
\begin{aligned}
\sum_{j=1}^{r_{t}} u_{t_{j}}^{4}+6 \sum_{j=1}^{r_{t}-1} \sum_{i>j}^{r_{t}}\left(u_{t_{j}}^{2} \cdot u_{t_{i}}^{2}\right) I_{r_{t}}(2)= & 3\left(\sum_{j=1}^{r_{t}} u_{t_{j}}^{4}+2 \sum_{j=1}^{r_{t}-1} \sum_{i>j}^{r_{t}}\left(u_{t_{j}}^{2} \cdot u_{t_{i}}^{2}\right) I_{r_{t}}(2)\right) \\
& -2 \sum_{j=1}^{r_{t}} u_{t_{j}}^{4} \\
= & 3\left(\sum_{j=1}^{r_{t}} u_{t_{j}}^{2}\right)^{2}-2 \sum_{j=1}^{r_{t}} u_{t_{j}}^{4} .
\end{aligned}
$$

Substituindo (C.19) em (C.18), temos

$$
S 5=3 E\left[\left(\sum_{j=1}^{r_{t}} u_{t_{j}}^{2}\right)^{4}\right]-2 E\left\{\left(\sum_{j=1}^{r_{t}} u_{t_{j}}^{2}\right)^{2}\left(\sum_{j=1}^{r_{t}} u_{t_{j}}^{4}\right)\right\}
$$


Mas, de (C.11) e (C.12), temos:

$$
\begin{aligned}
E\left[\left(\sum_{j=1}^{r_{t}} u_{t_{j}}^{2}\right)\right]^{2}\left[\sum_{j=1}^{r_{t}} u_{t_{j}}^{4}\right] & =\left\{E\left[\left(\sum_{j=1}^{r_{t}} u_{t_{j}}^{4}\right)+2 \sum_{j=1}^{r_{t}-1} \sum_{i>j}^{r_{t}} u_{t_{j}}^{2} \cdot u_{t_{i}}^{2} I_{r_{t}}(2)\right]\left[\sum_{j=1}^{r_{t}} u_{t_{j}}^{4}\right]\right\} \\
& =\left[105 r_{t}+3 r_{t}\left(r_{t}-1\right)\left(r_{t}+11\right) \cdot I_{r_{t}}(2)\right] \sigma_{u u}^{4} .
\end{aligned}
$$

Substituindo $(C .21)$ e $(C .9)$ em $(C .18)$, obtemos S5.

(10) Cálculo de $E\left\{\left(\sum_{l=1}^{r_{t}}\left(u_{t_{k}}^{2}\right)\left(\sum_{k=1}^{r_{t}-2} \sum_{j>k}^{r_{t}-1} \sum_{i>j}^{r_{t}} u_{t_{k}}^{2} \cdot u_{t_{j}}^{2} \cdot u_{t_{i}}^{2}\right)\right\}=S 6\right.$.

Temos, pela fórmula de Leibniz, que

$$
\begin{aligned}
\left(\sum_{j=1}^{r_{t}} u_{t_{j}}^{2}\right)^{3}= & \sum_{j=1}^{r_{t}} u_{t_{j}}^{6}+3 \sum_{j=1}^{r_{t}} \sum_{i>j}^{r_{t}}\left(u_{t_{j}}^{4} \cdot u_{t_{i}}^{2}+u_{t_{j}}^{2} \cdot u_{t_{i}}^{4}\right) I_{r_{t}}(2) \\
& +6\left(\sum_{k=1}^{r_{t}-2} \sum_{j>k}^{r_{t}-1} \sum_{i>j}^{r_{t}}\left(u_{t_{k}}^{2} \cdot u_{t_{j}}^{2} \cdot u_{t_{i}}^{2}\right) I_{r_{t}}(3)\right) .
\end{aligned}
$$

De (C.22) temos

$$
\begin{aligned}
\sum_{k=1}^{r_{t}-2} \sum_{j>k}^{r_{t}-1} \sum_{i>j}^{r_{t}}\left(u_{t_{k}}^{2} \cdot u_{t_{j}}^{2} \cdot u_{t_{i}}^{2}\right) I_{r_{t}}(3)= & \frac{1}{6}\left\{\left(\sum_{j=1}^{r_{t}} u_{t_{j}}^{2}\right)^{3}-\sum_{j=1}^{r_{t}} u_{t_{j}}^{6}\right. \\
& \left.-3 \sum_{j=1}^{r_{t}-1} \sum_{i>j}^{r_{t}}\left(u_{t_{j}}^{4} \cdot u_{t_{i}}^{2}+u_{t_{i}}^{2} u_{t_{i}}^{4}\right)\right\} I_{r_{t}}(3) .
\end{aligned}
$$

Substituindo (C.23) em S6, temos

$$
\begin{aligned}
S 6 & =\frac{1}{6}\left\{E\left(\sum_{j=1}^{r_{t}} u_{t_{j}}^{2}\right)^{4}-E\left[\left(\sum_{j=1}^{r_{t}} u_{t_{j}}^{2}\right)\left(\sum_{j=1}^{r_{t}} u_{t_{j}}^{6}\right)\right]\right. \\
& \left.-6 I_{r_{t}}(2) E\left[\left(\sum_{k=1}^{r_{t}} u_{t_{k}}^{2}\right)\left(\sum_{j=1}^{r_{t}-1} \sum_{i>j}^{r_{t}} u_{t_{j}}^{4} \cdot u_{t_{i}}^{2}\right)\right]\right\} .
\end{aligned}
$$

Substituindo (C.7), (C.14) e (C.15) em (C.24), temos 


$$
\begin{aligned}
S 6= & E\left\{\left(\sum_{l=1}^{r_{t}} u_{t_{k}}^{2}\right)\left(\sum_{k=1}^{r_{t}-2} \sum_{j>k}^{r_{t}-1} \sum_{i>j}^{r_{t}} u_{t_{k}}^{2} \cdot u_{t_{j}}^{2} \cdot u_{t_{i}}^{2}\right)\right\} \\
= & {\left[\frac{3}{2}\left(2-r_{t}\right) r_{t}\left(r_{t}-1\right) I_{r_{t}}(2)+3 r_{t}\left(r_{t}-1\right)\left(r_{t}-2\right) \cdot I_{r_{t}}(3)\right.} \\
& \left.+\frac{1}{6} r_{t}\left(r_{t}-1\right)\left(r_{t}-2\right)\left(r_{t}-3\right) I_{r_{t}}(4)\right] \sigma_{u u}^{4} .
\end{aligned}
$$

(11) Cálculo de $E\left[\left(\sum_{j=1}^{r_{t}} u_{t_{j}}^{2}\right)\left(\sum_{i=1}^{r_{t}} u_{t_{i}}\right)^{6}\right]=S 7$.

Excluindo de $\left(\sum_{i=1}^{r_{t}} u_{t_{i}}\right)^{6}$ os termos cujos produtos com $\left(\sum_{j=1}^{r_{t}} u_{t_{j}}^{2}\right)$, resultam em valores esperados iguais a zeros, temos

$$
\begin{aligned}
S 7= & E\left[\left(\sum_{j=1}^{r_{t}} u_{t_{j}}^{2}\right)\left(\sum_{i=1}^{r_{t}} u_{t_{i}}^{6}\right)\right]+30 E\left[\left(\sum_{l=1}^{r_{t}} u_{t_{l}}^{2}\right)\left(\sum_{j=1}^{r_{t}-1} \sum_{i>j}^{r_{t}}\left(u_{t_{j}}^{4} \cdot u_{t_{i}}^{2}\right) I_{r_{t}}(2)\right)\right] \\
& +90 E\left[\left(\sum_{l=1}^{r_{t}} u_{t_{l}}^{2}\right)\left(\sum_{k=1}^{r_{t}-2} \sum_{j>k}^{r_{t}-1} \sum_{i>j}^{r_{t}}\left(u_{t_{k}}^{2} \cdot u_{t_{j}}^{2} \cdot u_{t_{i}}^{2}\right) I_{r_{t}}(3)\right)\right] .
\end{aligned}
$$

Substituindo (C.15), (C.14) e (C.25) em (C.26), temos

$$
\begin{aligned}
S 7= & E\left[\left(\sum_{j=1}^{r_{t}} u_{t_{j}}^{2}\right)\left(\sum_{i=1}^{r_{t}} u_{t_{i}}\right)^{6}\right] \\
= & \left\{105 r_{t}+15 r_{t}\left(r_{t}-1\right)\left(3 r_{t}+19\right) 1_{r_{t}}(2)+135 r_{t}\left(r_{t}-1\right)\left(r_{t}-2\right) I_{r_{t}}(3)\right. \\
& \left.+15 r_{t}\left(r_{t}-1\right)\left(r_{t}-2\right)\left(r_{t}-3\right) I_{r_{t}}(4)\right\} \sigma_{u u}^{4}
\end{aligned}
$$

Analogamente, temos

$$
E\left[\left(\sum_{j=1}^{r_{t}} u_{t_{j}}^{2}\right)\left(\sum_{i=1}^{r_{t}} u_{t_{i}}\right)^{4}\right]=E\left[\left(\sum_{j=1}^{r_{t}} u_{t_{j}}^{2}\right)\left(\sum_{j=1}^{r_{t}} u_{t_{j}}^{4}+6 \sum_{j=1}^{r_{t}-1} \sum_{i>j}^{r_{t}}\left(u_{t_{j}}^{2} \cdot u_{t_{i}}^{2}\right) I_{r_{t}}(2)\right)\right] .
$$

Substituindo (C.13) e (C.16) em (C.28) temos:

(12) $E\left[\left(\sum_{j=1}^{r_{t}} u_{t_{j}}^{2}\right)\left(\sum_{i=1}^{r_{t}} u_{t_{i}}\right)^{4}\right]=\left[15 r_{t}+3 r_{t}\left(r_{t}-1\right)\left(r_{t}+5\right) 1_{r_{t}}(2)\right]$. 
(13) Cálculo de $E\left[\left(\sum_{j=1}^{r_{t}} u_{t_{j}}^{2}\right)^{3}\left(\sum_{i=1}^{r_{t}} u_{t_{i}}\right)^{2}\right]=S 08$.

$$
S 08=E\left[\left(\sum_{j=1}^{r_{t}} u_{t_{j}}^{2}\right)^{3}\left(\sum_{j=1}^{r_{t}} u_{t_{j}}^{2}+2 \sum_{j=1}^{r_{t}-1} \sum_{i>j}^{r_{t}} u_{t_{j}} \cdot u_{t_{i}}\right)\right]=E\left(\sum_{j=1}^{r_{t}} u_{t_{j}}^{2}\right)^{4}
$$

Analogamente, temos

(14) $E\left[\left(\sum_{j=1}^{r_{t}} u_{t_{j}}^{2}\right)^{2}\left(\sum_{i=1}^{r_{t}} u_{t_{i}}\right)^{2}\right]=E\left(\sum_{j=1}^{r_{t}} u_{t_{j}}^{2}\right)^{3}$.

(15) Cálculo de $E\left(\bar{u}_{t .}^{2}\right)^{4}$.

$$
\begin{aligned}
E\left(\bar{u}_{t .}^{2}\right)^{4} & =E\left(\bar{u}_{t .}^{8}\right)=E\left\{\left(\frac{\bar{u}_{t .}}{\sqrt{\frac{\sigma_{u u}}{r_{t}}}}\right)^{8}\left(\sqrt{\frac{\sigma_{u u}}{r_{t}}}\right)^{8}\right\}=\left(\frac{\sigma_{u u}^{4}}{r_{t}^{4}}\right) E\left[\left(Z_{\bar{u}_{t .}}\right)^{8}\right] \\
& =105 r_{t}^{-4} \sigma_{u u}^{4} .
\end{aligned}
$$

Analogamente, temos

(16) $E\left(\bar{u}_{t .}^{2}\right)^{3}=15 r_{t}^{-3} \sigma_{u u}^{3}$.

\section{C.1.2 Cálculo de $\mathbf{E}\left(\mathbf{R}_{3 \mathrm{t}}^{4}\right)$.}

$$
\begin{aligned}
E\left(R_{3 t}^{4}\right)= & \left.E\left\{\left(\sum_{i=1}^{r_{t}}\left(X_{t j}-\bar{X}_{t .}\right)^{2}\right)\right)^{4}\right\}=E\left\{\left(\sum_{i=1}^{r_{t}} u_{t j}^{2}-r_{t} \bar{u}_{t .}^{2}\right)^{4}\right\} \\
= & E\left\{\left(\sum_{i=1}^{r_{t}} u_{t j}^{2}-r_{t}^{-1}\left(\sum_{i=1}^{r_{t}} u_{t j}\right)^{2}\right)^{4}\right\} \\
= & E\left(\sum_{j=1}^{r_{t}} u_{t_{j}}^{2}\right)^{4}+r_{t}^{4} E\left(\bar{u}_{t .}^{8}\right)-4 r_{t} \cdot E\left[\left(\sum_{j=1}^{r_{t}} u_{t_{j}}^{2}\right)^{3}\left(\bar{u}_{t .}^{2}\right)\right] \\
& +6 r_{t}^{2} \cdot E\left[\left(\sum_{j=1}^{r_{t}} u_{t_{j}}^{2}\right)^{2}\left(\bar{u}_{t .}^{4}\right)\right]-4 r_{t}^{3} \cdot E\left[\left(\sum_{j=1}^{r_{t}} u_{t_{j}}^{2}\right)\left(\bar{u}_{t .}^{6}\right)\right] .
\end{aligned}
$$


Substituindo (C.30) e (C.20), em (C.34), temos

$$
\begin{aligned}
E\left(R_{3 t}^{4}\right) & =\left(1-4 r_{t}^{-1}+18 r_{t}^{-2}\right) E\left(\sum_{j=1}^{r_{t}} u_{t_{j}}^{2}\right)^{4}+r_{t}^{4} E\left(\bar{u}_{t .}^{8}\right) \\
& -4 r_{t}^{-3} \cdot E\left[\left(\sum_{j=1}^{r_{t}} u_{t_{j}}^{2}\right)\left(\sum_{j=1}^{r_{t}} u_{t_{j}}\right)^{6}\right]-12 r_{t}^{-2} E\left[\left(\sum_{j=1}^{r_{t}} u_{t_{j}}^{2}\right)^{2}\left(\sum_{j=1}^{r_{t}} u_{t_{j}}^{4}\right)\right] .
\end{aligned}
$$

Substituindo os valores obtidos (C.7), (C.32), (C.21) e (C.27) em (C.35), temos

$$
\begin{aligned}
E\left(R_{3 t}^{4}\right)= & \left\{( 1 - 4 r _ { t } ^ { - 1 } + 1 8 r _ { t } ^ { - 2 } ) \left[105 r_{t}+87 r_{t}\left(r_{t}-1\right) I_{r_{t}}(2)\right.\right. \\
& \left.+18 r_{t}\left(r_{t}-1\right)\left(r_{t}-2\right) I_{r_{t}}(3)+r_{t}\left(r_{t}-1\right)\left(r_{t}-2\right)\left(r_{t}-3\right) I_{r_{t}}(4)\right]+105 \sigma_{u u}^{4} \\
& -4 r_{t}^{-3}\left[105 r_{t}+15 r_{t}\left(r_{t}-1\right)\left(3 r_{t}+19\right) I_{r_{t}}(2)+135 r_{t}\left(r_{t}-1\right) r_{t}-2\right) I_{r_{t}}(3) \\
& \left.+15 r_{t}\left(r_{t}-1\right)\left(r_{t}-2\right)\left(r_{t}-3\right) I_{r_{t}}(4)\right] \\
& \left.-12 r_{t}^{-2}\left[105 r_{t}+3 r_{t}\left(r_{t}-1\right)\left(r_{t}+11\right) I_{r_{t}}(2)\right]\right\} \sigma_{u u}^{4} .
\end{aligned}
$$

Simplificando a expressão (C.36), chegamos, finalmente, a

$$
\begin{aligned}
E\left(R_{3 t}^{4}\right)= & \left\{105\left(r_{t}-3+6 r_{t}^{-1}-4 r_{t}^{-2}\right)+3\left(r_{t}-1\right)\left(29 r_{t}-128+330 r_{t}^{-1}-380 r_{t}^{-2}\right)\right. \\
& \times I_{r_{t}}(2)+18\left(r_{t}-1\right)\left(r_{t}-2\right)\left(r_{t}-4+18 r_{t}^{-1}-30 r_{t}^{-2}\right) I_{r_{t}}(3) \\
& \left.+\left(r_{t}-1\right)\left(r_{t}-2\right)\left(r_{t}-3\right)\left(r_{t}-4+18 r_{t}^{-1}-60 r_{t}^{-2}\right) I_{r_{t}}(4)\right\} \sigma_{u u}^{4} .
\end{aligned}
$$

\section{C.1.3 Cálculo de $\mathrm{E}\left(\mathbf{R}_{3 \mathrm{t}}^{3}\right)$.}

$$
E\left(R_{3 t}^{3}\right)=E\left\{\left(\sum_{i=1}^{r_{t}} u_{t j}^{2}-r_{t} \bar{u}_{t .}^{2}\right)^{3}\right\}=E\left\{\left(\sum_{i=1}^{r_{t}} u_{t j}^{2}-r_{t}^{-1}\left(\sum_{i=1}^{r_{t}} u_{t j}\right)^{2}\right)^{3}\right\}
$$




$$
\begin{aligned}
= & E\left(\sum_{j=1}^{r_{t}} u_{t_{j}}^{2}\right)^{3}-r_{t}^{3} E\left(\bar{u}_{t_{.}}^{6}\right)-3 r_{t}^{-1} \cdot E\left[\left(\sum_{j=1}^{r_{t}} u_{t_{j}}^{2}\right)^{2}\left(\sum_{j=1}^{r_{t}} u_{t_{j}}\right)^{2}\right] \\
& +3 r_{t}^{-2} E\left[\left(\sum_{j=1}^{r_{t}} u_{t_{j}}^{2}\right)\left(\sum_{j=1}^{r_{t}} u_{t_{j}}\right)^{4}\right] .
\end{aligned}
$$

Mas,

$$
E\left[\left(\sum_{j=1}^{r_{t}} u_{t_{j}}^{2}\right)\left(\sum_{j=1}^{r_{t}} u_{t_{j}}\right)^{4}\right]=E\left[\left(\sum_{j=1}^{r_{t}} u_{t_{j}}^{2}\right)\left(\sum_{j=1}^{r_{t}} u_{t_{j}}^{4}+6 \sum_{j=1}^{r_{t}-1} \sum_{i>j}^{r_{t}} u_{t_{j}}^{2} \cdot u_{t_{i}}^{2}\right)\right]
$$

e

$$
\left(\sum_{j=1}^{r_{t}} u_{t_{j}}^{2}\right)^{2}=\left(\sum_{j=1}^{r_{t}} u_{t_{j}}^{4}\right)+2 I_{r_{t}}(2)\left(\sum_{j=1}^{r_{t}-1} \sum_{i>j}^{r_{t}} u_{t_{j}}^{2} u_{t_{i}}^{2}\right) .
$$

De (C.40), temos

$$
\left(\sum_{j=1}^{r_{t}} u_{t_{j}}^{4}\right)+6 I_{r_{t}}(2)\left(\sum_{j=1}^{r_{t}-1} \sum_{i>j}^{r_{t}} u_{t_{j}}^{2} \cdot u_{t_{i}}^{2}\right)=\left(\sum_{j=1}^{r_{t}} u_{t_{j}}^{2}\right)^{2}+4 I_{r_{t}}(2)\left(\sum_{j=1}^{r_{t}-1} \sum_{i>j}^{r_{t}} u_{t_{j}}^{2} \cdot u_{t_{i}}^{2}\right) .
$$

Substituindo (C.41) em (C.39), temos

$$
E\left[\left(\sum_{j=1}^{r_{t}} u_{t_{j}}^{2}\right)\left(\sum_{j=1}^{r_{t}} u_{t_{j}}\right)^{4}\right]=E\left(\sum_{j=1}^{r_{t}} u_{t_{j}}^{2}\right)^{3}+4 I_{r_{t}}(2) E\left[\left(\sum_{j=1}^{r_{t}} u_{t_{j}}^{2}\right)\left(\sum_{j=1}^{r_{t}-1} \sum_{i>j}^{r_{t}} u_{t_{j}}^{2} \cdot u_{t_{i}}^{2}\right)\right] .
$$

Temos, também, que

$$
\begin{aligned}
E\left[\left(\sum_{j=1}^{r_{t}} u_{t_{j}}^{2}\right)^{2}\left(\sum_{j=1}^{r_{t}} u_{t_{j}}\right)^{2}\right] & =E\left[\left(\sum_{j=1}^{r_{t}} u_{t_{j}}\right)^{2}\left(\sum_{j=1}^{r_{t}} u_{t_{j}}^{2}+2 \sum_{j=1}^{r_{t}-1} \sum_{i>j}^{r_{t}} u_{t_{j}} u_{t_{i}}\right)\right] \\
& =E\left[\left(\sum_{j=1}^{r_{t}} u_{t_{j}}^{2}\right)^{3}\right] .
\end{aligned}
$$


Agora, substituindo (C.42) e (C.43) em (C.38), temos

$$
\begin{aligned}
E\left(R_{3 t}^{3}\right)= & \left(1-3 r_{t}^{-1}+3 r_{t}^{-2}\right) E\left(\sum_{i=1}^{r_{t}} u_{t j}^{2}\right)^{3}+3 r_{t}^{-2}\left\{4 E\left[\left(\sum_{i=1}^{r_{t}} u_{t j}^{2}\right)\left(\sum_{j=1}^{r_{t}-1} \sum_{i>j}^{r_{t}} u_{t_{j}}^{2} \cdot u_{t_{i}}^{2}\right)\right]\right\} \\
& -r_{t}^{3} E\left(\bar{u}_{t .}^{6}\right) .
\end{aligned}
$$

Finalmente, substituindo (C.10), (C.13) e (C.33) em (C.44), obtemos

$$
\begin{aligned}
E\left(R_{3 t}^{3}\right)= & \left\{\left(1-3 r_{t}^{-1}+3 r_{t}^{-2}\right)\left[15 r_{t}+9 r_{t}\left(r_{t}-1\right) I_{r_{t}}(2)+r_{t}\left(r_{t}-1\right)\left(r_{t}-2\right) I_{r_{t}}(3)\right]\right. \\
& \left.+12 r_{t}^{-2}\left[\frac{1}{2} r_{t}\left(r_{t}-1\right)\left(r_{t}+4\right)\right]-r_{t}^{3} r_{t}^{-3} 15\right\} \sigma_{u u}^{3},
\end{aligned}
$$

que simplificada resulta

$$
\begin{aligned}
E\left(R_{3 t}^{3}\right)= & \left\{15\left(r_{t}-4+3 r_{t}^{-1}\right)+3\left(r_{t}-1\right)\left(3 r_{t}-7+17 r_{t}^{-1}\right) I_{r_{t}}(2)+\right. \\
& \left.+\left(r_{t}-1\right)\left(r_{t}-2\right)\left(r_{t}-3+3 r_{t}^{-1}\right) I_{r_{t}}(3)\right\} \sigma_{u u}^{3} .
\end{aligned}
$$

\section{C.2 Obtenção de cotas superiores de $E\left(R_{33 t}^{i}\right)$;}

$$
i=1, \ldots, 4 \text {. }
$$

As cotas obtidas nesta seção serão empregadas na seção seguinte.

Notação utilizada:

$$
R_{33 t}=a_{33} R_{3 t}-A,
$$

onde

$$
\begin{gathered}
a_{33}=a_{3} \nu_{n} ; \nu_{n}=\frac{\sum_{t=1}^{n} \frac{1}{r_{t}}}{\sum_{t=1}^{n}\left(r_{t}-1\right)} ; A=a_{1} \sigma_{\bar{X} \bar{X} t}+a_{2} \sigma_{\bar{X} Y}+a_{33} E\left(R_{3 t}\right) \quad \text { e } \\
R_{3 t}= \begin{cases}\sim \sigma_{u u} \chi^{2}\left(r_{t}-1\right), & \text { para } r_{t}>1 \\
0, & \text { para } r_{t}=1 .\end{cases}
\end{gathered}
$$


Resultados já obtidos:

$$
\begin{aligned}
E\left(R_{3 t}\right)= & \left(r_{t}-1\right) \sigma_{u u} ; \\
\operatorname{Var}\left(R_{3 t}\right)= & 2\left(r_{t}-1\right) \sigma_{u u}^{2}=\left(\frac{2}{r_{t}-1}\right)\left[E\left(R_{3 t}\right)\right]^{2} ; \\
E\left(R_{3 t}^{2}\right)= & \left(r_{t}^{2}-1\right) \sigma_{u u}^{2}=\left(r_{t}+1\right)\left(r_{t}-1\right) \sigma_{u u}^{2}=\frac{\left(r_{t}+1\right)}{\left(r_{t}-1\right)}\left[E\left(R_{3 t}\right)\right]^{2} ; \\
E\left(R_{3 t}^{3}\right)= & \left\{15\left(r_{t}-4+3 r_{t}-1\right)+3\left(r_{t}-1\right)\left(3 r_{t}-7+17 r_{t}^{-1}\right) I_{r_{t}}(2)\right. \\
& \left.+\left(r_{t}-1\right)\left(r_{t}-2\right)\left(r_{t}-3+3 r_{t}^{-1}\right) I_{r_{t}}(3)\right\} \sigma_{u u}^{3} \\
= & Q_{1}\left(r_{t}\right) \sigma_{u u}^{3} ;
\end{aligned}
$$

e

$$
\begin{aligned}
E\left(R_{3 t}^{4}\right)= & \left\{105\left(r_{t}-3+6 r_{t}^{-1}-4 r_{t}^{-2}\right)+3\left(r_{t}-1\right)\left(29 r_{t}-128+330 r_{t}^{-1}-380 r_{t}^{-2}\right) \times\right. \\
& \times I_{r_{t}}(2)+18\left(r_{t}-1\right)\left(r_{t}-2\right)\left(r_{t}-4+18 r_{t}^{-1}-30 r_{t}^{-2}\right) I_{r_{t}}(3) \\
& \left.+\left(r_{t}-1\right)\left(r_{t}-2\right)\left(r_{t}-3\right)\left(r_{t}-4+18 r_{t}^{-1}-60 r_{t}^{-2}\right) I_{r_{t}}(4)\right\} \sigma_{u u}^{4} \\
= & Q_{2}\left(r_{t}\right) \sigma_{u u}^{4} .
\end{aligned}
$$

Temos, ainda que

$$
\begin{aligned}
E\left(R_{33 t}\right)= & a_{33} E\left(R_{3 t}\right)-A=-\left(a_{1} \sigma_{\bar{X} \bar{X} t}+a_{2} \sigma_{\bar{X} Y}\right), \\
R_{33 t}= & a_{33} R_{3 t}-A=a_{33} R_{3 t}-a_{33} E\left(R_{3 t}\right)-\left(a_{1} \sigma_{\bar{X} \bar{X} t}+a_{2} \sigma_{\bar{X} Y}\right) \\
& =a_{33}\left(R_{3 t}-E\left(R_{3 t}\right)\right)+E\left(R_{33 t}\right), \\
\operatorname{Var}\left(R_{33 t}\right)= & a_{33}^{2} \operatorname{Var}\left(R_{3 t}\right)=a_{33}^{2}\left[2\left(r_{t}-1\right) \sigma_{u u}^{2}\right],
\end{aligned}
$$

$\mathrm{e}$

$$
\begin{aligned}
E\left(R_{33 t}^{2}\right) & =\operatorname{Var}\left(R_{33 t}\right)+\left[E\left(R_{33 t}\right)\right]^{2}=\frac{2 a_{33}^{2}}{\left(r_{t}-1\right)}\left[E\left(R_{3 t}\right)\right]^{2}+\left[E\left(R_{33 t}\right)\right]^{2} \\
& <a_{33}^{2}\left[2\left(r_{t}+1\right) E\left(R_{3 t}\right)\right]^{2}+\left[E\left(R_{33 t}\right)\right]^{2}
\end{aligned}
$$

(a) Obtenção de cotas para $E\left(R_{33 t}^{3}\right)$ e $E\left(R_{33 t}^{4}\right)$ 
Temos, inicialmente, que

$$
\begin{gathered}
E\left(R_{3 t}^{3}\right)=\frac{Q_{1}\left(r_{t}\right)}{\left(r_{t}-1\right)^{3}}\left[\left(r_{t}-1\right) \sigma_{u u}\right]^{2}=\frac{Q_{1}\left(r_{t}\right)}{\left(r_{t}-1\right)^{3}}\left[E\left(R_{3 t}\right)\right]^{3} \\
E\left(R_{3 t}^{4}\right)=\frac{Q_{2}\left(r_{t}\right)}{\left(r_{t}-1\right)^{4}}\left[E\left(R_{3 t}\right)\right]^{4} .
\end{gathered}
$$

Mas,

$$
\begin{aligned}
Q_{1}\left(r_{t}\right)= & 15\left(r_{t}-4+3 r_{t}^{-1}\right)+3\left(r_{t}-1\right)\left(3 r_{t}-7+17 r_{t}^{-1}\right) I_{r_{t}}(2) \\
& +\left(r_{t}-1\right)\left(r_{t}-2\right)\left(r_{t}-3+3 r_{t}^{-1}\right) I_{r_{t}}(3)
\end{aligned}
$$

Agora, para $r_{t} \geq 2$, temos

$$
3 r_{t}-7+17 r_{t}^{-1}<\frac{3 r_{t}-6+18 r_{t}^{-1}}{3}=\left(r_{t}-2+3 r_{t}^{-1}\right)>\left(r_{t}-4+3 r_{t}^{-1}\right)
$$

Substituindo as desigualdades acima (C.55) em (C.54), temos

$$
Q_{1}\left(r_{t}\right)<\left(r_{t}-2+3 r_{t}^{-1}\right)\left[15+9\left(r_{t}+1\right) I_{r_{t}}(2)+\left(r_{t}-1\right)\left(r_{t}-2\right) I_{r_{t}}(3)\right] .
$$

Como, para $r_{t} \geq 2,\left(r_{t}-1\right)>\left(r_{t}-3+3 r_{t}^{-1}\right)$, temos que

$$
\begin{aligned}
Q_{1}\left(r_{t}\right) & <\left(r_{t}-1\right)\left[9\left(3 r_{t}-1\right)+15+\left(r_{t}-1\right)\left(r_{t}-2\right) I_{r_{t}}(3)\right] \\
& =\left(r_{t}-1\right)\left[9 r_{t}+6+\left(r_{t}^{2}-3 r_{t}+2\right) I_{r_{t}}(3)\right] \\
& <\left(r_{t}-1\right)\left[6 r_{t}+6+\left(r_{t}^{2}+2\right)\right] \\
& <\left(r_{t}-1\right)\left[6\left(r_{t}+1\right)+\left(r_{t}+1\right)^{2}\right] \\
& <\left(r_{t}-1\right)\left[6\left(r_{t}+1\right)^{2}+\left(r_{t}+1\right)^{2}\right] \\
& =7\left(r_{t}-1\right)\left(r_{t}+1\right)^{2}<\left[2\left(r_{t}+1\right)\right]^{3}
\end{aligned}
$$

Por outro lado, temos

$$
\begin{aligned}
Q_{2}\left(r_{t}\right)= & 105\left(r_{t}-3+6 r_{t}^{-1}-4 r_{t}^{-2}\right) \\
& +3\left(r_{t}-1\right)\left(29 r_{t}-128+330 r_{t}^{-1}-380 r_{t}^{-2}\right) I_{r_{t}}(2) \\
& +18\left(r_{t}-1\right)\left(r_{t}-2\right)\left(r_{t}-4+18 r_{t}^{-1}-30 r_{t}^{-2}\right) I_{r_{t}}(3) \\
& +\left(r_{t}-1\right)\left(r_{t}-2\right)\left(r_{t}-3\right)\left(r_{t}-4+18 r_{t}^{-1}-60 r_{t}^{-2}\right) I_{r_{t}}(4) .
\end{aligned}
$$


Mas, para $r_{t} \geq 2$, temos

$$
r_{t}-3+9 r_{t}^{-1}-4 r_{t}^{-2}>\left\{\begin{array}{l}
r_{t}-3+6 r_{t}^{-1}-4 r_{t}^{-2}, \\
r_{t}-\frac{128}{29}+\frac{330}{29} r_{t}^{-1}-\frac{380}{29} r_{t}^{-2}, \\
r_{t}-4+18 r_{t}^{-1}-30 r_{t}^{-2} .
\end{array}\right.
$$

Substituindo as desigualdades (C.59) em (C.58), temos

$$
\begin{aligned}
Q_{2}\left(r_{t}\right)< & \left\{( r _ { t } - 3 + 9 r _ { t } ^ { - 1 } - 4 r _ { t } ^ { - 2 } ) \left[105+87\left(r_{t}-1\right) I_{r_{t}}\right.\right. \\
+ & \left.\left.18\left(r_{t}-1\right)\left(r_{t}-2\right) I_{r_{t}}(3)+\left(r_{t}-1\right)\left(r_{t}-2\right)\left(r_{t}-3\right) I_{r_{t}}(4)\right]\right\} .
\end{aligned}
$$

Como

$$
r_{t}-3+9 r_{t}^{-1}+4 r_{t}^{-2}<2\left(r_{t}-1\right),
$$

substituindo (C.61) em (C.60), temos

$$
\begin{aligned}
Q_{2}\left(r_{t}\right) & <2\left(r_{t}-1\right)^{2}\left[105+87 I_{r_{t}}(2)+18\left(r_{t}-2\right) I_{r_{t}}(3)+\left(r_{t}-2\right)\left(r_{t}-3\right) I_{r_{t}}(4)\right] \\
& <\left[2\left(r_{t}-1\right)^{4}(105+87+18+1)\right] \\
& =2\left(r_{t}-1\right)^{4}(211)=422\left(r_{t}-1\right)^{4} .
\end{aligned}
$$

Temos, ainda, que

$$
E\left(R_{3 t}^{4}\right)=Q_{2}\left(r_{t}\right) \sigma_{u u}^{4}=\frac{Q_{2}\left(r_{t}\right)}{\left(r_{t}-1\right)^{4}}\left[\left(r_{t}-1\right) \sigma_{u u}\right]^{4}=\frac{Q_{2}\left(r_{t}\right)}{\left(r_{t}-1\right)^{4}}\left[E\left(R_{3 t}\right)\right]^{4} .
$$

Substituindo, a desigualdade (C.62) em (C.63), temos:

$$
E\left(R_{3 t}^{4}\right)<\frac{422\left(r_{t}-1\right)^{4}}{\left(r_{t}-1\right)^{4}}\left[E\left(R_{3 t}\right)\right]^{4}=(422)\left[E\left(R_{3 t}\right)^{4}\right]
$$

e como para, $r_{t} \geq 2$, temos que

$$
\left(r_{t}+1\right) \geq 3 \Longrightarrow 2\left(r_{t}+1\right) \geq 6 \Longrightarrow\left[2\left(r_{t}+1\right)\right]^{4} \geq 1296 .
$$

De (C.65), temos

$$
E\left(R_{3 t}^{4}\right)<\left[2\left(r_{t}+1\right)\right]^{4}\left[E\left(R_{3 t}\right)\right]^{4}=\left[2\left(r_{t}+1\right) E\left(R_{3 t}\right)\right]^{4} .
$$


Finalmente, temos as cotas de $E\left(R_{3 t}^{i}\right), \quad i=1, \cdots, 4$, em função de $E\left(R_{3 t}\right)$

$$
\begin{aligned}
& E\left(R_{3 t}\right)=\left(r_{t}-1\right) \sigma_{u u}<2\left(r_{t}+1\right) \sigma_{u u} \\
& E\left(R_{3 t}^{2}\right)<\left[2\left(r_{t}+1\right) E\left(R_{3 t}\right)\right]^{2} \\
& E\left(R_{3 t}^{3}\right)<\left[2\left(r_{t}+1\right) E\left(R_{3 t}\right)\right]^{3} \\
& E\left(R_{3 t}^{4}\right)<\left[2\left(r_{t}+1\right) E\left(R_{3 t}\right)\right]^{4}
\end{aligned}
$$

(b) Obtenção das cotas superiores para $E\left(R_{33 t}^{i}\right), \quad i=1,2,3,4$.

De (C.49) a (C.52), temos

$$
\begin{aligned}
E\left(R_{33 t}\right) & =-\left(a_{1} \sigma_{\bar{X} \bar{X} t}+a_{2} \sigma_{\bar{X} Y}\right) \leq\left|a_{1} \sigma_{\bar{X} \bar{X} t}+a_{2} \sigma_{\bar{X} Y}\right| \\
\operatorname{Var}\left(R_{33 t}\right) & =a_{33}^{2}\left[2\left(r_{t}-1\right) \sigma_{u u}^{2}\right] \\
E\left(R_{33 t}^{2}\right) & <a_{33}^{2}\left[2\left(r_{t}+1\right) E\left(R_{3 t}\right)\right]^{2}+\left[E\left(R_{33 t}^{3}\right)\right]^{2} .
\end{aligned}
$$

Note, também, que

$$
\begin{aligned}
E\left(R_{33 t}^{3}\right)= & E\left\{\left[a_{33} R_{3 t}-E\left(R_{3 t}\right)+E\left(R_{33 t}\right)\right]^{3}\right\} \\
= & a_{33}^{3} E\left[R_{3 t}-E\left(R_{3 t}\right)\right]^{3}+\left[E\left(R_{33 t}\right)\right]^{3}+3 a_{33}^{2}\left\{E\left[R_{3 t}-E\left(R_{3 t}\right)\right]^{2}\right\} \\
& \times\left[E\left(R_{33 t}\right)\right]+3 a_{33} E\left[R_{3 t}-E\left(R_{3 t}\right)\right]\left[E\left(R_{33 t}\right)\right]^{2} \\
= & a_{33}^{3} E\left[R_{3 t}-E\left(R_{3 t}\right)\right]^{3}+\left[E\left(R_{33 t}\right)\right]^{3}+3 a_{33}^{2} \operatorname{Var}\left(R_{3 t}\right)\left[E\left(R_{33 t}\right)\right] .
\end{aligned}
$$

Mas

$$
\begin{aligned}
E\left[R_{3 t}-E\left(R_{3 t}\right)\right]^{3} & =E\left\{R_{3 t}^{3}-\left[E\left(R_{3 t}\right)\right]^{3}-3 R_{3 t}^{2}\left(E\left(R_{3 t}\right)\right)+3 R_{3 t}\left(-E\left(R_{3 t}\right)\right)^{2}\right\} \\
& =E\left(R_{3 t}^{3}\right)-\left[E\left(R_{3 t}\right)\right]^{3}+3\left[E\left(R_{3 t}\right)\right]^{3}-3\left[E\left(R_{3 t}\right)\right]\left[E\left(R_{3 t}^{2}\right)\right] \\
& =E\left(R_{3 t}^{3}\right)+2\left[E\left(R_{3 t}\right)\right]^{3}-3\left[E\left(R_{3 t}\right)\right]\left(\frac{r_{t}+1}{r_{t}-1}\right)\left[E\left(R_{3 t}\right)\right]^{2} \\
& =E\left(R_{3 t}^{3}\right)-\frac{\left(r_{t}+4\right)}{\left(r_{t}-1\right)}\left[E\left(R_{3 t}\right)\right]^{3} \\
& <E\left(R_{3 t}^{3}\right) .
\end{aligned}
$$


Substituindo (C.70) em (C.69), obtemos

$$
\begin{aligned}
E\left(R_{33 t}^{3}\right) & =a_{33}^{3}\left\{E\left(R_{3 t}^{3}\right)-\frac{\left(r_{t}+4\right)}{\left(r_{t}-1\right)}\left[E\left(R_{3 t}\right)\right]^{3}\right\}+\left[E\left(R_{33 t}\right)\right]^{3}+3 a_{33}^{2} \operatorname{Var}\left(R_{3 t}\right)\left[E\left(R_{33 t}\right)\right] \\
& <a_{33}^{3} E\left(R_{3 t}^{3}\right)+\left[E\left(R_{33 t}\right)\right]^{3}+3 a_{33}^{2}\left\{2\left(r_{t}+1\right) E\left(R_{3 t}\right)\right\}^{2} \\
& <\left|a_{33}\right|^{3}\left[2\left(r_{t}+1\right) E\left(R_{3 t}\right)\right]^{3}+\left[E\left(R_{33 t}\right)\right]^{3}+3 a_{33}^{2}\left\{2\left(r_{t}+1\right) E\left(R_{3 t}\right\}^{2}\right. \\
& \leq\left\{\left|a_{33}\right| 2\left(r_{t}+1\right) E\left(R_{3 t}\right)+\left|E\left(R_{33 t}\right)\right|\right\}^{3} .
\end{aligned}
$$

Por outro lado, temos, também, que

$$
\begin{aligned}
E\left[R_{3 t}-E\left(R_{3 t}\right)\right]^{4} & =E\left(R_{3 t}^{4}\right)-4\left[E\left(R_{3 t}^{3}\right)\right]\left[E\left(R_{3 t}\right)\right]+6 E\left(R_{3 t}^{2}\right)\left[E\left(R_{3 t}\right)\right]^{2}-3\left[E\left(R_{3 t}\right)\right]^{4} \\
& =E\left(R_{3 t}^{4}\right)+Q_{4}\left(r_{t}\right) .
\end{aligned}
$$

Também,

$$
\begin{aligned}
6 E\left(R_{3 t}^{2}\right)\left[E\left(R_{3 t}\right)\right]^{2}-3\left[E\left(R_{3 t}\right)\right]^{4} & =\left[E\left(R_{3 t}\right)\right]^{4}\left\{\frac{6\left(r_{t}+1\right)}{r_{t}-1}-3\right\} \\
& =\frac{3\left(r_{t}+3\right)}{\left(r_{t}-1\right)}\left[E\left(R_{3 t}\right)\right]^{4} .
\end{aligned}
$$

De (C.73) e (C.47) temos

$$
\begin{aligned}
Q_{4}\left(r_{t}\right) & =-\frac{\left[E\left(R_{3 t}\right)\right]^{4}}{\left(r_{t}-1\right)^{3}}\left[4 Q_{1}\left(r_{t}\right)-3\left(r_{t}+3\right)\left(r_{t}-1\right)^{2}\right] \\
& =-\frac{\left[E\left(R_{3 t}\right)\right]^{4}}{\left(r_{t}-1\right)^{3}} Q_{3}\left(r_{t}\right) .
\end{aligned}
$$

De (C.47), podemos escrever

$$
\begin{aligned}
Q_{3}\left(r_{t}\right)= & 4 Q_{1}\left(r_{t}\right)-3\left(r_{t}+3\right)\left(r_{t}-1\right)^{2} \\
= & {\left[60\left(r_{t}-4+3 r_{t}^{-1}\right)+3\left(r_{t}-1\right)\left(12 r_{t}-28+68 r_{t}^{-1}\right) I_{r_{t}}(2)\right.} \\
& \left.+4\left(r_{t}-1\right)\left(r_{t}-2\right)\left(r_{t}-3+3 r_{t}^{-1}\right) I_{r_{t}}(3)\right]-3\left(r_{t}+3\right)\left(r_{t}-1\right)^{2} \\
= & 60\left(r_{t}-4+3 r_{t}^{-1}\right)+3\left(r_{t}-1\right)\left[12 r_{t}-28+68 r_{t}^{-1}-\left(r_{t}-1\right)\left(r_{t}+3\right)\right] I_{r_{t}}(2) \\
& +4\left(r_{t}-1\right)\left(r_{t}-2\right)\left(r_{t}-3+3 r_{t}^{-1}\right) I_{r_{t}}(3) .
\end{aligned}
$$


Mas

$$
3\left(r_{t}-1\right)\left[12 r_{t}-28+68 r_{t}^{-1}-\left(r_{t}^{2}+2 r_{t}-3\right)\right]=3\left(r_{t}-1\right)\left(-r_{t}^{2}+10 r_{t}-25+68 r_{t}^{-1}\right) .
$$

Substituindo (C.76) em (C.75), obtemos

$$
\begin{aligned}
Q_{3}\left(r_{t}\right)= & {\left[3\left(r_{t}-1\right)\left(-r_{t}^{2}+10 r_{t}-25+68 r_{t}^{-1}\right)+60\left(r_{t}-4+3 r_{t}^{-1}\right)\right] I_{r_{t}}(2) } \\
& +4\left(r_{t}-1\right)\left(r_{t}-2\right)\left(r_{t}-3+3 r_{t}^{-1}\right) I_{r_{t}}(3) .
\end{aligned}
$$

De (C.77), vemos que se $r_{t}=2$,

$$
Q_{3}(2)=3(-4+20-25+34)+60\left(2-4+\frac{3}{2}\right)=3(25)-30=45 .
$$

Para $r_{t} \geq 3$ temos

$$
Q_{3}\left(r_{t}\right)=\left(r_{t}-1\right)\left(r_{t}^{2}+10 r_{t}-39+180 r_{t}^{-1}\right)+60\left(r_{t}-4+3 r_{t}^{-1}\right) .
$$

Portanto, para $r_{t} \geq 2, Q_{3}\left(r_{t}\right)>0$, e, consequentemente, de (C.74), $Q_{4}\left(r_{t}\right)<0$. De (C.72), podemos, então, concluir que

$$
E\left[R_{3 t}-E\left(R_{3 t}\right)\right]^{4}<E\left(R_{3 t}\right)^{4} .
$$

Por outro lado, de (C.78) e (C.70), temos

$$
\begin{aligned}
E\left(R_{33 t}\right)^{4}= & \sum_{i=0}^{4}\left(\begin{array}{l}
4 \\
i
\end{array}\right) E\left\{\left[a_{33} R_{3 t}-E\left(R_{3 t}\right)\right]\right\}^{4-i}\left[E\left(R_{33 t}\right)\right]^{i} \\
= & a_{33}^{4} E\left\{\left[R_{3 t}-E\left(R_{3 t}\right)\right]^{4}\right\}+4 a_{33}^{3} E\left\{\left[R_{3 t}-E\left(R_{3 t}\right)\right]^{3}\right\}\left[E\left(R_{33 t}\right)\right] \\
& +6 a_{33}^{2} E\left\{\left[R_{3 t}-E\left(R_{3 t}\right)\right]^{2}\right\}\left[E\left(R_{33 t}\right)\right]^{2}+\left[E\left(R_{33 t}\right)\right]^{4} \\
< & a_{33}^{4} E\left(R_{3 t}\right)^{4}+4\left|a_{33}\right|^{3} E\left(R_{3 t}^{3}\right)\left[E\left(R_{33 t}\right)\right] \\
& +6 a_{33}^{2}\left[\operatorname{Var}\left(R_{3 t}\right)\right]\left[E\left(R_{33 t}\right)\right]^{2}+\left[E\left(R_{33 t}\right)\right]^{4}
\end{aligned}
$$

Agora de (C.67), temos que

$$
\begin{aligned}
E\left(R_{33 t}\right)^{4}< & a_{33}^{4}\left[2\left(r_{t}+1\right) E\left(R_{3 t}\right)\right]^{4}+4\left|a_{33}\right|^{3}\left[2\left(r_{t}+1\right) E\left(R_{3 t}\right)\right]^{3} E\left(R_{33 t}\right) \\
& +6 a_{33}\left[2\left(r_{t}+1\right) E\left(R_{3 t}\right)\right]^{2}\left[E\left(R_{33 t}\right)\right]^{2}+\left[E\left(R_{33 t}\right)\right]^{4} \\
\leq & {\left[\left|a_{33}\right|\left[2\left(r_{t}+1\right) E\left(R_{3 t}\right)\right]+\left[E\left(R_{33 t}\right)\right]^{4}\right.}
\end{aligned}
$$


Resumindo os resultados obtidos

$$
\begin{aligned}
& E\left(R_{33 t}\right) \leq\left|a_{1} \sigma_{\bar{X} \bar{X} t}+a_{2} \sigma_{\bar{X} Y}\right| \\
& E\left(R_{33 t}^{2}\right)<\left\{\left|a_{33}\right|\left[2\left(r_{t}+1\right) E\left(R_{3 t}\right)\right]+\left|E\left(R_{33 t}\right)\right|\right\}^{2} ; \\
& E\left(R_{33 t}^{3}\right)<\left\{\left|a_{33}\right|\left[2\left(r_{t}+1\right) E\left(R_{3 t}\right)\right]+\left|E\left(R_{33 t}\right)\right|\right\}^{3} ; \\
& E\left(R_{33 t}^{4}\right)<\left\{\left|a_{33}\right|\left[2\left(r_{t}+1\right) E\left(R_{3 t}\right)\right]+\left|E\left(R_{33 t}\right)\right|\right\}^{4} .
\end{aligned}
$$

\section{C.3 Prova que $E\left\{\left(U_{i t}\right)^{4}\right\}<\infty, i=1,2,3$.}

Apresentamos os cálculos concernentes à prova de que $E\left\{\left(U_{3 t}\right)^{4}\right\}<\infty$; para as provas das outras duas variáveis os cálculos são análogos, portanto, só apresentamos os resultados finais delas no final desta Seção.

Temos que

$$
\begin{aligned}
U_{3 t}= & a_{1}\left[\left(\bar{X}_{t .}-\mu_{x}\right)^{2}-\sigma_{\bar{X} \bar{X} t}\right]+a_{2}\left[\left(\bar{X}_{t .}-\mu_{x}\right)\left(Y_{t}-\mu_{y}\right)-\sigma_{\bar{X} Y}\right] \\
& +a_{3} \nu_{n}\left[R_{3 t}-\left(r_{t}-1\right) \sigma_{u u}\right]
\end{aligned}
$$

com

$$
R_{3 t}=\sum_{j=1}^{r_{t}-1}\left(u_{t j}-\bar{u}_{t}\right)^{2}, \quad\left(a_{1}, a_{2}, a_{3}\right) \in \mathbf{R}^{3} \quad \text { e } \quad \nu_{n}=\frac{\sum_{t=1}^{n} \frac{1}{r_{t}}}{\sum_{t=1}^{n}\left(r_{t}-1\right)} .
$$

A expressão (C.82) pode ser reescrita como

$$
\begin{aligned}
U_{3 t}= & {\left[\left(x_{t}+\bar{u}_{t .}\right)-\mu_{x}\right]\left\{a_{1}\left[\left(x_{t}+\bar{u}_{t .}\right)-\mu_{x}\right]+a_{2}\left[\beta\left(x_{t}-\mu_{x}\right)+e_{t}\right]\right\} } \\
& +a_{3} \nu_{n} R_{3 t}-A \\
= & {\left[\left(a_{1}+a_{2} \beta\right) \sigma_{x x}\right] Z_{x_{t}}^{2}+\left\{\left[\sqrt{\sigma_{x x}}\left(2 a_{1}+a_{2} \beta\right) \sqrt{\frac{\sigma_{u u}}{r_{t}}}\right] Z_{\bar{u}_{t .}}+a_{2} \sqrt{\sigma_{e e} \sigma_{x x}} Z_{e_{t}}\right\} Z_{x_{t}} } \\
& +\left[a_{1}\left(\frac{\sigma_{u u}}{r_{t}}\right)\right] Z_{\bar{u}_{t .}}^{2}+\left(a_{2} \sqrt{\frac{\sigma_{u u} \sigma_{e e}}{r_{t}}}\right) Z_{\bar{u}_{t .}} Z_{e_{t}}+a_{3} \nu_{n} R_{3 t}-A .
\end{aligned}
$$

Por sua vez, a expressão (C.83) pode ser escrita como:

$$
U_{3 t}=C_{31}+C_{32}+C_{33}
$$


onde

$$
\begin{aligned}
C_{31}= & b_{11} Z_{x_{t}}^{2}, \quad \operatorname{com} Z_{x_{t}}=\frac{x_{t}-\mu_{x}}{\sqrt{\sigma_{x x}}} \text { e } \quad b_{11}=\left(a_{1}+a_{2} \beta\right) \sigma_{x x}, \\
C_{32}= & \left(b_{21} Z_{\bar{u}_{t .}}+b_{22} Z_{e_{t}}\right) Z_{x_{t}}, \quad \text { com } Z_{\bar{u}_{t .}}=\frac{\bar{u} t .}{\sqrt{\frac{\sigma_{u u}}{r_{t}}}}, \quad Z_{e_{t}}=\frac{e_{t}}{\sqrt{\sigma_{e e}}}, \\
& b_{21}=\left(2 a_{1}+a_{2} \beta\right) \sqrt{\frac{\sigma_{u u} \sigma_{x x}}{r_{t}}} \text { e } b_{22}=a_{2} \sqrt{\sigma_{x x} \sigma_{e e}}, \\
C_{33}= & b_{31} Z_{\bar{u}_{t .}}^{2}+b_{32} Z_{\bar{u}_{t .}} Z_{e_{t}}+R_{33 t}, \quad \text { com } \quad b_{31}=a_{1} \frac{\sigma_{u u}}{r_{t}}, \quad b_{32}=a_{2} \sqrt{\frac{\sigma_{u u} \sigma_{e e}}{r_{t}}}, \\
& R_{33 t}=a_{33} R_{3 t}-A, a_{33}=\nu_{n} a_{3} \text { e } A=a_{1} \sigma_{\bar{X} \bar{X} t}+a_{2} \sigma_{\bar{X} Y}+a_{33} E\left(R_{3 t}\right) .
\end{aligned}
$$

De (C.84), e usando o polinômio de Leibniz, temos

$$
\begin{aligned}
E\left(U_{t 3}^{4}\right)= & E\left(C_{31}+C_{32}+C_{33}\right)^{4} \\
= & \sum_{i=1}^{3} E\left(C_{3 i}\right)^{4}+4 \sum_{i>j}^{3} \sum_{j=1}^{2} E\left(C_{3 j}^{3} C_{3 i}+C_{3 j} C_{3 i}^{3}\right)+6 \sum_{i>j}^{3} \sum_{j=1}^{2} E\left(C_{3 j}^{2} C_{3 i}^{2}\right) \\
& +12 E\left(C_{31}^{2} C_{32} C_{33}+C_{31} C_{32}^{2} C_{33}+C_{31} C_{32} C_{33}^{2}\right) .
\end{aligned}
$$

\section{C.3.1 Cálculos preliminares}

I) Cálculo de $\sum_{i=1}^{3} E\left(C_{3 i}\right)^{4}$

Temos:

$$
\sum_{i=1}^{3} E\left(C_{3 i}\right)^{4}=E\left(C_{31}\right)^{4}+E\left(C_{32}\right)^{4}+E\left(C_{33}\right)^{4}
$$

mas,

$$
\begin{aligned}
E\left[\left(C_{31}\right)^{4}\right]= & E\left(b_{11} Z_{x_{t}}^{2}\right)^{4}=105 b_{11}^{4}, \\
E\left[\left(C_{32}\right)^{4}\right]= & E\left\{\left(b_{21} Z_{\bar{u}_{t .}}+b_{22} Z_{e_{t}}\right)^{4}\left(Z_{x_{t}}\right)^{4}\right\} \\
= & E\left(Z_{x_{t}}^{4}\right) E\left\{\left(b_{21}^{4} Z_{\bar{u}_{t .}}^{4}+b_{22}^{4} Z_{e_{t}}^{4}\right)+4\left[\left(b_{21} z_{\bar{u}_{t .}}\right)^{3}\left(b_{22} Z_{e_{t}}\right)+\left(b_{21} Z_{\bar{u}_{t .}}\right)\left(b_{22} Z_{e_{t}}\right)^{3}\right]\right. \\
& +6\left[\left(b_{21} Z_{\bar{u} t .}\right)^{2}\left(b_{22} Z_{e_{t}}\right)^{2}\right]=9\left(b_{21}^{2}+b_{22}^{2}\right)^{2},
\end{aligned}
$$


bem como

$$
\begin{aligned}
E\left[\left(C_{33}\right)^{4}\right]= & E\left\{\left(b_{31} Z_{\bar{u}_{t .}}^{2}+b_{32} Z_{\bar{u}_{t .}} Z_{e_{t}}+R_{33 t}\right)^{4}\right\} \\
= & 105 b_{31}^{4}+9 b_{32}^{4}+E\left(R_{33 t}^{4}\right)+4 b_{31} E\left(R_{33 t}^{3}\right)+60 b_{31}^{3} E\left(R_{33 t}\right) \\
& +90 b_{31}^{2} b_{32}^{2}+6\left(3 b_{31}^{2}+b_{32}^{2}\right) E\left(R_{33 t}^{2}\right)+36 b_{31} b_{32}^{2} E\left(R_{3 t t}\right) .
\end{aligned}
$$

II) Cálculo de $\sum_{i>j}^{3} \sum_{j=1}^{2} E\left(C_{3 j}^{3} c_{3 i}+c_{3 j} C_{3 i}^{3}\right)$

Façamos $S U 1=\sum_{i>j}^{3} \sum_{j=1}^{2} E\left(C_{3 j}^{3} C_{3 i}\right)$, logo, temos

$$
\begin{aligned}
S U 1= & E\left\{\left(b_{11} Z_{x_{t}}^{2}\right)^{3}\left[\left(b_{21} z_{\bar{u} t}+b_{22} Z_{e_{t}}\right) Z_{x_{t}}+\left(b_{31} Z_{\bar{u}_{t .}}^{2}+b_{32} Z_{\bar{u}_{t}} Z_{e_{t}}+R_{33 t}\right)\right]\right\} \\
& +E\left\{\left[\left(b_{21} Z_{\bar{u}_{t .}}+b_{22} Z_{e_{t}}\right) Z_{x_{t}}\right]^{3}\left[b_{31} Z_{\bar{u}_{t},}^{2}+b_{32} Z_{\bar{u}_{t .}} Z_{e_{t}}+R_{33 t}\right]\right\} \\
= & 15 b_{11}^{3}\left[b_{31}+E\left(R_{33 t}\right)\right]
\end{aligned}
$$

e

$$
\begin{aligned}
\sum_{i>j}^{3} \sum_{j=1}^{2} E\left(C_{3 j} C_{3 i}^{3}\right)= & E\left(C_{31} C_{33}^{3}\right) \\
= & E\left\{\left(b_{11} Z_{x_{t}}^{2}\right)\left[\left(b_{31} Z_{\bar{u}_{t .}}^{2}+b_{32} Z_{\bar{u}_{t .}} Z_{e_{t}}+R_{33 t}\right)^{3}\right]\right\} \\
= & b_{11}\left[15 b_{31}^{3}+E\left(R_{33 t}^{3}\right)+\left(9 b_{31}^{2}+3 b_{32}^{2}\right) E\left(R_{33 t}\right)\right. \\
& \left.+9 b_{31} b_{32}^{2}+3 b_{31} E\left(R_{33 t}^{2}\right)\right] .
\end{aligned}
$$

III) Cálculo de $E\left(\sum_{i>j}^{3} \sum_{j=1}^{2} C_{3 j}^{2} C_{3 i}^{2}\right)$

Temos que

$$
\begin{aligned}
E\left(C_{31}^{2} C_{32}^{2}\right) & =E\left\{\left[\left(b_{11} Z_{x_{t}}^{2}\right)^{2}\right]\left[\left(b_{21} Z_{\bar{u}_{t .}}+b_{22} Z_{e_{t}}\right)^{2}\left(Z_{x_{t}}\right)^{2}\right]\right\} \\
& =15 b_{11}^{2}\left(b_{21}^{2}+b_{22}^{2}\right) ; \\
E\left(C_{31}^{2} C_{33}^{2}\right) & =E\left(C_{31}^{2}\right) E\left(C_{33}^{2}\right) \\
& =3 b_{11}^{2}\left\{3 b_{31}^{2}+b_{32}^{2}+E\left(R_{33 t}^{2}\right)+2 b_{31} E\left(R_{33 t}\right)\right\} ;
\end{aligned}
$$


e

$$
\begin{aligned}
E\left(C_{32}^{2} C_{33}^{2}\right)= & E\left[\left(b_{21} z_{\bar{u} t .}+b_{22} Z_{e_{t}}\right)^{2}\left(Z_{x_{t}}\right)^{2}\left(b_{31} Z_{\bar{u}_{t .}}^{2}+b_{32} Z_{\bar{u} t .} Z_{e_{t}}+R_{33 t}\right)^{2}\right] \\
= & b_{21}^{2}\left[15 b_{31}^{2}+3 b_{32}^{2}+E\left(R_{33 t}^{2}\right)\right]+b_{22}^{2}\left[3 b_{31}^{2}+3 b_{32}^{2}+E\left(R_{33 t}^{2}\right)\right] \\
& +6 b_{11}^{2} b_{31} E\left(R_{33 t}\right)+3 b_{21}^{2} b_{31} E\left(R_{33 t}\right) \\
& +b_{22}^{2} b_{31} E\left(R_{33 t}\right)+4 b_{21} b_{22}\left[3 b_{31} b_{32}+b_{32} E\left(R_{33 t}\right)\right] .
\end{aligned}
$$

IV) Cálculo de $E\left(C_{31}^{2} C_{32} C_{33}+C_{31} C_{32}^{2} C_{33}+C_{31} C_{32} C_{33}^{2}\right)$

Temos que

$$
\begin{aligned}
E\left(C_{31}^{2} C_{32} C_{33}\right. & \left.+C_{31} C_{32}^{2} C_{33}+C_{31} C_{32} C_{33}^{2}\right)=E\left(C_{31} C_{32}^{2} C_{33}\right) \\
& =E\left[\left(b_{11} z_{x t}^{2}\right)\left(b_{21} Z_{\bar{u} t .}+b_{22} Z_{e t}\right)^{2}\left(Z_{x_{t}}\right)^{2}\left(b_{31} Z_{\bar{u}_{t .}}^{2}+b_{32} Z_{\bar{u}_{t .}} z_{e_{t}}+R_{33 t}\right)\right] \\
& =3 b_{11}\left\{\left(b_{21}^{2}+b_{22}^{2}\right)\left[b_{31}+E\left(R_{33 t}^{2}\right)\right]+2 b_{21}^{2} b_{31}+2 b_{21} b_{22} b_{32}\right\} \\
& =3 b_{11}\left[\left(3 b_{21}^{2}+b_{22}^{2}\right) b_{31}+\left(b_{21}^{2}+b_{22}\right) E\left(R_{33 t}\right)+2 b_{21} b_{22} b_{32}\right] . \quad \text { (C.96) }
\end{aligned}
$$

Agora, com os resultados obtidos acima, podemos concluir os cálculos anunciados anteriormente. Assim, substituindo (C.88), (C.89) e (C.90) em (C.87), obtemos

$$
\text { I) } \begin{aligned}
\sum_{i=1}^{3} E\left(C_{3 i}^{4}\right)= & 105 b_{11}^{4}+9 b_{21}^{4}+9 b_{22}^{4} 105 b_{31}^{4}+9 b_{32}^{4}+E\left(R_{33 t}^{4}\right)+4 b_{31} E\left(R_{33 t}^{3} .\right. \\
& +60 b_{31}^{3} E\left(R_{33 t}\right)+18 b_{21}^{2} b_{22}^{2}+90 b_{31}^{2} b_{32}^{2}+18 b_{31}^{2} E\left(R_{33}^{2}\right) \\
& +6 b_{32}^{2} E\left(R_{33 t}^{2}\right)+36 b_{31} b_{32}^{2} E\left(R_{33 t}\right) .
\end{aligned}
$$

De (C.91) e (C.92) vem

$$
\begin{aligned}
\text { II) } \sum_{i>j}^{3} \sum_{j=1}^{2} E\left(C_{3 j}^{3} C_{3 i}+C_{3 j} C_{3 i}^{3}\right)= & 15 b_{11}^{3} b_{31}+15 b_{11}^{3} E\left(R_{33 t}\right)+b_{11}\left[15 b_{31}^{3}+E\left(R_{33 t}^{3}\right)\right] \\
& +b_{11}\left(9 b_{31}^{2}+3 b_{32}^{2}\right) E\left(R_{33 t}\right)+9 b_{11} b_{31} b_{32}^{2} \\
& +3 b_{11} b_{31} E\left(R_{33 t}^{2}\right) .
\end{aligned}
$$

De (C.93), (C.94) e (C.95), obtemos 


$$
\text { III } \begin{aligned}
E\left(\sum_{i>j}^{3} \sum_{j=1}^{2} C_{3 j}^{2} C_{3 i}^{2}\right)= & b_{11}^{2}\left[15 b_{21}^{2}+15 b_{22}^{2}+9 b_{31}^{2}+3 b_{32}^{2}+3 E\left(R_{33 t}^{2}\right)\right] \\
& +b_{21}^{2}\left[15 b_{31}^{2}+3 b_{32}^{2}+E\left(R_{33 t}^{2}\right)\right]+b_{22}^{2}\left[3 b_{31}^{2}+3 b_{32}^{2}+E\left(R_{33 t}^{2}\right)\right] \\
& +12 b_{11}^{2} b_{31} E\left(R_{33 t}\right)+3 b_{21}^{2} b_{31} E\left(R_{33 t}\right) \\
& +b_{22}^{2} b_{31} E\left(R_{33 t}\right)+4 b_{21} b_{22}\left[3 b_{31} b_{32}+b_{32} E\left(R_{33 t}\right)\right] .
\end{aligned}
$$

Façamos $S U 2=E\left(C_{31}^{2} C_{32} C_{33}+C_{31} C_{32}^{2} C_{33}+C_{31} C_{32} C_{33}^{2}\right)$. Logo, de (C.96), obtemos $I V) S U 2=3 b_{11}\left[\left(3 b_{21}^{2}+b_{22}^{2}\right)\right] b_{31}+3 b_{11}\left(b_{21}^{2}+b_{22}^{2}\right) E\left(R_{33 t}\right)+6 b_{11} b_{21} b_{22} b_{32}$.

Substituindo (C.97) a (C.100) em (C.86), obtemos

$$
\begin{aligned}
E\left[\left(U_{t 3}\right)^{4}\right]= & \left\{\left[105 b_{11}^{4}+9 b_{21}^{4}+9 b_{22}^{4}+105 b_{31}^{4}+9 b_{32}^{4}+E\left(R_{33 t}^{4}\right)\right]\right. \\
& +4\left[b_{31} E\left(R_{33 t}^{3}\right)+15 b_{31}^{3} E\left(R_{33 t}\right)\right] \\
& \left.+6\left[3 b_{21}^{2} b_{22}^{2}+15 b_{31}^{2} b_{32}^{2}+3 b_{31}^{2} E\left(R_{33 t}^{2}\right)+b_{32}^{2} E\left(R_{33 t}^{2}\right)\right]+\left[36 b_{31} b_{32}^{2} E\left(R_{33 t}\right)\right]\right\} \\
& +4\left\{\left[15 b_{11}^{3}\left(b_{31}+E\left(R_{33 t}\right)\right)+b_{11}\left(15 b_{31}^{3}+E\left(R_{33 t}^{3}\right)\right)\right]\right. \\
& \left.+\left[b_{11}\left(9 b_{31}^{2}+3 b_{32}^{2}\right) E\left(R_{33 t}\right)+9 b_{11} b_{31} b_{32}^{2}+3 b_{11} b_{31} E\left(R_{33 t}^{2}\right)\right]\right\} \\
& +6\left\{\left[b_{11}^{2}\left[15 b_{21}^{2}+15 b_{22}^{2}+9 b_{31}^{2}+3 b_{32}^{2}+3 E\left(R_{33 t}^{2}\right)\right]\right.\right. \\
& \left.+b_{21}^{2}\left[15 b_{31}^{2}+3 b_{32}^{2}+E\left(R_{33 t}^{2}\right)\right]+b_{22}^{2}\left[3 b_{31}^{2}+3 b_{32}^{2}+E\left(R_{33 t}^{2}\right)\right]\right] \\
& +\left[12 b_{11}^{2} b_{31} E\left(R_{33 t}\right)+3 b_{21}^{2} b_{31} E\left(R_{33 t}\right)+b_{22}^{2} b_{31} E\left(R_{33 t}\right)\right] \\
& \left.+\left[4 b_{21} b_{22}\left[3 b_{31} b_{32}+b_{32} E\left(R_{33 t}\right)\right]\right]\right\} \\
& +12\left\{\left[3 b_{11}\left(3 b_{21}^{2}+b_{22}^{2}\right) b_{31}+3 b_{11}\left(b_{21}^{2}+b_{22}^{2}\right) E\left(R_{33 t}\right)\right]+\left[6 b_{11} b_{21} b_{22} b_{32}\right]\right\}
\end{aligned}
$$


De (C.101), temos

$$
\begin{aligned}
E\left[\left(U_{t 3}\right)^{4}\right] \leq & 105\left\{\left[b_{11}^{4}+b_{21}^{4}+b_{22}^{4}+b_{31}^{4}+b_{32}^{4}+E\left(R_{33 t}^{4}\right)\right]\right. \\
& +\left[\left|b_{11}^{3}\right|\left(\left|b_{31}\right|+E\left(R_{33 t}\right)\right)+\left|b_{31}^{3}\right| E\left(R_{33 t}\right)+\left|b_{11}\right|\left(\left|b_{31}\right|^{3}+E\left(R_{33 t}^{3}\right)\right)\right. \\
& \left.+\left|b_{31}\right| E\left(R_{33 t}^{3}\right)\right]+\left[b_{11}^{2}\left(b_{21}^{2}+b_{22}^{2}+b_{31}^{2}+b_{32}^{2}+E\left(R_{33 t}^{2}\right)\right)\right. \\
& +b_{21}^{2}\left(b_{22}^{2}+b_{31}^{2}+b_{32}^{2}+E\left(R_{33 t}^{2}\right)\right)+b_{22}^{2}\left(b_{31}^{2}+b_{32}^{2}+E\left(R_{33 t}^{2}\right)\right) \\
& \left.+b_{31}^{2}\left(b_{32}^{2}+E\left(R_{33 t}^{2}\right)+b_{32}^{2} E\left(R_{33 t}\right)\right)\right]+\left[b_{11}^{2}\left|b_{31}\right| E\left(R_{33 t}\right)\right. \\
& +b_{21}^{2}\left|b_{31}\right| E\left(R_{33 t}\right)+b_{22}^{2}\left|b_{31}\right| E\left(R_{33 t}\right)+\left|b_{11}\right|\left(b_{21}^{2}+b_{22}^{2}\right)\left|b_{31}\right| \\
& +\left|b_{11}\right|\left(b_{21}^{2}+b_{22}^{2}\right) E\left(R_{33 t}\right)+\left|b_{11}\right|\left(b_{31}^{2}+b_{32}^{2}\right) E\left(R_{33 t}\right) \\
& \left.+\left|b_{31}\right| b_{32}^{2} E\left(R_{33 t}\right)+\left|b_{11} b_{31}\right|\left(b_{32}^{2}+E\left(R_{33 t}^{2}\right)\right)\right] \\
& \left.+\left|b_{21} b_{22}\right|\left[\left|b_{31} b_{32}\right|+\left|b_{32}\right| E\left(R_{33 t}\right)\right]+\left|b_{11} b_{21} b_{22} b_{32}\right|\right\}
\end{aligned}
$$

Usando os resultados obtidos na seção anterior (C.81):

$$
\begin{aligned}
b_{33 t} & =\left[\left|b_{33}\right| 2\left(r_{t}+1\right) E\left(R_{3 t}\right)+\left|E\left(R_{33 t}\right)\right|\right] \\
E\left(R_{33 t}\right) & \leq\left|a_{1} \sigma_{\bar{X} \bar{X} t}+a_{2} \sigma_{\bar{X} Y}\right| \leq b_{33 t} \\
E\left(R_{33 t}^{2}\right) & <\left[\left|a_{33}\right|\left[2\left(r_{t}+1\right) E\left(R_{3 t}\right)\right]+\left|E\left(R_{33 t}\right)\right|\right]^{2}=b_{33 t}^{2} \\
E\left(R_{33 t}^{3}\right) & <\left[\left|a_{33}\right|\left[2\left(r_{t}+1\right) E\left(R_{3 t}\right)\right]+\left|E\left(R_{33 t}\right)\right|\right]^{3}=b_{33 t}^{3} \\
E\left(R_{33 t}^{4}\right) & <\left[\left|a_{33}\right|\left[2\left(r_{t}+1\right) E\left(R_{3 t}\right)\right]+\left|E\left(R_{33 t}\right)\right|\right]^{4}=b_{33 t}^{4}
\end{aligned}
$$

e substituindo em (C.102), e com a suposição adotada ao longo deste trabalho de que $r_{t} \leq r<\infty$, obtemos

$$
E\left(U_{3 t}^{4}\right)<105\left[\left|b_{11}\right|+\left|b_{22}\right|+\left|b_{31}\right|+\left|b_{32}\right|+\left|b_{33 t}\right|\right]^{4}<\infty .
$$

Analogamente prova-se que:

$$
\begin{aligned}
& E\left(U_{2 t}^{4}\right)<\infty \\
& e \\
& E\left(U_{1 t}^{4}\right)<\infty .
\end{aligned}
$$




\section{C.4 Prova que $\mathbf{E}\left\{\left|\mathbf{U}_{\mathrm{it}}\right|^{3}\right\}<\infty, i=1,2,3$.}

Os cálculos desta seção são utilizados para mostrar que as seqüências das variáveis acima satisfazem as condições do teorema central do limite de Liapounov. Novamente, aqui, só apresentaremos os cálculos referentes à variável $U_{3 t}$, pelos motivos já apresentados. Note que

$$
U_{3 t}=b_{11} Z_{x_{t}}^{2}+\left(b_{21} Z_{\bar{u}_{t .}}+b_{22} Z_{e_{t}}\right) Z_{x_{t}}+b_{31} Z_{\bar{u}_{t .}}^{2}+b_{32} Z_{\bar{u}_{t .}} Z_{e_{t}}+a_{33}^{*} R_{3 t}-A .
$$

Os coeficientes que aparecem na expressão acima já foram definidos em (C.85). Temos que:

$$
\begin{aligned}
\left|U_{3 t}\right| \leq U_{3 t}^{*}= & b_{11}^{*} Z_{x_{t}}^{2}+\left(b_{21}^{*}\left|Z_{\bar{u}_{t .}}\right|+b_{22}^{*}\left|Z_{e_{t}}\right|\right)\left|Z_{x_{t}}\right|+b_{31}^{*} Z_{\bar{u}_{t .}}^{2}+b_{32}\left|Z_{\bar{u}_{t .}} Z_{e_{t}}\right| \\
& +a_{33}^{*} R_{3 t}+|A| .
\end{aligned}
$$

com:

$$
\begin{aligned}
& b_{11}^{*}=\left|\left(a_{1}+a_{2} \beta\right)\right| \sigma_{x x} ; \quad b_{21}^{*}=\left|\left(2 a_{1}+a_{2} \beta\right)\right| \sqrt{\frac{\sigma_{x x} \sigma_{u u}}{r_{t}}} ; \quad b_{22}^{*}=\left|a_{2}\right| \sqrt{\sigma_{x x} \sigma_{e e}} \\
& b_{31}^{*}=\left|a_{1}\right| \frac{\sigma_{u u}}{r_{t}} ; b_{32}^{*}=\left|a_{2}\right| \sqrt{\frac{\sigma_{e e} \sigma_{u u}}{r_{t}}} ; \quad b_{33}^{*}=\left|a_{3}\right| \nu_{n} R_{3 t}=\left|a_{3}\right| \frac{\sum_{t=1}^{n} \frac{1}{r_{t}}}{\sum_{t=1}^{n}\left(r_{t}-1\right)} R_{3 t} .
\end{aligned}
$$

Façamos

$$
\begin{aligned}
& C_{31}^{*}=b_{11}^{*} Z_{x_{t}}^{2}, \quad C_{32}^{*}=\left(b_{21}^{*}\left|Z_{\bar{u}_{t .}}\right|+b_{22}^{*}\left|Z_{e_{t}}\right|\right)\left|Z_{x_{t}}\right| \quad \mathrm{e} \\
& C_{33}^{*}=b_{31}^{*} Z_{\bar{u}_{t .}}^{2}+b_{32}^{*}\left|Z_{\bar{u}_{t} .} Z_{e_{t}}\right|+b_{33}^{*}+|A| .
\end{aligned}
$$

Logo, temos que:

$$
U_{3 t}^{*}=C_{31}^{*}+C_{32}^{*}+C_{33}^{*}
$$

Consideremos, agora, a seguinte proposição (James, 1999, Prop-3.2):

"Sendo $0<s<t$, a função $\varphi(y)=|y|^{t / s}$ é convexa, pois, $t / s>1$. Se Y é integrável a desiqualdade de Jensen implica que $E\left\{|Y|^{t / s}\right\} \geq|E Y|^{t / s}$. Façamos $Y=|X|^{s}$, 
se $|X|^{s}$ é integrável, então $\left(E|X|^{s}\right)^{t / s} \leq E\left\{|X|^{t}\right\}$, ou equivalentemente, $E\left\{|X|^{s}\right\} \leq$ $\left(\left.|E| X\right|^{t}\right)^{s / t} "$.

Para ilustrar a proposição consideremos o seguinte exemplo: $E\left\{|Z|^{3}\right\} \leq\left(E\left(Z^{4}\right)^{3 / 4}=\right.$ $(3)^{3 / 4}$.

Usando a proposição acima e o mesmo procedimento utilizado para calcular $E\left(U_{3 t}^{4}\right)$, podemos mostrar que: :

$$
\begin{aligned}
E\left\{\left|U_{3 t}\right|^{3}\right\} \leq & E\left(U_{3 t}^{*}\right)=E\left(C_{31}^{*}+C_{32}^{*}+C_{33}^{*}\right)^{3} \leq 15\left(b_{11}^{*}+b_{11}^{*}+b_{21}^{*}+b_{31}^{*}+b_{32}^{*}\right. \\
& \left.+\left|a_{3}\right|\left(r_{t}-1\right) \sigma_{u u}+|A|\right)^{3} \\
\leq & 15\left(a^{*}\right)^{3}\left[2 \sigma_{\bar{X}_{\bar{X}}}+2 \sigma_{\bar{X} \bar{X}_{t}}+2\left(r_{t}-1\right) \sigma_{u u}+(2+\beta) \sqrt{\frac{\sigma_{x x} \sigma_{u u}}{r_{t}}}\right. \\
& \left.+\sqrt{\sigma_{e e}}\left(\sqrt{\sigma_{x x}}+\sqrt{\frac{\sigma_{u u}}{r_{t}}}\right)\right]^{3}<\infty
\end{aligned}
$$

em que $a^{*}=\max \left(\left|a_{1}\right|,\left|a_{1}\right|,\left|a_{1}\right|\right)$.

Analogamente, provamos que:

$$
\begin{aligned}
E\left\{\left|U_{2 t}\right|^{3}\right\} \leq & 15\left\{2\left|a_{1}\right| \sigma_{\bar{X} \bar{X}_{t}}+2\left|a_{1}\right| \sigma_{\bar{X} Y}+\left(2\left|a_{1}\right|+\left|a_{2}\right||\beta|\right) \sqrt{\frac{\sigma_{x x} \sigma_{u u}}{r_{t}}}\right. \\
& \left.+\left|a_{2}\right| \sqrt{\sigma_{e e}}\left(\sqrt{\sigma_{x x}}+\sqrt{\frac{\sigma_{u u}}{r_{t}}}\right)+\left|a_{3}\right| \nu_{4 n} I_{r_{t}}(1) \sigma_{u u}\left(\sqrt{\frac{r_{t}+1}{r_{t}-1}}+1\right)^{2}\right\}^{3}<\infty
\end{aligned}
$$

onde $\nu_{4 n}=\frac{\sum_{t=1}^{n} \frac{1}{r_{t}}}{\sum_{t=1}^{n} I_{r_{t}}(1)}$

e, também, que

$$
\begin{aligned}
E\left\{\left|U_{1 t}-E\left(U_{1 t}\right)\right|^{3}\right\}= & E\left\{\left|U_{1 t}\right|^{3}\right\} \leq 15 a_{m}^{3}\left[\sigma_{X X}+2 \sigma_{X Y}+(2+|\beta|) \sqrt{\sigma_{u u} \sigma_{x x}}\right. \\
& \left.+\sqrt{\sigma_{e e}}\left(\sqrt{\sigma_{x x}}+\sigma_{u u}\right)\right]^{3}<\infty,
\end{aligned}
$$

onde $a_{m}=\max \left(\left|a_{1}\right|,\left|a_{2}\right|,\left|a_{3}\right| \nu_{1 n} I_{r_{t}}(1)\right)$ e $\quad \nu_{1 n}=\frac{n}{\sum_{t=1}^{n} I_{r_{t}}(1)}$. 


\section{C.5 Cálculo de $\operatorname{Var}\left(U_{i t}\right), i=1,2,3$.}

Usando as notações da Seção C.3, temos que $E\left\{U_{3 t}\right\}=0$, por construção, e

$$
\begin{aligned}
\operatorname{Var}\left\{U_{3 t}\right\} & =E\left\{\left(U_{3 t}\right)^{2}\right\}=E\left\{\left(C_{31}+C_{32}+C_{33}\right)^{2}\right\} \\
& =E\left\{\sum_{i=1}^{3}\left(C_{3 i}\right)^{2}+2 \sum_{j=1}^{2} \sum_{i>j}^{3} C_{3 i} C_{3 j}\right\} .
\end{aligned}
$$

\section{Cálculos preliminares}

(I) Cálculo de $E\left\{\sum_{i=1}^{3}\left(C_{3 i}^{2}\right)\right\}$

$$
\begin{aligned}
E\left\{(C 31)^{2}\right\} & =E\left\{\left(b_{11} Z_{x_{t}}^{2}\right)^{2}\right\}=3 b_{11}^{2} \\
E\left\{(C 32)^{2}\right\} & =E\left\{\left[\left(b_{21} Z_{\bar{u}_{t .}}+b_{22} Z_{e_{t}}\right) Z_{x_{t}}\right]^{2}\right\}=E\left(Z_{x_{t}}^{2}\right) E\left\{\left(b_{21} Z_{\bar{u}_{t .}}+b_{22} Z_{e_{t}}\right)^{2}\right\} \\
& =b_{21}^{2} E\left(Z_{\bar{u}_{t}}^{2}\right)+b_{22}^{2} E\left(Z_{e_{t}}^{2}\right)+2 b_{21} b_{22} E\left(Z_{\bar{u}_{t .}} Z_{e_{t}}\right)=b_{21}^{2}+b_{22}^{2}
\end{aligned}
$$

$$
\begin{aligned}
E\left\{(C 33)^{2}\right\}= & E\left(b_{31} Z_{\bar{u}_{t .}}^{2}+b_{32} Z_{\bar{u}_{t .}} Z_{e_{t}}+R_{33 t}\right)^{2} \\
= & b_{31}^{2} E\left(Z_{\bar{u}_{t .}}^{4}\right)+b_{32}^{2} E\left(Z_{\bar{u}_{t .}}^{2}\right) E\left(Z_{e_{t}}^{2}\right)+E\left(R_{33 t}\right)^{2}+ \\
& +2 b_{31} b_{32} E\left[\left(Z_{\bar{u}_{t .}}^{2}\right)\left(Z_{\bar{u}_{t .}} Z_{e_{t}}\right)\right]+2 b_{31} E\left[\left(Z_{\bar{u}_{t .}}^{2}\right)\left(R_{33 t}\right)\right] \\
& +2 b_{32} E\left[\left(Z_{\bar{u}_{t}} Z_{e_{t}}\right)\left(R_{33 t}\right)\right]
\end{aligned}
$$

$$
=3 b_{31}^{2}+b_{32}^{2}+2 a_{33}^{2}\left(r_{t}-1\right)^{-1}\left[E\left(R_{33 t}\right)\right]^{2}+\left[E\left(R_{33 t}\right)\right]^{2}+2 b_{31}\left[E\left(R_{33 t}\right)\right] .
$$

De (C.115) a (C.117), temos:

$$
\begin{aligned}
E\left\{\sum_{i=1}^{3}\left(C_{3 i}^{2}\right)\right\}= & 3 b_{11}^{2}+b_{21}^{2}+b_{22}^{2}+3 b_{31}^{2}+b_{32}^{2}+\left(a_{33}\right)^{2} \operatorname{Var}(R 3 t)+\left[E\left(R_{33 t}\right)\right]^{2} \\
& +2 b_{31}\left[E\left(R_{33 t}\right)\right] .
\end{aligned}
$$


(II) Cálculo de $E\left\{\sum_{j=1}^{2} \sum_{i>j}^{3}\left(C_{3 i} C_{3 j}\right)\right\}=S I I$.

$$
\begin{aligned}
S I I= & E\left\{C_{31}\left(C_{32}+C_{33}\right)+C_{32} C_{33}\right\} \\
= & b_{11} E\left\{\left(Z_{x_{t}}^{2}\right)\left(b_{21} Z_{\bar{u}_{t .}}+b_{22} Z_{e_{t}}\right) Z_{x_{t}}\right\}+b_{11} E\left\{( Z _ { x _ { t } } ^ { 2 } ) \left(b_{31} Z_{\bar{u}_{t .}}^{2}+b_{32} Z_{\bar{u}_{t .}} Z_{e_{t}}\right.\right. \\
& \left.\left.+R_{33 t}\right)\right\}+E\left\{\left[\left(b_{21} Z_{\bar{u}_{t .}}+b_{22} Z_{e_{t}}\right) Z_{x_{t}}\right]\left(b_{31} Z_{\bar{u}_{t .}}^{2}+b_{32} Z_{\bar{u}_{t .}} Z_{e_{t}}+R_{33 t}\right)\right\} \\
= & b_{11}\left[b_{31}+E\left(R_{33 t}\right)\right] .
\end{aligned}
$$

Substituindo (C.118) e (C.119) em (C.114), temos

$$
\begin{aligned}
\operatorname{Var}\left\{U_{3 t}\right\}= & E\left\{U_{3 t}^{2}\right\} \\
= & 3 b_{11}^{2}+b_{21}^{2}+b_{22}^{2}+3 b_{31}^{2}+b_{32}^{2}+\left(a_{33}^{*}\right)^{2} \operatorname{Var}\left(R_{3 t}\right)+\left[E\left(R_{33 t}\right)\right]^{2} \\
& +2 b_{31}\left[E\left(R_{33 t}\right)\right]++2 b_{11} b_{31}+2 b_{11} E\left(R_{33 t}\right) .
\end{aligned}
$$

Mas,

$$
\begin{aligned}
E\left(R_{33 t}\right) & =-\left(a_{1} \sigma_{\bar{X} \bar{X}_{t}}+a_{2} \sigma_{\bar{X} \bar{Y}}\right) \\
b_{11}^{2} & =a_{1}^{2} \sigma_{x x}^{2}+a_{2}^{2} \beta^{2} \sigma_{x x}^{2}+2 a_{1} a_{2} \beta^{2} \sigma_{x x}^{2} \\
b_{21}^{2} & =\left(4 a_{1}^{2}+a_{2}^{2} \beta^{2}+4 a_{1} a_{2} \beta\right)\left(\frac{\sigma_{u u}}{r_{t}}\right), \\
b_{22}^{2} & =\left(a_{2}^{2} \sigma_{x x} \sigma_{e e}\right) \\
b_{31}^{2} & =a_{1}^{2}\left(\frac{\sigma_{u u}^{2}}{r_{t}^{2}}\right) \\
b_{32}^{2} & =a_{2}^{2}\left(\frac{\sigma_{u u} \sigma_{e e}}{r_{t}}\right) \\
\left(a_{33}\right)^{2} & =a_{3}^{2} \nu_{n}^{2}, \\
b_{11} b_{31} & =a_{1}\left(a_{1}+a_{2} \beta\right)\left(\frac{\sigma_{x x} \sigma_{u u}}{r_{t}}\right) .
\end{aligned}
$$


Substituindo (C.121) - (C.127) em (C.120), temos

$$
\begin{aligned}
& \operatorname{Var}\left\{U_{3 t}\right\}=E\left\{U_{3 t}^{2}\right\}=3 a_{1}^{2} \sigma_{x x}^{2}+3 a_{2}^{2} \beta^{2} \sigma_{x x}^{2}+6 a_{1} a_{2} \beta \sigma_{x x}^{2}+\left(4 a_{1}^{2}+a_{2}^{2} \beta^{2}+4 a_{1} a_{2} \beta\right) \times \\
& \times\left(\frac{\sigma_{x x} \sigma_{u u}}{r_{t}}\right)+\left(a_{2}^{2} \sigma_{x x} \sigma_{e e}\right)+3 a_{1}^{2}\left(\frac{\sigma_{u u}^{2}}{r_{t}^{2}}\right)+a_{2}^{2}\left(\frac{\sigma_{u u} \sigma_{e e}}{r_{t}}\right) \\
& +\left(a_{3}^{2} \hat{\lambda}^{2}\right) \operatorname{Var}\left(R_{3 t}\right)+a_{1}^{2} \sigma_{\bar{X} \bar{X}_{t}}^{2}+a_{2}^{2} \sigma_{\bar{X} \bar{Y}}^{2}+2 a_{1} a_{2} \sigma_{\bar{X} \bar{X}_{t}} \sigma_{\bar{X} \bar{Y}} \\
& +2 a_{1}\left(\frac{\sigma_{u u}}{r_{t}}\right) E\left(R_{33 t}\right)+2 a_{1}\left(a_{1}+a_{2} \beta\right)\left(\frac{\sigma_{x x} \sigma_{u u}}{r_{t}}\right) \\
& +2\left(a_{1}+a_{2} \beta\right) \sigma_{x x} E\left(R_{33 t}\right) \text {. }
\end{aligned}
$$

Podemos reescrever a expressão acima como segue

$$
\begin{aligned}
\operatorname{Var}\left\{U_{3 t}\right\}= & E\left\{U_{3 t}^{2}\right\}=3 a_{1}^{2} \sigma_{x x}^{2}+3 a_{2}^{2} \beta^{2} \sigma_{x x}^{2}+6 a_{1} a_{2} \beta \sigma_{x x}^{2}+\left(4 a_{1}^{2}\right)\left(\frac{\sigma_{x x} \sigma_{u u}}{r_{t}}\right) \\
& +\left(a_{2}^{2} \beta^{2}\right)\left(\frac{\sigma_{x x} \sigma_{u u}}{r_{t}}\right)+4 a_{1} a_{2} \beta\left(\frac{\sigma_{x x} \sigma_{u u}}{r_{t}}\right)+\left(a_{2}^{2} \sigma_{x x} \sigma_{e e}\right)+3 a_{1}^{2}\left(\frac{\sigma_{u u}^{2}}{r_{t}^{2}}\right) \\
& +a_{2}^{2}\left(\frac{\sigma_{u u} \sigma_{e e}}{r_{t}}\right)+\left(a_{3}^{2} \nu_{n}^{2}\right) \operatorname{Var}\left(R_{3 t}\right)+\left[E\left(R_{33 t}\right)\right]^{2}+2 a_{1}\left(\frac{\sigma_{u u}}{r_{t}}\right) E\left(R_{33 t}\right) \\
& +2 a_{1}^{2}\left(\frac{\sigma_{x x} \sigma_{u u}}{r_{t}}\right)+2 a_{1} a_{2} \beta\left(\frac{\sigma_{x x} \sigma_{u u}}{r_{t}}\right)+2\left(a_{1}+a_{2} \beta\right) \sigma_{x x} E\left(R_{33 t}\right) .
\end{aligned}
$$

Sejam

$$
\begin{aligned}
& S U 1=3 a_{1}^{2} \sigma_{x x}^{2}+3 a_{1}^{2}\left(\frac{\sigma_{u u}^{2}}{r_{t}^{2}}\right)+2 a_{1}^{2}\left(\frac{\sigma_{x x} \sigma_{u u}}{r_{t}}\right)+4 a_{1}^{2}\left(\frac{\sigma_{x x} \sigma_{u u}}{r_{t}}\right) ; \\
& S U 2=3 a_{2}^{2} \beta^{2} \sigma_{x x}^{2}+\left(a_{2}^{2} \beta^{2}\right)\left(\frac{\sigma_{x x} \sigma_{u u}}{r_{t}}\right)+\left(a_{2}^{2} \sigma_{x x} \sigma_{e e}\right)+a_{2}^{2}\left(\frac{\sigma_{u u} \sigma_{e e}}{r_{t}}\right) ; \\
& S U 3=6 a_{1} a_{2} \beta \sigma_{x x}^{2}+4 a_{1} a_{2} \beta\left(\frac{\sigma_{x x} \sigma_{u u}}{r_{t}}\right)+2 a_{1} a_{2} \beta\left(\frac{\sigma_{x x} \sigma_{u u}}{r_{t}}\right) \\
& \mathrm{e} \\
& S U 4=2 a_{1}\left(\frac{\sigma_{u u}}{r_{t}}\right) E\left(R_{33 t}\right)+2\left(a_{1}+a_{2} \beta\right) \sigma_{x x} E\left(R_{33 t}\right)+E\left(R_{33 t}\right)^{2} .
\end{aligned}
$$

Simplificando as expressões acima, temos

$$
S U 1=3 a_{1}^{2}\left(\sigma_{x x}^{2}+\frac{\sigma_{u u}^{2}}{r_{t}^{2}}+2 \frac{\sigma_{x x} \sigma_{u u}}{r_{t}}\right)=3 a_{1}^{2}\left(\sigma_{\bar{X} \bar{X}_{t}}\right)^{2},
$$




$$
\begin{aligned}
S U 2 & =2 a_{2}^{2} \beta^{2} \sigma_{x x}^{2}+a_{2}^{2} \beta^{2} \sigma_{x x}\left(\sigma_{x x}+\frac{\sigma_{u u}}{r_{t}}\right)+a_{2}^{2} \sigma_{e e}\left(\sigma_{x x}+\frac{\sigma_{u u}}{r_{t}}\right) \\
& =2 a_{2}^{2} \sigma_{\bar{X} Y}^{2}+a_{2}^{2} \beta^{2} \sigma_{x x} \sigma_{\bar{X} \bar{X}_{t}}+a_{2}^{2} \sigma_{e e} \sigma_{\bar{X} \bar{X}_{t}} \\
& =2 a_{2}^{2} \sigma_{\bar{X} Y}^{2}+a_{2}^{2} \sigma_{\bar{X} \bar{X}_{t}} \sigma_{Y Y}, \\
S U 3 & =6 a_{1} a_{2} \beta \sigma_{x x}\left(\sigma_{x x}+\frac{\sigma_{u u}}{r_{t}}\right)=6 a_{1} a_{2} \sigma_{\bar{X} \bar{X}_{t}} \sigma_{\bar{X} \bar{X}_{t}}, \\
S U 4 & =E\left(R_{33 t}\right)\left[2 a_{1}\left(\frac{\sigma_{u u}}{r_{t}}\right)+2\left(a_{1}+a_{2} \beta\right) \sigma_{x x}+E\left(R_{33 t}\right)\right] \\
& =E\left(R_{33 t}\right)\left[2 a_{1}\left(\frac{\sigma_{u u}}{r_{t}}+\sigma_{x x}\right)+2 a_{2} \beta \sigma_{x x}+E\left(R_{33 t}\right)\right] \\
& =E\left(R_{33 t}\right)\left[-2 E\left(R_{33 t}\right)+E\left(R_{33 t}\right)\right]=-\left[E\left(R_{33 t}\right)\right]^{2} \\
& =-\left[a_{1}^{2} \sigma_{\bar{X} \bar{X}_{t}}^{2}+a_{2}^{2} \sigma_{\bar{X} Y}^{2}+2 a_{1} a_{2} \sigma_{\bar{X} \bar{X}_{t}} \sigma_{\bar{X} Y}\right] .
\end{aligned}
$$

Como $\operatorname{Var}\left\{U_{3 t}\right\}=\left(a_{3}^{2} \nu_{n}^{2}\right) \operatorname{Var}\left(R_{3 t}\right)+S U 1+S U 2+S U 3+S U 4$, substituindo (C.130) - (C.133) em (C.129), temos

$$
\begin{aligned}
\operatorname{Var}\left\{U_{3 t}\right\}= & 3 a_{1}^{2} \sigma_{\bar{X} \bar{X}_{t}}^{2}+2 a_{2}^{2} \sigma_{\bar{X} Y}^{2}+a_{2}^{2} \sigma_{\bar{X} \bar{X}_{t}} \sigma_{Y Y}+6 a_{1} a_{2} \sigma_{\bar{X} \bar{X}_{t}} \sigma_{\bar{X} \bar{X}_{t},}-a_{1}^{2} \sigma_{\bar{X} \bar{X}_{t}}^{2}- \\
& -a_{2}^{2} \sigma_{\bar{X} Y}^{2}-2 a_{1} a_{2} \sigma_{\bar{X} \bar{X}_{t}} \sigma_{\bar{X} Y}+2 a_{3}^{2}\left(r_{t}-1\right) \nu_{n}^{2} \sigma_{u u}^{2} \\
= & 2 a_{1}^{2} \sigma_{\bar{X} \bar{X}_{t}}+a_{2}^{2} \sigma_{\bar{X} \bar{Y}}^{2}+4 a_{1} a_{2} \sigma_{\bar{X} \bar{Y}} \sigma_{\bar{X} \bar{X}_{t}}+a_{2}^{2} \sigma_{Y Y} \sigma_{\bar{X}_{\bar{X}}}+2 a_{3}^{2}\left(r_{t}-1\right) \hat{\lambda}^{2} \sigma_{u u}^{2} .
\end{aligned}
$$

Vamos obter $\operatorname{Var}\left\{U_{3 t}\right\}$ de uma outra maneira. Para tanto, façamos

$$
\begin{aligned}
W_{31} & =\left(\bar{X}_{t .}-\mu_{x}\right)^{2}-\sigma_{\bar{X} \bar{X}_{t}}, \\
W_{32} & =\left(\bar{X}_{t .}-\mu_{x}\right)\left(Y_{t}-\mu_{y}\right)-\sigma_{\bar{X} Y} \\
W_{33} & =\hat{\lambda}\left[R_{3 t}-\left(r_{t}-1\right) \sigma_{u u}\right]
\end{aligned}
$$

De (C.135), podemos escrever

$$
U_{3 t}=a_{1} W_{31}+a_{2} W_{32}+a_{3} W_{3} .
$$


Temos que

$$
\begin{aligned}
\operatorname{Var}\left(W_{31}\right) & =\operatorname{Var}\left\{\left(\bar{X}_{t .}-\mu_{x}\right)^{2}-\sigma_{\bar{X} \bar{X}_{t}}\right\}=\operatorname{Var}\left\{\left(\bar{X}_{t .}-\mu_{x}\right)^{2}\right\} \\
& =E\left\{\left(\bar{X}_{t .}-\mu_{x}\right)^{4}\right\}-\left\{E\left(\bar{X}_{t .}-\mu_{x}\right)^{2}\right\}^{2} \\
& =E\left\{\left(\frac{\left.\bar{X}_{t .}-\mu_{x}\right)}{\sqrt{\sigma_{\bar{X} \bar{X}_{t}}}}\right)^{4}\right\}\left(\sigma_{\bar{X} \bar{X}_{t}}\right)^{2}-\left[E\left(\frac{\left.\bar{X}_{t .}-\mu_{x}\right)}{\sqrt{\sigma_{\bar{X} \bar{X}_{t}}}}\right)^{2}\right]^{2}\left(\sigma_{\bar{X} \bar{X}_{t}}\right)^{2} \\
& =E\left\{\left(Z_{\bar{X}_{t .}}\right)^{4}\right\}\left(\sigma_{\bar{X} \bar{X}_{t}}\right)^{2}-\left[E\left\{\left(Z_{\bar{X}_{t .}}\right)^{2}\right\}\right]^{2}\left(\sigma_{\bar{X} \bar{X}_{t}}\right)^{2} \\
& =3 \sigma_{\bar{X} \bar{X}_{t}}-\sigma_{\bar{X} \bar{X}_{t}}=2 \sigma_{\bar{X} \bar{X}_{t}}
\end{aligned}
$$

$$
\begin{aligned}
& \operatorname{Cov}\{W 31, W 32\}=E\left[W_{31}-E\left(W_{31}\right)\right]\left[W_{32}-E\left(W_{32}\right)\right]=E\left(W_{31} W_{32}\right) \\
& \left.=E\left[\left(\bar{X}_{t .}-\mu_{x}\right)^{2}-\sigma_{\bar{X} \bar{X}_{t}}\right]\left[\left(\bar{X}_{t .}-\mu_{x}\right)\left(Y_{t}-\mu_{y}\right)-\sigma_{\bar{X} Y}\right)\right] \\
& \left.=E\left[\left(\bar{X}_{t .}-\mu_{x}\right)^{2}\right]\left[\left(\bar{X}_{t .}-\mu_{x}\right)\left(Y_{t}-\mu_{y}\right)-\sigma_{\bar{X} Y}\right)\right] \\
& \left.-\left(\sigma_{\bar{X} \bar{X}_{t}}\right) E\left[\left(\bar{X}_{t .}-\mu_{x}\right)\left(Y_{t}-\mu_{y}\right)-\sigma_{\bar{X} Y}\right)\right] \\
& \left.=E\left[\left(\bar{X}_{t .}-\mu_{x}\right)^{2}\right]\left[\left(\bar{X}_{t .}-\mu_{x}\right)\left(Y_{t}-\mu_{y}\right)\right]-\left(\sigma_{\bar{X} Y}\right)\right) E\left[\left(\bar{X}_{t .}-\mu_{x}\right)^{2}\right] \\
& =E\left[\left(\bar{X}_{t .}-\mu_{x}\right)^{3}\left(Y_{t}-\mu_{y}\right)\right]-\sigma_{\bar{X} Y} \sigma_{\bar{X} \bar{X}_{t}} \text {. }
\end{aligned}
$$

Fazendo $\mathrm{E}\left[\left(\bar{X}_{t .}-\mu_{x}\right)^{3}\left(Y_{t}-\mu_{y}\right)\right]=S W 12$, temos que

$$
\begin{aligned}
S W 12= & \mathrm{E}\left[\left(x_{t}-\mu_{x}\right)+\bar{u}_{t .}\right]^{3}\left[\alpha+\beta x_{t}+e_{t}-\left(\alpha+\beta \mu_{x}\right)\right] \\
= & \mathrm{E}\left\{\left[\left(x_{t}-\mu_{x}\right)+\bar{u}_{t .}\right]^{3}\left[\beta\left(x_{t}-\mu_{x}\right)\right]\right\}+\left\{E\left[\left(x_{t}-\mu_{x}\right)+\bar{u}_{t .}\right]^{3}\right\} E\left(e_{t}\right) \\
= & \mathrm{E}\left\{\left[\left(x_{t}-\mu_{x}\right)^{3}+\left(\bar{u}_{t .}\right)^{3}+3\left(x_{t}-\mu_{x}\right)^{2}\left(\bar{u}_{t .}\right)\right.\right. \\
& \left.\left.+3\left(x_{t}-\mu_{x}\right)\left(\bar{u}_{t .}\right)^{2}\right]\left[\beta\left(x_{t}-\mu_{x}\right)\right]\right\} \\
= & \left.3 \beta \sigma_{x x}^{2}+3 \beta E\left[\left(x_{t}-\mu_{x}\right)^{2}\left(\bar{u}_{t .}\right)^{2}\right)\right] \\
= & 3 \beta \sigma_{x x}^{2}+3 \beta \sigma_{x x}\left(\frac{\sigma_{u u}}{r_{t}}\right)=3 \beta \sigma_{x x}\left(\sigma_{x x}+\frac{\sigma_{u u}}{r_{t}}\right)=3 \sigma_{\bar{X} Y} \sigma_{\bar{X} \bar{X}_{t}} .
\end{aligned}
$$

Substituindo (C.139) em (C.138), temos

$$
\operatorname{Cov}\{W 31, W 32\}=2 \sigma_{\bar{X} Y} \sigma_{\bar{X} \bar{X}_{t}} .
$$

Temos, ainda, que 


$$
\begin{aligned}
\operatorname{Var}\{W 32\} & =\operatorname{Var}\left\{\left(\bar{X}_{t .}-\mu_{x}\right)\left(Y_{t}-\mu_{y}\right)-\sigma_{\bar{X} Y}\right\}=\operatorname{Var}\left\{\left(\bar{X}_{t .}-\mu_{x}\right)\left(Y_{t}-\mu_{y}\right)\right\} \\
& \left.=\mathrm{E}\left\{\left[\bar{X}_{t .}-\mu_{x}\right)\left(Y_{t}-\mu_{y}\right)\right]^{2}\right\}-\left\{\mathrm{E}\left[\left(\bar{X}_{t .}-\mu_{x}\right)\left(Y_{t}-\mu_{y}\right)\right]\right\}^{2} \\
& =\mathrm{E}\left\{\left[\left(x_{t}-\mu_{x}\right)+\bar{u}_{t .}\right]^{2}\left[\beta\left(x_{t}-\mu_{x}\right)+e_{t}\right]^{2}\right\}-\sigma_{\bar{X} Y}^{2}
\end{aligned}
$$

Fazendo $\mathrm{E}\left\{\left[\left(x_{t}-\mu_{x}\right)+\bar{u}_{t .}\right]^{2}\left[\beta\left(x_{t}-\mu_{x}\right)+e_{t}\right]^{2}\right\}=S W 32$, temos que

$$
\begin{aligned}
S W 32= & \mathrm{E}\left\{\left[\left(x_{t}-\mu_{x}\right)^{2}+\bar{u}_{t .}^{2}+2\left(x_{t}-\mu_{x}\right) \bar{u}_{t .}\right]\left[\beta\left(x_{t}-\mu_{x}\right)^{2}+e_{t}^{2}+2 \beta\left(x_{t}-\mu_{x}\right) e_{t}\right]\right\} \\
= & \mathrm{E}\left\{\left[\left(x_{t}-\mu_{x}\right)^{2}\right]\left[\beta\left(x_{t}-\mu_{x}\right)^{2}+e_{t}^{2}+2 \beta\left(x_{t}-\mu_{x}\right) e_{t}\right]\right\} \\
& +\mathrm{E}\left\{\left[\left(\bar{u}_{t .}\right)^{2}\right]\left[\beta\left(x_{t}-\mu_{x}\right)^{2}+e_{t}^{2}+2 \beta\left(x_{t}-\mu_{x}\right) e_{t}\right]\right\} \\
& \left.+2 \mathrm{E}\left\{\left[\left(x_{t}-\mu_{x}\right) \bar{u}_{t .}\right)\right]\left[\beta\left(x_{t}-\mu_{x}\right)^{2}+e_{t}^{2}+2 \beta\left(x_{t}-\mu_{x}\right) e_{t}\right]\right\} \\
= & 3 \beta^{2} \sigma_{x x}^{2}+\sigma_{x x} \sigma_{e e}+\beta^{2} \sigma_{x x} \frac{\sigma_{u u}}{r_{t}}+\sigma_{e e} \frac{\sigma_{u u}}{r_{t}} \\
= & 2 \sigma_{\bar{X} Y}^{2}+\sigma_{x x}\left(\beta^{2} \sigma_{x x}+\sigma_{e e}\right)+\frac{\sigma_{u u}}{r_{t}}\left(\beta^{2} \sigma_{x x}+\sigma_{e e}\right) \\
= & 2 \sigma_{\bar{X} Y}^{2}+\sigma_{\bar{X} \bar{X}_{t}} \sigma_{Y Y} .
\end{aligned}
$$

Substituindo (C.142) em (C.141), temos

$$
\operatorname{Var}\{W 32\}=2 \sigma_{\bar{X} Y}^{2}+\sigma_{\bar{X} \bar{X}_{t}} \sigma_{Y Y}-\sigma_{\bar{X} Y}^{2}=\sigma_{\bar{X} Y}^{2}+\sigma_{\bar{X} \bar{X}_{t}} \sigma_{Y Y} .
$$

Temos, também, que

$$
\begin{aligned}
\operatorname{Cov}\{W 31, W 33\} & =E\left[W_{31}-E\left(W_{31}\right)\right]\left[W_{33}-E\left(W_{33}\right)\right]=E\left(W_{31} W_{33}\right) \\
& =E\left\{\left[\left(\bar{X}_{t .}-\mu_{x}\right)^{2}-\sigma_{\bar{X} \bar{X}_{t}}\right]\left[R_{3 t}-\left(r_{t}-1\right) \sigma_{u u}\right] \hat{\lambda}\right\} \\
& =\hat{\lambda} E\left[\left(\bar{X}_{t .}-\mu_{x}\right)^{2}-\sigma_{\bar{X} \bar{X}_{t}}\right] E\left[R_{3 t}-\left(r_{t}-1\right) \sigma_{u u}\right] \\
& =0,
\end{aligned}
$$

$$
\begin{aligned}
\operatorname{Cov}\{W 32, W 33\} & =E\left(W_{32} W_{33}\right) \\
& =E\left\{\left[\left(\bar{X}_{t .}-\mu_{x}\right)\left(Y_{t}-\mu_{y}\right)-\sigma_{\bar{X} Y}\right]\left[R_{3 t}-\left(r_{t}-1\right) \sigma_{u u}\right] \hat{\lambda}\right\} \\
& =\left\{E\left[\left(\bar{X}_{t .}-\mu_{x}\right)\left(Y_{t}-\mu_{y}\right)-\sigma_{\bar{X} Y}\right]\right\} E\left\{\left[R_{3 t}-\left(r_{t}-1\right) \sigma_{u u}\right] \hat{\lambda}\right\} \\
& =0
\end{aligned}
$$


e

$$
\operatorname{Var}\{W 33\}=(\hat{\lambda})^{2} \operatorname{Var}\left\{R_{3 t}-\left(r_{t}-1\right) \sigma_{u u}\right\}=(\hat{\lambda})^{2} \operatorname{Var}\left\{R_{3 t}\right\}=2(\hat{\lambda})^{2}\left(r_{t}-1\right) \sigma_{u u}^{2}
$$

Finalmente, obtemos

$$
\operatorname{Cov}\left\{W_{31}, W_{32}, W_{33}\right\}=\left(\begin{array}{ccc}
2 \sigma_{\bar{X} \bar{X}_{t}}^{2} & 2 \sigma_{\bar{X} Y} \sigma_{\bar{X} \bar{X}_{t}} & 0 \\
2 \sigma_{\bar{X} Y} \sigma_{\bar{X} \bar{X}_{t}} & \sigma_{\bar{X} Y} \sigma_{\bar{X} \bar{X}_{t}}+\sigma_{\bar{X} Y}^{2} & 0 \\
0 & 0 & 2(\hat{\lambda})^{2}\left(r_{t}-1\right) \sigma_{u u}^{2}
\end{array}\right)
$$

onde

$$
\begin{aligned}
& \sigma_{\bar{X} Y}=\beta \sigma_{x x}, \quad \sigma_{\bar{X} \bar{X}_{t}}=\sigma_{x x}+\frac{\sigma_{u u}}{r_{t}}, \quad \sigma_{Y Y}=\beta^{2} \sigma_{x x}+\sigma_{e e} \\
& \text { e } \nu_{n}=\frac{\sum_{t=1}^{n} \frac{1}{r_{t}}}{\sum_{t=1}^{n}\left(r_{t}-1\right)} .
\end{aligned}
$$

De (C.147), temos

$$
\begin{aligned}
\operatorname{Var}\left\{U_{3 t}\right\} & =2 a_{1}^{2} \sigma_{\bar{X} \bar{X}_{t}}+a_{2}^{2} \sigma_{\bar{X} \bar{Y}}^{2}+4 a_{1} a_{2} \sigma_{\bar{X} \bar{Y}} \sigma_{\bar{X} \bar{X}_{t}}+a_{2}^{2} \sigma_{Y Y} \sigma_{\bar{X} \bar{X}_{t}}+2 a_{3}^{2}\left(r_{t}-1\right) \nu_{n}^{2} \sigma_{u u}^{2} \\
& =\left(a_{1}, a_{2}, a_{3}\right) \operatorname{Cov}\left\{W_{31}, W_{32}, W_{33}\right\}\left(a_{1}, a_{2}, a_{3}\right)^{\prime} .
\end{aligned}
$$

De (C.148) e (C.147), podemos escrever

$$
\begin{aligned}
& \left(n^{-1}\right) \sum_{t=1}^{n} \operatorname{Cov}\left\{W_{31}, W_{32}, W_{33}\right\}= \\
& \left(\begin{array}{ccc}
2 \sigma_{x x}^{2}+2\left(\frac{\sum_{t=1}^{n} \frac{1}{r_{t}^{2}}}{n}\right) \sigma_{u u}^{2}+4\left(\frac{\sum_{t=1}^{n} \frac{1}{r_{t}}}{n}\right) \sigma_{x x} \sigma_{u u} & 2 \sigma_{\tilde{X} Y}\left[\sigma_{x x}+\left(\frac{\sum_{t=1}^{n} \frac{1}{r_{t}}}{n}\right) \sigma_{u u}\right] & 0 \\
2 \sigma_{X Y Y}\left[\sigma_{x x}+\left(\frac{\sum_{t=1}^{n} \frac{1}{r_{t}}}{n}\right) \sigma_{u u}\right] & \sigma_{Y Y}\left[\sigma_{x x}+\left(\frac{\sum_{t=1}^{n} \frac{1}{r_{t}}}{n}\right) \sigma_{u u}\right]+\sigma_{X Y}^{2} & 0 \\
0 & 0 & 2 \sigma_{u u}^{2}\left[\frac{\left(\sum_{t=1}^{n} \frac{1}{r_{t}}\right)^{n}\left(r_{t}-1\right)^{2}}{\sum_{t=1}}\right]
\end{array}\right)
\end{aligned}
$$


Supondo $1 \leq r_{t} \leq r<\infty$, temos que

$$
\begin{array}{r}
\kappa=\lim _{n \rightarrow \infty}\left(\frac{\sum_{t=1}^{n} \frac{1}{r_{t}}}{n}\right)<1, \\
\nu_{6}=\lim _{n \rightarrow \infty}\left(\frac{\sum_{t=1}^{n} \frac{1}{r_{t}^{2}}}{n}\right)<1, \\
\nu=\lim _{n \rightarrow \infty}\left(\frac{\sum_{t=1}^{n} \frac{1}{r_{t}}}{\sum_{t=1}^{n}\left(r_{t}-1\right)}\right)<\infty .
\end{array}
$$

Analogamente, para

$$
\begin{aligned}
& U_{2 t}=a_{1}\left[\left(\bar{X}_{t .}-\mu_{x}\right)^{2}-\sigma_{X X_{t}}\right]+a_{2}\left[\left(\bar{X}_{t .}-\mu_{x}\right)\left(Y_{t}-\mu_{y}\right)-\sigma_{\bar{X} Y}\right] \\
& +a_{3} \nu_{4 n}\left[R_{1 t}-\sigma_{u u} I_{r_{t}}(1)\right]
\end{aligned}
$$

obtemos

$$
\begin{aligned}
\operatorname{Var}\left(U_{2 t}\right)=E\left(U_{2 t}^{2}\right)= & 2 a_{1}^{2} \sigma_{\bar{X} \bar{X}_{t}}^{2}+4 a_{1} a_{2} \sigma_{\bar{X} \bar{X}_{t}} \sigma_{\bar{X} Y}+a_{2}^{2}\left(\sigma_{\bar{X} \bar{X}_{t}} \sigma_{Y Y}+\sigma_{\bar{X} Y}^{2}\right) \\
& +a_{3}^{2} \nu_{4 n}^{2}\left(\frac{2 \sigma_{u u}^{2} I_{r_{t}}(1)}{r_{t}-I_{r_{t}}(1)}\right)=\mathbf{a}^{\prime} \Gamma_{2 t} \mathbf{a}<\infty
\end{aligned}
$$

onde $\quad \nu_{4 n}=\frac{\sum_{t=1}^{n} \frac{1}{r_{t}}}{\sum_{t=1}^{n} I_{r_{t}}(1)}, \mathrm{e}$

$$
\begin{aligned}
\Gamma_{2 t} & =\operatorname{Cov}\left\{W_{21 t}, W_{22 t}, W_{23 t}\right\}, \text { com } \\
W_{21 t} & =\left[\left(\bar{X}_{t .}-\mu_{x}\right)^{2}-\sigma_{X X_{t}}\right] \\
W_{22 t} & =\left[\left(\bar{X}_{t .}-\mu_{x}\right)\left(Y_{t}-\mu_{y}\right)-\sigma_{\bar{X} Y}\right] \\
W_{23 t} & =\nu_{4 n}\left[R_{1 t}-\sigma_{u u} I_{r_{t}}(1)\right]
\end{aligned}
$$

e de maneira análoga ao caso anterior, obtemos

$$
\Gamma_{2 \mathrm{t}}=\left[\begin{array}{ccc}
2 \sigma_{X X_{t}}^{2} & 2 \sigma_{X X_{t}} \sigma_{\bar{X} Y} & 0 \\
2 \sigma_{X X_{t}} \sigma_{\bar{X} Y} & \sigma_{X X_{t}} \sigma_{Y Y}+\sigma_{\bar{X} Y}^{2} & 0 \\
0 & 0 & 2 \sigma_{u u}^{2} \nu_{4 n}^{2}\left(\frac{I_{r_{t}}(1)}{r_{t}-I_{r_{t}}(1)}\right)
\end{array}\right]
$$


E ainda, similarmente, para a variável

$U_{1 t}=a_{1}\left[\left(X_{t 1}-\mu_{x}\right)^{2}-\sigma_{X X}\right]+a_{2}\left[\left(X_{t 1}-\mu_{x}\right)\left(Y_{t}-\mu_{y}\right)-\sigma_{X Y}\right]+a_{3} \nu_{1 n}\left(R_{1 t}-I_{r_{t}}(1) \sigma_{u u}\right)$, $\operatorname{com} \nu_{1 n}=n^{-1} \sum_{t=1}^{n} I_{r_{t}}(1)$ para $t=1, \ldots, n$, obtemos

$$
\begin{aligned}
\operatorname{Var}\left(U_{1 t}\right) & =2 a_{1}^{2} \sigma_{X X}^{2}+4 a_{1} a_{2} \sigma_{X X} \sigma_{X Y}+a_{1} a_{3} \nu_{1 n}\left(\frac{4 I_{r_{t}}(1)}{r_{t}}\right) \sigma_{u u}^{2} \\
& +\left[a_{2}^{2}\left(\sigma_{X X} \sigma_{Y Y}+\sigma_{X Y}^{2}\right)\right]+2 a_{3}^{2} \nu_{1 n}^{2}\left(\frac{I_{r_{t}}(1) \sigma_{u u}^{2}}{r_{t}-I_{r_{t}}(1)}\right)=\mathbf{a}^{\prime} \Gamma_{1 t} \mathbf{a}
\end{aligned}
$$

onde $\sigma_{X X}=\sigma_{x x}+\sigma_{u u}, \sigma_{X Y}=\beta \sigma_{x x}, \sigma_{Y Y}=\beta^{2} \sigma_{x x}+\sigma_{e e}$

$$
\begin{aligned}
\Gamma_{1 t} & =\operatorname{Cov}\left\{W_{21 t}, W_{22 t}, W_{23 t}\right\}, \text { com } \\
W_{1 t} & =\left[\left(X_{t}-\mu_{x}\right)^{2}-\sigma_{X X}\right], \\
W_{12 t} & =\left[\left(X_{t}-\mu_{x}\right)\left(Y_{t}-\mu_{y}\right)-\sigma_{X Y}\right], \\
W_{13 t} & =\nu_{1 n}\left[R_{1 t}-\sigma_{u u} I_{r_{t}}(1)\right] .
\end{aligned}
$$

E, novamente, como nos casos anteriores, obtemos

$$
\Gamma_{1 t}=\left[\begin{array}{ccc}
2 \sigma_{X X}^{2} & 2 \sigma_{X X} \sigma_{X Y} & \nu_{1 n}\left(\frac{2 I_{r_{t}}(1)}{r_{t}}\right) \sigma_{u u}^{2} \\
2 \sigma_{X X} \sigma_{X Y} & \sigma_{X X} \sigma_{X Y}+\sigma_{X Y}^{2} & 0 \\
\nu_{1 n}\left(\frac{2 I_{r_{t}}(1)}{r_{t}}\right) \sigma_{u u}^{2} & 0 & \nu_{1 n}^{2}\left(\frac{2 I_{r_{t}}(1)}{r_{t}-I_{r_{t}}(1)}\right) \sigma_{u u}^{2}
\end{array}\right] .
$$




\section{Apêndice D}

\section{Condições suficientes para que a matriz $E\left[\mathbf{A}_{n}\left(\boldsymbol{\theta}^{(1)}\right) \mid \boldsymbol{\theta}^{(0)}, x_{t}^{0}\right]$ seja negativa definida}

Estas condições suficientes foram utilizadas no Capítulo 4 para provarmos o Teorema 4.1. Lembrando que $\theta^{(0)}=\left(\alpha^{0}, \beta^{0}, \sigma_{u u}^{0}, \sigma_{e e}^{0}\right)$ é o verdadeiro valor do vetor dos parâmetros estruturais e $x_{t}^{0}$ é o verdadeiro valor do parâmetro incidental, temos que (veja Cap. 4, eq.4.99)

$$
\begin{aligned}
& E\left[A_{n}\left(\theta^{(1)}\right) \mid \theta^{(0)}, x_{t}^{0}\right]= \\
& =\left(\frac{w}{n \sigma_{u u}^{0}}\right)\left[\begin{array}{cccc}
-\sum_{t=1}^{n} v_{t} & -\sum_{t=1}^{n} v_{t} x_{t}^{0} & 0 & 0 \\
-\sum_{t=1}^{n} v_{t} x_{t}^{0} & -\sum_{t=1}^{n} v_{t} x_{t}^{0^{2}} & \lambda^{0} w \beta^{0} \Sigma_{r s} & -\beta^{0} w \Sigma_{r s} \\
0 & -\lambda \beta^{0} w \Sigma_{r s} & \frac{w^{2}}{\sigma_{u u}^{0}}\left(n c-\beta^{04} \Sigma_{s s}\right) & \frac{-\beta^{02} w^{2}}{\sigma_{u u}} \Sigma_{r s} \\
0 & -\beta^{0} w \Sigma_{r s} & \frac{-w^{2} \beta^{02}}{\sigma_{u u}} \Sigma_{r s} & \frac{w^{2}}{\lambda^{2} \sigma_{u u}^{0}}\left(n b-\lambda^{2} \Sigma_{r r}\right)
\end{array}\right],
\end{aligned}
$$

sendo 


$$
\begin{aligned}
v_{t} & =\frac{r_{t} s_{t}}{\Delta_{t}} ; \quad \Delta_{t}=r_{t} \lambda+s_{t} \beta^{0^{2}} ; \quad \Sigma_{r s}=\sum_{t=1}^{n} \frac{r_{t} s_{t}}{\Delta_{t}^{2}} ; \quad \Sigma_{r r}=\sum_{t=1}^{n} \frac{r_{t}^{2}}{\Delta_{t}^{2}} ; \\
\Sigma_{s s} & =\sum_{t=1}^{n} \frac{s_{t}^{2}}{\Delta_{t}^{2}} ; \quad \bar{\Sigma}_{r s}=\frac{\Sigma_{r s}}{n} ; \quad c=\frac{\bar{r}}{2 w}-\bar{r}+1 ; \quad b=\frac{\bar{s}}{2 w}-\bar{s}+1 ; \\
w & =\frac{\bar{r}+\bar{s}}{\bar{r}+\bar{s}-1} .
\end{aligned}
$$

A seguir, apresentamos o teorema e o corolário utilizados para estabelecer as condições para que a matriz (D.1) seja negativa definida. O teorema pode ser encontrado em muitos livros de algebra linear, como por exemplo, em Lima (1996).

Teorema D.1 A fim de que uma matriz simétrica $\mathbf{A}=\left[a_{i j}\right] \in$ $M(n \times n)$ seja positiva definida é necessário e suficiente que seus menores principais sejam todos positivos.

A prova deste Teorema pode ser encontrada em, por exemplo, Lima (1996, pg 273); bem como a definição de menores principais.

Corolário D.1 A fim de que uma matriz simétrica $A=\left[a_{i j}\right] \in M(n \times n)$ seja negativa definida é necessário e suficiente que seus menores principais de ordem impar sejam negativos e os menores principais de ordem par sejam positivos.

Prova: A é negativa definida se $\mathrm{x}^{\prime} \mathrm{A} \mathbf{x}<0, \forall \mathbf{x} \neq 0 \Rightarrow \mathrm{x}^{\prime}(-\mathrm{A}) \mathrm{x}>0$. Portanto - $\mathrm{A}$ é positiva definida. Seja $\mathrm{A}_{r}=\left(a_{i j}\right), \quad 1 \leq i, j \leq r$, com $1 \leq r \leq n$. Pelo teorema D. 1 o $\operatorname{det}\left(-\mathbf{A}_{r}\right)>0, \forall r \Rightarrow(-1)^{r} \operatorname{det}\left(\mathbf{A}_{r}\right)>0 \Rightarrow$

$$
\operatorname{det}\left(A_{r}\right)=\left\{\begin{array}{cl}
<0 & \text { se r for impar } \\
>0 & \text { se } r \text { for par. }
\end{array}\right.
$$

Condições Suficientes para que a matriz $E\left[\mathrm{~A}_{n}\left(\theta^{(1)}\right) \mid \theta^{(0)}, x_{t}^{0}\right]$ seja negativa definida 
Tendo em vista o Corolário D.1, temos que:

- O menor principal de ordem 1 , determinante de $\mathbf{E}_{1}=\left[-\sum_{t=1}^{n} v_{t}\right]$, é negativo.

- O menor principal de ordem 2 é positivo pois o determinante de $\mathrm{E}_{2}$ é positivo; com efeito:

$$
\begin{aligned}
\operatorname{det}\left(\mathrm{E}_{2}\right) & =\operatorname{det}\left[\begin{array}{cc}
-\sum_{t=1}^{n} v_{t} & -\sum_{t=1}^{n} v_{t} x_{t}^{0} \\
-\sum_{t=1}^{n} v_{t} x_{t}^{0} & -\sum_{t=1}^{n} v_{t} x_{t}^{0^{2}}
\end{array}\right] \\
& =\left(\sum_{t=1}^{n} v_{t}\right)^{2} \times \frac{\sum_{t=1}^{n} v_{t}\left(x_{t}-\bar{x}_{v}\right)^{2}}{\left(\sum_{t=1}^{n} v_{t}\right)} \\
& =\left(\sum_{t=1}^{n} v_{t}\right)^{2} S_{x x}^{(v)}>0,
\end{aligned}
$$

sendo

$$
\bar{x}_{v}=\frac{\sum_{t=1}^{n} v_{t} x_{t}^{0}}{\sum_{t=1}^{n} v_{t}}, \quad S_{x x}^{(v)} \frac{\sum_{t=1}^{n} v_{t}\left(x_{t}^{0}-\bar{x}_{v}\right)^{2}}{\left(\sum_{t=1}^{n} v_{t}\right)}>0, \quad v_{t}=\frac{r_{t} s_{t}}{\lambda r_{t}+\beta^{02} s_{t}}>0 .
$$

O menor principal de ordem $1, \operatorname{det} \mathrm{E}_{1}$, é negativo, e o menor principal de ordem 2, $\operatorname{det} \mathbf{E}_{2}$, é positivo. Portanto satisfazem o Corolário D.1, sem necessidade de se impor condições. No entanto para que o menor principal de ordem 3 seja negativo impomos algumas condições, que apresentamos a seguir.

\section{D.0.1 Condições suficientes para que a matriz $\mathbf{E}_{3}$ tenha determinante negativo}

$$
\begin{aligned}
\operatorname{det}\left(\mathbf{E}_{3}\right)= & \operatorname{det}\left[\begin{array}{ccc}
-\sum_{t=1}^{n} v_{t} & -\sum_{t=1}^{n} v_{t} x_{t}^{0} & 0 \\
-\sum_{t=1}^{n} v_{t} x_{t}^{0} & -\sum_{t=1}^{n} v_{t} x_{t}^{0^{2}} & \lambda w \beta^{0} \Sigma_{r s} \\
0 & \lambda w \beta^{0} \Sigma_{r s} & \frac{w^{2}}{\sigma_{u u}}\left[n c-\beta^{04} \Sigma_{s s}\right]
\end{array}\right] \\
= & \left(-\sum_{t=1}^{n} v_{t}\right)\left(-\sum_{t=1}^{n} v_{t} x_{t}^{02}\right) e_{33}-\left(-\sum_{t=1}^{n} v_{t}\right)\left(\lambda \beta^{0} w \Sigma_{r s}\right)^{2} \\
& -\left(-\sum_{t=1}^{n} v_{t} x_{t}^{0}\right)^{2} e_{33}=
\end{aligned}
$$




$$
=\left(\sum_{t=1}^{n} v_{t}\right)\left[e_{33}\left(\sum_{t=1}^{n} v_{t}\right) S_{x x}^{(v)}+\lambda^{2} \beta^{2} w^{2} \Sigma_{r s}^{2}\right] .
$$

De (D.4), para o $\operatorname{det}\left(\mathrm{E}_{3}\right)$ ser negativo é necessário que $e_{33}<0$, ou seja, $n\left\{\frac{(\bar{r}+\bar{s})(1-\bar{r})+\bar{s}}{2(\bar{r}+\bar{s})}\right\}-$ $\left(\beta^{0}\right)^{4} \Sigma_{r s}<0$, do que decorre que

$$
\bar{r}>1+\left(\frac{\bar{s}}{\bar{r}+\bar{s}}\right)-2\left(\beta^{0}\right)^{4} \bar{\Sigma}_{r s}
$$

Dada a condição (D.5), o $\operatorname{det}\left(\mathbf{E}_{3}\right)$ será negativo se

$$
\begin{gathered}
e_{33}\left(\sum_{t=1}^{n} v_{t}\right) S_{x x}^{(v)}+\lambda^{2} \beta^{2} w^{2} \bar{\Sigma}_{r s}^{2}<0 \text {, ou equivalentemente, se } \\
S_{x x}>S_{11}=\frac{\left(\lambda \beta \bar{\Sigma}_{r s}\right)^{2} \sigma_{u u}}{\bar{u}\left(\beta^{04} \bar{\Sigma}_{s s}-c\right)} .
\end{gathered}
$$

Vamos estabelecer, agora, as condições para que o menor principal de ordem quatro seja positivo, ou seja, que o determinante de $\mathbf{E}_{4}$ seja positivo, em que

$$
E_{n}\left[\mathbf{A}_{n}\left(\boldsymbol{\theta}^{(1)}\right) \mid \boldsymbol{\theta}^{0}, x_{t}^{0}\right]=\left(\frac{w}{n \sigma_{u u}^{0}}\right) \mathbf{E}_{4} .
$$

\section{D.0.2 Condições suficientes para que a matriz $\mathbf{E}_{4}$ tenha determinante positivo}

Temos que

$$
\begin{aligned}
\operatorname{det}\left(\mathbf{E}_{4}\right)= & (-1)^{4+2}\left(-\beta^{0} w \Sigma_{r s}\right) \operatorname{det}\left(\mathbf{E}_{3}^{*}\right)+(-1)^{4+3} \times\left(\frac{-w^{2} \beta^{02} \Sigma_{r s}}{\sigma_{u u}}\right) \operatorname{det}\left(\mathbf{E}_{3}^{* *}\right) \\
& +(-1)^{4+4}\left(e_{44}\right) \operatorname{det}\left(\mathbf{E}_{3}\right)
\end{aligned}
$$

sendo

$$
\begin{aligned}
\operatorname{det}\left(\mathbf{E}_{3}^{*}\right) & =\operatorname{det}\left[\begin{array}{ccc}
-\sum_{t=1}^{n} v_{t} & 0 & 0 \\
-\sum_{t=1}^{n} v_{t} x_{t}^{0} & \lambda \beta^{0} w \Sigma_{r s} & -\beta^{0} w \Sigma_{r s} \\
0 & \frac{w^{2}}{\sigma_{u u}}\left[n c-\beta^{04} \Sigma_{s s}\right] & \frac{-\beta^{02} w^{2}}{\sigma_{u u}} \Sigma_{r s}
\end{array}\right] \\
& =\left(\frac{n^{3} w^{3} \beta^{0} \bar{v} \bar{\Sigma}_{r s}}{\sigma_{u u}}\right) \times\left[\beta^{02}\left(\lambda \bar{\Sigma}_{r s}+\beta^{02} \bar{\Sigma}_{s s}\right)-c\right]
\end{aligned}
$$




$$
\begin{aligned}
\operatorname{det}\left(\mathbf{E}_{3}^{* *}\right) & =\operatorname{det}\left[\begin{array}{ccc}
-\sum_{t=1}^{n} v_{t} & -\sum_{t=1}^{n} v_{t} x_{t}^{0} & 0 \\
-\sum_{t=1}^{n} v_{t} x_{t}^{0} & -\sum_{t=1}^{n} v_{t} x_{t}^{02} & -\beta^{0} w \Sigma_{r s} \\
0 & \lambda \beta^{0} w \Sigma_{r s} & \frac{-\beta^{02} w^{2}}{\sigma_{u u}} \Sigma_{r s} \\
&
\end{array}\right] \\
& =-\left(\frac{n^{3} w^{2} \beta^{02} \bar{v} \bar{\Sigma}_{r s}}{\sigma_{u u}}\right) \times\left(\bar{v} S_{x x}^{(v)}+\lambda \sigma_{u u} \bar{\Sigma}_{r s}\right)
\end{aligned}
$$

e

$$
\begin{aligned}
\operatorname{det}\left(\mathbf{E}_{3}\right) & =\operatorname{det}\left[\begin{array}{ccc}
-\sum_{t=1}^{n} v_{t} & -\sum_{t=1}^{n} v_{t} x_{t}^{0} & 0 \\
-\sum_{t=1}^{n} v_{t} x_{t}^{0} & -\sum_{t=1}^{n} v_{t} x_{t}^{02} & \lambda w \beta^{0} \Sigma_{r s} \\
0 & \lambda w \beta^{0} \Sigma_{r s} & \frac{w^{2}}{\sigma_{u u}}\left[n c-\beta^{04} \Sigma_{s s}\right]
\end{array}\right] \\
& =\left(\frac{n^{3} \bar{v} w^{2}}{\sigma_{u u}}\right)\left[\left(c-\beta^{04} \bar{\Sigma}_{s s}\right) \bar{v} S_{x x}^{(v)}+\left(\lambda \beta^{0} \Sigma_{r s}\right)^{2} \sigma_{u u}\right] .
\end{aligned}
$$

Substituindo (D.9), (D.10) e (D.11) em (D.8), temos

$$
\begin{aligned}
& \operatorname{det} \mathrm{E}_{4}=\left(\frac{n^{4} \bar{v} w^{4}}{\lambda^{2} \sigma_{u u}^{2}}\right) \times \mathrm{E}_{44}, \text { onde } \\
& \qquad \begin{aligned}
E_{44}= & -\left(\lambda \beta^{0} \Sigma_{r s}\right)^{2} \sigma_{u u}^{0}\left[\beta^{02}\left(\lambda \Sigma_{r s}+\beta^{02} \Sigma_{s s}\right)-c\right] \\
& -\left(\beta^{04} \lambda^{2} \Sigma_{r s}^{2}\right)\left(\bar{v} S_{x x}^{(v)}+\lambda \sigma_{u u}^{0} \bar{\Sigma}_{r s}\right)+\left(b-\lambda^{2} \bar{\Sigma}_{r r}\right)\left(\lambda^{2} \beta^{2} \sigma_{u u} \bar{\Sigma}_{r s}^{2}\right) \\
& +\left(b-\lambda^{2} \bar{\Sigma}_{r r}\right)\left(c-\beta^{04} \bar{\Sigma}_{s s}\right)\left(\bar{v} S_{x x}^{(v)}\right) .
\end{aligned}
\end{aligned}
$$

Como $\left(\frac{n^{4} \bar{v} w^{4}}{\lambda^{2} \sigma_{u u}^{2}}\right)>0$, as condições para que $\operatorname{odet}\left(\mathbf{E}_{4}\right)>0$ são estabelecidas ao impor que a expressão $E_{44}$ em (D.12) seja positiva, isto é,

$$
\begin{aligned}
E_{44} & =\left[\left(b-\lambda^{2} \bar{\Sigma}_{r r}\right) c-b \beta^{04} \bar{\Sigma}_{s s}+\lambda^{2} \beta^{04}\left(\bar{\Sigma}_{r r} \bar{\Sigma}_{s s}-\bar{\Sigma}_{r s}^{2}\right)\right] \cdot \bar{v} S_{x x}^{(v)} \\
& -\beta^{04} \lambda^{3} \sigma_{u u} \bar{\Sigma}_{r s}^{3}+\left(b-\lambda^{2} \bar{\Sigma}_{r r}\right)\left(\lambda^{2} \beta^{02} \sigma_{u u} \bar{\Sigma}_{r s}^{2}\right) \\
& -\left(\lambda^{2} \beta^{02} \sigma_{u u}^{0} \bar{\Sigma}_{r s}^{2}\right)\left[\beta^{02}\left(\lambda \bar{\Sigma}_{r s}+\beta^{02} \bar{\Sigma}_{s s}\right)-c\right]>0
\end{aligned}
$$


onde b e c estão definidos em (D.2).

Se as condições (D.5) e (D.6) forem satisfeitas a condição adicional para que o det $\mathbf{E}_{4}$ seja positivo é dada a seguir:

$$
\begin{aligned}
S_{x x}^{(v)}>S_{22}= & \left\{\lambda^{3} \beta^{04} \sigma_{u u}^{0} \Sigma_{r s}^{3}-\left(b-\lambda^{2} \bar{\Sigma}_{r r}\right)\left(\lambda^{2} \beta^{02} \sigma_{u u}^{0} \bar{\Sigma}_{r s}^{2}\right)\right. \\
& \left.+\left(\lambda \beta^{0} \bar{\Sigma}_{r s}\right)^{2} \sigma_{u u} \times\left[\beta^{02}\left(\lambda \bar{\Sigma}_{r s}+\beta^{02} \bar{\Sigma}_{s s}\right)-c\right]\right\} \\
& \times\left\{\bar{v}\left[\left(b-\lambda^{2} \bar{\Sigma}_{r r}\right) c-b \beta^{04} \bar{\Sigma}_{s s}+\lambda^{2} \beta^{04}\left(\bar{\Sigma}_{r r} \bar{\Sigma}_{s s}-\bar{\Sigma}_{r s}^{2}\right)\right]\right\}^{-1} .
\end{aligned}
$$

Concluímos que as três condições suficientes para que $\operatorname{det} \mathbf{E}_{4}>0$, são

$$
\begin{array}{ll}
\mathrm{CM}-1) & \bar{r}>1+\frac{\bar{s}}{\bar{r}+\bar{s}}-2 \beta^{04} \bar{\Sigma}_{s s} \\
\mathrm{CM}-2) & \bar{s}>1+\frac{\bar{r}}{\bar{r}+\bar{s}}-2 \lambda^{2} \bar{\Sigma}_{r r}
\end{array}
$$

e

$$
\text { CM-3) } \quad S_{x x}^{(v)}>\max \left(S_{11}, S_{22}\right)
$$

A expressão $S_{22}$ pode ser escrita de uma forma mais simplificada como

$$
\begin{aligned}
S_{22} & =\frac{\lambda^{2} \beta^{02} \sigma_{u u}^{0} \bar{\Sigma}_{r s}^{2}\left\{\beta^{02}\left(\lambda \bar{\Sigma}_{r s}+\beta^{02} \bar{\Sigma}_{s s}\right)+\lambda\left(\beta^{02} \bar{\Sigma}_{r s}+\lambda \bar{\Sigma}_{r r}\right)-(b+c)\right\}}{\bar{v}\left[\left(b-\lambda^{2} \Sigma_{r r}\right) c-b \beta^{04} \bar{\Sigma}_{s s}+\lambda^{2} \beta^{04}\left(\bar{\Sigma}_{r r} \bar{\Sigma}_{s s}-\bar{\Sigma}_{r s}^{2}\right)\right]} \\
& =\frac{\left\{\lambda^{2} \beta^{02} \sigma_{u u}^{0} \bar{\Sigma}_{r s}^{2}\left\{\beta^{02}\left(\lambda \bar{\Sigma}_{r s}+\beta^{02} \Sigma_{s s}\right)+\lambda\left(\beta^{02} \Sigma_{r s}+\lambda \bar{\Sigma}_{r r}\right)-(b+c)\right\}\right.}{\bar{v}\left[b c-\left(c \lambda^{2} \bar{\Sigma}_{r r}+b \beta^{04} \bar{\Sigma}_{s s}\right)+\lambda^{2} \beta^{04}\left(\Sigma_{r r} \Sigma_{s s}-\Sigma_{r s}^{2}\right)\right]} .
\end{aligned}
$$

\section{D.0.3 Casos particulares}

Caso 1: $s_{t} / r_{t}=k, \quad \forall t=1,2, \ldots, n$.

Neste caso temos:

$$
\begin{aligned}
\bar{\Sigma}_{r s} & =\frac{k}{\left(\lambda+k \beta^{02}\right)^{2}} ; \quad \bar{\Sigma}_{r r}=\frac{1}{\left(\lambda+k \beta^{02}\right)^{2}} ; \quad \bar{\Sigma}_{s s}=\frac{k^{2}}{\Delta_{k}^{2}} ; \quad \bar{\Sigma}_{s s} \bar{\Sigma}_{r r}=\bar{\Sigma}_{r s}^{2} \\
\bar{v} & =\frac{\sum_{t=1}^{n} v_{t}}{n}=\frac{\bar{s}}{\Delta_{k}} ; \quad \Delta_{k}=\lambda+k \beta^{02} ; \quad b=\frac{2-\bar{s}}{2}-\frac{k}{2(k+1)} \\
\mathrm{e} & \\
c & =\frac{2-\bar{r}}{2}-\frac{1}{2(k+1)}
\end{aligned}
$$


Portanto, as condições suficientes para que $E_{n}\left[A_{n}\left(\theta^{(1)} / \theta^{(0)}, x_{t}^{0}\right)\right]$ seja negativa definida ficam

CM-1) $\bar{r}>2-\left(\frac{1}{k+1}\right)-\frac{2 k^{2} \beta^{04}}{\Delta_{k}^{2}}<2$;

CM-2) $\bar{s}>2-\frac{k}{k+1}-\frac{2 \lambda^{2}}{\Delta_{k}^{2}}<2$;

$\mathrm{e}$

CM-3) Temos: $S_{22}=\frac{\lambda^{2} \beta^{02} \sigma_{u u}^{0} k^{2}}{\Delta_{k}^{4} \frac{\bar{s}}{\Delta k}} \times\left\{\frac{1-(b+c)}{b c-\frac{\left(c \lambda^{2}+b k^{2} \beta^{04}\right)}{\Delta_{k}^{2}}}\right\}=\frac{\lambda^{2} \beta^{02} \sigma_{u u}^{0} k^{2}}{2 \bar{s} \Delta_{k}} \times\left[\frac{\bar{r}+\bar{s}-1}{b c \Delta_{k}^{2}-\left(c \lambda^{2}+b k^{2} \beta^{04}\right)}\right]$ e $S_{11}=\frac{\left(\lambda \beta^{0} \frac{k}{\Delta_{k}^{2}}\right)^{2} \sigma_{u u}^{0}}{\frac{\bar{s}}{\Delta_{k}}\left(\frac{k^{2} \beta^{04}}{\Delta_{k}^{2}}-c\right)}=\frac{\lambda^{2} \beta^{02} \sigma_{u u}^{0} k^{2}}{\bar{s} \Delta_{k}\left(k^{2} \beta^{04}-c \Delta_{k}^{2}\right)} \Rightarrow S_{x x}^{(k)}>\max \left(S_{11}, S_{22}\right)$,

onde

$$
\begin{gathered}
S_{x x}^{(k)}=\frac{\sum_{t=1}^{n} s_{t}\left(x_{t}^{0}-\bar{x}_{k}^{0}\right)^{2}}{\sum_{t=1}^{n} s_{t}}, \quad \bar{x}_{k}^{0}=\frac{\sum_{t=1}^{n} s_{t} x_{t}^{0}}{n}, \\
b=1-\frac{k}{2}\left(\frac{\bar{r}+\bar{s}+1}{k+1}\right)=1-\frac{k}{2}\left(\bar{r}+\frac{1}{k+1}\right) \quad \text { e } \quad c=1-\frac{1}{2}\left(\frac{\bar{r}+\bar{s}+1}{k+1}\right)=1-\frac{1}{2}\left(\bar{r}+\frac{1}{k+1}\right) .
\end{gathered}
$$

Caso 2: $\quad r_{t}=s_{t}=r \geq 2$.

As condições (CM-1) e (CM-2) são sempre satisfeitas.

Por outro lado temos:

$$
S_{22}=\frac{\lambda^{2} \beta^{02} \sigma_{u u}^{0}}{r \Delta}\left(\frac{1-(b+c)}{b\left(c \Delta^{2}-\beta^{04}\right)-c \lambda^{2}}\right) \quad \text { e } \quad S_{11}=\frac{\lambda^{2} \beta^{02} \sigma_{u u}}{r \Delta\left(\beta^{04}-c \Delta^{2}\right)},
$$

onde $\Delta=\lambda+\beta^{02}$ e $b=c=\frac{-2 r+3}{4}$.

Portanto, temos

$$
S_{22}=\frac{\lambda^{2} \beta^{02} \sigma_{u u}^{0}}{r \Delta} \times\left\{\frac{2(2 r-1)}{(-2 r+3)\left[c \Delta^{2}-\left(\lambda^{2}+\beta^{04}\right)\right]}\right\} .
$$

A condição (CM-3) resulta em: $S_{x x}>\max \left\{S_{11}, S_{22}\right\}$; onde: $S_{x x}=\sum_{t=1}^{n} \frac{\left(x_{t}^{0}-\bar{x}^{0}\right)^{2}}{n}$.

Caso 3: $r_{t}=r \geq 2$ e $s_{t}=1$.

Neste caso temos

$$
v_{t}=\frac{r_{t} s_{t}}{\Delta_{t}}=\frac{r}{\left(r \lambda+\beta^{02}\right)}=\frac{r}{\Delta_{r}} ;
$$




$$
\begin{aligned}
& \Delta_{t}=\Delta_{r}=r \lambda+\beta^{02} ; \\
& \sum_{t=1}^{n} \frac{v_{t}}{\Delta_{t}}=\frac{n r}{\Delta_{r}^{2}} ; \quad \sum_{t=1}^{n} \frac{r_{t}^{2}}{\Delta_{t}^{2}}=\frac{n r^{2}}{\Delta_{r}^{2}} ; \\
& \sum_{t=1}^{n} \frac{s_{t}^{2}}{\Delta_{t}^{2}}=\frac{n}{\Delta_{r}^{2}} ; \quad w=\frac{\bar{r}+\bar{s}}{\bar{r}+\bar{s}-1}=\frac{r+1}{r} \quad \mathrm{e} \\
& c=\frac{\bar{r}}{2 w}-\bar{r}+1=\frac{-r^{2}+2}{2(r+1)}<0 ; \quad \text { pois, } \quad r \geq 2 \text { e } \quad b=\frac{r}{2(r+1)}>0 .
\end{aligned}
$$

Substituindo as igualdades acima em (D.13) e (D.13), temos:

(CM-1) $\bar{r}>2-\left(\frac{r}{r+1}\right)-\frac{2 \beta^{04}}{r^{2} \Delta_{r}^{2}}<2$

Esta condição é sempre satisfeita pois $r_{t} \geq 2, \forall t$.

$(\mathrm{CM}-2) \bar{s}=s_{t}=1>2-\frac{1}{r+1}-\frac{2 \lambda^{2} r^{2}}{\Delta_{r}^{2}}$.

Portanto, para que a condição (CM-2) seja satisfeita, devemos ter:

$$
1-\frac{1}{r+1}-\frac{2 r^{2}}{r+\frac{\beta^{02}}{\lambda}}<0 \Rightarrow r>\left(\frac{\beta^{02}}{\lambda}-1\right)+\sqrt{2\left(\frac{\beta^{02}}{\lambda}-\frac{1}{2}\right)^{2}+\frac{1}{2}} .
$$

Para a terceira condição (CM-3) temos:

$$
S_{11}=\frac{r \lambda^{2} \beta^{02} \sigma_{u u}^{0}}{\left(r \lambda+\beta^{02}\right)\left[\beta^{04}-c\left(\lambda+r \beta^{02}\right)^{2}\right]}=\frac{r \lambda^{2} \beta^{02} \sigma_{u u}^{0}}{\Delta_{r}\left(\beta^{04}-c \Delta_{r}^{2}\right)} .
$$

Como $c<0$, segue-se que, $0<S_{11}<\infty$. Assim, para para satisfazer a condição (CM-3) devemos ter:

$S_{x x} \geq \max \left\{S_{11}, S_{22}\right\}$, onde

$$
S_{22}=\frac{\lambda^{2} \beta^{02} \sigma_{u u}^{0} r^{2}}{2 \Delta_{r}\left[b c \Delta_{r}^{2}-\left(c r^{2} \lambda^{2}+b \beta^{04}\right)\right]}=\frac{\lambda^{2} \beta^{02} \sigma_{u u}^{0} r^{2}}{2 \Delta_{r}\left[-c\left(r^{2} \lambda^{2}-b \Delta_{r}^{2}\right)-b \beta^{04}\right]},
$$

com

$$
S_{x x}=\sum_{t=1}^{n} \frac{\left(x_{t}-\bar{x}_{t}\right)^{2}}{n}, \quad 0<b=\frac{r}{2(r+1)}<\frac{1}{2} \quad \text { e } \quad c=\frac{-r^{2}+2}{2(r+1)}<0 .
$$

Conclusão: Pouca variabilidade de $x_{t}$, ou seja, $S_{x x}$ pequeno, pode levar a que $E\left[A_{n}\left(\boldsymbol{\theta}^{(1)}\right) \mid \boldsymbol{\theta}^{(0)}, x_{t}^{0}\right]=$ não seja negativa definida. 


\section{Referências}

Arellano-Valle, R. B. (1994). Distribuições elípticas: propriedades, inferência e aplicações a modelos de regressão. Ph. D. thesis, IME-USP.

Barnett, V. D. (1969). Simultaneous pairwise linear structural relationships. Biometrics, 25, 129-142.

Bickel, P. and K. A. Doksum (1977). Mathematical Statistics. Holden-Day,INC.

Boyles, R. A. (1983). "On the convergence of EM algorithm". J.R.Statistic.Soc., $B, 45,47-50$.

Cabral, C. R. B. (2000). Testes de Distância em Modelos de Regressão com Erros nas Variáveis. Ph. D. thesis, IME-USP.

Chan, L. K. and T. K. Mak (1979a). Maximum likelihood estimation of a line structural relationship with replication. J.R.Statistic.Soc., B, 41, 263-268.

Chan, L. K. and T. K. Mak (1979b). On the maximum likelihood estimation of a linear structural relationship when the intercept is known. Journal of Multivariate Analysis, 9, 304-313.

Cheng, C. and J. Van-Ness (1991). On the unreplicated ultrastructural model. Biometrika, 78, 442-445.

Dempster, A. P., N. M. Laird and D. B. Rubin (1977). " Maximum Likelihood from incomplete data via the EM algorithm". Journal of the Royal Statistical Society, $B, 39,1-38$.

Dolby, G. R. (1976). The ultrastructural relation: a synthesis of the functional and 
structural relations. Biometrika, 63, 39-50.

Dorff, M. and J. Gurland (1960). Estimation of the parameters of a linear functional relation. J. R. Statist. Soc., B, 23, 160-170.

Durret, R. (1991). Probability Theory and Examples. Wadsworth, Brooks \& Cole Publishing Company.

Fleming, W. H. (1966). Functions of Several Variables. Addison-Wesley.

Fuller, W. A. (1987). Measurement Error Models. John Wiley and Sons.

Fuller, W. A. (1995). Estimation in the presence of measurement error. International Statistical Review, 63, 121-147.

Gallo, P. P. (1982). Properties of Estimators in Error-in-Variables Regression Models. $\mathrm{Ph}$. D. thesis, University of North Carolina, Chapel Hill NC, USA.

Gimenez, P. C. (1997). Inferência em modelos com erros nas variáveis através do método do escore corrigido. Ph. D. thesis, IME-USP.

Gimenez, P. C. and H. Bolfarine (1997). Corrected score functions in classical errorin-variables and incidental parameter models. Austral.J.Statist., 39, 325-344.

Gleser, L. J. (1981). Estimation in a multivariate error-in-variables regression model: large samples results. Annals of Statistics, 9, 24-44.

Gleser, L. J. (1985). A note on G.R.Dolby's unreplicated ultraestructural error in variables model. Biometrika, 72, 117-124.

James, B. (1996). Probabilidade: Um Curso Em Nivel Intermediário. (2nd ed.). Projeto Euclides.

Johnson, R. A. and D. W. Wichern (1992). Applied Multivariate Statistical Analysis (3nd ed.). Printice-Hall.

Kendall, M. G. and A. Stuart (1979). The Advanced Theory of Statistics, Cap29 (3rd ed.), Volume 2. New York: Hafner.

Kimura, D. (1992). Functional comparative calibration using the EM algorithm. Biometrics, 48, 1263-1271. 
Lima, E. L. (1996). Algebra Linear. IMPA - Coleção Matemática Universitária.

Lindley, D. and G. M. El-Sayad (1968). The bayesian estimation of linear functional relationship. J.Roy.Statist.Soc., B, 30, 190-202.

Lindley, D. V. (1947). Regression lines and the linear functional relationship. J.Roy.Statist.Soc.Supp., 9, 218-244.

Madansky, A. (1959). The fitting of straight lines when both variables are subject to error. J.Amer.Statist.Assoc. 54, 173-205.

Mak, T. K. (1982). Estimation in the presence of incidental parameters. The Canadian Journal of Statistics, 10, $121-123$.

Miller, J. J. (1977). Asymptotic properties of maximum likelihood estimates in the mixed model of the analysis of variance. The Annals of Statistics, 5, 746-762.

Morgado, A. C. O., J. B. Pitombeira, P. C. P. Carvalho and P. Fernandez (1991). Análise Combinatória e Probabilidade. Sociedade Brasileira de Matemática.

Muirhead, R. J. (1982). Aspects of Multivariate Statistical Theory. John Wiley \& Sons.

Nascimento, J. (1994). Parametrização ortogonal e outras contribuições ao modelo com erros nas variáveis. $\mathrm{Ph}$. D. thesis, IME-USP.

Neyman, J. and E. L. Scott (1948). Consistent estimates based on partially consistent observations. Econometrica, 6, 1-16.

Patefield, W. M. (1977). On the information matrix in the linear functional relationship problem. Appl. Statistic, 26, 69-70.

Patefield, W. M. (1978). The unreplicated ultrastructural relation: Large sample properties. Biometrika, 65, 535-540.

Peres, A. D. Q. (1987). Uma aplicação do algoritmo EM no modelo log-linear. Master's thesis, IMPA.

Rao, C. R. (1973). Linear Statistical Inference and Its Applications (2nd ed.). John Wiley \& Sons. 
Richards, F. S. G. (1961). A method of maximum-likehood estimation. J.R.Statist.Soc., B, 23, 469-473.

Rojas, M. J. G. (1995). Calibração comparativa estrutural e funcional. Ph. D. thesis, IME-USP.

Schafer, D. and G. Purdy (1996). Analysis for errors in variables regression with replicate measurements. Biometrika, 83, 813-824.

Searle, S., G. Casela and C. MacCulloch (1992). Variance Components. Wiley New York.

Sen, P. K. and J. M. Singer (1993). Large sample methods in statistics: an introduction with applications. Chapman \& Hall.

Solari, M. E. (1969). The maximum likelihood solution of the problem of estimating a linear functional relationship. J. Roy. Statist, B, 31, 372-375.

Sprent, P. (1990). Some history of functional and structural relationships. American Mathematical Society, 112, 3-15.

Tanner, M. A. (1996). Tools for Statistical Inference. Methods for the Exploration of Posterior Distributions and Likelihood Functions (Third ed.).

Tukey, J. W. (1951). Components in regression. Biometrics, 7, 33-69.

Vilca-Labra, F. E. (1996). Modelo de regressão elíptico com erros nas variáveis. $\mathrm{Ph}$. D. thesis, IME-USP.

Wu, C. F. (1981). "On the convergence of EM algorithm". Technical Report. Department of Statistics, University of Wisconsin, Madison.

Zellner, A. (1971). An Introduction to Bayesian Inference in Econometrics. Wiley, New York. 